Travaux et documents du CIRAC

\title{
ALLEMAGNE : COMPÉTITIVITÉ ET \\ DYNAMIQUES TERRITORIALES
}

Sous la direction d'Isabelle BOURGEOIS 


\section{Allemagne : compétitivité et dynamiques territoriales}

Isabelle Bourgeois (dir.)

DOI : 10.4000/books.cirac. 679

Éditeur : IFAEE

Année d'édition : 2007

Date de mise en ligne : 13 décembre 2017

Collection : Travaux et documents du CIRAC

ISBN électronique : 9782905518576

\section{QboOKS}

http://books.openedition.org

\section{Édition imprimée}

ISBN : 9782905518354

Nombre de pages : 144

\section{Référence électronique}

BOURGEOIS, Isabelle (dir.). Allemagne : compétitivité et dynamiques territoriales. Nouvelle édition [en ligne]. Cergy-Pontoise : IFAEE, 2007 (généré le 02 octobre 2020). Disponible sur Internet : <http:// books.openedition.org/cirac/679>. ISBN : 9782905518576. DOI : https://doi.org/10.4000/books.cirac. 679.

\section{(C) IFAEE, 2007}

Conditions d'utilisation

http://www.openedition.org/6540 
Travaux et documents du CIRAC

\section{ALLEMAGNE : COMPÉTITIVITÉ ET}

\section{DYNAMIQUES TERRITORIALES}

Sous la direction d'Isabelle BOURGEOIS

\section{CIRAC}






\section{ALLEMAGNE : COMPÉTITIVITÉ ET \\ DYNAMIQUES TERRITORIALES}

Sous la direction d'Isabelle BOURGEOIS

$$
\text { CIRAC }
$$




\section{Travaux et documents du CIRAC}

Collection dirigée par René Lasserre

(C) CIRAC, 2007

CIRAC, c/o Université de Cergy-Pontoise 33 Boulevard du Port - 95011 CERGY-PONTOISE CEDEX ISBN 978-2-905518-35-4 


\title{
ALLEMAGNe : COMPÉTITIVITÉ ET DYNAMIQUES TERRITORIALES
}

\author{
TABLE DES MATIĖRES
}

\section{INTRODUCTION}

\section{Isabelle Bourgeois}

La gouvernance territoriale allemande

à l'épreuve de la globalisation et de l'intégration européenne .................................................... 7

I - PolyCENTRISME ET DIVERSITÉ DES POLITIQUES STRUCTURELLES ..........31

\section{Isabelle Bourgeois}

Compétitivité des territoires : état des lieux et perspectives

Markus Gabel

Aufbau Ost : entre rattrapage et différenciation.

II - PORTRAITS DE LÄNDER.

Isabelle Bourgeois

Rhénanie du Nord-Westphalie : reconversion du charbon au high-tech.

Isabelle Bourgeois

Bavière : la patiente construction d'une économie performante

\section{Isabelle Bourgeois}

Bade-Wurtemberg : une compétitivité à taille humaine.

Michèle Weinachter

Hambourg : région métropole européenne et site portuaire international

Michèle Weinachter

Saxe : renaissance industrielle et excellence high-tech.

Marie-Hélène Pautrat

Brandebourg : la quête d'une nouvelle image.

INDEX DES CARTES, TABLEAUX ET ENCADRÉS 



\title{
La gouvernance territoriale allemande à l'épreuve de la globalisation et de l'intégration européenne
}

\author{
Isabelle Bourgeois
}

\begin{abstract}
L'économie allemande est foncièrement compétitive. Sa faiblesse des quinze dernières années n'était que le signe d'une crise d'adaptation à un contexte en mutation : unité allemande, élargissement de l'Europe à l'est, globalisation. Cette compétitivité est liée à la structure sectorielle de son économie, spécialisée dans les biens d'équipement de moyenne et haute technologie, de même qu'à la place qu'elle a su conquérir dans le nouveau partage mondial du travail. Elle est liée plus encore à l'organisation polycentrique des activités sur son territoire comme des lieux de décision économiques et politiques. L'organisation fédérale des pouvoirs, construite sur une double logique d'autonomie et de solidarité, favorise la concurrence des choix de gouvernance. Cette compétition permanente, qui n'exclut pas la coopération, nourrit un processus de confrontation et d'adaptation des pratiques qui est le véritable ressort des performances allemandes. Elle est le fait des acteurs économiques, mais aussi des Länder constitutifs de la République fédérale et qui sont de véritables Etats. Souverains sur leur territoire, ils mènent chacun sa propre politique de développement structurel régional; ce sont eux qui constituent la force productive de l'Allemagne. "Berlin " n'est pas un acteur économique, la Fédération n'est que l'entité institutionnelle supérieure où se concrétise la cohésion étatique de cet ensemble composé de 16 Etats membres de taille, de culture et de dynamisme très divers. L'organisation du fédéralisme allemand respecte ces particularismes, les pouvoirs se déléguant du bas vers le haut. Si elle a fait ses preuves depuis 1949, elle est mise à mal aujourd'hui par une double évolution : une approche égalitariste a pris le pas sur le principe de concurrence, et l'accélération de l'intégration européenne révèle les limites d'un système de gouvernance à deux niveaux. La question cruciale pour l'avenir de la compétitivité allemande est dès lors celle-ci : quelle peut et doit être la place des Länder dans un mode de gouvernance désormais multi-niveaux, intégrant l'échelon européen?
\end{abstract}

La nouvelle donne géopolitique du modèle allemand

L'Allemagne a renoué, depuis 2006, avec son rôle de 'locomotive' de l'économie européenne, surmontant une longue phase de faiblesse qui se manifestait par une croissance en dents de scie depuis l'Unité (1990). Cette faiblesse avait une triple origine. La première est l'effort consenti à la reconstruction de l'économie est-allemande : bon an mal an, l'ouest transférait $4 \%$ de son PIB aux économies émergentes sur le territoire de l'ex-RDA. Cela avait mené à " une forte hausse de l'endettement public et du poids des prélèvements fiscaux et sociaux inhibant en retour les forces de croissance ", comme l'expliquait le Conseil des Sages (Sachverständigenrat) chargé d'évaluer l'évolution de l'économie et de la politique économique allemande, dans son rapport annuel de 2002 (§ 342). 
La seconde raison, indépendante, tient aux mutations globales de l'économie dont l'accélération se produisait au même moment et plaçait le "modèle rhénan " face à un défi de gouvernance : la réactivité exigée par la globalisation des activités remet en question une structuration institutionnelle complexe des processus de décision - par définition multiacteurs outre-Rhin, qu'il s'agisse des échelons territoriaux, des pouvoirs publics ou de la société civile organisée.

La troisième raison relève des mutations des valeurs. Les valeurs de sécurité et de solidarité/partage au cœur du modèle social développé après le "miracle économique » ont fini, la longue prospérité et la progression des acquis sociaux aidant, par se transmuer en une sorte de 'droit au confort' matériel et au maintien des acquis, aujourd'hui en flagrante contradiction avec l'impératif de concurrence et de compétitivité qu'implique une économie ouverte comme celle de l'Allemagne. Dans son acception originelle, l'émulation des acteurs économiques (et des territoires) est pourtant la condition sine qua non de la compétitivité, ellemême source de la création de ces richesses qu'on peut ensuite redistribuer dans le respect de la solidarité. C'est sur ces valeurs et principes qu'est établi l'ordre économique et social allemand et, plus généralement, l'ordre étatique. "La République fédérale d'Allemagne est un Etat fédéral démocratique et social », stipule l'art. $20 \S 1$ de la Loi fondamentale. Roman Herzog, qui fut vice-président, puis président du Tribunal constitutionnel fédéral (1983-94) avant d'occuper les fonctions de président de la République (1994-99), l'explicite ainsi : "Démocratie veut dire concurrence, Etat social signifie solidarité. Ce qui importe, c'est le mélange des deux, et le dosage » (Herzog, 2006). Or c'est ce « dosage » qui s'est dénaturé au fil des décennies.

La lente évolution avait contribué à figer les fondements du « modèle rhénan », transformant la quête du consensus et de l'équilibre des forces - des processus par nature dynamiques - en une exigence de stabilité perpétuelle générant un certain immobilisme. Si cette mutation affecte l'ensemble de la société, elle trouve a fortiori son reflet au niveau structurel et politique, qu'il s'agisse de la praxis des mécanismes de solidarité au cœur du fédéralisme financier ou de la politique de cohésion territoriale. Alors que la compétition internationale implique la différenciation, le principe constitutionnel qui vise à la "réalisation de conditions de vie équivalentes » sur l'ensemble du territoire (art. $72 \S 2$ de la Loi fondamentale) contribue à cimenter les acquis, car le concept d'équité des chances qui le fonde a subi au fil du temps une interprétation le transformant en une sorte de 'droit à l'égalité'. En ce qui concerne la compétitivité des territoires, la conséquence en est un nivellement des performances des Länder qui tasse la compétitivité globale de l'économie allemande. "L’homogénéité n'est pas productive ; c'est la diversité qui est le facteur productif ", rappelle ainsi R. Herzog, dans la continuité d'une série d'arrêts du Tribunal constitutionnel fédéral, dont le principal est celui du 11 novembre 1999, rendu à propos de la péréquation financière entre les Länder (Länderfinanzausgleich) qui est une des expressions concrètes de la solidarité budgétaire appliquée aux collectivités. Dans ce jugement, la Cour avait dû rappeler un autre principe fondateur de l'ordre constitutionnel libéral et démocratique de l'Allemagne, corollaire de l'équité des chances : "l'interdiction du nivellement 》 (Nivellierungsverbot).

A ces trois points, il convient d'en ajouter un quatrième, qui coïncide lui aussi dans le temps : l'accélération du processus d'intégration européenne. Ses effets se situent à plusieurs niveaux. L'Union économique et monétaire a contribué à ordonner le marché intérieur, créant une stabilité et une transparence favorables à l'activité, mais accroissant aussi la 
mobilité des facteurs au sein de l'UE. La progression de l'harmonisation de la réglementation européenne y a contribué elle aussi. Mais dans le même temps, faute d'intégration politique suffisante, et surtout de répartition claire des compétences respectives entre les échelons communautaire, national, régional (et communal), les pouvoirs de « Bruxelles » se sont accrus, rognant progressivement le pouvoir d'initiative des Etats membres de l'UE. L'écrasante majorité des textes votés par les parlements nationaux relève aujourd'hui de l'adoption de «l'acquis communautaire » (mise en conformité du droit national avec les règlements, transcription de directives...).

Outre-Rhin, cette évolution affecte particulièrement les Länder dont le rôle est doublement déterminant pour le cadre réservé aux activités. Etats membres constitutifs de la République fédérale, ils sont d'une part représentés par leur exécutif (entre 3 et 6 ministres par Land, dont le chef de gouvernement de chacun d'entre eux) au Bundesrat. Par cette chambre parlementaire des Länder à l'échelon fédéral, ils co-décident de la politique menée par le gouvernement fédéral. D'autre part, sur leur propre territoire, leurs gouvernements et parlements disposent d'une large latitude d'action politique.

La poursuite de l'intégration européenne a également mené à l'élargissement de l'UE à 27 membres. L'intégration des Etats est-européens fut une 'réunification' économique et politique de l'espace européen, se traduisant par une extension du marché communautaire, mais aussi par la nécessité de repenser la politique de cohésion territoriale au niveau de l'UE. L'Allemagne, autrefois au cœur de la partition entre blocs occidental et soviétique, a été et reste la première concernée par ces évolutions, d'autant qu'elle est directement confrontée, du fait de sa proximité géographique avec les nouveaux Etats membres limitrophes, à l'émergence d'économies attrayantes en termes de niveaux salariaux comme de qualifications, et que l'extension vers l'est de l'espace communautaire crée de nouveaux équilibres et force un nouveau partage du travail.

Enfin, la stratégie de compétitivité que se sont donnés les Etats membres de l'UE lors du sommet de printemps de 2000 à Lisbonne (« Stratégie de Lisbonne ») influe elle aussi sur les choix allemands. Les Etats membres avaient décidé de faire de l'UE, à l'horizon 2010, "l'économie la plus compétitive et la plus dynamique du monde ». L'un des volets de cette stratégie, reconfigurée au printemps 2005, porte sur l'innovation (objectif : $3 \%$ du PIB consacrés à la $R \& D$ ) et plus particulièrement dorénavant sur sa déclinaison à l'échelon régional grâce à la combinaison des politiques d'innovation technologique avec la politique de cohésion territoriale, tant à l'échelon communautaire que national.

Malgré ces mutations imperceptibles, mais profondes, qui affectent chacune des économies européennes, l'Allemagne a su surmonter ses faiblesses en commençant à adapter ses politiques aux nouveaux défis grâce à une prise de conscience précoce des enjeux : en 1993, la crise provoquée par les restructurations d'une économie mondiale en voie de globalisation accélérée succède brutalement au boom qui avait suivi l'Unité. Dans ce contexte émerge un débat sur l'attractivité de l'Allemagne en tant que site de production (Standort Deutschland). Le débat débouche sur la publication en septembre 1993, par le gouvernement fédéral, d'un rapport sur la « Préservation de l'avenir du site Allemagne » (Zukunftssicherung des Standortes Deutschland). Sur la base d'une analyse de l'évolution des tendances globales et de l'identification des handicaps propres à l'Allemagne, il propose une thérapie : "L 'avenir des performances de notre site dépend foncièrement des valeurs et attitudes mentales qui déterminent le climat de la société allemande. Nous ... ne devons pas nous adonner à l'illusion 
que nous pouvons nous reposer sur nos acquis... Le changement nécessaire ne peut pas être le seul fait de l'Etat ou de la sphère politique. La préservation du site Allemagne concerne tout un chacun : le Bund, les Länder et les communes aussi bien que les partis politiques, les partenaires sociaux, les fédérations professionnelles et les Eglises, ou encore les écoles et les institutions culturelles, médias inclus » (p. 5). L'approche allemande est collective, proposant une stratégie conjuguée incluant l'ensemble de la société et, bien sûr, des échelons territoriaux ; elle est par essence multi-niveaux. Mais de ce fait aussi, l'Allemagne s'enlise d'abord dans cette « germanosclérose » qui a tant préoccupé ses partenaires.

Cette situation amène le Conseil des Sages à replacer, dans son rapport annuel de 1997/98, l'impératif des réformes dans leur contexte global : "Alors que l'environnement mondial subit de profondes mutations et dans la perspective de l'impact qu'auront l'union monétaire comme l'élargissement de l'UE à l'est, il importe d'améliorer les conditions permettant que l'initiative privée débouche sur des activités marchandes afin qu'un plus grand nombre de personnes trouve un emploi et afin que soit préservée la prospérité » (introduction). Et il formule des recommandations. Elles reposent toutes sur une approche en termes de «politique de l'offre » (et non de la demande), telle que la développe le Conseil des Sages depuis le milieu des années 1970, considérant que, à l'ère de la globalisation, elle est la seule à même de redynamiser la compétitivité de l'Allemagne.

Une telle politique de l'offre consiste en premier lieu à "créer un environnement fiable et stable " pour l'activité. Cela passe par une "politique économique orientée sur le long terme » (§ 293), et surtout menée en coopération par trois types d'acteurs : l'Etat, avec sa politique budgétaire, la Bundesbank avec sa politique monétaire (aujourd'hui la BCE) et les partenaires sociaux avec «leur autonomie et leur responsabilité en matière de politique salariale ». Le fonctionnement de cette coopération repose sur une logique contractuelle construite sur la confiance mutuelle: ce qui «lui donne vie, c'est que les prestations générées dans un domaine sont honorées par d'autres acteurs et que, en retour, les prestations préalables ne peuvent voir le jour que si ceux qui les génèrent peuvent se reposer en toute confiance sur l'attente de les voir honorées » (\$294). Autrement dit : "la croissance n'est pas réalisée par l'Etat ; elle est au contraire la résultante de ces innombrables efforts humains " (\$295).

Une telle politique est d'autant plus nécessaire dans le nouvel environnement mondial et européen que "les politiques économiques nationales voient leurs effets largement limités par le degré croissant d'interdépendance des relations économiques ». En effet, "la globalisation aggrave les problèmes des pays qui ne parviennent pas à aménager les conditions réservées à l'offre de telle manière que la main d'œuvre disponible soit occupée sur le marché de la production. De mauvaises conditions réservées à l'offre sont le résultat des manquements et négligences domestiques » (§306), rappelait le Conseil.

Ces divers appels, doublés du constat amer et récurrent que l'Allemagne tendait à s'installer dans le rôle de « lanterne rouge de l'Europe », ont fini par porter leurs fruits. Les réformes structurelles visant à réduire le poids des prélèvements (retraites, santé, consolidation budgétaire) sont en cours depuis le lancement, par le chancelier Gerhard Schröder en mars 2003, de l'Agenda 2010. Le gouvernement de grande coalition mené par la chancelière Angela Merkel s'inscrit dans la continuité de cette politique de réformes. Elle se conjugue à la longue politique de modération salariale menée par les partenaires sociaux et à l'intense effort de restructuration menée par les entreprises désireuses de préserver leur performance 
globale sur le marché mondial, créant un cercle vertueux et collectif de rénovation qui commence à libérer la dynamique de croissance de l'économie allemande.

L'Allemagne est ainsi parvenue à surmonter son handicap particulier, l'effort gigantesque consenti à la transformation des économies des cinq Länder est-allemands issus du territoire de l'ex-RDA. Certes, considérés globalement, les nouveaux Länder ne sont pas devenus ces «paysages fleuris » dont rêvait le chancelier Kohl après la chute du Mur, mais leurs économies se sont normalisées : la structure des activités s'y approche aujourd'hui de la moyenne allemande (environ $70 \%$ de services, un peu moins de $30 \%$ d'industrie, et une agriculture résiduelle), même si le tissu industriel reste sous-développé par endroits, comme dans ce Mecklembourg-Poméranie où s'est déroulé, en juin 2007, le Sommet du G8.

Elles se sont normalisées surtout en ce sens qu'elles présentent des disparités territoriales aujourd'hui ni plus ni moins prononcées qu'à l'ouest : si le Brandebourg qui entoure la villeEtat de Berlin se caractérise par une faible dynamique, sauf dans la ceinture de la capitale où se concentrent les activités high-tech, la Saxe de son côté est redevenue un pôle industriel performant voué à l'automobile (axe Zwickau-Dresde), bien que ses marges septentrionales (limitrophes du Brandebourg et de la Saxe-Anhalt) et orientales (Pologne) se caractérisent par un retard économique certain. Leur situation ne diffère plus guère de celle des Länder de l'ouest qui présentent des disparités comparables, avec les anciennes régions industrielles en restructuration comme le bassin de la Ruhr (Rhénanie du Nord-Westphalie) ou celles qui avaient été coupées de leurs zones d'échanges est-européennes par le Rideau de Fer comme tout le flanc oriental de la Bavière. Ou encore toutes celles qui se trouvent éloignées des zones à forte concentration d'activité, établies aux croisements des principaux axes de communication est-ouest et nord-sud au sein de l'espace allemand et européen.

Concentration des activités dans l'espace européen :

l'Allemagne est au cœur de la « banane bleue »

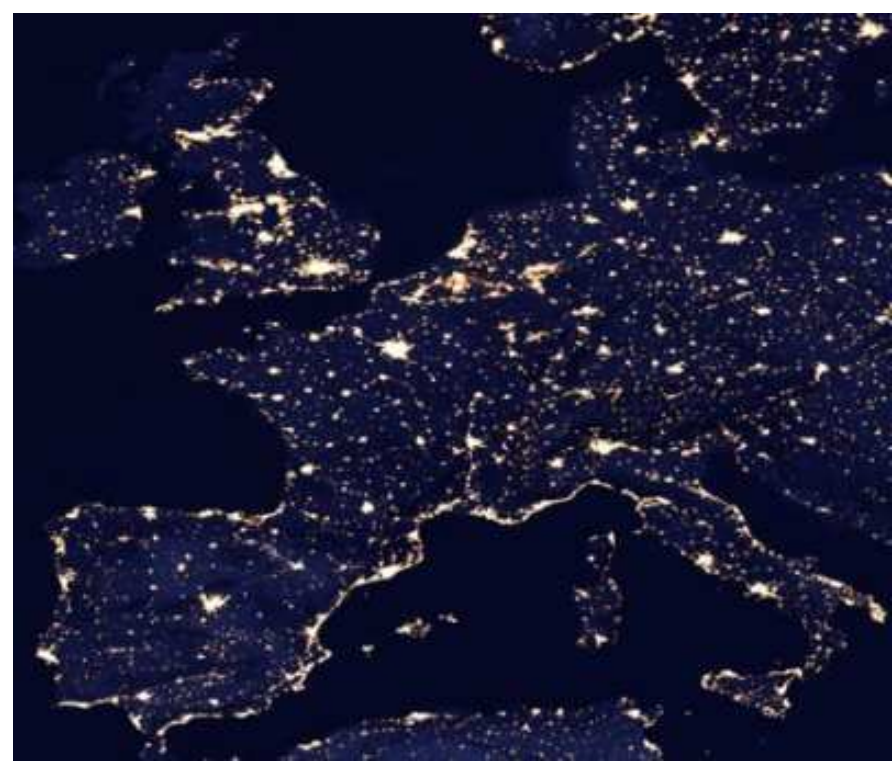

Source : Deutsches Luft- und Raumfahrtzentrum (DLR). 
Il n'y a plus réellement de clivage est-ouest outre-Rhin (sauf en ce qui concerne la démographie et les flux migratoires qui pénalisent les Länder de l'est). Les nouveaux Länder se sont insérés économiquement dans ce clivage nord-sud du dynamisme économique et de la compétitivité si caractéristique de l'Allemagne placée au cœur de cette «banane bleue », chaîne des pôles de concentration d'activités européenne qui s'étend de la Grande-Bretagne au nord de l'Italie en passant par le Benelux, longeant l'axe rhénan et traversant la Bavière (région de Munich) et qui est dénommée ainsi dans la mesure où les images satellites nocturnes révèlent ces pôles grâce à leur intensité lumineuse.

\section{Une économie construite sur les réseaux et les échanges}

En réalité, si l'économie allemande a connu des faiblesses au cours des années écoulées, elle est toujours restée structurellement compétitive, ce qui a permis à l'Allemagne de résister aux grandes mutations et lui permettra, sous réserve qu'elle parvienne à moderniser ses équilibres institutionnels et ses politiques de développement structurel, de préserver sa dynamique dans un environnement de plus en plus concurrentiel.

\section{Emploi dans l'industrie manufacturière de haute et de moyenne-haute technologie en Europe (en \% de l'emploi total, 2004 - NUTS 2)}

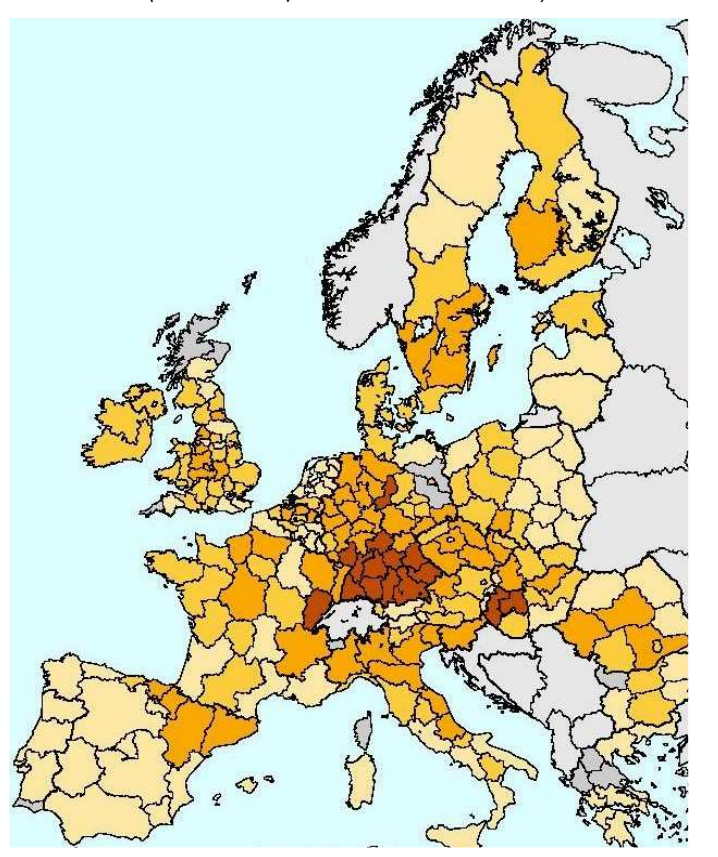

Source : Eurostat, Eurographics (C) Communautés européennes, 2006. NB : les régions en noir ont une part supérieure à $12,5 \%$; dans les régions les plus claires, cette part est inférieure à $5 \%$ ou les données ne sont pas disponibles.

Pays aux 9 frontières, territoire structuré par des voies de communication naturelles favorisant l'ouverture sur le monde (Rhin, Main, Danube, Elbe), la République fédérale a développé une structure et une culture économiques foncièrement axées sur les échanges. Le fort degré de son 
ouverture à l'international, tel que le révèle le fait qu'elle soit championne du monde à l'export depuis plusieurs années, mais bien plus encore le fait qu'elle soit parfaitement insérée dans le partage mondial et européen du travail en est le reflet: l'Allemagne exporte autant de biens intermédiaires et de biens d'investissement qu'elle en importe. Sachant qu'elle vend et achète pareillement les deux tiers de ses produits dans l'UE, sa place centrale dans une Europe à désormais 27 Etats membres présente en ce sens un atout majeur.

A cela s'ajoute une structure sectorielle de l'industrie dans laquelle quatre branches prédominent traditionnellement et ce, depuis les années 1990 : l'automobile (avec un taux d'exportation de près de $60 \%$ ), la construction mécanique (plus de $50 \%$ ), l'électrotechnique ( $40 \%$ ) et la chimie (plus de $50 \%$ ). A elles quatre, ces branches réalisent plus de $40 \%$ des exportations et un tiers des importations allemandes. Si elles sont les plus ouvertes au partage mondial du travail, elles sont aussi les plus intensives en savoir et en R\&D. Autrement dit, cette économie industrielle se caractérise par une spécialisation dans les moyennes et hautes technologies. En outre, dans l'espace européen, ces activités se concentrent fortement en Allemagne - essentiellement dans le sud, sur l'axe qui va de Francfort/Main au Lac de Constance à la frontière suisse. Une région se distingue de loin : celle de Stuttgart (BadeWurtemberg), où la part de l'emploi dans ce segment atteint 22,2 \% (2004, Eurostat), alors que la moyenne européenne (UE 25) n'est que de 6,9\%.

\section{Répartition territoriale des secteurs à forte croissance en Allemagne} (en \% de l'emploi)

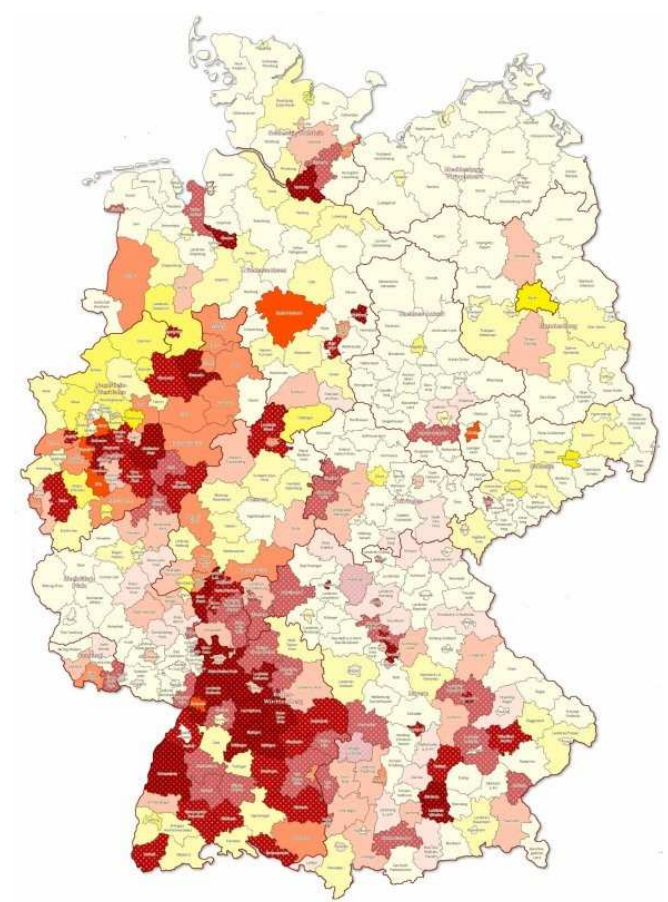

Source : Prognos AG, Zukunftsatlas 2006 (carte « Starke Branchen - starke Regionen »). NB : cette carte présente les 439 villes et districts d'Allemagne selon leur degré de spécialisation (clusters) dans 14 branches ou secteurs à forte croissance (données 2000/04). Les zones les plus sombres sont celles qui présentent le plus fort taux d'emploi dans ces secteurs. 
Le long du même axe se concentrent par ailleurs d'autres secteurs, comptant parmi les plus dynamiques, comme l'industrie aéronautique et spatiale, l'industrie pharmaceutique, les technologies médicales, la logistique, les TIC ou encore les services de R\&D. L'industrie aéronautique et spatiale, par exemple, particulièrement intensive en R\&D (les entreprises y consacrent $20 \%$ de leur CA ; Prognos, 2006), se concentre dans deux clusters : agglomérations de Hambourg et, plus encore, de Munich qui est le berceau de ces activités. Deux pôles accueillent également l'essentiel de l'industrie pharmaceutique : Francfort/Main-Darmstadt et Ulm. Les technologies médicales sont, elles, le point fort de Tuttlingen-Tübingen comme de NurembergErlangen. Quant à Francfort/Main et Dortmund-Unna, ces régions sont le fief de la logistique. Dans le domaine des prestations de services de R\&D, deux autres pôles se distinguent: Berlin/Potsdam (dont la bonne douzaine d'universités attire comme un aimant les centres de recherche appliquée) et Dresde (spécialisation : microélectronique).

Or les cartes ci-dessus révèlent aussi l'existence de pôles de concentration d'activités ou clusters dans le nord (Hambourg, Hanovre-Brunswick-Salzgitter) ou l'est de l'Allemagne (Dresde et Berlin-Potsdam). Cela s'explique par leur localisation sur les grands axes de communication. Car dans ce pays sans ressources naturelles notables (exception faite du bassin houiller de la Ruhr qui fut, du milieu du XIX ${ }^{\mathrm{e}}$ siècle jusqu'à la fin des années 1960, l'un des berceaux de l'industrialisation allemande), l'industrie et les services se sont depuis toujours installés au plus près des grands fleuves (Rhin, Danube, Elbe, Main, Ruhr, Oder, Ems, etc.) qui traversent l'Allemagne, lui ouvrant des débouchés sur la mer du Nord, la Baltique ou la mer Noire. Ils sont reliés entre eux par un système transversal de canaux, dont le canal MainDanube qui, via l'affluent du Rhin qu'est le Main, relie les axes du Rhin et du Danube, ou ce Mittellandkanal (conçu en 1856 et dont la construction s'est achevée à la fin des années 1930) qui relie l'Ems à l'Oder et qui, long de $326 \mathrm{~km}$, est un axe de navigation fluviale d'une importance stratégique.

Ce sont donc les fleuves et canaux (7 $500 \mathrm{~km}$ de voies navigables) puis, plus récemment, les plateformes aéroportuaires (quelque 500 dont plus de la moitié a un rôle décisif pour l'économie locale), qui structurent l'espace économique allemand, lui assurant une fluidité d'autant plus grande que le territoire est densément maillé d'autoroutes (12 $400 \mathrm{~km})$, à quoi il faut ajouter l'équivalent de nos nationales $(231500 \mathrm{~km})$. Sans parler des voies de chemin de fer (38 $000 \mathrm{~km}$ pour le seul réseau de la Deutsche Bahn) héritées du gigantesque effort de construction d'infrastructures modernes de la Prusse et de l'empire bismarckien : la première ligne, Nuremberg-Fürth, longue de 6 km, avait été mise en service en 1835.

Les principaux pôles d'activité ou clusters se situent aujourd'hui sur les points nodaux, à l'interconnexion de toutes ces voies. Si un Land comme la Hesse présente une économie dynamique bien qu'il n'ait pas d'industrie notable, cela est dû à sa situation dans la région Rhin-Main-Neckar comme à l'importance de l'aéroport de Francfort, premier d'Allemagne (avec quelque 53 millions de passagers en 2006) et d'Europe pour le fret (plus de 2 millions de tonnes en 2006). Si le bassin de la Ruhr (Rhénanie du Nord-Westphalie) est toujours une zone industrielle de premier plan malgré l'obsolescence de son ancienne monoculture industrielle, cela est dû à son emplacement au confluent de la Ruhr et du Rhin, à quelques encablures de Rotterdam : le port fluvial de Duisburg est le premier mondial, avec quelque 50 millions de tonnes de fret transbordées (2006). Et si l'agglomération de Munich est parvenue à se hisser au rang des régions les plus compétitives d'Europe, elle le doit à sa situation sur l'Inn, affluent du Danube, et à l'extension systématique de son aéroport. Quant au retard pris 
dans la construction du grand aéroport qui desservira la capitale, il permet de comprendre pourquoi la région de Berlin-Potsdam est loin d'avoir épuisé son potentiel.

\section{Le polycentrisme, principal ressort de la compétitivité}

Cette structuration de l'espace, ajoutée au fait que le nord-ouest de l'Allemagne a longtemps été coupé artificiellement de ses zones d'échanges naturelles à l'est par la partition de l'Europe, explique pourquoi ce sont les Länder situés au sud du Main qui se taillent la part du lion en ce qui concerne les industries performantes ou à fort potentiel : le Bade-Wurtemberg et la Bavière majoritairement. Ces régions sont, de ce fait, celles qui enregistrent le plus fort taux de salariés occupés dans la R\&D en Allemagne comme, plus généralement, en Europe : "parmi les régions en pointe qui comptent plus de $2 \%$ du total des personnes travaillant dans la $R \& D$, près de $40 \%$ sont situées en Allemagne ", relève en effet Eurostat (op.cit.). Et la part du PIB consacrée à la $\mathrm{R} \& \mathrm{D}$ y est non seulement supérieure à la moyenne européenne ou allemande (2,5\%), mais elle dépasse aussi de loin l'objectif de Lisbonne (3\%) : le BadeWurtemberg se classe même au rang de champion mondial, avec un taux de 3,9\%, devant la Suisse, les USA, la Finlande, le Japon et le Danemark, comme le révèle par ailleurs une étude réalisée par l'institut DIW pour le compte de la Fondation Deutsche Telekom et la Fédération allemande de l'industrie BDI (Innovationsindikator Deutschland 2006). Son point fort est la recherche appliquée et le travail en réseaux de partenariats rassemblant entreprises, acteurs de la recherche fondamentale, appliquée et acteurs institutionnels (CCI).

Plus généralement, cette culture des réseaux de partenariats, qui inclut d'intenses activités de transfert des savoirs (le Bade-Wurtemberg est exemplaire à cet égard), est un autre de ces facteurs structurant la compétitivité de l'économie allemande. Selon les Länder, cette culture est plus ou moins prononcée certes, puisqu'elle repose sur la structuration historique des activités comme l'approche qu'ont les pouvoirs publics de leur politique régionale. Mais par-delà ces différences d'ordre plutôt culturel, cette logique du partenariat qui se trouve au cœur du système d'innovation national est l'application à un certain type d'activités du mécanisme-clef sur lequel repose l'ensemble du modèle économique et social allemand. Il est fondé sur le principe de subsidiarité dans son acception première : le rôle principal revient à l'acteur économique, l'Etat se contentant de veiller à offrir un cadre favorable aux activités.

\section{Le principe de subsidiarité au cœur du libéralisme organisé}

Le principe de subsidiarité est bien plus ancien que le laisse entendre son premier emploi officiel par le Pape Pie XI dans son Encyclique Quadragesimo Anno (1931). Et son acception dépasse de loin la doctrine sociale de l'Eglise catholique, comme le rappelle le constitutionnaliste Roman Herzog (Herzog, 2006). Ce principe «signifie que le pouvoir décisionnel doit toujours être placé au plus près des acteurs concernés (on pourrait dire aussi : au plus près des problèmes constatés) ». Présenté sous un autre angle, il implique une sorte de règle de 'charge de la preuve' : "Si on veut décider quelque chose 'd'en haut', au niveau central, il faut d'abord fournir la preuve que cette décision ne peut pas être prise 'en bas'”.

Voilà le principe qui régit aussi bien l'organisation de la société que de l'Etat en Allemagne : « cet 'en bas' ne s'arrête pas aux Länder: les communes, la société civile et ses organisations, et plus encore les citoyens eux-mêmes sont le 'siège' premier des compétences décisionnelles. Dans une société démocratique, le pouvoir de décision se délègue toujours du bas vers le haut, jamais dans le sens inverse ».

Dans l'acception large allemande, ce principe fonde pareillement l'organisation démocratique de l'économie, car il repose sur la liberté des acteurs et la diversité de leurs choix. "Si, d'un côté, la diversité est à la fois l'expression et la condition de la liberté, de l'autre, sa force productive repose sur la concurrence qu'elle génère dès lors qu'on la comprend comme la 'diversité des solutions proposées'”. 
Dès lors, cette subsidiarité va de pair en Allemagne avec un polycentrisme prononcé qui se présente sous plusieurs aspects. Il est d'abord territorial, dans la mesure où les activités économiques se concentrent dans une série de pôles historiques régionaux : la Saxe, qui fut un des berceaux de l'automobile (l'autre étant le Bade-Wurtemberg), l'est redevenue après l'Unité ; la région de Wolfsburg en Basse-Saxe l'est restée depuis l'implantation de l'usine Volkswagen sous Hitler; la chimie de Rhénanie du Nord-Westphalie, aujourd'hui modernisée, a survécu à la guerre ; la logistique s'est développée dans la région Rhin-Ruhr sur la base des infrastructures existantes, aujourd'hui revalorisées par la position centrale de la région au cœur de l'espace européen, etc. D'une manière ou d'une autre, les entreprises œuvrant dans ces champs sont amenées à coopérer entre elles et avec leur réseau de soustraitants, voire avec un dispositif de recherche lui aussi réparti sur l'ensemble du territoire.

A ce polycentrisme des centres de décision économiques s'ajoute celui des pouvoirs. La société civile allemande étant organisée, elle participe du processus de prise de décision, que ce soit sous la forme institutionnalisée de la «triade décisionnelle » associant à l'échelon fédéral partenaires sociaux et pouvoirs publics, ou sous celle de diverses variantes à l'échelon du Land ou de la commune. La prise de décision, outre-Rhin, est toujours collective.

La structure fédérale de l'Allemagne est une autre facette, politique et administrative, de ce polycentrisme. Elle aussi (contrairement à d'autres formes de fédéralisme) est établie sur le mode du partenariat : le modèle allemand est celui du fédéralisme coopératif. Il présente deux caractéristiques essentielles.

La première réside dans le principe de l'équilibre des forces entre l'autonomie/concurrence des collectivités territoriales constitutives de la République fédérale (Länder et, à l'échelon inférieur, communes) et la solidarité qui, en liant ces collectivités entre elles, mais aussi à l'échelon supérieur de la fédération (le Bund), crée la cohésion de l'entité RFA. Pour le dire autrement: les Länder ont des droits, mais aussi des devoirs les uns vis-à-vis des autres comme envers le Bund; celui-ci a pareillement des droits et devoirs envers eux. La seconde caractéristique réside dans la forme concrète que prend cet équilibre, à savoir une double répartition des compétences entre Bund et Länder (ce principe s'applique aussi, mais à un degré moindre, entre Land et communes) : à la fois sectorielle (le Bund est seul compétent en matière de sécurité et d'affaires étrangères, les Länder sont souverains pour l'éducation et la culture) et fonctionnelle (le pouvoir administratif appartient aux seuls Länder). Néanmoins, la plupart des compétences sectorielles sont partagées entre ces deux échelons : cette «compétence législative concurrente» (konkurrierende Gesetzgebung) autorise les Länder à légiférer sur leur territoire dans une matière si le Bund n'a pas fait usage de son droit, par exemple dans les domaines de l'assistance sociale, de l'énergie nucléaire, de l'environnement, de la politique de l'habitat ou encore de la navigation maritime ou fluviale.

Cette architecture respecte certes la subsidiarité des Etats membres de l'Allemagne, mais, conjuguée à des tendances au long cours comme celle à la (re)centralisation, avait fini par brouiller au fil des décennies la délimitation des compétences. La réforme du fédéralisme politique intervenue en 2006 dans le cadre de la grande coalition a apporté quelques clarifications, reversant notamment dans le champ de souveraineté des Länder un certain nombre de compétences sectorielles, dont les horaires d'ouverture des magasins. Dans d'autres domaines, même ceux où le Bund a fait usage de sa prérogative d'adopter une réglementation cadre (Rahmengesetzgebung), les Länder ont désormais la possibilité d'adopter des dispositions dérogatoires, principalement dans le domaine de la planification territoriale (Raumord- 
nung), à l'intersection entre politique des transports (infrastructures), aménagement du territoire, urbanisme ou protection de l'environnement. S'ils doivent toujours coordonner leurs plans avec celui du Bund, les Länder, déjà largement autonomes en la matière, vont pouvoir se livrer à une concurrence des sites plus affirmée. Un des principaux outils dont ils disposent depuis 2007 est ainsi par exemple la 'débureaucratisation' des procédures de délivrance des permis de construire.

\section{Un fédéralisme en cours de rénovation}

Cette réforme n'a pas modifié les dispositions cruciales pour l'équilibre des pouvoirs et la cohésion territoriale. Ainsi, l'éventail des «tâches communes » (Gemeinschaftsaufgaben) a été réduit (la construction d'universités ou de CHU revient désormais en propre aux Länder, de même qu'une grande partie du droit de l'environnement), mais le cœur du principe a été maintenu. Il impose au Bund de concourir financièrement à l'accomplissement des tâches des Länder si celles-ci ont une portée d'intérêt général et plus particulièrement si ce soutien " est nécessaire à l'amélioration des conditions de vie » (art. 91a de la Loi fondamentale). Ce concours du Bund s'applique donc toujours à l'amélioration des structures agricoles, à la protection des côtes, et, surtout, à "l'amélioration de la structure économique régionale ».

Conjuguée à l'autonomie budgétaire des Länder et aux mécanismes de solidarité du fédéralisme financier (Finanzausgleich) qui assure à tous les Länder, quel que soit leur poids économique, une capacité financière équivalant à quelque $95 \%$ de la moyenne allemande, cette participation financière du Bund au titre des tâches communes a permis et permet toujours aux Länder de mettre en œuvre des politiques structurelles régionales originales. Ils bénéficient parallèlement du Fonds ERP issu du Plan Marshall : en 60 ans d'existence, 125 milliards $€$ ont ainsi été destinés au soutien au Mittelstand, ces PME qui sont la base même de la culture entrepreneuriale et du tissu industriel allemands et dont la compétitivité a ainsi été soutenue durablement, tant au plan fédéral que régional. Ce Fonds ERP a bien entendu bénéficié également aux nouveaux Länder après l'Unité (45 milliards $€$ de 1990 à 2006), aidant à la reconstruction de ces PME qui avaient massivement dépéri sous le régime de la RDA, parallèlement aux fonds des « Pactes de solidarité » de l'Aufbau Ost destinés aux infrastructures ou à l'aide à l'investissement (261 milliards $€$ pour la période 1995-2020). S'ajoutent à cela, pour les régions en restructuration, à l'est comme à l'ouest, les fonds structurels européens (fonds EFRE surtout : 30 milliards $€$ pour 2000/06) et, plus récemment, ceux des grands programmes de recherche et d'innovation technologique.

Dans ce contexte, tout l'art consiste pour chaque Land à concevoir son propre projet de développement territorial et à mêler intelligemment, lors de sa mise en œuvre, fonds extérieurs et ressources propres. Il en va de son identité comme de son positionnement dans la concurrence entre les Länder qui se trouve avivée aujourd'hui, notamment du fait de la globalisation, par une mobilité croissante des facteurs, dont ces ressources humaines à l'importance stratégique dans l'économie du savoir.

Mais cette approche de la cohésion, fondée sur le principe de solidarité, a connu des dérives. L'impératif constitutionnel de réaliser des conditions de vie équivalentes sur l'ensemble du territoire a abouti, dans certains cas, à des 'rentes de situation', réduisant les effets bénéfiques de l'émulation des sites. Les tâches communes, par exemple, adoptées durant les riches années suivant le «miracle économique » pour lisser des disparités territoriales alors 
résiduelles, ont été étendues depuis, menant à une généralisation des aides. Ces dernières années, un changement de paradigme est intervenu outre-Rhin sur cet aspect comme dans le domaine social au demeurant: désormais, on commence à accepter les disparités territoriales, à l'est comme à l'ouest (remarquons toutefois qu'elles sont moins prononcées qu'en France), et à se départir de l'interprétation égalitariste et redistributive qui avait dénaturé au fil du temps le principe d'équité des chances sous-jacent à la solidarité. "Il faut se rendre à l'évidence : il existe et il a toujours existé dans notre République de grandes disparités dans les conditions de vie. Elles s'étendent du nord au sud comme de l'ouest à l'est. Si on cherche à les réduire, on confortera un Etat distributeur de subventions et on fera porter aux jeunes générations une dette d'un poids insupportable. Nous devons abandonner cette acception de l'Etat qui subventionne tout. Il importe au contraire de faire en sorte que les individus disposent des espaces de liberté nécessaires à la genèse des idées et des initiatives ». C'est ainsi, par exemple, que le président de la République, l'économiste Horst Köhler (il était auparavant président du FMI), s'exprimait peu après sa prise de fonction dans une interview accordée au magazine Focus (13-09-2004).

Il est vrai que le caractère quasi-automatique, rarement ciblé et souvent source d'effets d'aubaine, de ces soutiens a non seulement contribué à retarder l'essor des économies estallemandes (sauf celle de la Saxe qui avait pris très tôt ses distances), mais explique aussi les performances modérées ou la faible dynamique de modernisation d'un certain nombre de Länder de l'ouest. Les exemples d'une mauvaise allocation des fonds de développement structurel sont nombreux. Le Brandebourg, par exemple, avait longtemps tenté de lutter contre la concentration des activités, notamment de haute technologie, dans la ceinture de Berlin, ce qui s'était traduit par des investissements massifs, mais à perte, dans des projets excentrés comme le circuit automobile Lausitzring, un site de production de puces électroniques jamais achevé à Francfort-sur-Oder ou le projet Cargolifter. Même la Bavière, d'habitude citée en exemple, a concentré les moyens sur la seule agglomération de Munich, la transformant certes en un des pôles leaders en Europe, mais au détriment de la restructuration du tissu industriel obsolète de Franconie ou du développement des marges limitrophes de l'ancien Rideau de Fer.

Or « si les gouvernements des Länder ont la possibilité de se présenter sous leur meilleur jour grâce à des projets réussis ou de riches infrastructures, ce ne sont pas eux qui assument les conséquences financières de leurs erreurs, mais l'ensemble des Länder et le Bund », explique ainsi Klaus-Heiner Röhl, spécialiste de la politique du Mittelstand et des politiques structurelles régionales à l'Institut der Deutschen Wirtschaft, centre de recherche proche de la Fédération de l'industrie BDI (Röhl, 2007). Et il cite en exemple l'échec d'un ambitieux parc d'attraction doublé d'une cité des sciences et d'un centre commercial à Brême ("Space Center ») : la ville-Etat y avait investi 300 millions $€$ sur les subsides que lui avait versés le Bund pour lui permettre en réalité de surmonter sa « situation de crise budgétaire extrême » (extreme Haushaltsnotlage) ; comme elle est surendettée, le Bund est tenu de continuer à l'aider.

Un exemple plus récent, et particulièrement flagrant, de ces dérives des mécanismes de solidarité, est la situation budgétaire du Land de Berlin, lui aussi en déficit chronique. Il avait saisi le Tribunal constitutionnel fédéral pour qu'il arbitre le contentieux qui l'opposait au Bund depuis 2003 : arguant des précédents constitués par les Länder de Brême et de Sarre, soutenus financièrement par le Bund, il s'estimait de droit bénéficiaire comme eux de 
l'aide du Bund; or ce dernier refusait. La Cour de Karlsruhe a rejeté la plainte de Berlin dans un arrêt rendu le 19 octobre 2006 (réf. 2 BvF 3/03), estimant que l'origine des difficultés budgétaires de Berlin "se situe plutôt du côté des dépenses que de celui des recettes». Plus généralement, l'arrêt pointe une contradiction foncière dans la forme qu'a prise au fil du temps l'organisation financière de la République fédérale : la part croissante de l'intervention du Bund, alimentée par les revendications en hausse de Länder peu rigoureux dans leur gestion (Bourgeois, 2006). Cette pratique est en effet en conflit « avec le principe selon lequel l'autonomie politique des Länder implique que ceux-ci en gèrent, de manière autonome et responsable, les conséquences budgétaires ».

Cela étant, dans l'écrasante majorité des cas, la liberté qu'ont les Länder de définir leur propre politique économique et structurelle (dont la construction/extension d'aéroports) - y compris dans le cadre des missions relevant des tâches communes -, de même que leur lutte pour l'attractivité des sites (elle inclut la qualification des ressources humaines et la valorisation du cadre de vie), dope leur croissance et leur compétitivité et, partant, leur attractivité pour les investisseurs. Si on passe en revue les politiques menées par les Länder, on s'aperçoit que, malgré les dérives qu'il a connues dans son application concrète, le fédéralisme à l'allemande, c'est-à-dire une large autonomie d'action de territoires mis en concurrence, mais liés par le principe de solidarité, est un autre de ces facteurs structurels qui fondent la compétitivité de l'économie allemande.

\section{L'européanisation croissante amène une inflexion des politiques structurelles régionales}

Mais alors que, à l'origine, la politique régionale allemande n'impliquait que deux acteurs étatiques (le Land concerné et le Bund), elle est de plus en plus largement déterminée désormais par un troisième acteur : l'échelon européen. L'évolution s'est faite insidieusement, posant un défi de gouvernance politique à l'Allemagne en révélant les contradictions internes de la forme qu'a prise son organisation fédérale. D'abord, au fur et à mesure que progressait l'intégration européenne, et tout particulièrement après la signature du Traité de Maastricht en 1992, les Länder ont dû transférer à l'échelon communautaire une partie de leur souveraineté, notamment en matière de formation ou de politique environnementale, ce qui contribue à déséquilibrer la répartition interne des pouvoirs.

Or, plus généralement, ce Traité a aussi contribué indirectement à paralyser l'évolution du modèle fédéral allemand. Sa ratification avait amené une révision de la Loi fondamentale, ce texte qui non seulement garantit les droits fondamentaux et définit les institutions, mais qui organise également la répartition des pouvoirs dans la République fédérale. La révision avait donné lieu en décembre 1992 à une nouvelle formulation de l'art. 23, transformant cet article grâce auquel s'était faite l'unité allemande (et supprimé ensuite, puisqu'il n'avait plus lieu d'être) en l'article ancrant dans la Constitution allemande l'intégration de la République fédérale dans l'Union européenne instituée par le Traité de Maastricht. S'il permet les transferts de souveraineté du Bund comme des Länder à l'échelon de l'UE, il organise également les pouvoirs de co-décision du Bund, du Bundestag et du Bundesrat en matière d'affaires européennes. Et s'il stipule en deux endroits que «la responsabilité de la Fédération [Bund] pour l'ensemble de l'Etat [allemand] doit être préservée » (art. $23 \S 5$ al. 2 et $\S 6$ al. 2), il impose en contrepartie au gouvernement fédéral un devoir d'information systématique du Bundesrat en amont de toute décision, accordant aux Länder de larges pouvoirs de co- 
décision dans les domaines relatifs aux compétences partagées. Et, «lorsque des pouvoirs exclusifs de législation des Länder sont concernés de manière prépondérante, l'exercice des droits dont jouit la République fédérale d'Allemagne en tant qu'Etat membre de l'Union européenne doit normalement être transféré par la Fédération à un représentant des Länder désigné par le Bundesrat» (art. $23 \S 6$ al. 1 dans le version de 1992).

En réalité, ces nouvelles dispositions se contentent d'intégrer la donne européenne dans le système à deux niveaux du fédéralisme allemand, sans rien changer à la configuration de celui-ci. "La logique intra-allemande de la répartition des compétences a été transposée aux compétences [respectives] en matière d'affaires européennes... Et les structures entrelacées préexistantes du système fédéral ont simplement été doublées " (Eppler, 2006). Cette situation nourrit une rivalité Bund/Länder exacerbée, tant au plan intérieur qu'à l'échelon communautaire, où les Länder ont leurs propres représentations. Or la crispation sur les prérogatives respectives a eu pour effet, lors de la négociation de la réforme du fédéralisme politique adoptée en 2006, de mettre entre parenthèses la révision de fond, pourtant prévue à l'origine, de l'art. 23. L'unique modification adoptée a porté sur son $\S 6$ al. 1, désormais rédigé ainsi : "lorsque des pouvoirs exclusifs de législation des Länder sont concernés de manière prépondérante dans les domaines de la formation scolaire, de la culture, de la radio et de la télévision, l'exercice des droits que possède la République fédérale d'Allemagne en tant qu'Etat membre de l'Union européenne sera confié par la Fédération à un représentant des Länder désigné par le Bundesrat » [modifications mises en valeur par nos soins]. Autrement dit, la reconfiguration, voire l'ouverture potentielle du champ de compétences des Länder que préfigurait, du moins en théorie, la rédaction précédente de cet article a été littéralement 'remise en conformité' avec l'architecture du fédéralisme allemand, figeant ainsi l'entrelacs des pouvoirs au lieu de le clarifier.

L'intégration européenne a eu aussi des effets plus directs sur la latitude d'action des Länder sur leur propre territoire. Ainsi, la réglementation communautaire relative aux subventions publiques limite la liberté des Länder pour inciter les entreprises (PME essentiellement) à s'implanter sur leur territoire : toute aide supérieure à $100000 €$ sur trois ans versée à une entreprise est soumise à l'accord de la Commission. Cette disposition a incité les Länder à infléchir leur politique et à donner une plus large ampleur à une autre forme de soutien aux PME et à l'investissement : les programmes de prêts bonifiés proposés par leur banque régionale dédiée au soutien aux entreprises (par exemple la LfA Förderbank Bayern). Ces banques, spécialisées aujourd'hui dans l'aide à la création de 'gazelles' ou l'aide à l'innovation des PME, ont les mêmes missions que la KfW Mittelstandsbank à l'échelon fédéral.

La politique de cohésion territoriale de l'UE a contribué elle aussi à l'inflexion. Les fonds structurels versés à l'Allemagne ont été légèrement réduits depuis l'élargissement de l'UE, les Länder est-allemands ayant un niveau de vie dépassant le critère d'attribution des aides, fixé à $75 \%$ de la moyenne européenne, et la plupart des zones en reconversion de l'ouest dépassant elles aussi ce seuil : ils ne s'élèvent plus qu'à 26,3 milliards $€$ pour la période 2007/13, dont 15 milliards $€$ pour les nouveaux Länder. Plus important, pour garantir la meilleure allocation de ces aides (et pour éviter une 'course aux aides' de la part des Etats membres), l'utilisation de ces fonds est elle aussi soumise au contrôle des subventions, tant à l'échelon fédéral (tâches communes, aides à l'investissement dans les nouveaux Länder) que régional. D'une manière rampante et indirecte, la Commission contribue de la sorte à (co)piloter la politique structurelle et économique menée par les Länder. 
La transparence des décisions, déjà mise à mal par la mixité du financement Bund/ Länder, s'en trouve encore un peu plus brouillée. En retour, cette tendance souligne la nécessité de réformer en profondeur la structure du financement de "l'amélioration de la structure économique régionale » et plaide en faveur d'une re-régionalisation par le transfert des compétences afférentes du Bund aux Länder. Cela contribuerait à rééquilibrer le rapport entre concurrence et solidarité ou, pour le dire autrement : entre autonomie des collectivités constituantes et unité fédérale (entre forces centrifuges et centripètes). $\mathrm{Si}$, sous cet angle, le problème concret de la politique régionale n'a pas trouvé de réponse lors de la réforme du fédéralisme politique de 2006, cette question de fond n'en continue pas moins elle aussi d'alimenter les réflexions sur une réforme ultérieure de l'organisation du fédéralisme allemand (celle du fédéralisme financier est en cours, notamment sous la pression indirecte de l'UEM) afin de l'adapter à la gouvernance multi-niveaux inhérente au processus d'intégration européen.

L'inflexion de la politique des Länder est nourrie enfin par la politique d'innovation technologique de l'UE, notamment dans le cadre de la Stratégie de Lisbonne. Cette dernière détermine, par cascade, les choix régionaux en la matière. Et elle contribue à favoriser une autre tendance, antérieure mais sous-jacente à la politique d'innovation : l'approche de la politique structurelle sous l'angle de clusters (dont l'un des modèles fut la Silicon Valley aux USA), que ces pôles se soient auto-générés au fil de l'histoire (il suffit alors de soutenir leur développement ou leur réorientation), que leur création ait été planifiée par les pouvoirs publics dans l'espoir de 'voir la graine pousser' ou qu'elle ait été guidée par des considérations purement politiciennes visant à ménager les intérêts locaux malgré l'absence totale d'un tissu d'activités (entreprises, dispositif de R\&D) porteur.

Ces deux dernières catégories ne sont toutefois guère représentées outre-Rhin, pour deux raisons : le principe de subsidiarité favorise une approche de bottom up, et les entreprises du cru jouent un rôle déterminant dans la structuration locale des activités, ce qui n'est qu'une autre expression de la responsabilité que leur confère le même principe. La culture allemande du partenariat en réseau entretient tout naturellement elle aussi cette attitude proactive, et ce sont alors les entreprises qui se tournent généralement vers les pouvoirs publics pour les inclure dans la constitution de leur propre réseau de développement et leur demander, le cas échéant, d'exercer à leur tour leurs responsabilités en veillant à entretenir un cadre favorable aux activités. Le Bade-Wurtemberg fourmille d'exemples à cet égard, concernant la réorientation high-tech de ses pôles industriels. La Saxe pour sa part illustre une stratégie de planification vertueuse : la graine a pris sur la base d'une culture industrieuse et d'une tradition industrielle qu'il a suffi de ranimer et d'ancrer d'entrée de jeu dans le high-tech. Le Brandebourg fournit quelques exemples de l'échec des politiques menées en méconnaissance des réalités du terrain.

La politique d'innovation communautaire, a fortiori depuis qu'elle est couplée au développement territorial, fait que, aujourd'hui, les mécanismes de soutien au développement économique s'en trouvent réorientés systématiquement vers des pôles de compétitivité hightech et, depuis peu, vers leur mise en réseau réelle ou virtuelle. Le Land qui a le mieux formalisé cette nouvelle approche en l'adaptant à la donne régionale (ce qui lui permet au demeurant de marier utilement les divers instruments de soutien) est la Bavière avec son récent programme associant compétences sectorielles régionales et pôles de technologies-clefs dans des réseaux virtuels de clusters. 


\section{L'européanisation crée un conflit entre approches de bottom up et de top down}

Benchmarking oblige, cette politique s'est trouvée à l'origine d'une question parlementaire déposée à l'automne 2006 au Landtag du Bade-Wurtemberg et portant sur une éventuelle imitation de cette 'meilleure pratique' bavaroise. La réponse du gouvernement du Land fut sans appel : il serait absurde de le faire. D’abord, parce que la logique de réseau est très développée dans le Bade-Wurtemberg dont la force économique repose depuis longtemps sur des clusters sectoriels dynamiques (automobile, construction mécanique...), ensuite que les parties impliquées sont rompues à la logique du partenariat, et que l'organisation d'un management de réseau «ne peut être décrété par l'Etat, car il est l'affaire des partenaires euxmêmes ». Enfin, parce que "les clusters naissent toujours indépendamment des frontières géographiques ou administratives lorsque les acteurs sont tous convaincus de la nécessité comme du bénéfice d'une coopération » (31-10-2006, Drucksache 14/499).

Cette prise de position met en lumière, par-delà l'affirmation forte des identités régionales, le pluralisme des approches allemandes, mais aussi les difficultés de mise en œuvre sur le terrain que peut susciter une politique communautaire adoptée dans une logique de top down, bien que s'y mêlent depuis peu quelques éléments d'une approche de bottom up. Ce constat en particulier et, plus généralement, le peu d'ardeur montré par les Etats membres dans la mise en œuvre de la politique globale d'innovation économique et sociale qu'est la Stratégie de Lisbonne avaient amené à une réorientation de cette Stratégie en 2005, qui en simplifie les contours et, plus important, accorde à la Commission de plus larges pouvoirs pour définir des champs d'action. Parallèlement, l'adoption du budget de l'UE pour la période 2007-2013 s'était faite sur la base d'une nouvelle configuration à 25, puis 27 Etats membres, ce qui impliquait entre autres une révision de la politique de cohésion communautaire.

Dans ce double contexte d'un constat de difficile mise en œuvre des objectifs de Lisbonne et de la nécessité de réorienter la politique de développement régional, l'idée a germé de relier entre elles politiques de cohésion et d'innovation. «La Commission a établi-avec l'approbation du Conseil européen - un lien étroit entre le cadre réglementaire de la politique européenne de cohésion d'une part, et l'agenda de Lisbonne révisé d'autre part, en instaurant un rapport clair entre la convergence économique régionale et la modernisation économique », peut-on lire ainsi dans la Communication de la Commission, intitulée "Les régions, actrices du changement économique » (COM[2006] 675 final). Du fait de cette réorientation, la majeure partie des fonds structurels (entre $60 \%$ et $75 \%$ selon les objectifs) obéit désormais à un "fléchage Lisbonne ", c'est-à-dire au ciblage de leur attribution en fonction des critères inhérents à la politique d'innovation technologique. Le budget total de cette nouvelle politique 'régionale' s'élève à 375 millions $€$.

Cette réorientation ne fait pas l'unanimité en Allemagne, même si le gouvernement fédéral d'Angela Merkel développe sa Hightech-Strategie (esquissée par son prédécesseur) selon ces nouveaux axes, proposant entre autres désormais une Initiative Kompetenznetze Deutschland de mise en réseau des pôles de compétitivité. Elle a une double fonction : renforcer la dynamique de compétitivité et l'attractivité internationale du site Allemagne (Hintze, 2006). La critique la plus virulente émane des fédérations professionnelles, principalement de celle de la construction mécanique (VDMA). Dans une prise de position publiée en décembre 2006 en réaction à la nouvelle politique fédérale (et européenne) en la matière, la VDMA estime dans un premier temps que «c'est à juste titre que la politique régionale des Länder 
se concentre de manière accrue sur le soutien des pôles de croissance régionaux plutôt que de poursuivre une politique 'd'arrosage' systématique », par nature indifférenciée et vouée à l'échec. Mais c'est, dans un second temps, pour mieux définir ce que devrait être une politique de pôles de compétitivité efficiente. "En tant que politique régionale, celle-ci est l'affaire des Länder... Dans sa politique de soutien, le Bund peut à la rigueur se consacrer à des clusters virtuels ». Ensuite, le soutien aux clusters régionaux et aux réseaux d'innovation supra-régionaux " doit viser les structures déjà existantes ». Quant à la définition thématique des objectifs, elle doit " suivre par principe une approche de bottom up ». Enfin, au niveau de l'organisation, le choix de l'administrateur ou pilote du cluster doit répondre aux critères "de compétence sectorielle, d'indépendance par rapport à l'Etat et de compétitivité. Il convient de préserver le principe de l'auto-organisation ». On voit bien là le conflit foncier qui oppose l'organisation de l'économie allemande, construite sur la subsidiarité, avec la diffusion croissante d'une logique du top down.

Un autre volet de la réorientation de la politique communautaire porte sur le recentrage de « deux instruments existants de la politique régionale européenne : la coopération interrégionale et le programme de développement urbain "(COM[2006] 675 final). Il repose certes sur un travail mené en coordination par les services de la Commission, les Etats membres, les régions et les villes, concrétisé par des réseaux communs cofinancés par l'intermédiaire du FEDER. Mais il préserve le pouvoir de pilotage de la Commission qui « proposera elle-même à ces réseaux un certain nombre de thèmes axés sur la modernisation économique et sur l'agenda de Lisbonne révisé ». Cette " initiative » communautaire donne lieu à la création d'un nouvel instrument de financement : un "objectif de coopération territoriale européenne ». Si la Commission fait appel au volontariat des régions et villes dans le cadre d'un concours visant à récompenser les meilleures pratiques, elle se réserve toutefois le « rôle de chef de file ».

Pour les Länder allemands, déjà soumis à la centralisation rampante des décisions aux mains du Bund, cette montée en puissance du rôle de la Commission comporte en germe le risque d'une dépossession croissante de leur liberté de choix. Cela dit, contraints depuis toujours de développer un policy mix selon les diverses ressources disponibles, les Länder devraient parvenir à composer sans trop de difficultés avec cette nouvelle approche, d'autant plus qu'ils n'avaient pas attendu cette réorientation communautaire pour procéder à une 'régionalisation' de la politique transversale d'innovation allemande et européenne. La longue pratique de la Saxe, du Bade-Wurtemberg ou de la Rhénanie du Nord-Westphalie en est l'illustration. La nouveauté consistera dès lors simplement dans une appropriation plus affirmée d'une logique de réseau, à l'avenir plus franchement parée des couleurs de l'Europe. Mais ce n'est là qu'une question de communication ; en réalité, cette logique est profondément ancrée dans la culture économique allemande.

\section{Les mutations en cours brouillent les frontières des territoires}

Or ces réseaux, pour la plupart nés naturellement au fil du temps avec la structuration des activités sur le territoire allemand, ne s'arrêtent pas aux frontières des Länder ni ne se limitent aux coopérations transfrontières au sein de l'UE. La même approche de mise en réseau, mais sous l'angle de la diffusion de la compétitivité dans la périphérie des agglomérations où se concentre l'activité avait donné naissance au concept des "Régions métropoles 
européennes en Allemagne » que redynamise la nouvelle politique communautaire notamment par son axe de développement urbain. Ces régions se trouvent aujourd'hui au cœur de la nouvelle politique allemande de planification du territoire, commune au Bund et aux Länder.

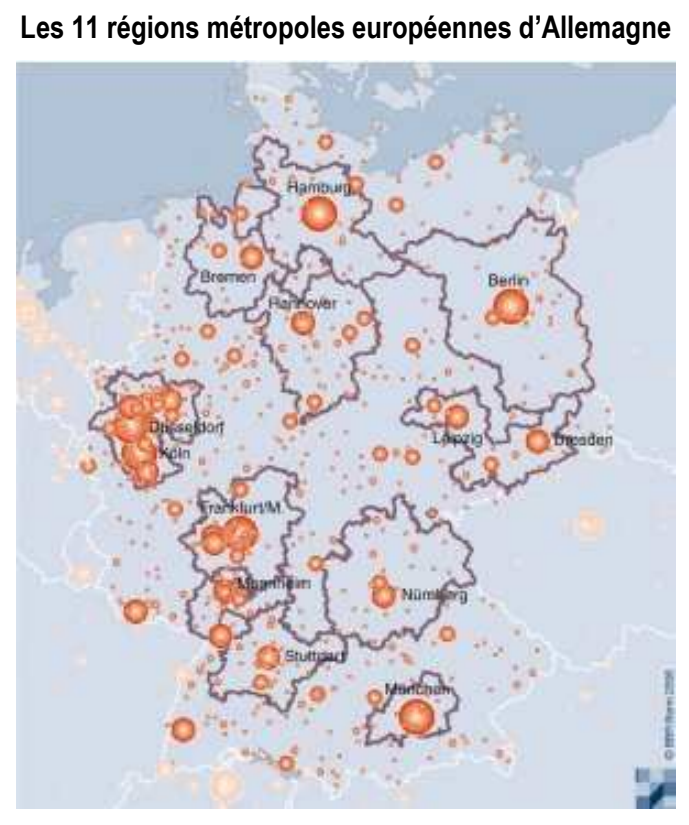

Source : Bundesamt für Bauwesen und Raumordnung (2006).

Leur genèse repose sur l'analyse des grandes mutations affectant le territoire allemand depuis les années 1990 : tendances à la désurbanisation, à la suburbanisation (constitution d'entités déconcentrées, plus petites, à la périphérie des grandes agglomérations) et à l'accroissement de l'écart nord-sud (Pfäfflin, 2005). Contrairement aux apparences, la désurbanisation n'est pas contradictoire avec une autre tendance constatée dans l'Europe entière, la concentration urbaine, à condition de définir cette concentration non pas comme une force centripète, mais comme la diffusion croissante de centres en quelque sorte 'annexes' ; en ce sens, elle intègre la tendance à la suburbanisation, donnant naissance à un nouveau type de découpage territorial. Cette analyse se conjugue au raisonnement suivant, issu de l'observation mais aussi empreint des recommandations développées par les grands cabinets de conseil comme McKinsey (l'un des plus ardents défenseurs des clusters) : les disparités territoriales étant inéluctables, plutôt que de mener une politique de cohésion axée sur les régions les plus faibles, il convient au contraire de soutenir les pôles ou régions les plus dynamiques afin qu'ils puissent rayonner alentour et tirer ainsi le développement de leur périphérie. Dès lors, la Conférence des Ministres en charge de la planification régionale (Ministerkonferenz für Raumordnung) décide en 1995 de définir un nouveau type d'entité régionale (sans le statut de collectivité territoriale) pour les besoins de la planification : la "région métropole ». Ces aires ont toutes pour noyau une de ces zones où se concentrent, outre-Rhin, les activités les plus dynamiques et les plus porteuses. 
Ces régions métropoles, que le Comité des Régions européen désigne depuis sous l'appellation de « régions urbaines fonctionnelles », ont adopté le qualificatif d'européennes en 1999 lors de la Convocation à Porto où s'est constitué l'association METREX rassemblant les 'praticiens' des grandes aires métropolitaines d'Europe. Leur mise en réseau avait été favorisée par le Schéma de développement de l'espace communautaire (SDEC) présenté la même année, une version modernisée du Plan européen d'aménagement du territoire datant des années 1960-70 et incluant divers axes dont la politique des transports (réseaux transeuropéens). A l'instar de la Raumordnungspolitik allemande, il repose sur le principe de cohésion, et « son élaboration part du constat que l'action des Etats membres se complète mieux si elle se base sur des objectifs de développement spatial définis en commun ", explique la fiche de synthèse sur le SDEC publiée par le site de l'Europe. Dans la fiche de présentation de ses activités, le réseau METREX formule ainsi la finalité du SDEC : "Il part de la considération que pour demeurer compétitive dans une économie mondialisée, l'Europe doit arriver à un meilleur équilibre urbain. Il prédit le besoin de regroupements polycentriques de régions et d'aires métropolitaines au-delà du centre économique constitué par l'aire comprenant Londres/Paris/Rhin/Ruhr» (www.eurometrex.org).

La mise en réseau de ces aires urbanisées, préconisée notamment par le nouveau cadre d'orientation de la politique de planification allemande adopté en juin 2006, n'est dès lors que le reflet de cette notion de grappage inhérente à l'approche en termes de clusters. De même, cette approche repose exclusivement sur la capacité à l'auto-organisation des acteurs locaux, autrement dit : sur une logique de bottom up. Quant à la qualification d'européennes de ces régions, elle " ne repose en rien sur une classification ou une catégorie ouvrant droit à des fonds de soutien européens, mais elle exprime la reconnaissance du rôle particulier de ces aires » dans l'espace européen, ainsi que le rappelle leur réseau allemand, l'Initiativkreis Europäische Metropolregionen in Deutschland dans son "Monitoring " régional (2006). Elles sont cependant incluses, sur une base de volontariat, dans la Stratégie de Lisbonne revisitée de l'UE qui n'est, elle, en somme que le prolongement de la même idée cherchant à lier cohésion spatiale et concurrence de sites compétitifs, mais appliquée aux collectivités territoriales constituées. Et dans ce contexte, la logique actuelle du réseau n'est pas sans rappeler cette «méthode ouverte de coordination » (MOC) sur laquelle était construite à l'origine la Stratégie de Lisbonne et à laquelle, faute d'engagement proactif, les Etats membres imputent volontiers l'échec de cette Stratégie. Cette même MOC continue de structurer entre autres la politique sociale à l'échelon communautaire.

Lors de la réunion ministérielle informelle des ministres en charge du développement urbain et de la cohésion territoriale dans les Etats membres et des pays tiers qui s'est tenue à Leipzig les 24 et 25 mai 2007 (l'Allemagne assumait la présidence de l'UE au premier semestre 2007), deux questions étaient à l'ordre du jour : l'adoption de la Charte de Leipzig sur la ville européenne durable et, surtout, la conception du nouvel Agenda territorial de l'UE. Le projet définitif adopté à Leipzig et qu'a transmis l'Allemagne à la Slovénie qui lui succèdera à la présidence de l'UE au premier semestre 2008 pour que cet Etat intègre ce texte dans les travaux du Conseil européen du printemps 2008, décrit ainsi la finalité de cet Agenda : " permettre un développement spatial polycentrique de l'UE et ainsi contribuer à une meilleure utilisation des ressources existant dans les régions » (chapitre I, § 3).

On voit là comment, sous l'effet des tendances multiples liées à la globalisation des activités ou à l'européanisation des politiques, finissent par littéralement se superposer en 
s'entrelaçant des éléments de pouvoirs et des orientations stratégiques avec pour effet de brouiller de plus en plus les champs de compétences et l'identification des niveaux décisionnels. La constitution de réseaux est un élément de réponse à cette donne de plus en plus complexe. Mais si elle est naturelle aux lois de l'économie, elle est délicate à transposer au plan des institutions, puisque pour aboutir, le travail en partenariat implique par définition un partage des tâches entre les diverses parties prenantes, en un mot : une répartition claire des compétences et responsabilités respectives. La structuration de plus en plus protéiforme des processus de prise de décision à l'échelon de l'UE pose avec une acuité nouvelle à l'Allemagne la question de l'adaptation à cette nouvelle donne de ses équilibres institutionnels à deux niveaux, devenus de moins en moins transparents au fil du temps.

L'EUROPÉANISATION DES POLITIQUES RÉGIONALES N'EST PAS À SENS UNIQUE. En effet, " les 'vrais' Etats fédéraux sont en règle générale issus de la réunion d'Etats membres chronologiquement antérieurs à cet Etat fédéral » (Herzog, 2006). Même si les Länder (ouest-) allemands ne sont nés que peu avant leur adhésion à la République fédérale qu'ils constituent et si, lors de la réunification, les Länder est-allemands nouvellement créés après la chute du Mur ont demandé leur adhésion à la RFA conformément à l'art. 23 de la Loi fondamentale qui ouvrait cette possibilité depuis la constitution de la RFA en 1949, ils n'en sont pas moins des Etats membres pleins et entiers de la RFA. Tout comme la RFA est devenue, en vertu du même article, un Etat membre plein et entier de l'UE - à la différence près que, statutairement, l'UE n'est pas une fédération.

Leur statut d'Etat confère aux Länder une souveraineté dont ils ne comptent nullement se départir dans le jeu institutionnel complexe dû à l'intégration européenne. Ils "ne se considèrent pas comme le bras prolongé de la Commission » (Sturm/Pehle, 2006). En réaction à l'emprise croissante de «Bruxelles », ils ont développé une double politique.

La première, à l'échelon fédéral. En application de la nouvelle version de l'art. 23 de la Loi fondamentale (adopté en 1992 et modifié en 2006), une loi régit la coopération Bund/ Länder dans les affaires européennes. En un sens, elle actualise l'Accord de Lindau de 1957 garantissant la co-décision des Länder dans les traités internationaux conclus par le Bund, et grâce auquel entre autres les Länder avaient ouvert, les uns après les autres, une représentation à Bruxelles. En 1992 également a été insérée dans la Loi fondamentale une nouvelle disposition: "pour les affaires de l'Union européenne, le Bundesrat peut constituer une chambre européenne dont les décisions valent décision du Bundesrat » (art. 52 § 3a). Cette nouvelle Europakammer, constituée depuis, confère donc aux Länder, en reflet des mécanismes allemands de co-décision à l'échelon fédéral, également un droit de veto sur les amendements portés à la réglementation européenne dès lors que ceux-ci touchent à leurs compétences. Institutionnellement, le Bundesrat s'en trouve en quelque sorte 'doublé' par cette seconde chambre des Länder, sorte de 'Bundesrat bis' au sein du premier, et qui statue au nom de la RFA pour l'Europe dans les questions relevant de la compétence des Länder. Le lien entre ces deux chambres - et les deux niveaux : fédéral et communautaire - est assuré par la Commission des affaires européennes (EU-Ausschuss) du Bundesrat, le lieu où s'effectue le processus de prise de décision. Si la ratification du Traité de Maastricht s'est traduite par une extension institutionnelle des compétences des Länder vers l'échelon supérieur (l'UE), leurs pouvoirs s'exercent également, et avec une force accrue, vers l'aval (le Land) via l'EU-Ausschuss. Celui-ci voit en effet son rôle "plutôt comme celui d'un précur- 
seur de l'européanisation que d'un gardien des compétences des Länder » (Sturm/Pehle, 2006). C'est donc finalement dans cette instance que se nouent les liens entre les multiples niveaux territoriaux et décisionnels au sein du processus permanent de formation de l'opinion, et donc de l'action politique.

Au plan institutionnel, donc, les Länder ont obtenu une sorte de compensation à la lente décrue de leurs domaines de souveraineté. Or cette participation accrue pèche par un aspect : elle reste trop confinée à l'échelon national et trop peu opérationnelle à l'échelon européen $\mathrm{du}$ fait de la gouvernance multi-niveaux qu'implique l'intégration croissante. Les Länder ont donc parallèlement développé une autre stratégie, plus proactive : leur engagement précoce dans le Comité des Régions à l'échelon européen, une instance consultative dont les Länder allemands - Bavière en tête - ont amplement contribué à asseoir la notoriété et, partant, la montée en puissance dans le jeu institutionnel communautaire qu'entérine la nouvelle Stratégie de Lisbonne. Ce Comité des Régions "défend activement le principe de subsidiarité étant donné que les décisions politiques doivent se prendre au plus près des citoyens », explique ainsi la bavaroise Emilia Müller qui préside la délégation allemande (AdR, 2007).

Malgré tout, les Länder se trouvent comme pris en tenaille entre «Berlin » et "Bruxelles », et leur priorité absolue reste la révision de la répartition des compétences dans le fédéralisme allemand. Or celle-ci achoppe sur trois points.

Le premier réside dans la difficulté à opérer un changement de paradigme dans le fédéralisme financier consistant à réduire, voire à abolir les mécanismes de répartition des ressources; s'ils furent indispensables lors de la reconstruction de l'économie - ou plutôt : des économies - allemandes au sortir de la guerre, ils sont aujourd'hui obsolètes, l'écart entre les niveaux de vie ayant été considérablement réduit au fil des décennies. Se pose également la question de la justification de la participation du Bund dans le financement de certaines tâches comme "l'amélioration de la structure économique régionale », largement déterminée aujourd'hui par l'UE.

Par ailleurs, la responsabilité des Länder dans le cadre du Pacte de Stabilité et de croissance a été accrue par la réforme du fédéralisme politique. Désormais, ils contribuent à hauteur de $35 \%$ au versement de l'amende infligée par la Commission en cas de dépassement du taux de $3 \%$ de déficit (le Bund prenant à sa charge les $65 \%$ restants). Mais cette disposition ne s'applique que lorsque le budget global de la République creuse le déficit. Or depuis 2006, l'Allemagne respecte à nouveau ce critère. Par ailleurs, cette implication des Länder ne suit qu'à la marge le principe du 'pollueur-payeur' : sur la part des Länder, seuls 65 \% du total sont répartis entre les contrevenants; le restant est fonction du nombre d'habitants. De fait, explique le président de l'institut IWD, "seuls 22,75\% des sanctions imputables aux Länder sont établis selon le principe de la pénalisation du responsable » (Hüther, 2007). Les négociations engagées à la fin 2006 sur la réforme du fédéralisme financier sont loin d'être achevées.

Second point. La ratification, en mai 2005 par le Bundestag et le Bundesrat, du projet de Traité constitutionnel de l'UE dont l'un des volets clarifiait les compétences respectives des différents échelons territoriaux au sein de l'UE et donnait de plus larges pouvoirs aux parlements nationaux, s'accompagnait d'un accroissement des pouvoirs d'intervention et d'initiative des Länder dans les décisions communautaires. Ces dispositions ne figurent pas dans la loi de ratification à proprement parler (réduite à l'essentiel), mais dans un ensemble de textes liés, adoptés en cette même occasion, dont une Loi sur l'extension et le renforcement des 
pouvoirs du Bundestag et du Bundesrat (Gesetz über die Ausweitung und Stärkung der Rechte des Bundestages und des Bundesrates in Angelegenheiten der Europäischen Union) qui les implique entre autres dans la procédure de nomination des juges allemands de la Cour européenne de justice (enjeu essentiel en matière de contrôle des aides publiques aux entreprises). De même a été adoptée la prise de position du Bundesrat (avec la réponse du Bund). Ce texte confère notamment aux Länder le droit, au cas où un projet de texte réglementaire émanant de l'échelon fédéral ou communautaire violerait le principe de subsidiarité, d'engager une procédure de blâme auprès du gouvernement fédéral ou un recours auprès de la Cour européenne de justice. Mais l'entrée en vigueur de ces textes, conditionnée à la ratification du Traité constitutionnel par tous les Etats membres de l'UE, s'est trouvée compromise par les « non » de la France et des Pays-Bas.

Le troisième point, enfin, directement lié au précédent, est que « jusqu'à ce jour, Bund et Länder ne sont pas parvenus à développer une vision commune de la manière dont devrait être configuré un Etat fédéral compatible avec l'Europe dans le respect de tous ses échelons » territoriaux (Eppler, 2006). En toute logique économique, cette vision devrait inclure le développement de liens horizontaux entre les Länder, plus nettement institutionnalisés en tout cas que les conférences régulières ou permanentes des divers ministres techniques qui préservent le cloisonnement sectoriel des approches politiques à l'heure où la globalisation et l'européanisation exigent pourtant des approches transversales et, surtout globales. Quant à la Conférence des ministres-présidents, sorte de conférence inter-gouvernementale allemande, elle se limite en général à l'adoption de Contrats d'Etat réglant les questions d'intérêt commun dans leurs champs de compétences exclusifs et ayant force de loi-cadre sur l'ensemble du territoire. La coopération inter-étatique vouée à la mise en réseau des intérêts régionaux est quasi inexistante, d'où aussi la multiplication de réseaux informels.

Mais comment inclure ce nouvel impératif de transversalité dans la Constitution allemande sans bouleverser son organisation avant tout verticale ? Si ce choix sur la forme institutionnelle à donner à un fédéralisme allemand rénové se révèle cornélien pour Bund et Länder, paralysant les réflexions sur la réforme de fond du fédéralisme, le blocage institutionnel européen pour sa part inhibe toute décision sur le rééquilibrage des principes qui le fondent en reportant sine die le choix fondamental entre plus de polycentrisme et de concurrence ou au contraire plus de centralisation et de solidarité.

\section{Indications bibliographiques}

AussChUSS DER REGIONEN (AdR), Die deutsche Ratspräsidentschaft der Europäischen Union und der Ausschuss der Regionen, Bruxelles, janvier 2007 (www.eu2007.de)

Bericht der Bundesregierung zur Zukunftssicherung des Standortes Deutschland, BT-Drucksache 12/5620, septembre 1993 (www.dip.bundestag.de/btd/12/056/1205620.pdf)

BERTELSMANN-STIFTUNG, Die Bundesländer im Standortwettbewerb, 2005 (www.bertelsmann-stiftung.de) Borchard M., MARgedant U. (eds), Der deutsche Föderalismus im Reformprozess, Zukunftsforum Politik, Konrad-Adenauer-Stiftung, Sankt Augustin, 2006

BouRGeOIS I., « Pas d'exception budgétaire pour Berlin », Regards sur l'économie allemande, n 79/2006 Bourgeois I., Gabel M., Stratégie de Lisbonne. Perspectives franco-allemandes, Coll. La France, l'Allemagne et l'Europe (4), Les Notes de l'Ifri, n 59, Paris, avril 2007

BOURGEOIS I., «Quelle politique pour les nouveaux Länder dans l'UE 25 ? », Regards sur l'économie allemande, $\mathrm{n}^{\circ} 61 / 2003$ 
Bundesländer-Ranking, étude réalisée par IW-Consult GmbH et GWS GmbH en coopération avec l'Initiative Neue Soziale Marktwirtschaft et l'hebdomadaire Wirtschaftswoche, octobre 2005 (www.iwconsult.de)

BundeSRAT, Gesetz zu dem Vertrag vom 29 Oktober 2004 über eine Verfassung für Europa, Drucksache 339/05 (Beschluss) du 27-05-2005

BundeSRAT, Gesetz über die Ausweitung und Stärkung der Rechte des Bundestages und des Bundesrates in Angelegenheiten der Europäischen Union, Drucksache 340/05 (Beschluss) du 27-05-2005

CERNAVIN O. et al. (eds), Cluster und Wettbewerbsfähigkeit von Regionen. Erfolgsfaktoren regionaler Wirtschaftsentwicklung, Berlin, 2005

COMITÉ DES RÉGIONS, Avis sur la Communication de la Commission « La stratégie pour la croissance et l'emploi et la réforme de la politique européenne de cohésion. Quatrième rapport d'étape sur la cohésion ", Bruxelles, 11-12-2006, CdR 249/2006 fin EN-JG/AL/CH/ct/id

COMITÉ DES RÉGIONS, Résolution du Comité des régions sur les 'objectifs politiques du Comité des régions pour 2006-2008' ", Bruxelles, 23-02-2006, CdR 11/2006 fin id/nr

COMMISSARIAT GÉNÉRAL DU PLAN / DEUTSCH-FRANZÖSISCHES ISTITUT, Compétitivité globale : une perspective franco-allemande. Rapport du groupe franco-allemand sur la compétitivité, Paris, 2001

COMMISSION DES COMMUNAUTÉS EUROPÉENNES, Les régions, actrices du changement économique, communication de la Commission, Bruxelles, 08-11-2006, COM(2006) 675 final

DESTATIS (ed), Datenreport 2006, réalisé en coopération avec les centres WZB (Berlin) et ZUMA (Mannheim), Bonn, 2006

DELEBARRE M., Stratégie de Lisbonne et cohésion territoriale, au service d'une nouvelle gouvernance européenne, discours prononcé lors de la conférence 'Cohésion territoriale et Stratégie de Lisbonne : exploiter les potentialités des territoires', Amsterdam, 28-06-2006 (www.cor.europa.eu/fr/index.htm)

Deutscher Bundestag, 15. Wahlperiode-175. Sitzung, Berlin, 12-05-2005, BT Drucksache pp. 1634716524, www.bundestag.de/ausschuesse/archiv15/a20/eu_debatte_15_wp/ prot_auszug_15_175.pdf (compte rendu des débats sur la ratification du Traité constitutionnel pour l’ $\overline{U E}$ )

DEUTSCHER BUNDESTAG, Unterrichtung durch die Bundesregierung. Entwurf eines Gesetzes zu dem Vertrag vom 29 Oktober 2004 über eine Verfassung für Europa - Drucksache 15/4900 - Stellungnahme des Bundesrates und Gegenäußerung der Bundesregierung, Drucksache 15/4939 en date du 23-02-2005

DEUTSCHER BundeSTAG, Gesetz zu dem Vertrag vom 29 Oktober 2004 über eine Verfassung für Europa, Entwurf, Drucksache 15/4900 en date du 18-03-2005

DIW BERLIN, Innovationsindikator Deutschland 2006, Forschungsprojekt im Auftrag der Deutsche Telekom Stiftung und des Bundesverbandes der Deutschen Industrie, Berlin, 2006

EMPTER S., VEHRKAMP R. B. (eds), Wirtschaftsstandort Deutschland, ZU Schriften der Zeppelin University zwischen Wirtschaft, Kultur und Politik, Wiesbaden, 2006

EPPler A., « Föderalismusreform und Europapolitik », Aus Politik und Zeitgeschichte, n 50/2006, décembre 2006 [dossier : Föderalismusreform]

Eurostat, Régions : Annuaire statistique 2006. Données 2000-2004, Communautés européennes, 2006

FUEST C., KERVIAN D., Welter P., Wirtschaftliche Freiheit in den deutschen Bundesländern, Friedrich-Naumann-Stiftung, Cologne/Francfort-Main, 2006 (www.fnst.org)

Grote WeStRICK D. et al., Clustermanagement im europäischen Vergleich, Institut Arbeit und Technik im Wissenschaftszentrum NRW, Jahrbuch 2005 (www.iatge.de)

Herzog R., « Kooperation und Wettbewerb », Aus Politik und Zeitgeschichte, n 50/2006, décembre 2006 [dossier : Föderalismusreform]

HintZE P., « Prioritäten setzen. Cluster als Instrument der Wirtschaftspolitik », Rede anlässlich des Kongresses Cluster in der Umsetzung - Lösungen für regionale Innovationspolitik, Berlin, 30-11-2006

HÜTHER M. et al., «Föderalismus in Deutschland : Nach der Reform ist vor der Reform », in INSTITUT DER DEUTSCHEN WIRTSCHAFT (ed), Föderalismus in Deutschland. Ökonomische Analyse und Reformbedarf, Coll. IW Studien, Cologne, 2007

HyPOVEREINSBANK, Fit für die Zukunft? Die Bundesländer im Vergleich, 2007 (www.hvb.de)

INSTITUT DER DEUTSCHEN WIRTSCHAFT (ed), Föderalismus in Deutschland. Ökonomische Analyse und Reformbedarf, Coll. IW Studien, Cologne, 2007

KIESE M., Grundlagen der Clusterpolitik: Begriff, Begründung, Ursachen, Boom, Regional Governance, Seminar zur angewandten Wirtschaftsgeographie im WS 2006/07 : Clusterpolitik in Deutschland, Institut für Wirtschafts- und Kulturgeographie, Leibniz Universität Hannover (www.wigeo.uni-hannover.de)

KÖHLER H., « Jeder ist gefordert », interview accordée au magazine Focus et parue dans l'édition du 13-092004 sous le titre : « Einmischen statt abwenden » (www.bundespraesident.de) 
Landtag Von BADEn-WÜRTTEMBerg, Antrag der Abg. Dr. Reinhard Löffler u.a. CDU und Stellungnahme des Wirtschaftsministeriums - Clusterpolitik, Drucksache 14/499, 23-10-2006 (www.landtag-bw.de/Dokumente)

Leitbilder und Handlungsstrategien für die Raumentwicklung in Deutschland. Verabschiedet von der Ministerkonferenz für Raumordnung am 30. 06. 2006 (www.bmvbs.de)

LichtBlau K. et al., «Erfolgsfaktoren von M+E-Clustern in Deutschland », IW-Trends, n 2/2005

Loi fondamentale pour la République fédérale d'Allemagne du 23 mai 1949 avec les 52 modifications intervenues depuis cette date, texte traduit en français par Autexier C., Fromont M., Grewe C. et Jouanjan O. et téléchargeable sur le site du gouvernement fédéral : www.bundesregierung.de

PFÄFFLIN H., « Paradigmenwechsel in der Regionalpolitik ? Vom Ausgleichsziel zur Förderung von Metropolregionen ", IMU-Akzente, $\mathrm{n}^{\circ}$ 13, décembre 2005 (www.imu-institut.de)

PROGNOS AG, Zukunftsatlas 2004, Zukunftsatlas 2006 : Branchen im Fokus - Wer verfügt über Kompetenzen und Clusterpotenziale? et Zukunftsatlas 2007 (www.prognos.de/zukunftsatlas)

Projektbericht Perspektive-Deutschland 2005/06. Eine Initiative von McKinsey, stern, ZDF und WEB.DE (www.perspektive-deutschland.de)

Regionales Monitoring 2006. Daten und Karten zu den Europäischen Metropolregionen in Deutschland, Initiativkreis Europäische Metropolregionen in Deutschland, 2006 (www.deutsche-metropolregionen.org)

REHFELD D., « Perspektiven der Strukturpolitik nach 2006 », Institut Arbeit und Technik im Wissenschaftszentrum NRW, Jahrbuch 2005 (www.iatge.de)

ReHFeld D., Perspektiven des Clusteransatzes. Zur Neujustierung der Strukturpolitik zwischen Wachstum und Ausgleich, IAT-Report 2005-06 (www.iatge.de)

RöHL, K.-H., « Optionen für die deutsche Regionalpolitik: Mehr regionaler Wettbewerb im Föderalstaat », in INSTITUT DER DEUTSCHEN WIRTSCHAFT (ed), Föderalismus in Deutschland. Ökonomische Analyse und Reformbedarf, Coll. IW Studien, Cologne, 2007

RöHL, K.-H., «Entwicklung und Schwerpunkte der Regionalförderung in Deutschland», IW-Trends, $\mathrm{n}^{\circ} 1 / 2005$

SACHVERSTÄNDIGENRAT ZUR BEGUTACHTUNG DER GESAMTWIRTSCHAFTLICHEN ENTWICKLUNG, Jahresgutachten 1996/97, 1997/98 et 2004/05, Wiesbaden

«SDEC », synthèses de la législation (www.europa.eu/cgi-bin/etal.pl)

Sturm R., Pehle H., Das neue deutsche Regierungssystem. Die Europäisierung von Institutionen, Entscheidungsprozessen und Politikfeldern in der Bundesrepublik Deutschland. 2., aktualisierte und erweiterte Auflage, Wiesbaden, 2006

Territoriale Agenda der Europäischen Union. Für ein wettbewerbsfähigeres nachhaltiges Europa der vielfältigen Regionen, Endgültiger Entwurf (www.bmvbs.de/EU-Ratspraesidentschaft/Arbeitsprogramm-,2708/ Territoriale-Agenda.htm)

Territoriale Ausgangslage und Perspektiven der Europäischen Union. Stärkung der territorialen Kohäsion in Europa unter Berücksichtigung der Lissabon- und Göteborg-Strategien, Diskussionsvorlage für das Generaldirektorentreffen am 15. 032007 in Berlin, Entwurf, 07-03-2007 (www.eu2007.de)

« Une politique de cohésion pour soutenir la croissance et l'emploi : orientations stratégiques communautaires 2007-2013 », synthèses de la législation (www.europa.eu/cgi-bin/etal.pl)

Verband Deutscher MASChINEN- Und ANLAGEnBau, Clusterpolitik auf Bundes- und Landesebene - Position des VDMA, Francfort, décembre 2006

ZimmermanN K. F. (dir.), Deutschland - was nun? Reformen für Wirtschaft und Gesellschaft, Munich, 2006. 
$-1-$

PolyCENTRISME

ET DIVERSITÉ

DES POLITIQUES STRUCTURELLES 



\title{
Compétitivité des territoires : état des lieux et perspectives
}

\author{
Isabelle Bourgeois
}

La croissance du PIB allemand a frisé les $3 \%$ en 2006. Mais il ne s'agit là que d'une moyenne calculée en fonction des apports respectifs des Länder. Largement autonomes en matière de politique économique et budgétaire, ces 16 Etats membres qui composent la République fédérale se différencient par leur poids économique, le profil de leur économie, leur degré de compétitivité, le niveau de leur dette et, bien sûr, le taux de croissance. Les Länder sont en effet bien plus que des entités administratives 'déconcentrées'. Ils sont de véritables Etats fédérés, dotés d'une Constitution, d'un parlement et d'un gouvernement. Et s'ils ne disposent que de peu de compétences législatives exclusives (éducation et culture pour l'essentiel), ce sont eux qui disposent du pouvoir administratif. Et même si un nombre restreint seulement d'impôts leur revient en propre, ils sont également autonomes en matière budgétaire. Autrement dit : ils disposent des moyens et des pouvoirs pour mener leur propre politique structurelle sur leur territoire. Enfin, via le Bundesrat, cette chambre du Parlement allemand où siègent les représentants de leurs gouvernements, ils co-décident de la politique menée par la République fédérale. *

L'Allemagne se caractérise par une grande diversité territoriale. Le clivage est-ouest qui prévalait dans les années suivant l'unification s'est fortement estompé avec la normalisation des économies est-allemandes. Certes, les «nouveaux Länder» pèsent peu dans l'économie allemande, mais cela est dû essentiellement à leur petite taille en comparaison des trois poids lourds que sont la Bavière, le Bade-Wurtemberg et la Rhénanie du Nord-Westphalie : ils contribuent pour une large moitié (56\%) au PIB allemand. Si on y ajoute la Hesse, numéro quatre, cette part s'élève aux deux tiers (65\%). Aujourd'hui, l'Allemagne présente un clivage nordsud plus flagrant que jamais ; il devrait s'accentuer encore dans les années à venir du fait de la position centrale du pays dans l'espace économique européen. Si les régions du sud de l'Allemagne font partie depuis toujours de l'axe de compétitivité ouest-européen qui s'étend de la Grande-Bretagne à Milan en passant par Francfort/Main et Munich, l'intégration des Etats esteuropéens dans l'UE bénéficie à des Länder comme la Saxe et la Thuringe au sud du territoire de l'ex-RDA, proches de la République tchèque et de la Hongrie voisine qui constituent un second axe de compétitivité européen nord-sud en plein développement, à l'est du premier. Les autres régions et Länder d'Allemagne, situés à l'écart de ces grands axes et ne disposant pas (ou moins) de compétences stratégiques pour l'avenir de leur économie, enregistrent des performances moyennes, voire faibles.

Les disparités s'accentuent ainsi entre les dynamiques territoriales, reléguant dans le peloton de queue de vastes ensembles régionaux par-delà les frontières des Länder, qu'ils soient situés à l'est (dans le Mecklembourg et la Saxe-Anhalt, par exemple) ou à l'ouest (Basse-Saxe,

*Version augmentée et actualisée d'un article paru dans Regards sur l'économie allemande, n 81, mai 2007. 
Hesse, Rhénanie-Palatinat). L'Allemagne s'est diversifiée au point que l'institut Prognos, qui a consacré une étude - le Zukunftsatlas 2007 - à la compétitivité des districts régionaux (non des Länder), évoque même aujourd'hui une structure territoriale « à quatre vitesses ».

\section{Etats membres au profil économique très différencié}

Une partie des disparités économiques entre Länder s'explique par la 'construction' de la RFA après 1945. La structuration des entités territoriales et politiques que sont les Länder s'est effectuée progressivement, sur la base d'identités provinciales préexistantes et fortement marquées comme dans le cas de la Bavière ou de Hambourg, mais bien plus encore au gré de la politique des Alliés dans leurs zones d'occupation respectives. C'est ainsi qu'a été créé le minuscule Land de Brême (une ville-Etat : Stadtstaat) : il s'agissait d'une enclave portuaire américaine dans la zone d'occupation britannique. Quant au Land de Bade-Wurtemberg, il a été créé en 1952, après la constitution de la RFA (1949), par voie de referendum, et réunit depuis des régions qui se situaient dans les anciennes zones d'occupation française et américaine. Les Länder est-allemands pour leur part ne sont nés qu'après la chute du Mur, par réanimation des identités provinciales qu'avaient gommées les régimes centralisateurs du $\mathrm{III}^{\mathrm{e}}$ Reich (ce qui valait également pour l'ouest) puis de la RDA. La Saxe et la Thuringe, au statut d'Etat libre (Freistaat) comme la Bavière, en sont les exemples patents. En ce qui concerne Berlin, la partie est, capitale de l'ex-RDA, a été réintégrée dans la ville-Etat de Berlin, aujourd'hui également capitale de la RFA réunifiée.

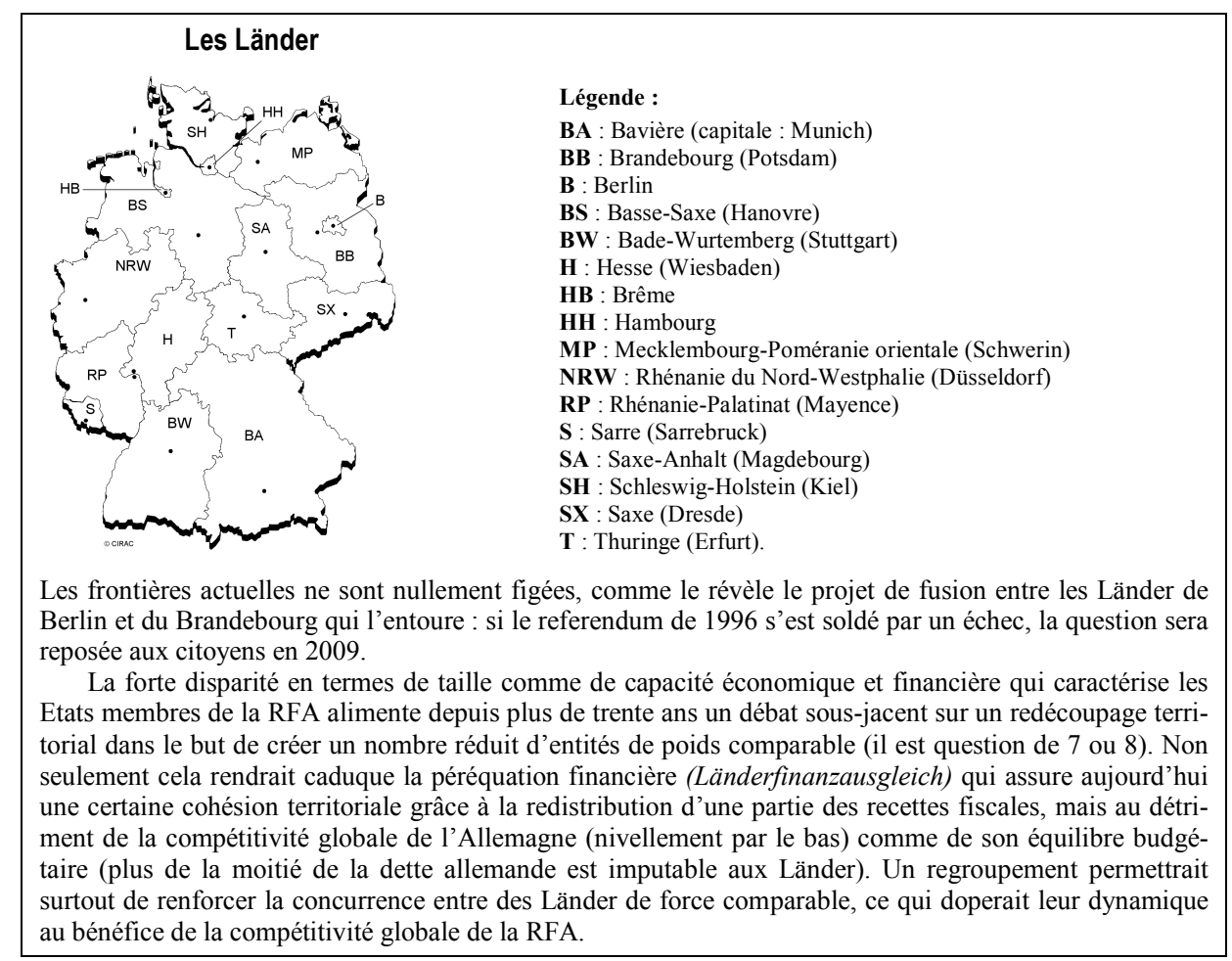


Les 16 Länder de la République fédérale

\begin{tabular}{|c|c|c|c|c|c|c|c|}
\hline & $\begin{array}{l}\text { Superficie }^{\text {a) }} \\
\text { en }^{2} m^{2}\end{array}$ & $\begin{array}{l}\text { Habitants }{ }^{\text {a) }} \\
\text { en millions }\end{array}$ & $\begin{array}{l}\text { Densitéa) } \\
h a b . / \mathrm{km}^{2}\end{array}$ & $\begin{array}{c}\text { PIB }^{\mathrm{b})} \\
\text { en milliards } \epsilon\end{array}$ & $\begin{array}{c}\text { Taux de } \\
\text { croissance }^{\text {b) }}\end{array}$ & $\begin{array}{c}\text { PIB par hab..) } \\
\text { en } \epsilon\end{array}$ & $\begin{array}{c}\text { Part dans } \\
\text { RFA }^{\text {b) }}\end{array}$ \\
\hline Bade-Wurtemberg & 35752 & 10,7 & 300 & 337,2 & $+3,5 \%$ & 31400 & $14,6 \%$ \\
\hline Basse-Saxe & 47624 & 8,0 & 168 & 196,5 & $+2,7 \%$ & 24600 & $8,5 \%$ \\
\hline Bavière & 70552 & 12,5 & 177 & 408,3 & $+2,5 \%$ & 32700 & $17,7 \%$ \\
\hline Berlin & 892 & 3,4 & 3807 & 80,3 & $+1,9 \%$ & 23600 & $3,5 \%$ \\
\hline Brandebourg & 29479 & 2,6 & 87 & 49,5 & $+2,1 \%$ & 19400 & $2,1 \%$ \\
\hline Brême & 404 & 0,7 & 1641 & 25,3 & $+2,8 \%$ & 38100 & $1,1 \%$ \\
\hline Hambourg & 755 & 1,7 & 2309 & 86,0 & $+3,7 \%$ & 49200 & $3,7 \%$ \\
\hline Hesse & 21115 & 6,1 & 289 & 204,1 & $+1,8 \%$ & 33600 & $8,9 \%$ \\
\hline Mecklembourg-Poméranie & 23180 & 1,7 & 74 & 32,3 & $+2,1 \%$ & 19000 & $1,4 \%$ \\
\hline Rhénanie du Nord-W. & 34085 & 18,1 & 530 & 500,7 & $+2,8 \%$ & 27800 & $21,7 \%$ \\
\hline Rhénanie-Palatinat & 19853 & 4,1 & 204 & 100,3 & $+2,6 \%$ & 24700 & $4,4 \%$ \\
\hline Sarre & 2569 & 1,0 & 409 & 28,0 & $+2,0 \%$ & 26700 & $1,2 \%$ \\
\hline Saxe & 18415 & 4,3 & 232 & 88,7 & $+4,1 \%$ & 20800 & $3,8 \%$ \\
\hline Saxe-Anhalt & 20446 & 2,5 & 121 & 50,0 & $+3,8 \%$ & 20400 & $2,2 \%$ \\
\hline Schleswig-Holstein & 15799 & 2,8 & 179 & 69,7 & $+1,7 \%$ & 24600 & $3,0 \%$ \\
\hline Thuringe & 16172 & 2,3 & 144 & 45,8 & $+2,8 \%$ & 19700 & $2,0 \%$ \\
\hline$R F A$ & 357093 & 82,4 & 231 & 2302,7 & $+2,8 \%$ & 28000 & $100,0 \%$ \\
\hline
\end{tabular}

Sources des données : Superficie et habitants : Statistische Ämter des Bundes und der Länder ; PIB, croissance et part du PIB allemand (aux prix courants) : Arbeitskreis Volkswirtschaftliche Gesamtrechnungen der Länder, a) état au 31-12-2005 ; b) part du PIB, 2006 ('́tat ; janvier 2007). NB : chiffres arrondis. 
Structure de l'économie des Länder (2005) - part respective des secteurs (en \%)

\begin{tabular}{|c|c|c|c|c|c|c|c|}
\hline & $\begin{array}{l}\text { Agri- et } \\
\text { sylviculture }\end{array}$ & Industrie & BTP & $\begin{array}{l}\text { Commerce, gastro- } \\
\text { nomie, transports }\end{array}$ & $\begin{array}{l}\text { Crédit, bail, services } \\
\text { aux entreprises }\end{array}$ & $\begin{array}{l}\text { Prestataires publics } \\
\text { et privés }\end{array}$ & $\begin{array}{l}\text { Total PIB } \\
\text { (milliards } \epsilon)\end{array}$ \\
\hline Bade-Wurtemberg & $0,80 \%$ & $34,26 \%$ & $4,16 \%$ & $15,66 \%$ & $26,29 \%$ & $18,83 \%$ & 294,11 \\
\hline Basse-Saxe & $1,85 \%$ & $26,26 \%$ & $4,25 \%$ & $26,26 \%$ & $24,72 \%$ & $23,72 \%$ & 172,61 \\
\hline Bavière & $1,11 \%$ & $26,71 \%$ & $3,93 \%$ & $16,08 \%$ & $31,55 \%$ & $20,61 \%$ & 359,60 \\
\hline Berlin & $0,14 \%$ & $15,24 \%$ & $3,16 \%$ & $16,47 \%$ & $33,69 \%$ & $31,30 \%$ & 71,17 \\
\hline Brandebourg & $2,14 \%$ & $19,69 \%$ & $5,21 \%$ & $18,99 \%$ & $25,66 \%$ & $28,33 \%$ & 43,38 \\
\hline Brême & $0,27 \%$ & $24,60 \%$ & $2,84 \%$ & $25,50 \%$ & $26,63 \%$ & $19,15 \%$ & 22,19 \\
\hline Hambourg & $0,19 \%$ & $15,80 \%$ & $2,02 \%$ & $27,07 \%$ & $37,19 \%$ & $17,71 \%$ & 74,85 \\
\hline Hesse & $0,50 \%$ & $21,34 \%$ & $3,41 \%$ & $19,17 \%$ & $36,91 \%$ & $18,67 \%$ & 181,02 \\
\hline Mecklembourg-Pom. & $2,83 \%$ & $13,37 \%$ & $5,56 \%$ & $21,06 \%$ & $24,81 \%$ & $32,40 \%$ & 28,58 \\
\hline Rhénanie du N.-W. & $0,63 \%$ & $25,61 \%$ & $3,40 \%$ & $18,34 \%$ & $29,28 \%$ & $22,72 \%$ & 439,62 \\
\hline Rhénanie-Palatinat & $1,60 \%$ & $28,04 \%$ & $3,76 \%$ & $17,40 \%$ & $25,21 \%$ & $25,21 \%$ & 88,25 \\
\hline Sarre & $0,24 \%$ & $30,45 \%$ & $4,12 \%$ & $14,84 \%$ & $27,66 \%$ & $22,68 \%$ & 24,73 \\
\hline Saxe & $1,08 \%$ & $22,42 \%$ & $5,66 \%$ & $16,57 \%$ & $27,25 \%$ & $27,03 \%$ & 76,84 \\
\hline Saxe-Anhalt & $1,70 \%$ & $22,57 \%$ & $5,70 \%$ & $21,21 \%$ & $20,66 \%$ & $28,18 \%$ & 43,51 \\
\hline Schleswig-Holstein & $1,66 \%$ & $18,64 \%$ & $3,41 \%$ & $21,68 \%$ & $29,26 \%$ & $25,32 \%$ & 61,85 \\
\hline Thuringe & $1,52 \%$ & $24,83 \%$ & $5,75 \%$ & $16,64 \%$ & $23,09 \%$ & $28,14 \%$ & 40,15 \\
\hline $\boldsymbol{R F A}$ & $0,98 \%$ & $25,45 \%$ & $3,89 \%$ & $18,08 \%$ & $29,24 \%$ & $22,36 \%$ & 2022,47 \\
\hline
\end{tabular}

Source des données : Arbeitskreis Volkswirtschaftliche Gesamtrechnungen der Länder (aux prix courants ; données publiées le 14-02-2007; état : août 2006). NB : calculs effectués par nos soins ; pourcentages arrondis. 


\section{Les Länder industriels ont les meilleurs taux de croissance}

En termes de croissance, le champion est la Saxe, un Land industriel de taille moyenne : elle a enregistré un taux de $+4,0 \%$ en 2006 . L'année précédente, alors que la moyenne allemande s'établissait à $+0,9 \%$, la médaille revenait à la petite Sarre, industrielle elle aussi $(+2,8 \%)$ et elle aussi en restructuration. La riche Hesse, site tertiaire (financier) par excellence et quatrième économie d'Allemagne, présente pour sa part des performances moyennes : $+2,1 \%$ après un maigre $+0,4 \%$ en 2005. C'est que ce Land qui abrite la BCE se trouve pénalisé par le fait que le tertiaire y représente $75 \%$ des activités. Car les services ne s'exportent guère - à l'inverse des biens industriels, ce qui explique pourquoi le Bade-Wurtemberg se classe numéro deux en termes de croissance $(+3,5 \%$ en 2006$)$. Il est non seulement le site industriel allemand par excellence, mais aussi le leader européen de la construction mécanique et donc le leader allemand (la part de la construction mécanique allemande dans l'UE est de $39 \%$ ).

Les Länder se différencient par leur taille, et bien plus encore par la structure de leur économie, déterminante pour leur contribution au PIB allemand. Dans une économie allemande ouverte à l'international, et dont le principal moteur est l'exportation de biens et services industriels, les trois poids lourds industriels que sont la Rhénanie du Nord-Westphalie (au PIB comparable à celui des Pays-Bas), la Bavière et le Bade-Wurtemberg jouent un rôle prépondérant. Ils sont le fief des branches-phares de l'industrie allemande : construction mécanique, automobile, électrotechnique et chimie. Ils se voient dorénavant défiés par la Saxe.

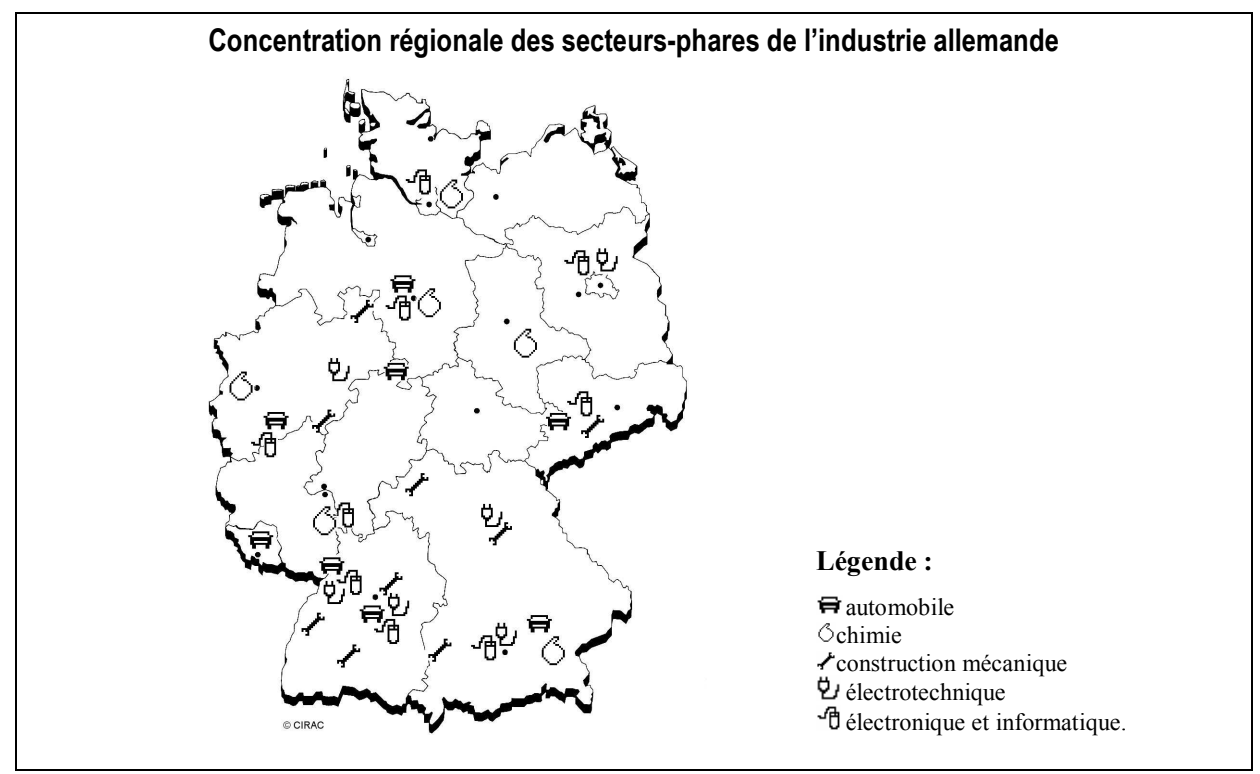

Cette structuration territoriale de l'économie allemande est en grande partie le fruit de l'histoire : la Rhénanie du Nord-Westphalie et le Bade-Wurtemberg à l'ouest, la Saxe et la Thuringe à l'est, sont les berceaux de l'industrialisation allemande ; de par leur profil, ces sites sont toujours spécialisés dans l'automobile, la construction mécanique ou la mécanique de précision et se caractérisent par un dense tissu de PME (même en Rhénanie du Nord- 
Westphalie). Le site de Wolfsburg (Basse-Saxe), pour sa part, connu au XIX ${ }^{\mathrm{e}}$ siècle pour la manufacture Büssing (camions), avait été choisi quasi ex nihilo comme site de production de la future «voiture du peuple» (Volkswagen) par une commission instituée par Hitler en 1937. La Saxe, du temps de la RDA, était au cœur du dispositif de production et de R\&D en microélectronique du régime; les anciens combinats Robotron et Mikroelektronik n'ont pas survécu, mais après l'unité allemande, le tissu d'activités s'est reconstitué sur des bases plus modernes. Il en fut de même de l'optique de précision à Iéna en Thuringe (Zeiss), redynamisée après l'Unité. Quant à la région de Munich, elle doit son rang de métropole de l'électrotechnique à la construction du Mur de Berlin : Siemens, qui y avait son siège, avait alors préféré le transférer en des lieux plus sûrs. Mais l'histoire a jeté aussi les bases de profils aujourd'hui obsolètes, comme le montrent les restructurations en cours dans la chimie en SaxeAnhalt (Bitterfeld, Leuna), ou dans ce qui reste d'industrie charbonnière et sidérurgique lourde dans le bassin de la Ruhr.

\section{Les Länder est-allemands n'ont plus rien de « l'ex-RDA »}

L'Allemagne de l'Est n'est plus, depuis 1990, une entité territoriale et administrative d'un seul tenant. Après l'effondrement de l'Etat centralisé qu'était la RDA, cinq collectivités territoriales largement autonomes se sont constituées, ces «nouveaux Länder » qui ont demandé à intégrer la RFA en application de l'article 23 de la Loi fondamentale - le même art. 23 qui, dans sa version entièrement remaniée après l'achèvement politique de l'unification, ancre désormais constitutionnellement l'Allemagne dans l'Union européenne. L'appellation « ex-RDA » n'a plus, aujourd'hui, de justification que dans une perspective particulière, celle de la géographie historique.

Depuis la fin du régime solidaire d'exception en 1994, qui avait soutenu le processus de transformation des économies est-allemandes, les " nouveaux » Länder partagent le lot des " anciens » : ils sont intégrés dans le même système général de la péréquation financière, le Finanzausgleich (voir infra). En l'espace de dix ans seulement après l'Unité, ils se sont largement différenciés les uns des autres, chacun présentant aujourd'hui un profil économique propre, à l'instar des Länder ouest-allemands.

Si les trois villes-Etats : Berlin, Hambourg et Brême présentent une économie à dominante tertiaire, les services s'y distinguent par leur nature. Berlin héberge de nombreuses administrations dont les fédérations professionnelles et les lobbies qui se sont installés à proximité du gouvernement fédéral et du Bundestag. Les sites portuaires Hambourg (premier port maritime d'Allemagne) et Brême (dont le port est situé dans la commune de Bremerhaven, une autre enclave à l'intérieur du Land de Basse-Saxe, mais rattachée administrativement au Land de Brême), se caractérisent par une prédominance du commerce et de la logistique. Si l'industrie n'y est pas absente, elle est directement liée aux activités portuaires, comme la construction navale ou l'agro-alimentaire; ces Etats hanséatiques sont également les deux principaux sites de production d'Airbus outre-Rhin. Quant à Bremerhaven, il est le premier site allemand des éoliennes offshore.

D'autres Länder, étendus mais à faible densité de population, offrent un profil économique plus disparate, avec un secteur primaire plus développé qu'ailleurs. C'est le cas notamment du Mecklembourg, plat pays bordé au nord par la Mer Baltique, où $30 \%$ de la population vit dans des communes de moins de 2000 habitants et dont les deux tiers de la surface d'exploitation sont voués à l'agriculture. Mais cette ruralité, propice aux activités touristiques (l'île de Rügen est l'une des plus réputées d'Allemagne), n'exclut pas les activités industrielles comme l'agroalimentaire et surtout la construction navale (Rostock), ni encore moins les services de lo- 
gistique : le Land est le fief allemand des centres d'appel et l'un des principaux centres de prestations postales sur le segment concurrentiel. Malgré son point fort qui est d'être une plateforme d'échanges avec l'espace baltique (via notamment la ville hanséatique de Greifswald), le Land n'en reste pas moins excentré, c'est-à-dire à l'écart des grands axes terrestres de communication transeuropéens, ce que reflète aussi son faible poids économique.

Le profil social des Länder en 2005 (données en milliers)

\begin{tabular}{|c|c|c|c|c|c|c|c|}
\hline & \multirow[t]{2}{*}{ Actifs } & \multirow[t]{2}{*}{$\begin{array}{l}\text {... dans } \\
\text { l'indus. }\end{array}$} & \multirow[t]{2}{*}{$\begin{array}{l}\text { Taux de } \\
\text { chômage }\end{array}$} & \multicolumn{2}{|c|}{$\begin{array}{l}\text { Salaire brut moyen/an } \\
\text { industrie }(e n €)\end{array}$} & \multirow[t]{2}{*}{ Etudiants } & \multirow[t]{2}{*}{ Apprentis } \\
\hline & & & & Ouvrier & Employé & & \\
\hline BW & 5093 & 1952 & $7,8 \%$ & 35767 & 54565 & 244 & 198 \\
\hline BS & 3401 & 957 & $13,0 \%$ & 33360 & 49981 & 152 & 146 \\
\hline BA & 5926 & 1899 & $8,9 \%$ & 34020 & 56510 & 252 & 256 \\
\hline B & 1434 & 235 & $21,5 \%$ & 34109 & 54203 & 133 & 55 \\
\hline BB & 1129 & 287 & $19,9 \%$ & 26720 & 41385 & 42 & 51 \\
\hline HB & 262 & 66 & $18,3 \%$ & 35656 & 53209 & 35 & 15 \\
\hline HН & 802 & 145 & $12,9 \%$ & 38498 & 58027 & 70 & 32 \\
\hline $\mathbf{H}$ & 2760 & 756 & $10,9 \%$ & 34157 & 52874 & 162 & 105 \\
\hline MP & 732 & 164 & $22,1 \%$ & 24809 & 37015 & 34 & 44 \\
\hline NRW & 7637 & 2275 & $13,2 \%$ & 34322 & 51799 & 477 & 312 \\
\hline $\mathbf{R P}$ & 1813 & 548 & $9,9 \%$ & 34916 & 53072 & 102 & 75 \\
\hline $\mathbf{S}$ & 435 & 124 & $11,7 \%$ & 36098 & 49102 & 20 & 22 \\
\hline SX & 1839 & 569 & $20,0 \%$ & 24826 & 38159 & 106 & 87 \\
\hline SA & 1035 & 283 & $21,7 \%$ & 25804 & 39449 & 51 & 54 \\
\hline SH & 1250 & 273 & $13,0 \%$ & 32579 & 50458 & 47 & 51 \\
\hline $\mathbf{T}$ & 1019 & 334 & $18,6 \%$ & 23877 & 37087 & 49 & 51 \\
\hline$R F A$ & 36566 & 10869 & $13,0 \%$ & 33435 & 52442 & 1977 & 1553 \\
\hline
\end{tabular}

Source des données : Statistisches Bundesamt, Bundesländer 2007. *) données administratives de l'Agence fédérale pour l'emploi.

La Basse-Saxe limitrophe à l'ouest, second Land par la superficie après la Bavière, présente en Allemagne l'une des structures économiques les plus diversifiées, ce qui n'est pas étranger à sa topographie structurée par un réseau hydrographique sud-nord-ouest (des monts du Hartz et du Weserbergland vers la Mer du Nord) entrecoupé de canaux transversaux est-ouest dont le Mittellandkanal, tronçon principal d'un réseau de canaux est-ouest qui relie le Rhin à la Weser (estuaire : Brême), à l'Elbe (axe Hambourg-Prague) et à l'Oder. Cet axe de communication fluvial, aussi important que les liaisons routières et ferroviaires Paris-Varsovie, passe comme celles-ci par Hanovre et Wolfsburg. Schématiquement, c'est dans la zone de ces infrastructures de communication que se situe l'essentiel de l'industrie, vouée principalement à l'automobile (VW à Wolfsburg et Hanovre), et aux TIC (Hanovre). C'est aussi à Hanovre que se trouve le premier site allemand de foires/expositions internationales (avant Francfort/Main). Au nord de cet axe se situent les plaines alluviales s'étendant jusqu'à la Mer du nord et la lande de Lunebourg, vouées à l'agriculture et à l'industrie agroalimentaire. La zone plus montagneuse au sud présente de fortes disparités : la vallée de la Leine (sud-nord) est à dominante agricole ; les marges occidentales de l'ancien Rideau de Fer sont à faible activité, sauf les monts du Hartz, région touristique. Deux pôles de hautes technologies et d'industrie s'y distinguent : la région de Brunswick à proximité du Mittellandkanal, classée la plus intensive en R\&D de l'UE (avionique et biotechnologies surtout) et qui abrite aussi l'une des plus anciennes universités 
techniques d'Allemagne (la Carolo Wilhelmina, créée en 1745). Et la ville de Salzgitter, le plus gros port fluvial de l'Allemagne du nord et $3^{\mathrm{e}}$ centre industriel de la Basse-Saxe où se concentrent industrie des métaux (sidérurgie, moteurs) et électronique automobile.

\section{Les Länder sont souverains quant à leur politique structurelle}

Plus déterminante pour la diversité des profils des Länder est la politique économique que mènent leurs gouvernements. Car l'organisation fédérale de l'Allemagne leur confère de larges pouvoirs. La réforme du fédéralisme politique d'août 2006 les accroît encore, mais sans modifier l'architecture de la répartition des pouvoirs entre les différents échelons territoriaux.

Institutionnellement, les 16 Länder sont les collectivités territoriales constitutives de la RFA. Ils sont dotés chacun d'une Constitution propre, d'un parlement (Landtag, appelé Bürgerschaft dans les trois villes-Etat) et d'un gouvernement : son chef, appelé ministre-président, est plus puissant qu'un ministre fédéral. Car ces Etats, souverains sur leur territoire, sont bien plus qu'une entité administrative 'déconcentrée' : ce sont de véritables Etats fédérés.

Dans la marche des affaires de la Fédération (Bund), ils ont un pouvoir de co-décision via le Bundesrat, la chambre du Parlement allemand où siègent les représentants de leurs gouvernements et sans l'aval de laquelle aucune loi touchant aux prérogatives des Länder ne peut être adoptée (c'est le cas d'un grand nombre de textes). Ce pouvoir constitue la première facette de ce fédéralisme coopératif qui caractérise l'Allemagne, avec sa répartition à la fois fonctionnelle et sectorielle des pouvoirs entre les membres de l'ensemble solidaire qu'est la République fédérale.

La seconde facette en est la répartition sectorielle des compétences législatives. Ainsi, les Länder sont seuls souverains en matière d'éducation, de culture et de médias. Dans la plupart des autres domaines, il y a concurrence entre les compétences du Bund et celles des Länder : ces derniers sont habilités à légiférer " aussi longtemps et pour autant que la Fédération n'a pas fait par une loi usage de sa compétence législative » (art. $72 \S 2$ de la Loi fondamentale). Or dans les faits, et malgré un catalogue très restreint de compétences exclusives, le Bund se révèle le législateur dominant, la compétence concurrente jouant en sa faveur en vertu de la primauté du droit fédéral (art. 31 de la Loi fondamentale). Au fil du temps, cette compétence concurrente avait fini par mener à une centralisation rampante des pouvoirs qui, en rognant la latitude des Länder sur leur territoire, était l'une des principales sources d'immobilisme de l'Allemagne et de nivellement des performances des Länder. Cette dérive a été en partie corrigée par la réforme du fédéralisme politique qui a clarifié la répartition des pouvoirs législatifs entre Bund et Länder. Elle a en outre réduit la part des lois fédérales soumises à approbation du Bundesrat, passée de $60 \%$ à un peu moins de $40 \%$ désormais.

La troisième facette du fédéralisme concerne la répartition fonctionnelle des pouvoirs. "Les Länder exécutent les lois fédérales à titre de compétence propre », stipule l'art. 83 de la Loi fondamentale. Autrement dit : ce sont les Länder qui exercent seuls le pouvoir administratif. Le Bund n'a en effet pas d'administration centrale, sauf dans les rares domaines qui relèvent de sa compétence régalienne exclusive : défense et affaires étrangères pour l'essentiel aujourd'hui. Les Länder mettent donc en œuvre les lois soit pour le compte du Bund (administration déléguée), soit au titre de leurs compétences propres. Dans d'autres cas encore, Bund et Länder coopèrent pour l'exécution de tâches communes, dont la liste a été nettement réduite lors de la réforme du fédéralisme politique. Le fait que les Länder disposent de compétences 
législatives somme toute résiduelles au regard du catalogue établi par la Constitution ne leur interdit donc nullement de mener leur propre politique de développement structurel. Bien au contraire, puisque ce sont eux qui disposent du pouvoir administratif, ce qui les habilite à gérer leurs affaires en pleine autonomie, que ce soit pour le compte de la Fédération, au titre des tâches communes, ou dans l'intérêt de leur site économique.

De plus, le Bund et les Länder étant « autonomes et indépendants les uns des autres dans leur gestion budgétaire » (art. $109 \S 1$ de la Loi fondamentale), les Länder sont souverains pour gérer la part des recettes fiscales qui leur revient après la péréquation financière à laquelle sont soumis Bund, Länder et communes (Finanzausgleich). Quelque $70 \%$ des recettes fiscales perçues outreRhin sont ainsi partagées entre les divers échelons territoriaux. Certes, les Länder n'ont qu'une faible autonomie pour définir une fiscalité originale, et peu d'impôts leur reviennent en propre (comme l'impôt sur les successions ou la taxe sur les véhicules). Mais ils sont pleinement souverains en ce qui concerne la gestion de leurs finances, n'étant soumis qu'au contrôle législatif de leur Landtag et réglementaire de leur Cour des comptes. Cette liberté leur confère la latitude requise pour mener la politique de développement régional définie par chacun.

Les budgets des Länder en 2005 (en millions $€$ )

\begin{tabular}{|lrrrrr|}
\hline & Recettes $^{\mathbf{a})}$ & Dépenses $^{\mathbf{b})}$... & $\begin{array}{c}\text {... dont } \\
\text { personnel... }\end{array}$ & $\begin{array}{c}\text {...et } \\
\text { investissement }\end{array}$ & Dette $^{\text {d) }}$ \\
Bade-Wurtemberg & 29604 & 45205 & 18988 & 1980 & 39541 \\
Basse-Saxe & 20450 & 31559 & 12263 & 1310 & 47855 \\
Bavière & 34783 & 51576 & 21177 & 2770 & 23077 \\
Berlin & 13814 & 21580 & 6642 & 2450 & 57380 \\
Brandebourg & 8377 & 11962 & 3570 & 930 & 16928 \\
Brême & 2383 & 4142 & 1279 & 660 & 12303 \\
Hambourg & 7155 & 10027 & 3372 & 850 & 21162 \\
Hesse & 17447 & 28094 & 10302 & 1100 & 30233 \\
Mecklembourg-P. & 5643 & 8493 & 2649 & 610 & 10777 \\
Rhénanie du N-W. & 49995 & 80748 & 30355 & 5650 & 108939 \\
Rhénanie-Palatinat & 10616 & 15859 & 6693 & 1010 & 24638 \\
Sarre & 2831 & 4428 & 1801 & 250 & 8102 \\
Saxe & 14177 & 19107 & 6153 & 1840 & 12086 \\
Saxe-Anhalt & 8294 & 12375 & 3807 & 1100 & 19223 \\
Schleswig-Holstein & 7270 & 11899 & 4673 & 490 & 20918 \\
Thuringe & 7676 & 10604 & 3550 & 1070 & 15052 \\
RFA & 240513 & 357692 & 137273 & - & 468214 \\
\hline
\end{tabular}

Source des données : Statistische Ämter des Bundes und der Länder (www.destatis.de), sauf pour les dépenses d'investissement (source : Sachverständigenrat, Staatsverschuldung wirksam begrenzen, mars 2007). a) Recettes fiscales (Länder et communes) perçues après les diverses péréquations financières, dont celle effectuée au titre du Fonds Deutsche Einheit; b) Länder et communes, sauf pour les villes-Etat Berlin, Brême et Hambourg ; $\mathrm{c}$ ) pour mémoire : les dépenses d'investissement du Bund s'élèvent à 22,86 milliards $€ ; d$ ) Länder et communes, sauf pour les villes-Etat Berlin, Brême et Hambourg; sans les dettes contractées entre 22,86 milliards $€$; d) Länder et comm
les diverses collectivités territoriales.

Elle présente toutefois un inconvénient majeur dont on a pris pleinement la mesure voici une dizaine d'années seulement, au moment de la définition des règles liées à l'adoption de la monnaie unique. En effet, les critères relatifs à la dette dans le Pacte de stabilité et de croissance ne sont contraignants que pour le Bund. Or celui-ci ne maîtrise que moins de la moitié des dépenses et engagements (les Länder réalisent quelque $40 \%$ des dépenses publiques allemandes). Au plan intérieur, il n'existe pas d'équivalent allemand au Traité de Maastricht 
(seule s'applique la législation adoptée par la plupart des Länder sur le modèle de l'art. 115 de la Loi fondamentale qui interdit un recours au déficit d'un montant supérieur à celui des investissements). Cette absence de contrainte pesant sur les dépenses a abouti au fait que presque tous les Länder sont lourdement endettés, sauf trois, au budget aujourd'hui non seulement équilibré, mais aussi sans nouvel endettement à brève échéance : la Bavière, la Saxe et, depuis 2006, le Mecklembourg.

Plus de la moitié de la dette allemande reste imputable aux Länder, ce qui a forcé le lancement de la réforme du fédéralisme financier en débat depuis le début de l'année 2007. Et incité récemment quatre Länder à engager une politique de rigueur pour présenter un budget équilibré en 2011 : le Bade-Wurtemberg, la Basse-Saxe, le Brandebourg et la SaxeAnhalt. D'autres, comme Hambourg, ont commencé à réduire drastiquement leurs dépenses.

Ce laxisme budgétaire se nourrit par ailleurs d'une dérive qu'a connue le fédéralisme financier au fil des décennies. L'objectif du partage des ressources consiste en théorie à assurer des conditions de vie équivalentes (gleichwertige Lebensverhältnisse; art. $72 \S 2$ de la Loi fondamentale) sur l'ensemble du territoire de la RFA, c'est-à-dire à garantir l'équité des chances entre les 16 Länder - et non pas l'égalité, ainsi qu'a dû le rappeler le Tribunal constitutionnel fédéral dans un arrêt rendu en 1999 et dans lequel il établit le principe constitutionnel de «l'interdiction du nivellement » (Verbot der Nivellierung).

Capacité financière des Länder avantlaprès le Länderfinanzausgleich - 2005 (en millions $€$ )

\begin{tabular}{|lrrr|}
\hline & Avant & Après & Part versée/perçue \\
Bade-Wurtemberg & 28202 & 25993 & -2209 \\
Basse-Saxe & 17860 & 18220 & +359 \\
Bavière & 32279 & 30060 & -2219 \\
Berlin & 7138 & 9579 & +2441 \\
Brandebourg & 5079 & 5660 & +581 \\
Brême & 1543 & 1909 & +366 \\
Hambourg & 6027 & 5650 & -377 \\
Hesse & 16457 & 14864 & -1593 \\
Mecklembourg-P. & 3358 & 3786 & +428 \\
Rhénanie du N-W. & 42844 & 42357 & -487 \\
Rhénanie-Palatinat & 8879 & 9171 & +292 \\
Sarre & 2249 & 2361 & +112 \\
Saxe & 8385 & 9392 & +1007 \\
Saxe-Anhalt & 4889 & 5469 & +580 \\
Schleswig-Holstein & 6280 & 6425 & +145 \\
Thuringe & 4561 & 5134 & +573 \\
RFA & 196029 & 196029 & 6885 \\
\hline
\end{tabular}

Source des données: Bundesministerium der Finanzen, Bund-Länder Finanzbeziehungen auf der Grundlage der geltenden Finanzverfassungsordnung, Berlin, octobre 2006 (www.bundesfinanzministerium.de).

Le problème ne se situe pas tant au plan de la répartition verticale du montant global des ressources fiscales allemandes entre les échelons territoriaux, dans le cadre de laquelle la quotepart de chaque Land lui est attribuée au prorata de son nombre d'habitants. Il se situe au niveau horizontal, dans le mécanisme de solidarité jouant entre des Länder de poids disparate et qui vise à atténuer les inégalités de richesse : le Länderfinanzausgleich. Dans ce cadre, une partie des recettes fiscales revenant aux Länder 'les plus riches' (donateurs) est redistribuée aux 
'moins riches' (bénéficiaires) afin d'assurer à chacun une capacité financière s'élevant à au moins $95 \%$ de la moyenne. Le total ainsi redistribué s'élevait à près de 7 milliards $€$ en 2005.

Si ce mécanisme a bénéficié à une Bavière sous-développée au sortir de la guerre et si, conjugué au Pacte de Solidarité de l'Aufbau Ost de même qu'aux fonds structurels européens, il profite à des économies dynamiques comme la Saxe ou la Thuringe, il s'est révélé contreproductif pour la compétitivité de Länder comme Berlin (Bourgeois, 2006), mais aussi Brême, de loin le plus endetté de tous avec une dette de près de $19000 €$ par tête, ou la Sarre $(9600 €)$.

Plus généralement, il tasse la croissance en réduisant la compétition entre sites : les cinq donateurs (Bade-Wurtemberg, Bavière, Hambourg, Hesse et Rhénanie du Nord-Westphalie) se voient privés d'une partie des fruits de leur politique; les récipiendaires (tous les autres) ne sont pas réellement incités à l'efficience. Selon les experts, le Länderfinanzausgleich en l'état actuel coûterait près d'un point de croissance à l'Allemagne (Gabel, 2001). Il s'agit dorénavant de trouver un nouvel équilibre afin de redynamiser la concurrence entre sites tout en préservant la nature solidaire du fédéralisme financier ; le principe lui-même n'est pas en cause.

\section{Des politiques de compétitivité et de cohésion territoriale très diverses}

L'objectif d'équivalence, corollaire de la cohésion territoriale, s'applique pareillement aux régions à l'intérieur de chaque Land. Sur son territoire, chaque gouvernement mène donc la politique qu'il juge utile pour développer la croissance et la stabilité de son économie, la prospérité des citoyens, des entreprises et des régions. Cela inclut politique industrielle, énergétique, éducative, technologique, culturelle, aménagement du territoire et création d'infrastructures, programmes d'aides à la création d'entreprises ou de développement structurel régional. La Bavière, par exemple, consacre quelque $12 \%$ de son budget (36 milliards $€$ en 2007) au seul soutien aux PME, aux infrastructures et à l'innovation. Autonome dans ses domaines de souveraineté, chaque Land l'est également dans la mise en œuvre de la politique fédérale ou des missions communes. Tout l'art consiste dès lors pour chaque gouvernement à y intégrer intelligemment ses propres orientations et priorités. C'est là un facteur primordial pour l'identité territoriale.

Le gouvernement d'un Land a donc la possibilité de mener sa propre politique économique - au sens allemand du terme. Son rôle, tout comme d'ailleurs celui du gouvernement fédéral, consiste en effet essentiellement à créer et entretenir un cadre favorable aux activités : à l'émergence d'initiatives, à l'investissement, à la création d'entreprises et à l'emploi, sans oublier le développement de la qualité de vie. Et un gouvernement ne peut définir ces orientations générales pour son Land qu'en s'appuyant sur d'autres acteurs autonomes : communes, chambres de commerce et d'industrie, chambre des métiers, universités, fédérations, entreprises, etc. Car, selon le principe de subsidiarité, ce sont eux les véritables acteurs économiques sans qui aucune politique n'est possible à l'échelon supérieur.

L'effort politique principal d'un gouvernement en matière de développement de son site économique se fondera donc sur une approche de bottom up propice à l'émergence des initiatives des acteurs privés locaux et visera à en impulser la coordination à l'échelle du Land, tout en mettant en œuvre les axes de la politique fédérale qu'il a lui-même en partie contribué à définir. C'est cet ensemble d'implications à divers échelons, d'impulsions actives, de coordination des moyens et des initiatives qu'on désigne communément par le terme de Standortpolitik. 
Budgets des Länder ") - ventilation des principales dépenses 2003 (en milliards $€$ )

\begin{tabular}{|lccccccccc|}
\hline & $\begin{array}{c}\text { Gouvernement/ } \\
\text { administration }\end{array}$ & Sécurité & $\begin{array}{c}\text { Formation/ } \\
\text { Enseignemt. }\end{array}$ & $\begin{array}{c}\text { Culte et } \\
\text { culture }\end{array}$ & $\begin{array}{c}\text { Protect. } \\
\text { sociale }\end{array}$ & $\begin{array}{c}\text { Sport/ } \\
\text { loisirs }\end{array}$ & Urbanisme & $\begin{array}{c}\text { Soutien à } \\
\text { l'économie }\end{array}$ & $\begin{array}{c}\text { Transports et } \\
\text { communications }\end{array}$ \\
Bade-Wurtembg. & 2,35 & 4,04 & 9,70 & 1,11 & 6,40 & 1,82 & 3,09 & 1,51 & 1,76 \\
Basse-Saxe & 1,68 & 3,41 & 6,86 & 0,51 & 5,86 & 1,14 & 1,43 & 1,19 & 1,18 \\
Bavière & 2,75 & 5,31 & 10,96 & 1,32 & 8,03 & 1,91 & 3,47 & 2,27 & 2,25 \\
Berlin & 0,83 & 2,42 & 3,60 & 0,58 & 4,79 & 0,65 & 1,71 & 0,23 & 0,01 \\
Brandebourg & 0,77 & 1,40 & 1,86 & 0,23 & 1,89 & 0,41 & 0,93 & 0,82 & 0,48 \\
Brême & 0,21 & 0,37 & 0,68 & 0,09 & 0,86 & 0,12 & 0,12 & 0,29 & 0,07 \\
Hambourg & 0,53 & 1,15 & 1,83 & 0,23 & 2,00 & 0,30 & 0,20 & 0,13 & 0,35 \\
Hesse & 1,61 & 2,88 & 5,08 & 0,56 & 5,23 & 1,01 & 1,69 & 0,85 & 0,93 \\
Mecklembourg-P. & 0,57 & 0,93 & 1,61 & 0,17 & 1,28 & 0,35 & 0,45 & 0,74 & 0,33 \\
Rhénanie N.-W. & 4,15 & 8,22 & 16,53 & 1,39 & 15,52 & 2,45 & 5,27 & 2,18 & 1,98 \\
Rénanie-P. & 0,88 & 1,70 & 3,22 & 0,30 & 2,77 & 0,53 & 0,69 & 0,67 & 0,69 \\
Sarre & 0,28 & 0,46 & 0,83 & 0,06 & 0,84 & 0,13 & 0,22 & 0,16 & 0,09 \\
Saxe & 1,15 & 1,89 & 3,80 & 0,69 & 2,82 & 0,71 & 1,11 & 0,98 & 0,64 \\
Saxe-Anhalt & 0,74 & 1,31 & 2,32 & 0,31 & 1,98 & 0,52 & 0,63 & 0,95 & 0,38 \\
Schleswig-H. & 0,69 & 1,23 & 2,21 & 0,20 & 2,20 & 0,38 & 0,63 & 0,37 & 0,36 \\
Thuringe & 0,70 & 1,10 & 2,21 & 0,30 & 1,53 & 0,40 & 0,56 & 0,74 & 0,67 \\
Bund & 4,33 & 4,84 & 3,90 & 0,36 & 502,10 & 2,24 & 1,25 & 20,30 & 10,09 \\
$\boldsymbol{\Sigma}$ RFA & 24,24 & 42,64 & 77,12 & 8,35 & 566,10 & 15,01 & 23,44 & 34,40 & 22,26 \\
\hline
\end{tabular}

Source des données : Destatis, Statistisches Jahrbuch 2006; chiffres arrondis par nos soins. *) Länder et communes. NB: Dernières donnees disponibles : 2003. L'actualisation des données collectées par l'Office fédéral des Statistiques est un exercice de longue haleine dans la mesure où l'autonomie budgétaire des Länder inclut une certaine liberté dans la définition des postes au sein des grands chapitres de dépenses. 
Il revient donc à chaque Land de donner corps aux grands objectifs d'équilibre économique communs à l'ensemble de l'Allemagne comme aux siens propres. L'originalité des choix faits par chaque Land sera bien sûr fonction des orientations idéologiques de la majorité élue, de chaque gouvernement en place. La Standortpolitik est donc aussi le moyen pour chacun de cultiver sa singularité et de se distinguer dans cette course aux résultats que se livrent les Länder dans un pays où, fondamentalement, la concurrence est considérée comme une saine émulation, qu'il s'agisse d'entreprises ou de territoires. La concurrence entre les Länder se révèle par là même aussi un des facteurs-clefs de la cohésion nationale, puisqu'elle promeut la confrontation des intérêts, préalable aux négociations nécessaires à la prise de décisions conjointes.

Or dans l'approche allemande du système économique, pour que la concurrence puisse pleinement produire ses effets positifs sur la collectivité, elle doit être régulée. Cette orientation ordo-libérale est largement partagée par toutes les forces politiques. La diversité des choix effectués par les Länder concourt ainsi au processus permanent de réflexion et d'ajustement national. La cohésion collective est en outre assise sur le principe de l'auto-régulation: dans cette concurrence entre sites, les parties prenantes ont en effet obligation de respecter un comportement visant à préserver l'unité fédérale (bundesfreundliches Verhalten). Ce principe, qui a force constitutionnelle, répond au souci institutionnalisé de garantir l'intérêt général dans le respect de la singularité des intérêts régionaux.

La marge de manœuvre des Länder est donc très grande. Et c'est du dynamisme de chaque Land, entretenu par la compétition, que dépend la prospérité collective du site Allemagne. Qu'un des 'poids lourds' voie faiblir son économie, et c'est l'Allemagne qui se trouve sousperformante. A l'inverse, la bonne tenue des affaires dans un Land accroît la prospérité de tous. C'est pour cela aussi que les Länder se livrent à un benchmarking permanent afin de déceler leurs forces et faiblesses et d'y apporter les réponses politiques requises.

Systèmes de formation : le palmarès des Länder (2005)

\begin{tabular}{|c|c|c|c|c|c|c|}
\hline & \multicolumn{3}{|c|}{ Taux de performance de la formation (en \%) } & \multicolumn{3}{|c|}{ Coût par tête de la formation (en $€$ ) } \\
\hline $\boldsymbol{B} \boldsymbol{W}$ & 59,2 & 68,2 & 54,9 & 4900 & 4000 & 8570 \\
\hline$B S$ & 46,0 & 48,2 & 47,8 & 4800 & 3400 & 9110 \\
\hline $\boldsymbol{B} \boldsymbol{A}$ & 66,5 & 64,7 & 51,8 & 5100 & 3200 & 8340 \\
\hline $\boldsymbol{B}$ & 39,4 & 31,1 & 49,2 & 5700 & 3300 & 7530 \\
\hline$B B$ & 52,3 & 46,4 & 41,7 & 4400 & 2300 & 6020 \\
\hline$H B$ & 28,1 & 41,3 & 54,7 & 5200 & 3300 & 5940 \\
\hline $\boldsymbol{H H}$ & 46,5 & 58,7 & 46,5 & 6600 & 4400 & 6430 \\
\hline $\boldsymbol{H}$ & 40,6 & 51,7 & 40,6 & 4700 & 3400 & 5870 \\
\hline$M P$ & 49,9 & 41,4 & 54,1 & 4400 & 2400 & 8580 \\
\hline$N R W$ & 49,0 & 42,6 & 39,5 & 4800 & 3200 & 6950 \\
\hline $\boldsymbol{R P}$ & 47,3 & 47,3 & 42,8 & 4600 & 3200 & 6180 \\
\hline$S$ & 41,4 & 47,2 & 48,3 & 4300 & 3300 & 8610 \\
\hline$S X$ & 61,0 & 52,6 & 52,6 & 4800 & 2700 & 7850 \\
\hline$S A$ & 37,0 & 44,5 & 42,9 & 5100 & 2800 & 8870 \\
\hline$S H$ & 55,8 & 51,2 & 47,6 & 4700 & 3400 & 7390 \\
\hline$T$ & 51,9 & 47,6 & 47,7 & 5700 & 3200 & 8340 \\
\hline Moyenne & 50,8 & 53,5 & 45,8 & 4900 & 3300 & 7510 \\
\hline
\end{tabular}

Sources : Taux de performance : A. PLÜNNECKE, O. STETTES, «Bildung in Deutschland. Ein Benchmarking der Bundesländer aus bildungsökonomischer Perspektive », IW-Analysen, $n^{\circ}$ 10/2005. Coût de la formation : Destatis/IWD, $n^{\circ} 20 / 05$. 
Le benchmarking auquel ils se livrent pour évaluer leurs systèmes de formation en est un des nombreux exemples. Cette évaluation est plus cruciale que jamais dans le contexte de la réforme en débat qui s'oriente vers une concurrence accrue des sites d'éducation et de recherche. L'objectif en est un regain de compétitivité dans l'économie du savoir.

Les politiques de compétitivité menées par les Länder sont donc diverses, construites sur des structures et des cultures régionales distinctes, bien sûr, mais également déterminées par les choix des électeurs et l'orientation idéologique de chaque gouvernement. La continuité politique est dès lors un facteur décisif pour l'évolution de l'économie d'un Land. Trois cas sont particulièrement révélateurs à cet égard : la Bavière (CSU ; majorité absolue depuis 1962), le Bade-Wurtemberg (où le CDU est au gouvernement depuis 1952, en coalition tantôt avec le SPD ou le FDP) et la Rhénanie du Nord-Westphalie (fief du SPD, dans des coalitions diverses, notamment avec les Verts, jusqu'en 2005).

Etat libre de Bavière : lente et systématique construction de la compétitivité. Le ministreprésident Edmund Stoiber se place, comme ses prédécesseurs, dans le prolongement de l'action d'un Franz Josef Strauss qui fut à la tête du gouvernement bavarois de 1978 à 1988. Ce dernier, en qualité de ministre fédéral des Finances sous la première grande coalition (1966-69), co-rédigea avec son homologue de l'Economie, Karl Schiller (SPD), la Loi de promotion de la stabilité et de la croissance (1967) qui guide toujours l'action de tout gouvernement allemand.

Cette continuité a permis de transformer en 40 ans un Land rural et déshérité, sans ressources énergétiques notables, en un site industriel high-tech, ce que traduit la devise adoptée dans les années 1990 : "l'alliance entre l'ordinateur portable et la culotte de peau ». Cette transformation, favorisée par une structure administrative centralisée (cas unique en Allemagne), s'est effectuée en cinq étapes successives, mais aux effets cumulatifs : l'accueil massif de réfugiés allemands issus d'Europe centrale (années 1950), une politique de localisations industrielles avec pour point de cristallisation l'installation à Munich du siège de Siemens qui a eu pour effet, par grappage, le développement de clusters industriels (années 1960), l'adoption d'une Loi sur le soutien aux PME (Mittelstandsförderungsgesetz) qui servira de modèle aux programmes de soutien adoptés par les autres Länder et le Bund (années 1970), une politique conséquente de développement de la R\&D et des nouvelles technologies, notamment les biotechnologies (années 1980), suivie d'une réorientation ciblée vers l'innovation high-tech (années 1990).

A cela se sont ajoutées une politique systématique de désenclavement et une politique énergétique centrée sur le nucléaire (quelque $70 \%$ de l'approvisionnement du Land) et l'hydroélectrique (la Bavière fournit près de la moitié de l'approvisionnement allemand). L'effort intense d'investissement, possible grâce au Finanzausgleich, s'est accompagné d'un effort particulier consenti aux ressources humaines : le baccalauréat bavarois est le plus prisé d'Allemagne, les universités cumulent les labels «élite » et « excellence», les salariés figurent parmi les mieux qualifiés et le taux de chômage est l'un des plus faibles (7,8 \% en 2006).

Bade-Wurtemberg : une culture industrieuse décuplée par la mise en réseau des compétences. Ce Land, de création tardive, a dû construire son identité, contrairement à la Bavière millénaire. Il l'a fait en misant sur la tradition de créativité et d'innovation de ses habitants (Karl Benz, Gottlieb Daimler, Robert Bosch pour ne citer que les plus célèbres de ses inventeurs historiques) comme sur ses atouts en termes de qualité de vie : les agglomérations et pôles d'activité sont certes denses, disséminés sur l'ensemble du territoire, mais sans nuire au caractère naturel de l'environnement. Aujourd'hui, l'effort de R\&D représente $4 \%$ du PIB du Land (voire $7 \%$ dans la région de Stuttgart). Si le Land héberge de grands groupes comme Carl Zeiss, il est le fief par excellence des PME, dont nombre de ces champions mondialement réputés comme Stihl ou Kärcher.

La politique structurelle de ce Land, dont la seule ressource naturelle notable est le capital humain, est discrète par nature et conforme à la réputation de 'fourmis industrieuses' de ses habi- 
tants. Elle suit deux axes. D'une part la promotion de cette culture créative grâce à un intense effort de formation professionnelle (ses habitants sont les mieux qualifiés d'Allemagne : un habitant sur dix est diplômé de l'enseignement supérieur; le taux de chômage est le plus faible avec 7 \% seulement en 2006) et de transfert des technologies et savoirs essentiellement vers les PME (c'est dans ce Land que s'est créée la Fondation Steinbeis, acteur majeur mais pas unique de la diffusion de l'intelligence économique). D'autre part, l'aménagement d'un cadre favorable à ces pôles de compétitivité qui se sont constitués spontanément comme un écosystème au fil du temps autour des piliers de l'industrie locale : automobile, construction mécanique, biotechnologies et chimie.

La politique structurelle des gouvernements se place donc délibérément au service des acteurs économiques. Il est vrai que le Land se présente aujourd'hui comme un pôle de compétitivité pratiquement d'un seul tenant. Dès lors, sa politique de compétitivité se résume à cette formule de son ministre de l'Economie : "l'innovation naît dans un processus où l'économie, la science, la formation et la société agissent en réseau, et dont le centre est l'individu en tant que porteur des savoirs ». Si le Bade-Wurtemberg est bien armé pour la société du savoir, il doit, tout comme la Bavière, miser plus désormais sur l'avenir.

Rhénanie du Nord-Westphalie : reconversion grâce à une révolution technologique. Si ce Land structuré par le Rhin et la Ruhr, très divers du fait des provinces qu'il rassemble (la Rhénanie est catholique, la Ruhr protestante et la Westphalie mixte) n'est plus le «pays noir » qu'il fut jusque dans les années 1970, et si son industrie est aujourd'hui tertiarisée à un haut degré, il le doit à une politique de reconversion originale : du charbon aux TIC via le show biz. Berceau historique de la cogestion au cœur du «modèle rhénan » et théâtre en 1959 du Congrès de Bad Godesberg où fut définie la social-démocratie, sa tradition SPD a contribué à une transition en douceur, alors même que l'emploi industriel (et minier) chutait brutalement. En contrepartie, le Land (qui hébergeait la capitale allemande d'avant l'Unité : Bonn) a cherché à développer ses forces et son potentiel : la forte concentration de médias, la présence de l'opérateur historique des télécommunications, celle de la Deutsche Bahn se conjuguent à un réseau d'infrastructures de communication d'une densité rare, et ce dans un Land qui occupe une position centrale dans l'espace économique européen.

Trois temps se distinguent dans sa politique structurelle : jusqu'à la fin du $\mathrm{XX}^{\mathrm{e}}$ siècle, il s'agissait de compenser les déficits structurels par un soutien aux activités en déclin (notamment dans la Ruhr) et le développement de secteurs nouveaux (TIC); ensuite, il a fallu entretenir et soutenir le potentiel d'innovation existant : au soutien sectoriel ou territorial (la ville de Gelsenkirchen dans la Ruhr a un taux de chômage de quelque 17 \%) a donc succédé le soutien ciblé des pôles à fort potentiel. Mais cette politique de mise en réseau et de grappage restait pilotée par les pouvoirs publics et, surtout souffrait d'un financement trop indifférencié, menant à une mauvaise allocation des ressources alors même que le Land est lourdement endetté. Le changement de gouvernement en 2005 (arrivée au pouvoir d'une coalition CDU/FDP) a enclenché un recentrage plus systématique sur les pôles d'excellence, misant résolument sur les synergies public-privé et acceptant désormais l'existence de fortes disparités territoriales.

La reconversion du Land n'est pas achevée. Reste aussi à remédier au point faible du site qui se traduit par un taux de chômage de $12 \%$ en 2006 : la faible performance globale de son système éducatif - et universitaire. Seule émerge la RTWH d'Aix-la-Chapelle, au cour de l'Euregio Meuse/Rhin où se trouve également le centre de recherche de Jülich.

L'originalité des politiques structurelles que permet l'indépendance d'action des Länder explique aussi comment la Saxe, Land est-allemand, est devenue un de ces "paysages fleuris » qu'invoquait le chancelier Kohl lors de l'unification. Certes, elle ne 'pèse' que l'équivalent de Berlin ou Hambourg dans le PIB allemand, mais son potentiel est loin d'être épuisé. Surtout, cet Etat libre doit sa compétitivité au fait que, dès 1994, le ministre-président d'alors, Kurt Biedenkopf (CDU) rompt radicalement avec la politique globale et indifférenciée de 
soutien à la transformation qu'est l'Aufbau Ost, en ayant décelé l'un des premiers les effets pernicieux sur la compétitivité. Elle ne prenait en effet pas assez en compte les spécificités régionales. Sa réorientation est en débat dans le cadre de la réforme du fédéralisme financier et de la réduction des aides européennes dans le budget 2007/13 à la suite de l'élargissement de l'UE.

La Saxe, challenger de la Bavière. La Saxe dispose, tout comme le Bade-Wurtemberg, d'une longue culture industrieuse que le régime de la RDA avait essayé de maintenir, mais avec des résultats mitigés. Après 1990, c'est sur ces fondations et la présence d'un dense tissu de PME que le Land établit sa politique de transition/restructuration, menant de front modernisation des activités industrielles et stratégie d'innovation. C'est ainsi que le Land est (re)devenu un grand site automobile (des Trabant aux Passat), a (re)dynamisé sa construction mécanique et qu'il arbore fièrement le qualificatif de « Silicon Saxony » : Dresde est aujourd'hui le premier cluster européen de microélectronique (la ville accueillait, du temps de la RDA, le combinat Robotron).

Ce (re)positionnement s'est effectué d'une part grâce à une politique budgétaire rigoureuse qui a porté ses fruits : depuis 2006, le Land présente un budget équilibré. D'autre part, grâce à une priorité absolue accordée à l'investissement productif pour créer les conditions permettant de générer et dynamiser l'activité.

Cette politique est à l'opposé de celle pratiquée par les autres nouveaux Länder qui, le plus souvent, utilisent les fonds de l'Aufbau Ost et les aides européennes pour combler leurs trous budgétaires - une pratique abusive qui alimente les débats, posant la question du bien fondé d'une politique de cohésion qui repose trop exclusivement sur une logique de redistribution indifférenciée.

La forte internationalisation de l'économie saxonne (près de la moitié des exportations des nouveaux Länder lui est imputable) qui tire sa croissance est le résultat de cette politique originale, inscrite elle aussi dans la continuité. Le fait que le Land soit aujourd'hui gouverné par une grande coalition CDU/SPD n'en a pas modifié l'approche. Sur ce fond, l'élargissement de l'UE laisse aussi présager un partage des tâches affiné entre une production orientale plutôt low-tech pour l'instant et le high-tech saxon. Reste à réduire le chômage (le taux dépasse encore $18 \%$ en 2006) et, surtout, à enrayer le vieillissement démographique accentué par l'exode des jeunes grâce notamment à une politique de qualifications plus en accord avec le potentiel de compétitivité du Land.

\section{Au sein des Länder, les disparités régionales sont fortes}

Si les Länder présentent une grande diversité, au sein même de chacun d'entre eux existent de fortes disparités. Le Brandebourg qui entoure Berlin, par exemple, ne comprend qu'une seule région dynamique : la ceinture du Land capitale, avec une concentration d'activités high-tech dans l'agglomération de Potsdam. La région de Francfort-sur-Oder à la frontière polonaise souffre des conséquences d'une politique de localisations industrielles trop volontariste et généreusement subventionnée qui avait cherché à recréer, sur les ruines du combinat sidérurgique d'Eisenhüttenstadt qui produisait la moitié de l'acier de la RDA, des activités sectorielles identiques (Usinor, Motorola) - en négligeant le fait que ces pôles y avaient été érigés ex nihilo par le régime de RDA, c'est-à-dire sans pouvoir s'intégrer dans un tissu d'activités préexistant. Quant aux activités dans le reste de ce Land faiblement peuplé, elles se partagent entre agriculture et tourisme, surtout dans les marges proches des régions à faible activité du sud du Mecklembourg et du nord-est de la Saxe-Anhalt.

Si ces disparités sont en partie liées à la restructuration des économies est-allemandes après l'Unité et si elles y sont de ce fait plus prononcées qu'à l'ouest, elles sont loin d'être la spécificité 
des nouveaux Länder. Le nord-est rural de la Hesse, par exemple, qui avait été coupé de la Thuringe par le Rideau de Fer, fait partie de ces régions accusant un retard économique, au même titre que le nord du Schleswig-Holstein à la frontière danoise ou le nord-est de la Bavière à la frontière tchèque. Ces régions n'ont jamais franchi le pas vers des activités dynamiques, d'autant qu'elles sont loin des grands axes de communication allemands et intra-européens.

La question des infrastructures est en effet cruciale pour le développement économique local ou régional, comme le révèle la tendance à l'intégration de pôles d'activités distants entre eux, mais situés à proximité des grands axes. L'exemple-type en est celui des régions de Stuttgart et du Rhin-Main qui se densifient, préfigurant une tendance à la conurbation et à la concentration des activités comparable à celle de la Ruhr. C'est le cas aussi des agglomérations de Dresde, Potsdam et Iéna, en plein essor grâce à leur situation sur les axes Berlin-Hanovre et Dresde-Iéna-Erfurt. La structure établie des activités joue elle aussi un rôle fondamental dans ces évolutions : ce sont surtout les régions où domine une industrie low-tech qui présentent aujourd'hui une faible compétitivité. Ces régions en déclin se concentrent au nord du Main, et à l'ouest.

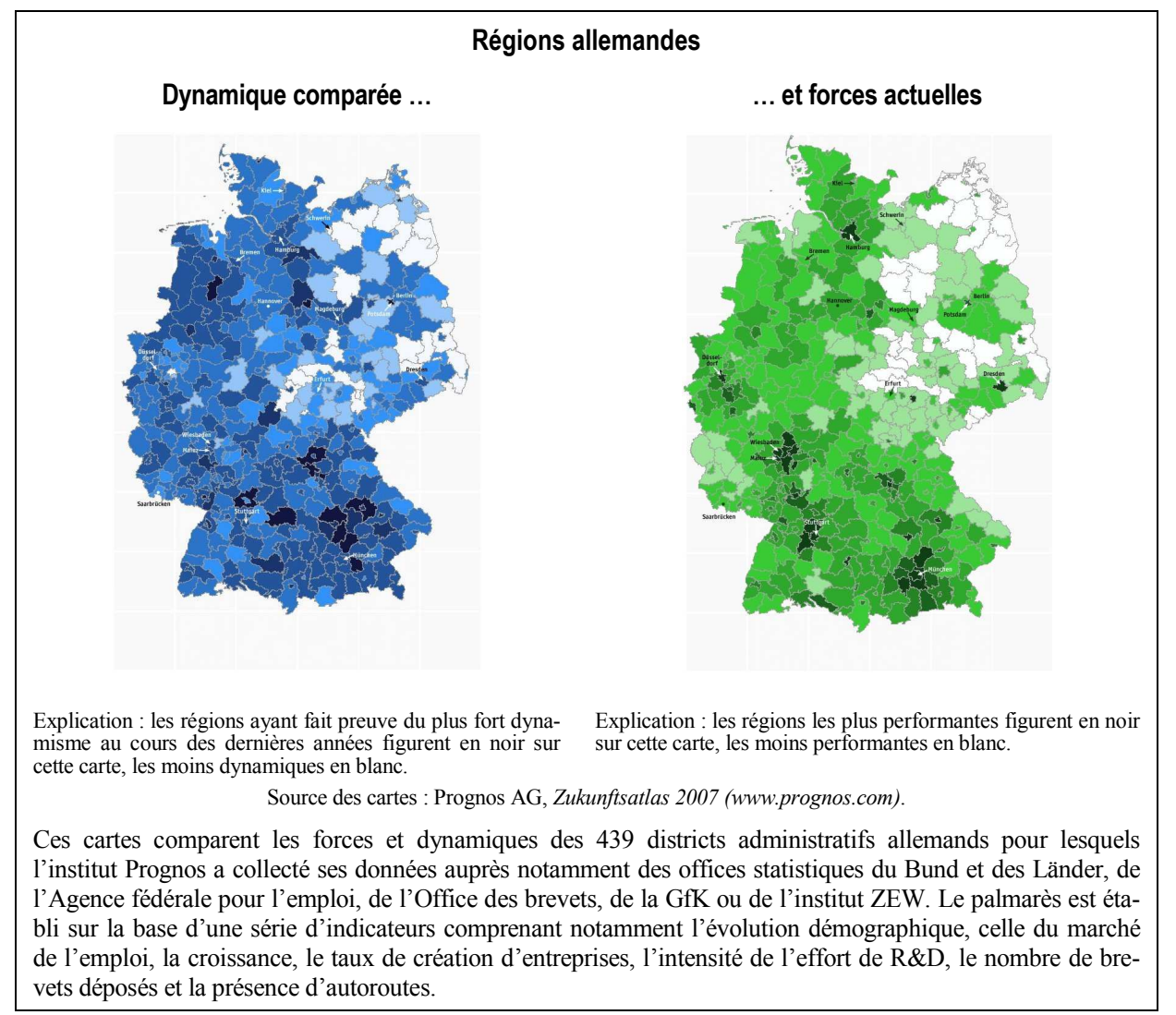

C'est ainsi que se dessine une Allemagne à « quatre vitesses » : l'ensemble sud et sud-ouest est de loin le plus compétitif; les performances du nord sont moyennes ; à l'est, quelques pôles au fort dynamisme se profilent dans un ensemble plutôt morose. 
Dans une Allemagne RedeVenue Compétitive, la Vitalité des LÄNDER revêt une importance décisive pour asseoir la croissance sur des bases durables. Une partie de leurs politiques de compétitivité se concentre sur les clusters qui bénéficient maintenant d'aides plus ciblées. Mais l'innovation technologique ne suffit pas. Le cadre des activités doit devenir encore plus incitatif: la plupart des Länder a engagé une modernisation de l'administration dans le sens d'une plus grande efficience et d'une proximité accrue avec les entreprises. Dans l'économie du savoir, la qualification des ressources humaines est un enjeu stratégique, et les Etats allemands ont commencé à réformer leurs systèmes de formation.

Mais leur latitude d'action dans un environnement global en mutation se heurte aujourd'hui aux limites de leurs compétences législatives et budgétaires. Car la centralisation rampante des pouvoirs aux mains du Bund et le co-pilotage de plus en plus caractérisé par la Commission européenne les privent de la souplesse requise pour adapter chacun le cadre réglementaire commun aux besoins spécifiques de sa propre économie. De ce fait, ils rendent la politique du Land peu lisible aux yeux de ses citoyens, ce qui contribue à nourrir leur méfiance envers le monde politique. Quant à la solidarité financière qui lie les Länder, elle inhibe leur croissance en bridant la dynamique propre à chacun. Seule une réforme en profondeur des règles du jeu du fédéralisme politique et financier permettra de redynamiser la concurrence entre des sites exposés à la mobilité des facteurs de production au sein de la RFA, mais sans la possibilité d'y apporter la réponse adéquate. Cette réforme, en cours de discussion, implique l'acceptation des disparités territoriales, corollaire d'un retour au dogme originel au fondement du fédéralisme allemand comme de son ordre économique et social : le principe d'équité des chances.

\section{Indications bibliographiques}

BERTELSMANN-STIFTUNG, Die Bundesländer im Standortwettbewerb 2005, Gütersloh, 2006

Bourgeols I., "Pas d'exception budgétaire pour Berlin », in Regards sur l'économie allemande, $\mathrm{n}^{\circ} 79$, décembre 2006

BOURGEOIS I., « 10 ans après l'unification : l'économie des nouveaux Länder en voie de normalisation », in BOURGEOIS I. (dir.), Allemagne 2001. Regards sur une économie en mutation, Cirac, Levallois-Perret, 2001

BUNDESMINISTERIUM DER FINANZEN, Bund-Länder Finanzbeziehungen auf der Grundlage der geltenden Finanzverfassungsordnung, Berlin, octobre 2006 (www.bundesfinanzministerium.de)

CERNAVIN O. et al., Cluster und Wettbewerbsfähigkeit von Regionen, Berlin, 2005

DESTATIS, Statistisches Jahrbuch 2006 (www.destatis.de)

DESTATIS, WZB, ZUMA, Datenreport 2006, Bonn, 2006

Fuest C., KerVian D. et Welter P., Wirtschaftliche Freiheit in den deutschen Bundesländern, FriedrichNaumann-Stiftung, novembre 2007 (www.fnst.org)

GABEL M., « La réforme du fédéralisme financier allemand. Beaucoup de bruit pour rien ? », in Regards sur l'économie allemande, $\mathrm{n}^{\circ} 52-53$, octobre 2001

HyPOVEREINSBANK, Fit für die Zukunft? Die Bundesländer im Vergleich, avril 2007 (www.hvb.de)

IW CONSUlT, Viertes Bundesländerranking, Cologne, septembre 2006 (www.iwconsult.de)

MC KINSEY, Projektbericht Perspektive-Deutschland 2005/06, Düsseldorf, 2006

PLÜNNECKE A., STETTES O., «Bildung in Deutschland. Ein Benchmarking der Bundesländer aus bildungsökonomischer Perspektive », IW-Analysen, $\mathrm{n}^{\circ}$ 10/2005

PROGNOS AG, Zukunftsatlas 2007, étude publiée en coopération avec le quotidien Handelsblatt

SACHVERSTÄNDIGENRAT ZUR BEGUTACHTUNG DER GESAMTWIRTSCHAFTLICHEN ENTWICKLUNG, Staatsverschuldung wirksam begrenzen. Expertise im Auftrag des Bundesministers für Wirtschaft und Technologie, mars 2007 (www.sachverstaendigenrat.org). 


\title{
Aufbau Ost : entre rattrapage et différenciation
}

\author{
Markus Gabel
}

La publication par l'institut Prognos de l'édition 2007 de son étude sur la compétitivité des régions allemandes (Zukunftsatlas : "Atlas du futur ») a ravivé le débat sur l'efficience et même l'opportunité de la politique de soutien aux Länder est-allemand menée depuis l'Unité. Cherchant à comprimer son déficit structurel, l'Allemagne réexamine depuis 2004 le volume comme l'orientation de la politique de reconstruction lancée après l'unification (Aufbau Ost). Si la question est complexe et prête le flanc à la polémique, elle n'est plus un sujet tabou face à un triple constat. D'un côté, cette politique a modernisé les Länder issus de l'ex-RDA : le niveau de vie de la population s'est nettement élevé, et des infrastructures d'excellente qualité ont été créées. Ces succès sont le résultat d'investissements publics très élevés, d'un soutien massif aux entreprises, d'un cadre institutionnel de bonne qualité et d'importants transferts sociaux. D'un autre côté, malgré ces efforts, le processus de rattrapage s'est arrêté depuis une décennie. Dans certains domaines, il pourrait même s'inverser à l'avenir, car les perspectives de croissance de l'est paraissent limitées, nourrissant la crainte de voir émerger un mezzogiorno allemand. Enfin, si on excepte la Saxe (et depuis 2006 le Mecklembourg), la situation financière des nouveaux Länder est désastreuse ; elle risque de s'aggraver depuis l'élargissement de l'UE et la réorientation des fonds structurels. Quel peut être dans ce contexte l'avenir des nouveaux Länder? Et comment redéfinir la politique de l'Aufbau Ost ?*

L'année 2004 a marqué un tournant dans la perception économique de la réunification. Une série de rapports pointait du doigt les sommes colossales dépensées depuis 1990, pour de bien piètres résultats. Dès 2003, Hans-Werner Sinn, président de l'institut ifo, commentait ainsi les indicateurs de l'est : "ce ne sont pas des chiffres qui génèrent l'espoir d'un rattrapage ». Mais le grand public n'a été alerté qu'en avril 2004 par le rapport très critique du groupe von Dohnanyi institué par le gouvernement Schröder afin de donner un nouveau souffle à l'Aufbau Ost. Au-delà d'une analyse sans tabou de la situation, le rapport exprime surtout ce qui semblait inconcevable jusque là : repenser la philosophie de l'Aufbau Ost, mettre fin aux subventions massives non ciblées et créer une « zone franche» (Sonderwirtschaftszone Ost).

Ce constat est plus ou moins partagé par d'autres rapports de toutes obédiences et origines : Conseil des Sages, DB-Research, Fondation Friedrich-Ebert ou Groupe de travail sur une politique économique alternative (Arbeitsgruppe 'Alternative Wirtschaftspolitik') de l'université de Brême. Par contre, le consensus s'arrête quand s'il s'agit d'esquisser une stratégie future : lutter, par une meilleure efficacité de la politique d'aides, contre le risque d'une 'mezzogiornisation' de l'Allemagne de l'est ou accepter la tendance globale et nuancer, voire abandonner l'objectif d'une harmonisation des conditions de vie comme le préconise le Conseil des

\footnotetext{
*Version actualisée d'un article paru dans Regards sur l'économie allemande, nº 71, mai 2005.
} 
Sages, ou encore encourager la population à déménager, comme le suggère, mi-ironique, misérieux, le président de l'institut DIW?

Ces divergences ne sont compréhensibles que par l'ampleur de ce qui apparaît comme un 'marasme' est-allemand lorsqu'on considère les nouveaux Länder comme un tout (« exRDA »). Ses causes remontent aux choix faits au moment de la réunification, dont la création, le 1er juillet 1990, de l'union économique, monétaire et sociale (UEMS). Seule réponse adéquate au plan administratif et politique (une intégration graduelle, par exemple la coexistence de deux monnaies, aurait été trop complexe à gérer, sans parler du coût psychologique pour la population et du flux migratoire vers l'ouest), l'UEMS a généré un coût économique considérable, car elle n'était pas adaptée à la réalité économique des nouveaux Länder.

Indicateurs économiques et sociaux de l'Allemagne de l'est - 1991-2006 (ouest = 100)

\begin{tabular}{|lrrrc|}
\hline Indicateurs économiques & $\mathbf{1 9 9 1}$ & $\mathbf{1 9 9 7}$ & $\mathbf{2 0 0 3}$ & $\mathbf{2 0 0 6}$ \\
PIB par tête (base de prix : 1995) & 49,4 & 67,1 & 66,4 & 68,9 \\
PIB par tête nominal & 33,4 & 62,2 & 63,9 & 67,9 \\
Investissements d'équipement par tête & 62,0 & 108 & 89,0 & nd. $^{\text {a) }}$ \\
Productivité (PIB par actifs) & 51,2 & 72,5 & 76,8 & 77,8 \\
Coût salarial unitaire & 141,4 & 108,7 & 100,5 & 99,6 \\
Stock de capitaux par tête & 38,0 & 66,0 & 71,0 & nd. $^{\text {a) }}$ \\
Taux d'exportation & 52,0 & 44,0 & 60,0 & nd. $^{\text {a) }}$ \\
Infrastructures publiques par tête & 50,0 & 64,0 & 78,0 & nd. $^{\text {a) }}$ \\
Indicateurs sociaux & $\mathbf{1 9 9 1}$ & $\mathbf{1 9 9 7}$ & $\mathbf{2 0 0 2}$ & $\mathbf{2 0 0 6}$ \\
Revenu net par ménage & 54 & 82 & 81 & nd. $^{\text {a) }}$ \\
Salaires & 49 & 76 & 81 & nd. ${ }^{\text {a) }}$ \\
Temps de travail (par an et par tête) & 99 & 110 & 110 & nd. $^{\text {a) }}$ \\
Taux d'activité & 96 & 91 & 87 & 90 \\
Taux de chômage & 216 & 203 & 254 & 215 \\
Budget social en \% du PIB généré & 184 & 156 & 164 & nd. ${ }^{\text {a) }}$ \\
Budget social par tête & 61 & 95 & 100 & nd. ${ }^{\text {a) }}$ \\
\hline
\end{tabular}

Sources : Arbeitsgruppe Soziale Marktwirtschaft (2004), Destatis (www.destatis.de). (a) Nd. : donnée non disponible.

Compte tenu de la faible productivité (aujourd'hui, on sait qu'elle n'atteignait que $35 \%$ de celle de l'ouest) comme des pertes d'emploi et d'actifs liées à la privatisation des ex-entreprises d'Etat, l'extension à l'est du modèle social de la RFA a présumé des forces du système social allemand qui, avec ses allocations chômage et son niveau de retraites et d'aide sociale généreux, avait dès la fin des années 1980 commencé à toucher ses limites malgré le succès du modèle allemand de l'époque (Bourgeois, 2005). Plutôt que de l'appliquer à l'est en l'état, il aurait fallu le réformer au préalable; les réformes ne se sont esquissées qu'avec l'annonce de l'Agenda 2010 par le chancelier Schröder (2003). La seconde erreur a été la mauvaise allocation du capital suite aux subventions trop massives en faveur du BTP. Elle en a d'abord alimenté artificiellement le boom, et retardé depuis 1996, début de la crise du secteur, la reprise générale. Enfin, dernière erreur : l'évolution des salaires, négociée par les partenaires sociaux, et qui, sur la base de chiffres soit inexistants, soit trop optimistes quant à la productivité, s'est soldée par un alignement beaucoup trop rapide, dommageable à l'activité et à l'emploi (Schrœeder, 2005).

Les erreurs des premières années ne doivent néanmoins pas faire oublier que l'Allemagne de l'est n'a pas connu la plupart des problèmes de la transition, tels qu'on les a observés chez 
les voisins est-européens : privatisations retardées, crise monétaire et fiscale, crise politique, etc. Outre l'UEMS, les deux facteurs-clefs de ce succès sont la rapide création administrative et institutionnelle des nouveaux Länder, ainsi que le renouveau des infrastructures. L'instrument financier en a été le Pacte de solidarité Solidarpakt I, entré en vigueur en 1995 et qui, soutenu par des fonds structurels européens, a versé jusqu'à 2004 plus de 100 milliards $€$ aux nouveaux Länder. Le net retard par rapport à l'ouest en termes de capitaux par tête s'est ainsi réduit : son niveau est passé de $38 \%$ à $66 \%$ entre 1991 et 2001.

\section{L'est entre paradoxes et dilemmes}

Tous les experts partagent aujourd'hui l'analyse selon laquelle le processus de rattrapage économique s'est arrêté depuis dix ans. Les indicateurs dressent une image cruelle. Certes, la productivité (par actif) suggère un niveau honorable : la tendance, bien que ralentie, est toujours haussière, et la productivité par actif atteint un peu plus de $77 \%$ du niveau de l'ouest en 2006. Mais l'indicateur économiquement plus parlant est celui de la productivité par actif occupé. Il reflète l'évolution sur le front de l'emploi où le nombre de salariés est passé de 8,4 millions au début de la réunification à 7,2 millions quinze ans plus tard. Ainsi, la productivité n'a jamais dépassé $61 \%$. Elle est même en baisse depuis 1999 et se situe maintenant à $59 \%$, voire $56 \%$ si on ne tient pas compte de l'emploi public (près de $15 \%$ à l'est contre à peine $5 \%$ seulement à l'ouest). En même temps, les salaires sont montés en moyenne jusqu'à environ $81 \%$ du niveau de l'ouest. Certains biens et services étant moins chers à l'est de l'Elbe, le pouvoir d'achat réel atteint environ $90 \%$ du niveau occidental.

Mais ce paradoxe (faible productivité, pouvoir d'achat élevé) n'est pas le seul : le niveau des retraites est particulièrement élevé dans les nouveaux Länder, grâce notamment à l'activité professionnelle généralisée des femmes du temps de la RDA. Si on ne tient pas compte des retraites des fonctionnaires ni des retraites d'entreprises (plus répandues à l'ouest), les sommes versées par les seules caisses d'assurance retraite légale par tête aux retraités estallemands dépassent le niveau occidental de $12 \%$ en termes nominaux et de $21 \%$ en termes réels. Si on prend également en compte les programmes de préretraites (au début de la réunification, environ un demi million de personnes a ainsi mis fin à sa vie active), on voit apparaître là un des principaux leviers de cette forte augmentation des cotisations sociales outre-Rhin depuis 1995 (plus de 5 points de pourcentage par rapport au PIB) qui sapait le pouvoir d'achat des Allemands ainsi que la capacité de relance de l'économie allemande.

A l'est, le problème fondamental - depuis la réunification - est que la production propre des nouveaux Länder est toujours restée en dessous de leur consommation de biens et services. Malheureusement, la tendance s'accroît. Entre 1991 et 2002, le déficit de la balance des paiements des cinq Länder orientaux est passé de 80 milliards $€$ à 113 milliards $€$ : en 2002, pouvoirs publics, investisseurs privés et ménages ont acheté des biens et services d'une valeur de 366 milliards $€$, mais leur production n'a atteint qu'un volume de 253 milliards $€$. La différence est financée pour trois quarts par des transferts publics et pour un quart par des investissements directs et de l'endettement.

Conséquence de subventions peu ciblées, d'importantes surcapacités ont été accumulées, notamment dans le secteur du logement. A cela s'ajoute aujourd'hui que non seulement les capacités de production occidentales sont souvent suffisantes pour satisfaire la demande orientale, mais aussi que depuis la fin du boom des premières années de l'unité, les entreprises 
ouest-allemandes préfèrent investir à l'étranger où les marges de bénéfices sont nettement plus élevées qu'à l'est. Même si le désavantage en termes de coûts salariaux s'est réduit ces dernières années, les rendements restent handicapés à l'est par une série de facteurs : production désavantagée par des petits volumes de production et des dépenses d'énergie élevées, niveau pénalisant des charges fiscales et coûts d'approvisionnements, surtout par rapport aux voisins d'Europe de l'est. La région est littéralement prise en sandwich entre l'Allemagne de l'ouest, très productive et taillée pour être leader sur de nombreux marchés internationaux, et les nouveaux Etats membres de l'Union européenne, moins chers et plus dynamiques. L'investissement a ainsi peu d'intérêt à affluer entre l'Elbe et l'Oder, surtout quand il s'agit de créer des entreprises dans les secteurs intensifs en main d'œuvre (en atteste le recul des investissements d'équipements qui n'atteignent plus que $89 \%$ du niveau occidental, contre $108 \%$ en 1997).

Ce manque de perspectives pour l'investissement, combiné à une évolution démographique très négative - l'émigration vers l'ouest et la forte baisse des naissances rendent les conséquences du vieillissement encore plus dramatiques que pour le reste de l'Allemagne font craindre à nombre d'observateurs que l'est ne soit pris dans une spirale descendante comme on l'observe depuis des décennies pour l'Italie du Sud.

Cette comparaison peut surprendre, car historiquement, les deux régions ont peu en commun. Avant la guerre, l'Allemagne de l'est représentait un noyau central de l'industrialisation allemande. La région avait profité d'une nette ascension pendant l'économie de guerre des national-socialistes et avait été moins touchée par les bombardements que les régions occidentales. Entre 1936 et 1944, la production y avait augmenté de plus de $50 \%$, permettant d'assurer près de $30 \%$ des biens de base allemands. Et, surtout, la productivité y était supérieure de $27 \%$ à celle de l'ouest. Le sud de l'Italie n'a jamais connu de telles évolutions. Pourtant, les deux régions présentent aujourd'hui un profil similaire : en termes de PIB par tête, le sud de l'Italie atteint à peine $60 \%$ du reste du pays (66\% dans le cas des nouveaux Länder). La productivité ( $83 \%$ contre $75 \%$ ) est relativement plus favorable du côté italien. Même chose pour la structure salariale : en Allemagne de l'est, l'emploi industriel ne représente que $15 \%$ du total contre $19 \%$ en Italie du sud.

Ces chiffres ne doivent néanmoins pas faire oublier une autre réalité du sud de l'Italie : l'illettrisme, une grande pauvreté et des structures maffieuses qui pénalisent son développement. Ces handicaps particuliers se traduisent par un très faible taux d'exportation : il n'atteint que $11 \%$ du niveau du reste de l'Italie (le chiffre correspondant pour les nouveaux Länder est $60 \%$ ), entretenant le sous-développement de la région - une tendance que le gouvernement italien, malgré des subventions massives, surtout sur le marché du travail, n'arrive pas à inverser. Mais plus importante encore est l'absence de pôles de compétitivité dans le mezzogiorno italien. Or ces zones existent dans l'est de l'Allemagne - et non pas seulement parce que la région abrite aussi la capitale Berlin. Il faut rappeler dans ce contexte que la présence de régions à faible productivité est le propre de tous les grands pays industrialisés ; leur absence outre-Rhin était justement ce qui avait longtemps différencié l'Allemagne de ses voisins.

Il n'en reste pas moins que, plus de 15 ans après l'unification, le PIB par tête n'atteint toujours à l'est que les deux tiers du niveau occidental. Mais cette moyenne masque une forte homogénéité. Certes, la différence entre les PIB des cinq Länder est minime, mais c'est là l'effet de lissage de l'aide financière de l'ouest, notamment dans le cadre du système de 
péréquation entre les Länder (Länderfinanzausgleich). Une raison fondamentale de l'absence d'une dynamique autonome à l'est est la faiblesse du tissu de PME. Seule la Saxe, aidée par sa tradition et une politique qui a misé très tôt sur ce vecteur, a réussi le maillage entre grands groupes et PME si typique du capitalisme rhénan. La Thuringe n'en est pas loin grâce au pôle d'excellence autour de Jenoptik (Guiot, 2006). Mais ailleurs, les PME manquent. Ainsi, en Saxe-Anhalt, l'héritage de la pétrochimie à Leuna et de l'industrie chimique à Bitterfeld a très longtemps monopolisé toutes les énergies. Il commence seulement à générer des pôles de croissance. Faute de tradition industrielle, la situation est plus contrastée dans le Mecklembourg, ainsi que dans le Brandebourg, après l'échec de plusieurs grands projets.

\section{Coût élevé de l'Aufbau Ost et finances publiques en déficit}

En avril 2004, une couverture de l'hebdomadaire Der Spiegel faisait sursauter l'Allemagne : " 1250 milliards pour quoi faire? ". Peu après, le ministre fédéral des Transports tentait de calmer les esprits en chiffrant le véritable coût de l'Aufbau Ost à 250 milliards $€$. D'où vient une telle divergence ? Le chiffre de 1250 milliards $€$ est basé sur les estimations de l'institut de Halle (IWH) pour la période de 1991 à 2003. En incluant 1990 et 2004, on obtient 1500 milliards $€$. Il s'agit du total des transferts bruts, donc des sommes versées à l'est, financées par le Bund, les Länder de l'ouest et le système de protection sociale. Outre les moyens affectés aux besoins spécifiques de l'est (par exemple la reconstruction des infrastructures), ce total inclut également des dépenses classiques (comme les salaires des fonctionnaires des nouveaux Länder) et les transferts sociaux comme les allocations chômage ou les retraites.

Ce calcul ne prend pas en compte que les nouveaux Länder contribuent eux aussi à ces transferts - via les impôts et les cotisations sociales ; mais leur faiblesse économique limite leur contribution à leurs propres besoins. Il serait ainsi plus judicieux de considérer les transferts nets. Les nouveaux Länder contribuent à hauteur de 8 à $10 \%$ aux recettes fiscales allemandes (10\% pour la TVA, $4 \%$ pour l'impôt sur le revenu et $1 \%$ pour l'impôt sur les sociétés). Selon l'IWH, les transferts nets s'élèvent ainsi à 83 milliards $€$ en 2003 , dont $45 \%$ pour le domaine social, $13 \%$ pour les infrastructures, $9 \%$ pour l'investissement, le reste pour des réalisations diverses. Pour l'ensemble de la période 1990-2004, les transferts nets se situent alors à près de 1200 milliards $€$, financés notamment par la contribution de solidarité (Solidaritätszuschlag) qui majore l'impôt sur le revenu de 5,5 points de pourcentage, l'augmentation des cotisations sociales et le gel, voire la baisse des dépenses dans les collectivités ouest-allemandes. C'est cette somme qu'il faut prendre en considération si on se borne à une perspective de pouvoir d'achat.

Les montants alloués à la reconstruction de l'est au sens strict, qu'ils soient destinés aux travaux d'infrastructure ou aux entreprises, sont, quant à eux, nettement plus faibles. Jusqu'à la fin 2004, leur total s'élève à un peu moins de 270 milliards $€$, c'est-à-dire environ $18 \%$ des transferts bruts (pour comparaison: le budget français de 2004 s'élève à 283 milliards $€$ ). Cette relation relativise le débat quant à l'efficacité des aides, car la large majorité des sommes qui sont allées à l'est a toujours été destinée à la consommation et non à l'investissement. Dans le même temps, ce constat a de quoi inquiéter, puisqu'il révèle aussi la faiblesse inhérente à la politique de transferts, que les instituts chargés de l'évaluation de l'Aufbau Ost critiquent en rappelant que les « effets addictifs » des subventions ont incité et incitent toujours les nouveaux Länder à vivre au-dessus de leurs moyens. 


\section{Solidarpakt I et Solidarpakt II}

En 1995, le fédéralisme financier avait été élargi d‘un Pacte de solidarité afin d'encadrer l'effort budgétaire supplémentaire nécessaire pour intégrer les nouveaux Länder dans le système interrégional de péréquation. Il avait été conçu pour une durée de 10 ans, et les Länder de l'est ont perçu 105 milliards $€$ dans le cadre du Solidarpakt I (1995-2004).

Au début 2005, le Solidarpakt II a pris le relais. Etabli pour une durée de 15 ans, il porte sur un volume de 156 milliards $€$. Contrairement au premier pacte, l'aide est maintenant dégressive et oblige les Länder à consolider leur situation financière. L'aide est divisée en deux pôles : le pôle 1 prévoit 105 milliards $€$ dans le cadre des Sonderbedarfsbundesergänzungszuweisungen (SoBEZ), des subventions complémentaires du Bund destinées à rattraper le retard dans les infrastructures et à soutenir les budgets des communes à capacité financière inférieure à la moyenne (unterproportionale Finanzkraft). D'un niveau de 10,5 milliards $€$ en 2005, son niveau baisse annuellement (surtout à partir de 2009) pour s'établir à 2,1 milliards $€$ en 2019, dernière année de versement. En contrepartie de cette dégressivité, les Länder de l'est ont obtenu que le gouvernement fédéral renonce de fait à son droit de codécision quant à l'utilisation des fonds - avec comme conséquence que les Länder utilisent l'argent pour des fins autres que le but d'origine. Seule la Saxe réalise une utilisation conforme au Solidarpakt II. Le pôle II porte sur un volume de 51 milliards $€$. Il contient également les moyens provenant des Fonds structurels européens. Le niveau est négocié annuellement selon la situation financière du Bund. Pour limiter l'incertitude, les Länder de l'est souhaitent que cette deuxième partie du Solidarpakt II soit inscrite dans la Loi fondamentale. Cette question est à l'étude dans le cadre des discussions sur la réforme du fédéralisme financier, engagées depuis le début 2007.

\begin{tabular}{|c|c|c|c|c|c|}
\hline & Saxe-Anhalt & Mecklembourg-P. & Saxe & Brandebourg & Thuringe \\
\hline \multicolumn{6}{|c|}{ Utilisation des $\operatorname{SoB} E Z$ pour investissements (en \%) } \\
\hline 2002 & $-9,2$ & 6,5 & 68,7 & 15,6 & 19,8 \\
\hline \multirow[t]{2}{*}{2003} & $-13,8$ & 8,3 & 86,3 & 16,7 & 16,2 \\
\hline & \multicolumn{5}{|c|}{ Utilisation des $S o B E Z$ pour investissements et capacités financières inférieures (en \% } \\
\hline 2002 & 7,9 & 23,9 & 86,3 & 34,8 & 37,4 \\
\hline 2003 & 3,7 & 25,8 & 103,8 & 34,2 & 33,7 \\
\hline
\end{tabular}

Malgré un montant finalement assez modeste - les transferts nets de 83 milliards $€$ en 2003 représentent à peine $4 \%$ du PIB -, le volume des aides aux nouveaux Länder est de plus en plus critiqué. Les adversaires avancent que ces transferts « épuisent » à long terme le PIB car leur niveau dépasse celui de la croissance annuelle. Cela est certes vrai. Mais la réalité est plus complexe, car ces transferts impliquent une importante redistribution en faveur de certains compartiments du secteur privé : ils sont complétés par un autre flux, celui des biens et services fournis essentiellement par les entreprises ouest-allemandes.

L'est prend ainsi une place importante dans le circuit de production des entreprises ouestallemandes. En 2000, le DIW avait estimé que les transferts exercent durablement sur le PIB ouest-allemand un effet positif de 6 à $7 \%$ par an. L'institut IW de Cologne rejoint cette analyse. Il estime que le PIB ouest-allemand a ainsi bénéficié entre 1990 et 2000 d'une croissance supplémentaire de 145 milliards $€$ (en termes réels). Si on prend également en compte les recettes fiscales supplémentaires générées par cet immense programme conjoncturel, ainsi que les économies réalisées par les caisses sociales du fait d'un chômage moins élevé à l'ouest (si on additionne les estimations du DIW et du IW, on arrive à plus de 90 milliards $€$ : soit plus que les 83 milliards $€$ de transferts nets), il convient bien d'admettre que les transferts ouest-est sont au moins partiellement compensés par des gains à l'ouest.

Cette compensation ne réduit en rien le caractère dramatique de la situation est-allemande. En termes absolus, la dette cumulée de l'ensemble des collectivités des nouveaux 
Länder a atteint 85 milliards $€$ en 2003 - soit à peu près la somme perçue la même année au titre des transferts nets. L'endettement par tête (en intégrant la dette des communes) dans ces Länder est monté à $6274 €$ à la fin 2003. Si on y ajoute l'endettement du Bund (9 $345 €)$, l'endettement total de chaque Allemand de l'est s'élève à plus de $15600 €$. Si ce niveau reste en dessous du niveau occidental (plus de $16000 €$ en 2003), il doit être supporté par un PIB par tête qui n'atteint qu'à peine deux tiers du niveau de l'ouest.

Aspects financiers et économiques des nouveaux Länder en 2003

\begin{tabular}{|c|c|c|c|c|}
\hline & Déficit $^{\text {a }}$ & $\begin{array}{l}\text { Dette totale } \\
\text { par tête }\end{array}$ & $\begin{array}{l}\text { Soutien par des fonds } \\
\text { structurels }\end{array}$ & $\begin{array}{l}\text { Répartition des moyens } \\
\text { du Solidarpakt II }\end{array}$ \\
\hline Mecklembourg-P. & 3,32 & 6675 & 2,44 & 10,5 \\
\hline Berlin & 5,78 & - & ${ }^{\mathrm{d}} 0,73$ & 19,0 \\
\hline Brandebourg & 2,22 & 7010 & 2,98 & 14,3 \\
\hline Saxe-Anhalt & 3,1 & 7902 & 3,23 & 15,7 \\
\hline Thuringe & 2,92 & 6726 & 2,78 & 14,3 \\
\hline Saxe & 0,71 & 3917 & 4,69 & 26,1 \\
\hline Ensemble RFA & 3,7 & $n d$ & - & - \\
\hline & \multicolumn{2}{|c|}{ Croissance $^{f}$} & PIB par tête ${ }^{g}$ & Population $^{\mathrm{h}}$ \\
\hline Mecklembourg-P. & \multicolumn{2}{|c|}{0,8} & 17,1 & 1,74 \\
\hline Berlin & \multicolumn{2}{|c|}{0,4} & 20,9 & ${ }^{\mathrm{d}} 1,25$ \\
\hline Brandebourg & \multicolumn{2}{|c|}{0,9} & 17,5 & 2,58 \\
\hline Saxe-Anhalt & \multicolumn{2}{|c|}{0,9} & 17,4 & 2,55 \\
\hline Thuringe & \multicolumn{2}{|c|}{1,7} & 17,6 & 2,39 \\
\hline Saxe & \multicolumn{2}{|c|}{2,3} & 17,8 & 4,35 \\
\hline Ensemble RFA & \multicolumn{2}{|c|}{1,7} & 27,4 & 81,3 \\
\hline
\end{tabular}

Sources : Gouvernement fédéral, ministère des Finances, Destatis, Commission européenne. (a) en \% du PIB du Land ; (b) Land et communes, en millions $€$; (c) en milliards $€$; (d) Berlin-Est ; (e) en \%; (f) en 2004, en \% par rapport à 2003 (g) en milliers ; (h) en millions.

Les causes de cette situation se trouvent des deux côtés du budget. La capacité à autofinancer les dépenses est restée très basse à l'est: en 2003, la part des recettes fiscales propres permettant de couvrir les dépenses n'atteint que $34 \%$. Mais l'est dépense également trop. Si on prend les quatre Länder les plus pauvres (à l'est : le Brandebourg, le Mecklembourg, la Saxe-Anhalt et la Thuringe; à l'ouest : la Basse-saxe, la Rhénanie-Palatinat, la Sarre et le Schleswig-Holstein), on constate qu'à l'est les moyens financiers sont plus élevés de $20 \%$, tandis que le déficit par tête (toutes collectivités confondues) est plus important à l'est qu'à l'ouest (574 € contre $514 €$ en 2003). Seule exception : la Saxe, avec $142 €$ de déficit par tête. La situation des finances publiques est indéniablement très mauvaise à l'est, mais elle l'est également dans beaucoup de Länder de l'ouest.

$\mathrm{Au}$ vu de ces chiffres, il n'est guère étonnant que les nouveaux Länder utilisent de plus en plus souvent les aides du Solidarpakt I pour combler des trous budgétaires - pour assurer tout simplement les dépenses courantes comme les salaires des fonctionnaires. A plusieurs reprises, le ministre fédéral des Finances avait exprimé son fort mécontentement quant à cette utilisation peu conforme des aides à l'investissement ; mais les termes du pacte le condamnent à l'impuissance. Les tensions s'intensifient avec le Solidarpakt II qui prévoit le versement de 156 milliards $€$ aux cinq Länder de l'est sur la période 2005-2019. Ces flux n'ont en effet pas d'affectation fixe dans ce pacte non plus. 
Autre pomme de discorde : la remise en question de certaines aides de l'UE. Jusqu'ici, les nouveaux Länder percevaient environ 20 milliards $€$ par an au titre des fonds structurels européens (FSE). A partir de 2007, ce montant a certes été revu à la baisse du fait de l'élargissement de l'UE, mais cette réduction reste limitée, les critères de soutien pour la période 2007-2013 étant établis sur la base des années 2001 à 2003. Et un rapport de cohésion de la Commission européenne montrait qu'en 2001, l'ensemble du territoire est-allemand à l'exception de Berlin, Dresde, Leipzig et de certaines parties du Brandebourg, se situait audessous du seuil de $75 \%$ de la moyenne du PIB de l'UE justifiant les subventions les plus élevées (Objectif 1). Il est vrai que la phase de renégociation des FSE incitait les Länder estallemands à dramatiser quelque peu la réalité de leur retard pour prolonger les subventions... L'accord de décembre 2005 conclu entre les Etats membres sur le budget européen 20072013 a prolongé le phasing out pour les régions est-allemandes les plus en retard (Gabel, 2006); à l'inverse, celles de Halle, Leipzig et du sud-ouest du Brandebourg ne bénéficieront des aides que jusqu'en 2008 au lieu de 2009.

\section{Pourquoi l'Aufbau Ost a-t-il échoué et comment faire mieux?}

En Allemagne, on noircit volontiers le tableau quant à l'Aufbau Ost. Pourtant, il existe des secteurs et des pôles au dynamisme indéniable. Dans certains villes et régions (Leipzig, Chemnitz, Rostock, Potsdam, pour n'en citer que quelques-unes), la situation est non seulement très satisfaisante, elle est également meilleure que dans certains régions de la Sarre, de la Basse-Saxe ou même de Bavière. Néanmoins, il faut admettre que dans sa globalité, la réalité est-allemande est encore très loin de ces " paysages fleuris » promis par Helmut Kohl en 1989. La liste des explications à cet échec relatif est longue et connue : taux de conversion trop généreux, privatisations hasardeuses, salaires disproportionnés, non-affectation des transferts, etc. De même que celle des 'coupables' présumés : responsables politiques, entreprises ouest-allemandes trop peu 'patriotiques'...

Mais aurait-on pu faire mieux? Difficilement, étant donné l'urgence et l'ampleur du chantier. Et par-delà les griefs de circonstance, on peut distinguer trois raisons principales permettant d'expliquer la situation actuelle : 1) l'héritage historique et la nature même de la transition ; 2) l'évolution salariale ; 3) la dérive des pratiques de cohésion territoriale liées à l'ordre constitutionnel de la RFA.

Le débat sur le niveau et l'évaluation du soutien financier à l'est a tendance à oublier un peu hâtivement certaines données historiques ainsi que les caractéristiques inévitables du processus de transition. Après 1945, les régions est-allemandes ont subi leur première désindustrialisation massive sous le régime soviétique, se manifestant par le démontage de l'industrie et des infrastructures, évalué à un tiers de la capacité industrielle de 1944. S'y ajoutent les réparations financières, estimées à environ 13 milliards \$ (aux prix de l'époque). Le niveau des réparations par tête était ainsi quatre fois plus élevé pour les citoyens de l'exRDA que pour ceux de la RFA. Après 1990, l'est a subi une deuxième désindustrialisation suite à la restructuration et à la dissolution des anciennes entreprises d'Etat dans le cadre des opérations de la Treuhandanstalt. D'autres facteurs s'y sont ajoutés, dont la perte des marchés en Europe de 1'Est. Au total, la production industrielle est-allemande a ainsi baissé de près de deux tiers entre 1989 et 1992 . Même en 1997, elle n'atteignait qu'à peine $50 \%$ du niveau d'avant l'Unité. Malgré les spécificités est-allemandes, cette évolution est typique et 
probablement inévitable : en effet, le PIB de presque tous les pays en transition a traversé une courbe en ' $U$ ' avec un fort effondrement après la libéralisation économique, suivi d'un rattrapage progressif.

Le second facteur expliquant l'échec de la réunification économique est le niveau des salaires et le rattrapage trop rapide. Souvent, cette tendance est justifiée par le taux de conversion très favorable de l'Ostmark en DM. Cependant, malgré un taux de près de la parité, les salaires orientaux n'atteignaient qu'environ un tiers du niveau occidental au début des années 1990. Le gros du rattrapage s'est joué après. C'est d'autant plus regrettable, car avec des salaires à un tiers du niveau de l'ouest, l'est aurait facilement concurrencé toute l'UE de l'époque et attiré massivement des investissements étrangers - avec pour conséquence que les salaires auraient remonté tout naturellement, mais avec une productivité qui aurait eu le temps de suivre.

La réalité a malheureusement été autre, car presque tout le mouvement de rattrapage s'est réalisé dans la première moitié des années 1990. Il a ainsi devancé sensiblement l'évolution de la productivité. La responsabilité de cette évolution est partagée entre les acteurs politiques et les partenaires sociaux : le patronat ouest-allemand pour avoir cherché à contenir la « concurrence déloyale » des entreprises est-allemandes, les syndicats pour avoir cherché à enrayer le risque d'un «dumping social», et le pouvoir politique pour avoir préparé le terrain en se mêlant massivement aux les négociations conformément au slogan « à travail égal, salaire égal ».

Plus encore que les deux premiers facteurs, c'est la dérive (antérieure à l'Unité) des pratiques liées à l'ordre constitutionnel de la RFA qui explique l'échec relatif de la politique de l'Aufbau Ost. Celui-ci repose sur les principes organisant la cohésion territoriale. Le premier est celui des « tâches communes » (Gemeinschaftsaufgaben) qui permet de déroger dans certains cas à la répartition des compétences législatives entre le Bund et les Länder au nom de l'intérêt conjoint. L'art. 91 a $\S 1$ de la Loi fondamentale stipule ainsi que le Bund «concourt à l'accomplissement des tâches des Länder dans les domaines suivants, si ces tâches sont importantes pour l'ensemble et si ce concours ... est nécessaire à l'amélioration des conditions de vie ». Ce concours est d'ordre financier : le Bund contribue à hauteur de $50 \%$ aux dépenses nécessaires.

Les domaines concernés sont au nombre de trois, dont "l'amélioration de la structure économique régionale » (Verbesserung der regionalen Wirtschaftsstruktur). Voilà qui légitime les aides à l'investissement pour les entreprises et les collectivités. L'autre principe-clef est celui de la solidarité territoriale à la base du système de péréquation financière dans le cadre du fédéralisme financier (Länderfinanzausgleich), appliqué dans le Solidarpakt I et II. Or il apparaît aujourd'hui que ni l'un ni l'autre de ces principes ne se sont révélés adaptés à la reconstruction de la structure économique est-allemande, car leur mise en œuvre avait subi au fil des ans une dérive favorisant une pratique de saupoudrage indifférencié des aides plutôt qu'une politique de soutien ciblé. Et ils se sont traduits par une série d'effets pervers qui inhibent aujourd'hui la dynamique de croissance des nouveaux Länder (et de certains Länder ouest-allemands) au lieu de la libérer.

Les Gemeinschaftsaufgaben, inscrites dans la Loi fondamentale à la fin des années 1960, ont toujours été une politique qui visait des territoires très limités, ainsi que l'harmonisation des conditions de vie entre des territoires où les disparités étaient somme toute assez faibles. Lors de la réunification, on a appliqué cette politique à une région avec des problèmes d'une 
tout autre nature. L'ex-RDA a des structures qui sont beaucoup moins favorables au succès de cette politique car la région dispose de peu de zones à forte concentration. Malgré ce fait, l'Aufbau Ost se base jusqu'à ce jour sur l'attribution uniforme, sans considération des différences régionales, de volumes financiers redistribués dans le cadre du fédéralisme financier. Outre le fait que les Gemeinschaftsaufgaben contreviennent à l'organisation du fédéralisme qui, dans le partage des compétences, confie l'autonomie administrative aux Länder (leur part a d'ailleurs été réduite dans le cadre de la réforme du fédéralisme politique de 2005), ces « tâches communes », de même que le Länderfinanzausgleich, ont non seulement généré une attitude revendicatrice de la part des Länder qui en bénéficient, mais les incitent également, en socialisant le financement de certaines tâches, à un comportement d'autant plus dépensier qu'aucun Pacte de stabilité interne à la RFA ne les contraint à respecter une certaine discipline budgétaire. Le souhait des nouveaux Länder de faire inscrire le Solidarpakt II dans la Loi Fondamentale en est une illustration.

La politique de subventionnement massif est également le fruit de la dérive d'un autre principe constitutionnel : la "réalisation de conditions de vie équivalentes 》 (art. 72 §) qui légitime l'intervention législative du Bund au nom de l'intérêt supérieur de "l'ensemble de l'Etat " dans le cadre des compétences législatives concurrentes qui confèrent aux Länder "le pouvoir de légiférer aussi longtemps et pour autant que la Fédération n'a pas fait par une loi usage de sa compétence législative » (art. 72 § 1). Cet article, qui avait contribué à une centralisation rampante des compétences, s'appliquait nécessairement lors de l'unification au nom de la solidarité nationale.

Ce dernier principe confère en outre au Bund le droit de rééquilibrer les capacités financières entre les Länder en leur accordant des dotations complémentaires afin de «les aider à couvrir leurs besoins financiers généraux (dotations complémentaires) 》(art. $107 \S 2$ al. 3) - mais sans qu'il soit question de conditions de vie « équivalentes ». Or c'est l'interprétation à accorder à cette dernière notion qui fait débat. A l'origine, le qualificatif figurant dans la Constitution était : « homogènes » (Einheitlichkeit). Or au fil du temps, l'interprétation de ce concept avait abouti à une dérive égalitariste vers l'idée d'uniformité des conditions de vie. L'article 107 de la Loi fondamentale avait dès lors été modifié en 1994, et le qualificatif « homogènes » remplacé par « équivalentes » afin de rendre plus transparent l'esprit de ce principe, à savoir l'équité des chances. Et dans plusieurs arrêts rendus depuis, le Tribunal constitutionnel fédéral a rappelé qu'il implique l'acceptation de certaines disparités et ne peut donc se confondre avec une approche égalitaire, considérée comme contraire aux principes fondateurs de l'ordre constitutionnel libéral et démocratique de la RFA. Pour mieux expliciter ce retour à l'orthodoxie, la Cour avait par exemple formulé le principe de "l'interdiction du nivellement " (Verbot der Nivellierung) dans son arrêt du 11-11-1999 sur le Länderfinanzausgleich.

$\mathrm{Au}$ niveau fédéral, la remise en cause de cette politique de soutien indifférencié est récente. Avec une croissance qui, depuis 1996, reste globalement au-dessous du niveau de l'ouest (entre 1996 et 2001, le PIB a progressé de 1,5\% par an contre 1,7 \% à l'ouest), la région n'aura pas les moyens de se hisser au même niveau que l'ouest. Car à long terme, ce n'est pas la demande (largement subventionnée), mais l'offre qui suscitera la croissance. Cette offre est déterminée par le travail et le capital disponibles, ainsi que le progrès technique. Même si on suppose que le stock de capital s'accroît à l'est (il ralentit depuis 1995), l'évolution démographique condamne la région : sa population diminue et vieillit plus rapi- 
dement que celle de l'ouest. Le rattrapage qui ne s'est pas réalisé jusqu'ici pourra difficilement se faire à l'avenir. Mais ceci ne condamne pas la région dans son entier : comme à l'ouest, la différenciation territoriale s'accroîtra à l'est ; seules quelques zones atteindront le niveau de l'ouest.

L'Allemagne cherche à donner un nouveau souffle à l'Aufbau Ost. Si la proposition de constituer une Sonderwirtschaftszone Ost (une sorte de zone franche) revient régulièrement dans le débat (mais sa réalisation est peu compatible avec la réglementation européenne), la tendance est plutôt à la concentration des aides dans des clusters, c'est-à-dire des zones à grappes d'activités spécialisées et ouvertes à la concurrence internationale. La Saxe et la Thuringe ont montré la voie. Les chiffres sont parlants : en 2004, la Saxe enregistrait la plus forte croissance $(2,3 \%)$ des Länder allemands. En 2006, avec $4 \%$, elle s'est hissée au rang de championne d'Allemagne. Il n'est guère étonnant dès lors que la large majorité des rapports d'experts préconise la concentration des aides dans des clusters, d'autant que cette approche a fait par ailleurs ses preuves en Bavière ou en Rhénanie du Nord-Westphalie.

Après la Saxe et la Thuringe, le Brandebourg et le Mecklembourg se sont mis eux aussi à exploiter leurs zones à fort potentiel de croissance en se positionnant dans l'énergie solaire et éolienne, ainsi que dans l'agroalimentaire. Cette stratégie est portée par le retour de la croissance outre-Rhin et notamment l'excellence des résultats à l'export des entreprises allemandes. Le secteur industriel à l'est, qui commence enfin à bénéficier d'un coût salarial unitaire inférieur à celui à l'Ouest, est le principal bénéficiaire de cette tendance. Ainsi, en 2006, pour la première fois depuis longtemps, le nombre d'actifs à l'est s'est inscrit à la hausse, et la croissance de l'est (3\%) a dépassé celle à l'ouest (2,6\%). Cette croissance reste néanmoins insuffisante pour accélérer le processus de rattrapage de l'est, car la baisse de la population n'a jamais cessé depuis 1990 et demeure sur une tendance stable avec un recul se situant autour de $-0,7 \%$ par an.

LA DÉCEPTION CONCERNANT L'AUFBAU OST est nourrie par deux facteurs : d'un côté l'optimisme démesuré quant à la rapidité du rattrapage qui avait prévalu au cours des premières années de l'unité allemande ; de l'autre, une confiance trop aveugle dans le système politique, économique et en particulier social, qui a voulu ignorer trop longtemps la nécessité de réformes structurelles.

De ce point de vue, et bien que les réformes aient été engagées, l'échec de l'Aufbau Ost reste le point de cristallisation des deux principaux maux allemands : un système social trop généreux compte tenu d'une capacité économique longtemps bridée et d'une société vieillissante, d'une part, et un système fédéral dont l'approche liant concurrence et solidarité à la fois s'est dénaturée au fil du temps, aboutissant à une centralisation rampante et à un système de péréquation financière qui incite trop à l'assistanat et pas suffisamment au développement de la compétitivité économique. Ces problèmes sont identifiés, et de timides réformes sont engagées.

Quant à l'est - et finalement à l'Allemagne tout entière - un autre phénomène s'y ajoute. Le «modèle allemand» ne pourra plus à l'avenir garantir le même niveau 'd'égalité' que celui auquel les Allemands se sont habitués pendant si longtemps. Face à ses difficultés budgétaires et mue par son cap de réformes dans le cadre de la Stratégie de Lisbonne, l'Allemagne rompt avec la dérive égalitaire, source de nivellement. Finalement, c'est un processus de normalisation qui traverse aujourd'hui l'Allemagne - une normalisation structurelle 
impliquant l'acceptation des disparités qui existent également à l'ouest et dont la croyance en ces fameux "paysages fleuris » avait masqué un peu la perception.

A l'est, seules certaines zones ont déjà réussi leur rattrapage ou y parviendront, d'autres resteront durablement à la traîne. Cette évolution est inévitable compte tenu des mutations démographiques. Le scénario développé pour l'est en 2020 par le Berlin-Institut paraît dans ce contexte très réaliste : «En 2020, on pourra se promener entre la mer Baltique et le Fichtelgebirge sans rencontrer beaucoup de personnes... Avec le vieillissement, la nature reprendra ses droits. Elle enclenchera une dynamique écologique sans précédant dans l'histoire moderne de l'Allemagne. La diminution de la population et sa concentration dans des régions à forte plus-value modifiera la conception de l'espace. D'importantes sommes financières deviendront disponibles et pourront être réorientées vers des utilisations plus productives, comme l'enseignement et la recherche». Mais ce scénario s'appliquera aussi à de larges territoires du Schleswig-Holstein, de la Basse-Saxe ou même de la Bavière...

\section{Indications bibliographiques}

AG 'Alternative WiRtschaftSPOLITIK', « Gegen die Zwangsperspektive des ostdeutschen Zurückbleibens - für forcierte Mobilisierung endogener Entwicklungspotentiale Ost », Universität Bremen, 2004

BACH S., VESPER D., «Finanzpolitik und Wiedervereinigung. Bilanz nach 10 Jahren », DIW-Vierteljahresheft, $\mathrm{n}^{\circ} 2 / 2000$

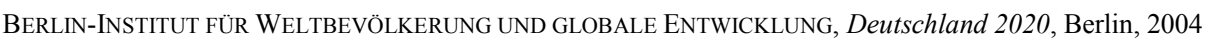

BOURGEOIS I., « 10 ans après l'unification: l'économie des nouveaux Länder en voie de normalisation », Regards sur l'économie allemande, $\mathrm{n}^{\circ} 46 / 00$, et «Quelle politique pour les nouveaux Länder dans l'UE 25 ?», Regards sur l'économie allemande, $\mathrm{n}^{\circ} 61 / 03$

Bourgeois I., « La difficile gestation des réformes », in BOURGEOIS I. (dir.), Le modèle social allemand en mutation, Cirac, Cergy-Pontoise, 2005

BUNDESREGIERUNG, Jahresbericht 2004 zum Stand der deutschen Einheit, 2004

Busch U., Am Tropf. Die Ostdeutsche Transfergesellschaft, Berlin 2002

DB RESEARCH, « Perspektiven Ostdeutschlands, 15 Jahre danach », n 306, 2004

DIW / IAB / IfW / IWH / ZEW, Fortschritte beim Aufbau Ost. Fortschrittsbericht wirtschaftswissenschafticher Institute über die wirtschaftliche Entwicklung in Ostdeutschland, 2002

DRÜKE H., «Europas Stiefel drückt und zwickt - Grundprobleme der Wirtschaft Italiens », Aus Politik und Zeitgeschichte, B 35-36, p. 23, 2004

FES-MANAGERKREIS, Doch noch ein Wirtschaftswunder in Ostdeutschland ?, mai 2004

GABEL M., « La réforme du fédéralisme financier allemand: beaucoup de bruit pour rien? », Regards sur l'économie allemande, $\mathrm{n}^{\circ} 52-53$, octobre 2001

GABEL M., «Budget européen : l'Allemagne se repositionne », Regards sur l'économie allemande, $\mathrm{n}^{\circ} 75$, mars 2006

Guiot C., «Carl Zeiss AG, ou : comment l'innovation a résisté au Mur », Regards sur l'économie allemande, n 79, décembre 2006

LALLEMENT R., «L'économie est-allemande : transition inachevée et réorientation de la politique économique », Note du Cerfa, $\mathrm{n}^{\circ} 17$, novembre 2004

LAMMERS K., " Problemregion Ostdeutschland - was ist zu tun? », IW-Wirtschaftsdienst, n 10/2004

RAGNITZ J., « Zur Ausgestaltung des Solidarpaktes II »“, IWH Diskussionspapier, n 194/2004

RAGNITZ J., « Transferleistungen für die neuen Länder », Wirtschaft im Wandel, n 9-10/2004

SACHVERSTÄNDIGENRAT, Jahresgutachten 2002-2003 et 2004-2005

SCHROEDER W., « Le modèle syndical allemand n'existe plus' », in BOURGEOIS I. (dir.), Le modèle social allemand en mutation, Cirac, Cergy-Pontoise, 2005

SINN H.-W., Ist Deutschland noch zu retten?, Econ, 2003. 
- II -

PORTRAITS

DE LÄNDER 



\title{
Rhénanie du Nord-Westphalie : reconversion du charbon au high tech
}

\author{
Isabelle Bourgeois
}

La Rhénanie du Nord-Westphalie - première économie régionale d'Allemagne - est toujours le premier site producteur d'acier outre-Rhin. Comment pourrait-il en être autrement de cette région qui fut le berceau de l'industrialisation allemande ? Mais c'est aussi un des sites les plus tertiarisés et qui, de surcroît, talonne la Bavière en matière d'innovation. Elle a connu comme cette dernière une longue et profonde transformation. Mais si la Bavière, en un petit demisiècle, est passée de l'agriculture à l'industrie, puis au high-tech sans négliger son artisanat, la Rhénanie du Nord-Westphalie a suivi un cheminement quelque peu différent, misant sur ses infrastructures, mais aussi sur l'image porteuse que lui confère une concentration unique de médias. *

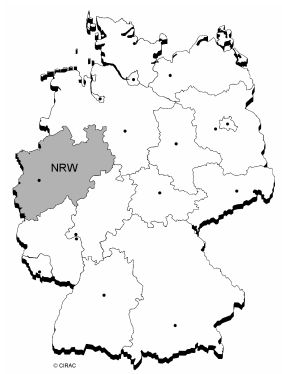

La Rhénanie du Nord-Westphalie (Nordrhein-Westfalen, NRW) est un poids lourd. Son PIB (501 milliards $€$ en 2006) la place avant les Pays-Bas voisins (528 milliards $€$ ). Avec un bon cinquième du PIB allemand $(22 \%)$, la NRW est le premier Etat d'Allemagne, avant la Bavière (près de $18 \%$ ) et le Bade-Wurtemberg (14,6\%) - voilà, en termes de richesse, le trio de tete des sites allemands. La Hesse, en quatrième position, ne 'pèse' que $9 \%$ dans le PIB de la RFA. Elle compte 18 millions d'habitants, soit un cinquième de la population allemande.

D'une superficie moyenne $\left(34000 \mathrm{~km}^{2}\right)$, soit un petit dixième du territoire allemand seulement, elle est très densément peuplée avec 530 habitants au $\mathrm{km}^{2}$, contre 230 pour la RFA. Mais cette moyenne masque de très fortes disparités territoriales : le nord-est du Land, par exemple, est très faiblement peuplé, alors que dans la seule conurbation de la Ruhr, le cœur industriel du Land, on recense 6 millions d'habitants, soit en moyenne 2200 habitants au $\mathrm{km}^{2}$. Dans la ville de Herne, non loin de Dortmund, la densité atteint son maximum : 3385 . On dénombre 11 villes de plus de 200000 habitants dans le Land, dont six de plus d'un demi million d'habitants: Bochum (579 000), Bottrop (589 000), Cologne (968 000), Duisburg (512 000), Düsseldorf (capitale de la NRW, 571 000) et Essen (592 000). Toutes sont situées dans la Ruhr, sauf Düsseldorf et Cologne.

Frontalière des Pays-Bas au Nord et de la Belgique à l'Ouest, la NRW se caractérise par une grande diversité régionale qui trouve son reflet jusque dans le nom du Land, créé après la guerre par la juxtaposition du nom de deux des trois provinces réunies dans ses frontières. Celles-ci correspondent schématiquement à autant de grands ensembles à la fois géographiques, culturels et économiques :

\footnotetext{
*Version revue et augmentée d'un article paru dans Regards sur l'économie allemande, nº 62, juillet 2003.
} 
- la Rhénanie à l'ouest, dont la culture (catholique) et l'économie sont déterminées par l'axe sudnord du Rhin et la proximité des ports d'Anvers et, surtout, de Rotterdam. Elle s'étend de Bonn à Duisburg jusqu'à la frontière hollandaise, en passant par Cologne et Düsseldorf. Son économie : commerce, finance, logistique, industrie ;

- au nord-est, la Westphalie, faiblement peuplée, et où se mêlent protestantisme (Gütersloh) et catholicisme (Münster, Paderborn). Dans cette région à dominante agricole émerge la ville de Gütersloh, fief des groupes Miele et Bertelsmann;

- au centre, orientée est-ouest (de Dortmund à Duisburg, où se rejoignent Ruhr et Rhin), la vallée de la Ruhr, très densément peuplée, industrieuse et protestante. C'est là le vieux bassin industriel (charbon, acier, chimie, textile) de la NRW qui se prolonge au sud dans la vallée de la Wupper, berceau historique de la métallurgie (Solingen), du textile et de la chimie (Bayer). Cette région est aujourd'hui fortement tertiarisée et orientée high-tech.

NRW : concentration des activités sur le Rhin et la Ruhr

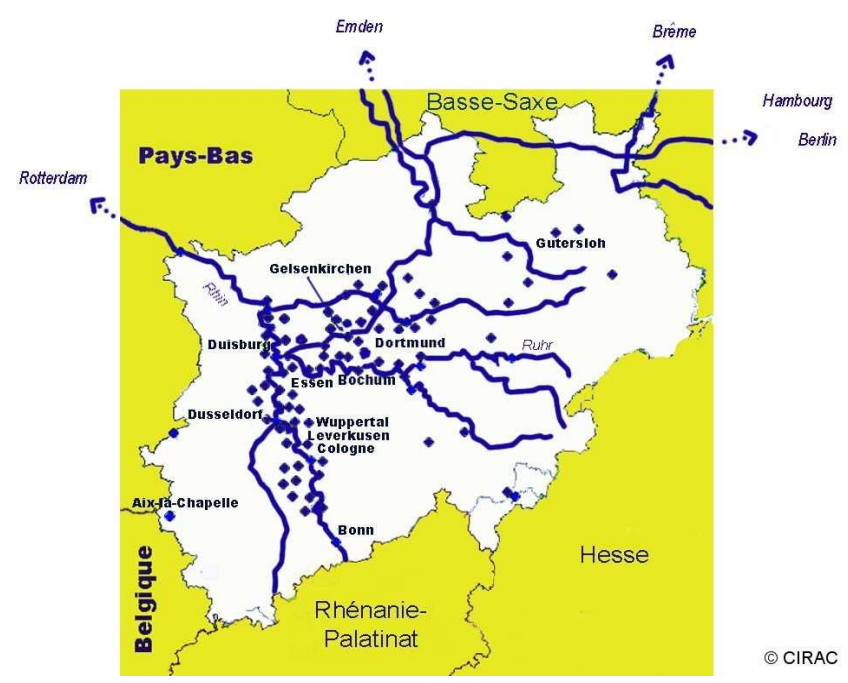

En termes de poids économique, d'infrastructures de production comme de recherche et de potentiel d'innovation, la Rhénanie du Nord-Westphalie présente de fortes similitudes avec la région Ile-deFrance. Düsseldorf, située sur le Rhin en amont du confluant Rhin-Ruhr, est l'un des principaux centres allemands de la finance et de l'assurance, et une importante plateforme logistique.

Cœur industriel de l'Allemagne d'avant la guerre, ce qu'il restait de son industrie lourde après 1945 fut d'abord démantelé avant d'être reconstruit et redynamisé, grâce notamment au Plan Marshall. Puis, après la crise du charbon et de l'acier qui s'amorce à la fin des années 1950, l'économie du Land s'est modernisée et diversifiée. A la suite du premier choc pétrolier, elle se tertiarise. Dans les années 1980, le Land mise sur une 'révolution' tertiaire un peu particulière à ses débuts : du charbon au show biz via les médias. La greffe a pris, et la tertiarisation trouve aujourd'hui ses prolongements dans les technologies de pointe. La structure par grands secteurs de l'économie du Land est désormais identique à celle de l'Allemagne : en 2005, les services y représentaient 70,1\% du PIB $(69,7 \%$ en moyenne allemande) et l'industrie au sens large 29,3\% (taux identiques). Si la NRW demeure un poids lourd industriel, elle n'est plus le site industriel allemand par excellence : ce rang revient désormais au Bade-Wurtemberg et à la Bavière. 
Aperçu sectoriel de l'industrie et des services en Rhénanie du Nord-Westphalie

\begin{tabular}{|c|c|c|c|c|c|c|}
\hline $\begin{array}{l}\text { Industrie } \\
(2005)\end{array}$ & $\begin{array}{c}\text { CA } \\
\text { en } m r d s \epsilon\end{array}$ & $\begin{array}{l}\text { Part } \\
\text { RFA }\end{array}$ & $\begin{array}{c}\text { Part des } \\
\text { export. alldes }\end{array}$ & $\begin{array}{l}\text { Services } \\
(2004)^{\text {c) }}\end{array}$ & $\begin{array}{c}\text { CA } \\
\text { en } m r d s \epsilon\end{array}$ & $\begin{array}{l}\text { Part } \\
\text { RFA }\end{array}$ \\
\hline Chimie & 51,1 & $35,9 \%$ & $25,3 \%$ & $\begin{array}{l}\text { Commerce et } \\
\text { service après-vente }\end{array}$ & 442,1 & $31,5 \%$ \\
\hline $\begin{array}{l}\text { Construction } \\
\text { mécanique }\end{array}$ & 39,2 & $23,1 \%$ & $20,1 \%$ & $\begin{array}{l}\text { Transport et } \\
\text { télécoms. }\end{array}$ & 121,9 & $49,0 \%$ \\
\hline $\begin{array}{l}\text { Prod. et transf. } \\
\text { des métaux }\end{array}$ & 35,8 & $51,8 \%$ & $48,4 \%{ }^{\mathbf{b})}$ & $\begin{array}{l}\text { Services aux } \\
\text { entreprises } \\
\text { Hôtellerie/ }\end{array}$ & 49,4 & $16,5 \%$ \\
\hline Automobile & 31,5 & $11,3 \%$ & $14,5 \%$ & gastronomie & 11,0 & $20,9 \%$ \\
\hline $\begin{array}{l}\text { Agro- } \\
\text { alimentaire }\end{array}$ & 29,2 & $22,2 \%$ & n.c. & $\begin{array}{l}\text { Culture, sports et } \\
\text { divert./loisirs }\end{array}$ & 10,9 & 25,2 \\
\hline Produits métall. & 27,9 & $34,8 \%$ & $48,4 \%^{\mathbf{b})}$ & $\begin{array}{l}\text { Informatique/ } \\
\text { bureautique }\end{array}$ & 9,6 & $21,0 \%$ \\
\hline Total industrie & $323^{a)}$ & $22,7 \%$ & $22,1 \%$ & Total services & 710,8 & $29,6 \%$ \\
\hline
\end{tabular}

Source des données : Jahreswirtschaftsbericht NRW 2007. a) chiffre arrondi ; b) les données sur les échanges ne distinguent pas entre ces deux catégories ; c) ce tableau ne contient que les catégories les plus importantes.

Entre Ruhr et Wupper : le berceau de la culture industrielle et sociale allemande

La région entre la Ruhr et la Wupper, de culture protestante, l'un des creusets historiques de la métallurgie et de la mécanique allemandes, fut le berceau de l'industrialisation de l'Allemagne. Un groupe comme Mannesmann en est l'une des entreprises emblématiques. En 1886, les frères Mannesmann, issus d'une famille de forgerons de Remscheid, et qui possèdent une manufacture réputée de limes, déposent un brevet fondamental pour l'avènement de l'ère industrielle : il porte sur un procédé permettant de produire des tubes d'acier sans soudure. Ce procédé a trouvé d'innombrables applications : dans l'ameublement (le fauteuil Bauhaus de Mies van der Rohe) ou l'urbanisme (des mâts des lampadaires aux conduites d'eau potable ou systèmes d'égouts), sans oublier les transports (vélocypèdes, pièces pour les moteurs automobiles, chaudières de locomotives ou de paquebots) ni la chimie (récipients) et l'industrie pétrolière (pipelines)...

Cette entreprise, comme tant d'autres dont l'essor remonte à l'époque bismarckienne, participe de cette culture d'engagement social qui a mené à la genèse du système allemand de protection sociale. Les Mannesmann, Krupp, Thyssen, Bayer, etc., créent dès la fin du XIX ${ }^{\mathrm{e}}$ siècle leurs propres caisses d'assurance maladie ou d'épargne retraite et vont jusqu'à construire des logements et des hôpitaux pour leurs salariés. Nombre de ces institutions existent toujours, comme les hôpitaux Krupp à Essen.

Indépendamment de ces entreprises se créent, dans le contexte de cette culture sociale, des caisses d'assurance maladie, comme la Barmer Ersatzkasse (BEK), née à Barmen, localité vouée au textile (passementerie), à la métallurgie et à la chimie comme sa jumelle d'aujourd'hui, Saint-Etienne, et qui fut intégrée dans la municipalité de Wuppertal lors de la constitution de celle-ci au tout début du XX $\mathrm{XX}^{\mathrm{e}}$ siècle. C'est à Barmen que Friedrich Bayer (1825-80) inventa notamment les colorants à l'aniline, avant de créer l'entreprise à laquelle il donna son nom, et dont le site est depuis à Leverkusen, au bord du Rhin, près de Cologne. C'est à Barmen également qu'était né Friedrich Engels.

Dans l'immédiat après-guerre, l'industrie lourde entre Ruhr et Wupper est le théâtre d'une coalition d'intérêts entre Alliés occidentaux et syndicats renaissants autour de la démocratisation de l'économie, et qui débouche, en 1951, sur la «Loi accordant aux salariés le droit de cogestion dans les conseils de surveillance et les comités directeurs des entreprises minières et sidérurgiques » (Montanmitbestimmungsge$s e t z$ ). Cette région est ainsi également le berceau historique de la cogestion au cœur du " modèle rhénan » et fief des traditions syndicales qu'incarne toujours, mais dans une version modernisée, un syndicat comme IG Metall. Il va sans dire que ces mêmes traditions sont au fondement de la social-démocratie dont la Rhénanie du Nord-Westphalie est pareillement l'un des berceaux, et qui a vu évoluer la doctrine du SPD lors du congrès de Bad Godesberg en 1959.

Contrairement à l'époque où le Land avait l'image d'un "pays noir », il présente aujourd'hui des atouts environnementaux, touristiques, indéniables et fortement valorisés. En qualité de vie, facteur d'importance stratégique pour une économie tertiaire, même le bassin de 
la Ruhr n'a plus rien à envier aux Länder du Sud. La NRW est même le leader allemand en termes d'offre culturelle, sportive et de loisirs, rassemblant un quart des entreprises $(24 \%$ en 2004) du secteur recensées en RFA et du chiffre d'affaires réalisé (25\%).

\section{Une industrie aujourd'hui tertiarisée}

En quarante ans, la Rhénanie du Nord-Westphalie a connu une rapide tertiarisation. La reconversion d'une économie à monoculture industrielle (charbon, sidérurgie, chimie) au sortir de la guerre, accélérée à partir des années 1980, s'est traduite par un recul de $28 \%$ de l'emploi industriel en vingt ans, sans toutefois aboutir à une désindustrialisation. Au contraire, le développement des services recouvre en réalité une rapide tertiarisation de l'industrie : l'emploi dans les services aux entreprises a crû de $85 \%$ durant la même période. C'est là le fruit d'une longue politique de reconversion qui a su mettre à profit à la fois les richesses existantes et le mouvement général des mutations structurelles : la localisation des entreprises industrielles s'effectuant au plus près des gisements de services, et les prestataires de services s'implantant à proximité des concentrations industrielles et des nœux de communication.

Car les services ne se sont pas constitués ex nihilo dans ce Land dont une ville (Bonn) fut la capitale de la RFA d'avant la réunification, accueillant entre autres le ministère fédéral des PTT dont sont issus au milieu des années 1990 la Deutsche Post AG et la Deutsche Telekom AG, et autour duquel s'était constitué au préalable un tissu d'activités liées soit à la logistique, soit aux télécommunications. Dans le seul segment du transport et des télécommunications, le Land réalise la moitié (49\% en 2004) du chiffre d'affaires de la République fédérale.

La Deutsche Bahn AG, privatisée depuis une bonne décennie, a maintenu son siège à Bonn ; cela contribue à expliquer pourquoi les activités de fret ferroviaire du Land représentent le tiers du total allemand (29,1 \% en 2005). Par ailleurs, les secteurs industriels dominants dans le Land sont non seulement largement tributaires du transport (ex. : automobile, chimie, métallurgie), mais sont également ceux qui ont développé les premiers une vision globale de la logistique (ex. l'automobile sous la pression des flux tendus). Petit à petit, les process de production se modernisant, les services liés à ces activités se sont constitués en grappe autour de ces pôles industriels, soit par essaimage, soit par création.

L'essor des activités liées à la logistique a bénéficié aussi d'un réseau de voies de communications fluviales unique en Europe, développé au fur et à mesure de l'industrialisation du Land. Deux grands axes fluviaux, le Rhin et la Ruhr, relient les ensembles que sont la Rhénanie et le bassin de la Ruhr, et débouchent en aval sur la Mer du Nord (port de Rotterdam). S'y ajoute un étroit maillage de canaux reliant ces deux axes naturels aux ports d'Anvers, de Hambourg et, via le Schleswig-Holstein, au port de Kiel sur la Baltique. Près de la moitié du fret fluvial allemand (46,3\% en 2005) passe ainsi par le Land. Le port fluvial de Duisburg (www.duisport.de), au premier rang mondial (49,7 millions de tonnes de fret transbordées en 2006), se trouve ainsi à l'interconnexion des liaisons intermodales entre l'Allemagne, la Belgique, la Grande-Bretagne, l'Irlande, les Pays-Bas et l'espace de la Mer Baltique. Il est actuellement en cours d'extension, sa situation au confluant Rhin/Ruhr le prédestinant à devenir une plateforme intégrée de logistique au cœur de l'Europe. Il offre ainsi par exemple, tous les jours ouvrables, une liaison ferroviaire automatisée (conteneurs et autres unités de transport intermodal) vers Vienne et Budapest. 
La Rhénanie du Nord-Westphalie dispose certes d'un avantage compétitif historique lié à sa position centrale dans le pentagone de compétitivité européen (la «banane bleue »). Mais son orientation d'axe ouest-est dans une Europe désormais élargie confère une valeur stratégique nouvelle aux liaisons autoroutières et ferroviaires reliant Paris à Varsovie via Aix-laChapelle, Cologne et Berlin. Cet axe, qui traverse notamment la conurbation de la Ruhr, et qui croise les grandes liaisons nord-sud, est intégré dans un réseau extrêmement dense d'infrastructures de communication. Avec 7 aéroports dont Bonn/Cologne (numéro un allemand et européen pour le trafic passager dans le segment low cost) et, surtout, Düsseldorf ( $3^{\mathrm{e}}$ d'Allemagne), le Land se situe en troisième position allemande en termes de trafic de passagers derrière la Bavière (aéroport de Munich, numéro 2) et la Hesse (Francfort, numéro 1), mais en seconde position après Francfort en ce qui concerne le fret aérien (693 millions de tonnes transbordées en 2005, soit 23,6\% du total allemand).

Si le réseau routier est dense, avec $200 \mathrm{~km}$ d'autoroutes et $30000 \mathrm{~km}$ de routes fédérales (l'équivalent de nos nationales), il n'en atteint pas moins aujourd'hui la limite de ses capacités, présentant des goulots d'étranglement chroniques aux principaux points d'interconnexion régionaux comme transeuropéens : périphérique de Cologne, échangeurs autoroutiers sur les axes Cologne/Francfort/Munich ou Aix-la Chapelle/Berlin... Quant au réseau de la Ruhr, emprunté par le transit transeuropéen, il est au moins aussi engorgé que celui de la région parisienne.

La nécessaire extension du réseau routier du Land s'était longtemps heurtée à plusieurs obstacles. En premier lieu le manque de moyens à une époque où la priorité de la politique allemande des transports était la mise à niveau du réseau est-allemand et qui s'explique plus généralement depuis par l'endettement des budgets publics du Bund comme du Land. En second lieu, les contradictions flagrantes entre les objectifs environnementaux et les impératifs d'une politique des transports à l'appui de la compétitivité territoriale d'un gouvernement de coalition SPD/Verts au pouvoir dans le Land de 1990 à 2005. Les Verts ont ainsi longtemps bloqué divers projets stratégiques du SPD, notamment l'extension du réseau autoroutier et la construction d'itinéraires de délestage qu'implique la densité du trafic (quelque 100000 véhicules par jour en moyenne sur les seules autoroutes). Il en fut de même de l'extension des aéroports internationaux de Düsseldorf et Bonn/Cologne, à la limite de leurs capacités, ou de celui de Münster/Osnabrück, plus au nord, à la frontière de la Basse-Saxe, voué aux liaisons interrégionales.

Aujourd'hui, le contexte est devenu plus favorable à une modernisation des infrastructures : celle des Länder de l'est étant achevée, la priorité de la politique allemande des transports va désormais à leur aménagement à l'ouest; dans une Europe à 27, la mise en œuvre accélérée du programme Réseaux trans-européens de transports (TEN-T) de l'UE est une urgence ; et, sous la houlette du ministre-président Jürgen Rüttgers (CDU), le nouveau gouvernement CDU/FDP élu au printemps 2005 en NRW, les impératifs de développement économiques reprennent le dessus dans la politique du Land. Si le gouvernement fédéral CDUCSU/SPD a décidé d'accroître la part des investissements consacrée aux infrastructures, suivi par les Länder, l'impératif de consolidation des finances publiques fédérales et régionales limite néanmoins l'effort budgétaire consenti. La NRW a ainsi programmé 1 milliard $€$ pour l'extension de son réseau routier d'ici... 2015.

La présence de Deutsche Telekom ou de l'ex-sidérurgiste Mannesmann auquel a succédé l'opérateur de télécommunications Vodafone (après son absorption en 2000, les activités sidérurgiques, dont les tubes d'acier sans soudure, ont été revendues, ces dernières à 
Vallourec), ont été un atout pour le développement des activités liées aux TIC dans le Land. D'autant qu'elles se sont cumulées avec une forte concentration de médias : le premier groupe de presse régionale allemand: Westdeutsche Allgemeine Zeitung (Essen), le groupe Bertelsmann (Gütersloh), la principale chaîne publique régionale allemande (Westdeutscher Rundfunk, dont le budget est équivalent à celui de France Télévisions), la radio nationale Deutschlandfunk et la radio internationale Deutsche Welle (toutes trois à Cologne). C'est d'ailleurs la municipalité de Cologne qui, la première, avait entrevu l'enjeu stratégique de dynamisation régionale que représentait l'audiovisuel lors de sa libéralisation au début des années 1980, et qui a attiré sur son site l'investisseur luxembourgeois CLT, partenaire historique du groupe Bertelsmann (fusionnés aujourd'hui dans RTL Group) dans la chaîne privée RTL. De proche en proche, la production s'est installée autour des diffuseurs, en attirant d'autres, transformant la NRW en l'un des deux principaux sites médiatiques avec la Bavière. Et, tous les ans, les professionnels de cette branche très polycentrique se rassemblent à Munich (Medientage München) en automne, à Cologne (Medienforum $N R W$ ) au printemps.

Dès la fin des années 1980, sous la houlette de Wolfgang Clement (SPD), alors ministre de l'économie, puis ministre-président, le Land avait officiellement placé sa politique de reconversion industrielle sous le signe du show-business. Cela a longtemps prêté à sourire, mais l'image porteuse des médias a fini par se répercuter sur l'ensemble des activités du Land. Aujourd'hui, alors que l'Allemagne est le premier marché médiatique européen, un petit tiers (28,8\% en 2004) de sa production audiovisuelle est réalisée en NRW. C'est à Hürth, près de Cologne, qu'ont été produites les versions allemandes de Big Brother. La ville de Duisburg a servi de décor, à partir de 1981, aux aventures télévisées du commissaire Schimansky. Quant au massif de l'Eifel, il a été célébré par les films Heimat d'Edgar Reitz. La NRW est également leader en matière de production musicale, ce qui explique par exemple que les chaînes musicales comme Viva s'y soient installées lors de leur création. Il abrite nombre de grandes manifestations comme le festival Rock am Ring (sur le circuit du Nürburgring), le plus important d'Allemagne. Cette évolution, qui a tiré profit en outre d'une offre culturelle très riche, a renforcé l'attractivité du Land et contribué à entretenir une logique d'innovation, étayée depuis le milieu des années 1980 par une politique délibérée de développement des TIC et de tous les services liés à l'économie du savoir.

$\mathrm{Au}$ cours de la dernière décennie du $\mathrm{XX}^{\mathrm{e}}$ siècle, la politique de reconversion du Land a changé d'orientation : il ne s'agissait plus tant de compenser les déficits de compétitivité par un soutien aux activités en déclin ou le lancement de grands programmes pour développer des secteurs nouveaux (comme ce fut le cas à l'origine de la politique volontariste d'un «saut technologique » : du charbon aux TIC). Mais il s'agissait au contraire de soutenir de manière ciblée le potentiel d'innovation et de compétitivité existant. Le soutien n'est donc plus ni territorial ni sectoriel, mais différencié et centré sur les activités ou pôles régionaux à fort potentiel. Cela se traduit par une politique d'offre visant la constitution et le grappage de ces clusters et passant par la mise en réseau des mécanismes d'information, des soutiens financiers, des programmes de formation, des acteurs économiques et des milieux de recherche. La politique de recherche à proprement parler s'inscrit dans cette politique structurelle globale dont elle est partie intégrante

La NRW, quoique longtemps gouvernée par une coalition SPD/Verts, a donc poursuivi la même approche politique que la Bavière chrétienne-démocrate, sa principale concurrente parmi les Länder. Le changement de gouvernement ne modifie pas fondamentalement cette 
approche. La nouvelle coalition prolonge les orientations des gouvernements Wolfgang Clement ou Peer Steinbrück, l'actuel ministre fédéral des Finances qui, avant d'être ministreprésident de NRW (2002/05) en fut le ministre de l'Economie, des PME, de la Technologie et des Transports (1998/2000) puis le ministre des Finances (2000/02). Les inflexions nouvelles qu'elle y apporte sont déterminées par un double impératif : l'effort accru d'innovation (technologies-clefs, développement des pôles de compétitivité, soutien aux PME...) et la consolidation des finances publiques (la dette du Land s'élevait à 115 milliards $€$ en 2006, soit un peu plus de $7600 €$ par habitant, ce qui place la NRW dans le haut de la fourchette des Länder moyennement endettés). C'est dans ce nouveau contexte, et à la faveur de la fin du Traité CECA, que le Land, le gouvernement fédéral et les partenaires sociaux ont conclu au début 2006 l'abandon définitif des activités d'extraction de houille en 2018.

Cette politique visant à créer des synergies cumulatives, menée en application de la Stratégie de Lisbonne et ouvertement dédiée aux services dans la mesure où, dans ce Land plus qu'ailleurs, ils s'interpénètrent largement avec une industrie hautement moderne, se décline de plusieurs manières.

Elle s'est manifestée d'abord, sous le gouvernement SPD/Verts, par la concentration des compétences administratives en la matière grâce à la création d'un 'super-ministère' de l'Economie et du Travail, en charge également des technologies et des qualifications. Le nouveau gouvernement CDU/FDP, tout en conservant une approche de synergies pour ses dix ministères, a reconfiguré leurs attributions pour mieux les adapter aux nouveaux objectifs. Questions économiques et sociales ont été séparées. Le ministère de l'Economie s'est vu adjoindre les PME et l'Energie, deux dossiers clefs actuels.

La politique structurelle du gouvernement Rüttgers prolonge l'orientation que lui avait donnée son prédécesseur en matière de soutien aux PME. La banque publique régionale West-LB, rebaptisée NRWBank en 2004, et transformée en l'équivalent régional allemand de la Caisse des Dépôts française, s'est vu à cette occasion confier pour mission particulière l'accompagnement des PME, principalement des 'gazelles' (crédits, capital-risque, etc.) S'il s'agit de dynamiser l'innovation, il importe aussi d'assurer la relève puisque ce sont les PME qui créent de l'emploi, alors que les grandes entreprises réduisent leurs effectifs. Certes, 21 des 50 plus grands groupes industriels allemands ont leur siège dans ce Land, dont Bayer, E.ON, Ford, Hochtief AG, Miele, RWE ou ThyssenKrupp. Dans le segment commerce/distribution, le Land en compte 22, d'Aldi à Rewe en passant par Karstadt Quelle. Mais le tissu des entreprises est constitué à plus de $99 \%$ de PME. En 2006, elles emploient $67,8 \%$ des salariés, forment $82 \%$ des apprentis du Land, et contribuent pour $42 \%$ à la création de richesse - ce qui coïncide avec la moyenne nationale allemande.

La NRW a également une lecture européenne de la reconversion régionale et du rôle des PME. Toujours dans le contexte de la Stratégie de Lisbonne, elle s'est associée à un réseau de coopération: Industrial Change Network (ICN), qui, depuis mai 2003, rassemble une vingtaine d'institutions régionales de soutien aux PME des Etats membres de l'UE. Ce réseau était né à l'initiative de l'association European Industrial Regions Association (EIRA), créée en 2002 par diverses régions de l'UE pour promouvoir « l'apprentissage tout au long de la vie » dans le domaine de la politique structurelle régionale. L'ICN, dont la réunion constitutive s'était tenue début juin 2003 à Ostrava en République tchèque, a pour objectif de mieux faire connaître à ses membres les best practices en matière de reconversion industrielle, d'innovation et de soutiens européens. En NRW, les programmes de ce 
réseau soutenu par l'UE et le Land sont coordonnés par le Bureau de Soutien aux activités industrielles et commerciales de l'artisanat : le Landes-Gewerbeförderungsstelle des NRWHandwerks (www.lgh.de).

\section{Mise en réseau des pôles d'innovation et de high tech}

Le cœur de la politique de reconversion consiste à mettre en réseau les pôles de dynamisme et d'innovation (clusters) de la Ruhr pour les relier en grappe. Elle associe tous les acteurs impliqués - des pouvoirs publics aux fédérations professionnelles en passant par les universités et centres de recherche et, bien sûr, les entreprises. Si cette approche est antérieure aux nouveaux objectifs de Lisbonne définis par la Commission et qui lient politiques d'innovation et de cohésion, elle n'en est pas moins largement inspirée dès l'amont, la NRW jouant un rôle très actif au sein du Comité des Régions qui doit fournir un rapport dressant l'état des lieux de sa mise en œuvre d'ici le Sommet de printemps 2008.

Il s'agit pour le Land de créer une dynamique cumulative favorable à la croissance et l'emploi alors qu'il n'a pas encore totalement achevé sa restructuration (certaines zones urbaines ont des taux de chômage frisant les $20 \%$ ), mais qui dispose d'un énorme potentiel dans les domaines suivants : logistique, sciences du vivant, technologies des microstructures et nanotechnologies, énergie, services liées aux TIC, ou encore design, textile-habillement et éco-industries.

\section{Un exemple de mise en réseau des compétences dans le design : Zeche Zollverein}

Cette politique crée un maillage de proche en proche : Düsseldorf abrite le premier salon professionnel allemand de la mode, l'igedo et ses multiples avatars (www.igedo.de). Le Land de NRW a donc lancé une Initiative Avenir Textile (Zukunftsinitiative Textil $N R W$ ) qui s'inscrit dans le cadre d'une modernisation de la branche à l'échelle de l'Allemagne et se décline, dans le Land, sous la forme d'une offensive à destination des textiles techniques (www.zitex.nrw.de). Or parallèlement, Essen est un haut lieu du design, avec l'Ecole Volkwang et l'Université.

Le lien entre ces trois éléments et leurs divers prolongements est constitué par une opération qui, à première vue, leur semble plutôt étrangère : la réhabilitation du patrimoine architectural industriel. En l'occurrence, il s'agit de la réhabilitation d'un haut lieu de l'histoire minière d'Essen : du puits avec sa cokerie, Zeche Zollverein, désaffecté depuis la fin des années 1960. L'opération, qui bénéficie de fonds structurels européens au titre de l'objectif 2 (reconversion structurelle des régions en difficulté) depuis février 2002 et est subventionnée à hauteur de 61 millions $€$, consiste en la transformation de ces installations de plus de 100 ha en un parc d'activités entièrement dédié au design : avec manifestations culturelles, foires-expositions (il accueille depuis 2005 un forum mondial de design et d'architecture, Metaform), plateformes de transfert et centres de formation au design au sens large (www.zollverein.de).

Cet effort collectif du réseau d'acteurs s'est soldé par des retombées en termes d'image. Au printemps 2006, le Zeche Zollverein a été de surcroît classé par 1'UNESCO parmi les sites du patrimoine culturel mondial et figure sur la Route européenne de la culture industrielle. La ville d'Essen, pour sa part, sera capitale culturelle européenne en 2010 .

L'approche en réseau se double de politiques sectorielles de R\&D comme de transfert technologique, l'accent étant désormais mis sur ce dernier point avec le développement de partenariats public-privé. En matière d'innovation et de hautes technologies, la NRW talonne la Bavière, un des leaders européens. On y recense 58 universités (dont les très réputées Friedrich-Wilhelms-Universität de Bonn et Rheinisch-Westfälisch Technische Hochschule : $R W T H$ ) d'Aix-la-Chapelle, mais aussi le siège de la Conférence permanente des Présidents d'Université), 35 centres non universitaires de recherche (dont le Max Planck-Institut für 
Mathematik, des instituts Fraunhofer, Helmholtz et Leibniz, le centre de recherche spatiales DLRG ou le centre de recherches interdisciplinaire FZJ de Jülich), quelque 60 plateformes technologiques et une trentaine de centres de transfert technologique.

Et pourtant, ce Land est en retard sur la Bavière et le Bade-Wurtemberg, où la R\&D dépasse l'objectif des $3 \%$ du PIB que s'étaient fixé les Etats membres à Lisbonne. Le nouveau gouvernement s'est donc fixé pour objectif de porter à au moins $3 \%$ du PIB d'ici 2015 l'effort de R\&D. Pour ce faire, il mène sa politique d'innovation sur deux fronts. D'une part, dans la continuité de la politique précédente, les programmes de transfert technologiques et de recherche incrémentale directement liés à la modernisation des activités industrielles seront poursuivis. Mais un effort particulier sera consenti aux activités les plus porteuses sur lesquelles se concentrent désormais les aides ou incitations financières dont l'attribution se veut aussi plus sélective que par le passé. En outre, la politique d'innovation suit désormais une approche globale, intégrant recherche, marché et, surtout, la formation en amont. C'est dans ces réorientations que consiste principalement la différence avec l'action du gouvernement précédent qui avait mené une politique d'incitation généreuse mais somme toute peu ciblée ; maintenant que le terrain est labouré et qu'une solide culture de l'innovation a germé dans le Land, il s'agit de permettre à ces pôles d'excellence de se développer.

C'est ainsi que le Land a adopté une Loi sur la Liberté des Universités (Hochschulfreiheitsgesetz), entrée en vigueur le $1^{\mathrm{er}}$ janvier 2007, qui accorde une large autonomie aux établissements d'enseignement supérieur (universités et Fachhochschulen) : ils ont désormais le statut de collectivités de droit public (le rôle du ministère se limite donc au contrôle juridique), et sont de ce fait seuls compétents en matière de choix budgétaires, d'organisation et de GRH. Les établissements se contentent maintenant de signer des contrats d'objectifs quadriennaux avec les pouvoirs publics afin de s'insérer dans la politique globale d'innovation menée par le Land. Leur nouveau statut leur donne toute latitude pour conclure des partenariats avec les entreprises ou pour créer des plateformes de transfert. Enfin, la réforme leur permet de prélever dorénavant des frais de scolarité (dans la limite d'une enveloppe régionale plafonnée à 320 millions $€$ ) ; en contrepartie, ils s'engagent à accroître l'efficience de leurs enseignements.

Dans le cadre de sa politique d'innovation, le nouveau gouvernement conjugue d'une manière plus systématique que son prédécesseur l'utilisation des fonds structurels européens (Objectif 2) dont bénéficie la Ruhr au titre de sa reconversion avec les objectifs de la Stratégie de Lisbonne, accordant une large place au partenariat public-privé. C'est ainsi, par exemple que la Ruhr-Universität de Bochum et le groupe ThyssenKrupp AG ont décidé conjointement à la fin 2006 la création d'un institut de recherche dans le domaine des matériaux (ICAMS), implanté auprès de l'université, et doté d'un budget de 24 millions $€$ (moitié public, moitié privé) sur 2008/12, auxquels s'ajouteront des financements sur projet d'un même montant. Interdisciplinaire, l'institut associera recherche fondamentale et appliquée. Du côté public, il rassemble l'université de Bochum, l'Institut Max-Planck de recherche sur les matériaux ferreux (Düsseldorf), les centres $F Z J$ de Jülich et $R W T H$ d'Aix-la-Chapelle. Du côté privé, aux côtés de ThyssenKrupp, il réunit au sein d'un consortium : Bayer MaterialScience AG, Salzgitter AG et la Robert Bosch GmbH.

Le second front est celui du développement de grands programmes de R\&D dans les technologies clefs ou transversales, notamment celles définies dans le $7^{\mathrm{e}}$ PCRD de l'UE. Conformément aux objectifs européens, mais aussi conformément au mode de fonctionnement du 
système d'innovation allemand, ils mêlent eux aussi étroitement recherche fondamentale et appliquée, accordant de même une large place au partenariat public-privé afin de rendre le transfert technologique plus performant et la recherche fondamentale plus compétitive. Parmi les technologies-clefs prioritaires en NRW figurent ainsi les nanotechnologies/nouveaux matériaux, comme le montre la création de l'ICAMS, de même que les technologies de production. Mais l'effort principal se concentre aujourd'hui sur deux champs où le Land a un fort potentiel. D'un côté, dans les biotechnologies et technologies médicales : il dispose de plusieurs CHU réputés, dont l'Universitätsklinikum d'Essen, lié à la Medizinische Fakultät Duisburg-Essen (cancérologie, transplantation d'organes) ou l'Universitätsklinikum Aachen (neurologie, microet nanosystèmes). De l'autre, les technologies liées à l'énergie, et notamment au nucléaire.

Dans le domaine des biotechnologies, la NRW figure déjà parmi les régions allemandes les plus compétitives (biotechnologies médicales et « vertes »). Pour hisser le Land au rang des leaders européens, le gouvernement a prévu le lancement d'une Offensive dénommée BIO.NRW. Le montant des investissements s'élèvera à 100 millions $€$. Dans ce cadre s'inscrit par exemple la création à Dortmund d'une plateforme Max Planck de biotechnologie des systèmes (génomique, protéomique), financée à hauteur de 37 millions $€$, ou l'ouverture à Cologne d'un institut Max Planck de biologie moléculaire. A relever l'inauguration, en mars 2007, du Science-to-Business Center für Biotechnologie (S2B-Center Bio) à Marl : dédié aux biotechnologies «blanches » (produits et procédés industriels dans la chimie), il est financé principalement par le chimiste Degussa à hauteur de 50 millions $€$, le Land apportant un complément de 11,3 millions $€$ (période 2007/11).

Cette coopération s'inscrit dans le cadre plus large du développement de ce segment des biotechnologies, piloté par le NRW-Konsortium Weisse Biotechnologie qui associe, aux côtés de Degussa, d'autres groupes comme Bayer, Henkel, Lanxess et Cognis, ainsi que plusieurs PME et centres de recherche. Le consortium a posé sa candidature au concours des pôles de compétitivité BioIndustry 2021 que vient de lancer le Land. Le développement de ce soussecteur des biotechnologies est stratégique, la NRW totalisant un dixième des emplois européens dans la chimie, et cette industrie jouant un rôle sociétal indéniable dans le Land, comme le révèle par exemple l'implication d'un groupe comme Bayer (inventeur de l' « Aspirine ») dans la vie sociale et sportive (football) de Leverkusen.

Dans le domaine de l'énergie (comme dans celui de la recherche sur le génome ou les OGM), le nouveau gouvernement de NRW mise résolument sur " une politique libérée des contraintes idéologiques ». Non seulement il s'agit de préserver la compétence du Land (et de l'Allemagne) en matière de recherche sur le nucléaire, dont la sécurité des réacteurs, mais aussi de la dynamiser en l'inscrivant dans une politique plus globale de recherche en matière d'énergies renouvelables et de protection de l'environnement. La recherche se concentre donc sur les deux pôles d'excellence préexistants : le centre de Jülich et la RTWH d'Aix-laChapelle. Le partenariat public-privé est plus que jamais de règle : l'électricien E.ON a décidé de localiser à Aix-la-Chapelle le cœur de ses activités de R\&D ; à cet effet, il co-finance la création d'un institut de recherche sur l'énergie auprès de la RTWH à hauteur de 40 millions $€$, l'université apportant 30 millions $€$ de son côté.

Ces politiques suivent toutes la même logique de réseau que celle du précédent gouvernement : l'ensemble de la chaîne de création de valeur est concerné, de la recherche à la production en passant par les services, les champs de R\&D prioritaires correspondant à des secteurs d'activité présentant le plus fort potentiel dans l'économie régionale. Les outils en 
sont les prolongements des diverses politiques visant à développer l'ensemble des activités : soutien aux PME, à la création d'entreprise, concours d'innovation, etc. Mais le nouveau gouvernement apporte à cette stratégie une inflexion de taille pour tenir son objectif d'excellence dans la compétition territoriale allemande.

Le programme présenté le $1^{\text {er }}$ février 2006 par le ministre de l'Innovation, de la Science, de la Recherche et de la Technologie, Andreas Pinkwart (FDP), professeur d'économie à l'Université de Siegen et spécialiste notamment des PME, également vice-ministre-président $\mathrm{du}$ Land est parlant. La globalisation des activités a foncièrement modifié la donne : " ce n'est plus comme naguère, où la présence d'une université ou d'un centre de recherche était perçue automatiquement comme un avantage compétitif pour le site, amenant tout naturellement l'industrie intensive en R\&D à s'implanter au plus près ... C'est seulement si nos universités et centres de recherche parviennent à tenir leur rang dans la compétition mondiale que la proximité avec leurs usagers se transformera en avantage compétitif ».

\section{Quand le football entretient lien social et culture de la compétitivité}

Le groupe Bayer, né en 1863 et installé depuis 1912 à Leverkusen dont il a littéralement 'fait' le développement, est indissociable de l'histoire industrielle et culturelle de la région : il appartient à ces entreprises qui ont depuis toujours largement contribué à la cohésion sociale. L'engagement culturel et sportif (mécénat et sponsoring) de Bayer trouve là ses origines. Si le groupe finance 29 associations sportives (de la pêche à la ligne au tir sportif en passant par l'équitation), il sponsorise aussi le club de football Bayer 04 Leverkusen, né en 1904 sous la forme d'une association ouvrière de Bayer.

Entre Rhin et Ruhr, le football est roi depuis plus d'un siècle. On n'y compte pas moins de 6 clubs de la Bundesliga : Bayer 04, Borussia Dortmund, Borussia Möchengladbach, FC Schalke 04 (Gelsenkirchen), VfL Bochum ; il faut y ajouter DSC Arminia, bien qu'il soit situé à Bielefeld, c'est-à-dire en Westphalie. Car la vraie patrie du football allemand reste la Ruhr, avec sa culture minière, fondée sur la performance collective, telle qu'on peut la trouver, en France aussi, à Saint-Etienne ou dans le Nord. Signe de la modernité de cette culture : pour valoriser sa nouvelle identité, le groupe E.ON, né de la fusion Veba/Viag, a pris sous contrat en 2000 le club Borussia Dortmund.

Le club qui incarne le plus la fonction de lien social, de compétitivité et de culture de l'identité régionale qui accompagne le football est FC Schalke 04, créé lui aussi en 1904. Son sponsor principal fut longtemps l'assureur Victoria (né en 1853), aujourd'hui relayé par Gazprom. En 2001, le club a inauguré son nouveau stade (Veltins Arena), conçu pour recevoir plus de 60000 spectateurs et accueillir, outre des manifestations sportives, de grands concerts de variétés, de rock, et même des opéras (un Carmen en 2003). Il héberge aujourd'hui également le musée du club.

Ce stade est sponsorisé par le brasseur Veltins, dont le siège est à Grevestein dans le massif du Sauerland (au sud de la Ruhr) depuis sa création en 1824. Car la région est également le pays de la bière, avec d'innombrables appellations locales, mais aussi la marque la plus vendue en Allemagne : Krombacher Pils, produite par une entreprise familiale du même nom, sise dans le sud de la Westphalie. L'entreprise est l'un des principaux sponsors du sport professionnel en Allemagne (retransmissions TV de football, biathlon, Formule 1) et en Europe (athlétisme).

Dans ce contexte, une région au sein du Land a fonction de modèle pour le développement désormais plus ciblé des clusters : l'Euregio Meuse/Rhin qui s'étend de Liège à Hasselt, Maastricht, Heerlen et Aix-la-Chapelle. Dans ce triangle belgo-germano-hollandais dont le PIB dépasse de loin les 70 milliards $€$, les universités et établissements d'enseignement supérieur des trois régions limitrophes se sont constitués en réseau, associant, du côté allemand, notamment le centre de recherche interdisciplinaire de Jülich et la RTWH. Dans le cadre de la nouvelle politique d'excellence universitaire menée par le gouvernement fédéral, cette université est pour l'instant la seule du Land à s'être vu décerner le label national d'université d'élite. Autour de ces centres et établissements se sont greffées des plateformes 
de transfert de technologies, des pépinières d'entreprises et un dense réseau de PME innovantes nées par essaimage.

Ce cluster est considéré comme un " joyau » par l'institut de recherche économique RWI d'Essen (l'un des six grands instituts de conjoncture) qui avait réalisé en 2006 une étude sur la compétitivité de la NRW (Innovationsbericht 2006) pour le compte du ministère de l'Innovation. Aujourd'hui, le Land mise résolument sur la dynamique propre des pôles d'excellence de ce type pour combler son déficit en matière de technologies de pointe face à la Bavière ou le Bade-Wurtemberg et se hisser au premier rang des économies régionales les plus innovantes et compétitives. La fin programmée des subventions au charbon (quelque 750 millions $€$ par an pour le budget du Land) donnera aussi une plus large latitude au gouvernement pour parallèlement achever la restructuration des activités tout en menant l'indispensable consolidation budgétaire.

L'IMAGe de LA RHÉNANIE dU NORd-WeSTPHALIE S'EST TRANSFORMÉE. La représentation d'un "pays noir» sur le déclin, qui remonte aux premières crises du charbon et de l'acier dans les années 1950 et 1960, a cédé le pas à la réalité actuelle d'une économie régionale largement vouée aux services, industriels ou non, et plus encore à l'innovation, principalement dans les technologies transversales : celles dont on attend un effet levier sur l'ensemble des activités. La dynamisation du site NRW passe aussi par une politique d'échanges active, qu'elle soit européenne ou internationale, et dont les outils sont mis à la disposition des acteurs intéressés via le portail régional destiné aux investisseurs (www.gfw-nrw.de), mais aussi via notamment le portail fédéral www.ixpos.de, dédié au commerce extérieur. Car si la tertiarisation de l'économie s'est faite avec succès, elle s'est accompagnée d'un recul des parts de marché à l'export : la part du Land dans les exportations allemandes ne représente que $17 \%$ en 2006. C'est là, paradoxalement, la contrepartie d'une industrie hautement compétitive dans des branches fortement internationalisées, et donc plus fragiles aux chocs extérieurs. L'entrée en vigueur, ces prochaines années, de la Directive européenne relative aux services, en permettant la libre circulation des services au sein de l'UE, devrait bénéficier largement à une économie régionale construite aujourd'hui sur la logistique et les TIC.

\section{Indications bibliographiques}

Bosch G., « Struktur- und Beschäftigungspolitik für das Ruhrgebiet », conférence tenue le 23-02-2006 dans le cadre du Congrès ,Quo vadis, Arbeitsmarkt im Ruhrgebiet ?' (www.iatg.de)

BOURGEOIS I., «Mannesmann AG : de la sidérurgie à la 'nouvelle économie' », in BOURGEOIS I. (dir.), Allemagne 2001. Regards sur une économie en mutation, Cirac, Levallois, 2001

BOURGEOIS I., «Site Allemagne : une industrie de services », Regards sur l'économie allemande, $n^{\circ} 58$, octobre 2002

«Innovationsstandort Nordrhein-Westfalen », supplément au quotidien Frankfurter Allgemeine Zeitung, 01$10-2002$

Jahreswirtschaftsbericht NRW. Bilanzen, Fakten, Perspektiven 2003, Ministerium für Wirtschaft und Arbeit des Landes Nordrhein-Westfalen (www.mwa.nrw.de)

Wirtschaft in NRW 2007. Konjunktur, Prognosen, Perspektiven, Ministerium für Wirtschaft, Mittelstand und Energie des Landes Nordrhein-Westfalen (www.wirtschaft.nrw.de)

RWI, Innovationsbericht 2006. Zur Leistungsfähigkeit des Landes Nordrhein-Westfalen in Wissenschaft, Forschung und Technologie. Forschungsprojekt für das Ministerium für Innovation, Wissenschaft, Forschung und Technologie des Landes NRW, Essen, 2006 (www.rwi-essen.de)

www.nrw.de et www.gfw-nrw.de. 


\title{
Bavière : la patiente construction d'une économie performante
}

\author{
Isabelle Bourgeois
}

La Bavière, poids lourd industriel allemand, est aussi l'un des principaux sites high-tech européens. II n'en a pas toujours été ainsi : au sortir de la guerre, le Land était une région deshéritée, coupée de ses anciennes zones d'échanges orientales par le Rideau de fer, et dont les ressources se limitaient à l'agriculture. Le 'miracle économique' bavarois est le fruit d'une politique de reconversion économique et de développement structurel régional menée avec conséquence pendant plusieurs décennies et favorisée par une rare continuité politique. A une phase d'industrialisation rapide a succédé la conversion du site industriel en un pôle d'excellence tertiaire, puis le développement des secteurs high-tech et des services innovants. Aujourd'hui, la priorité va à la mise en réseau des pôles de compétitivité constitués au fil du temps afin de décupler le potentiel d'excellence présent et futur. Depuis toujours dotée d'une très forte identité, la Bavière marie résolument tradition et progrès technologique. A la fin des années 1990, alors que fleurissaient les formules médiatiques, le Land avait même forgé un slogan pour traduire cette politique: "l'alliance entre l'ordinateur portable et la culotte de peau ». S'il peut prêter à sourire aujourd'hui, il n'en exprime pas moins la recette de l'excellence bavaroise. *

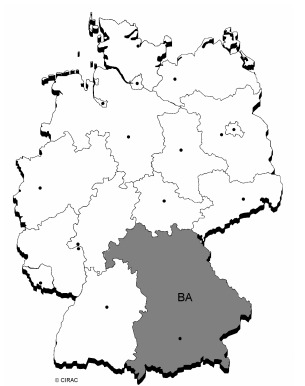

Avec un PIB de plus de 400 milliards $€$ en 2005, la Bavière est le second Land allemand et la $8^{\mathrm{e}}$ économie de l'UE 25. De loin le plus étendu d'Allemagne $\left(70552 \mathrm{~km}^{2}\right)$, il est de taille comparable à la République tchèque limitrophe à l'est. En nombre d'habitants (12,5 millions), il vient en seconde position parmi les Länder et en $9^{\mathrm{e}}$ position dans l'UE 27, après les Pays-Bas et juste avant la Grèce. La Bavière mène une politique européenne très active, notamment au sein du Comité des régions, né en 1994 comme expression de la subsidiarité des collectivités territoriales dans l'UE.

Dans le paysage allemand, la Bavière se distingue par une particularité : elle est la seule région d'Allemagne à avoir préservé son intégrité territoriale à l'échelle d'un millénaire, et elle dispose d'une plus grande autonomie étatique que les autres. L'Etat libre de Bavière (Freistaat Bayern), doté d'une Constitution propre dès 1946, n'avait accepté d'opérer que certains des transferts de souveraineté nécessaires à son intégration dans la RFA (1949). Il a depuis sa propre Cour constitutionnelle (Bayerischer Verfassungsgerichtshof) qui coexiste avec le Tribunal constitutionnel fédéral de Karlsruhe, et ses propres mécanismes démocratiques (recours au plébiscite, parti politique régional : CSU). Quant aux communes, elles y ont un poids politique décisif.

\footnotetext{
*Version revue et augmentée d'un article paru dans Regards sur l'économie allemande, n 40, mars 2000.
} 
La Bavière - chiffres-clefs 2005

\begin{tabular}{|lcc|}
\hline & Bavière & Allemagne \\
Superficie (en km2) & 70552 & 357093 \\
Habitants (en millions) & 12,5 & 82,4 \\
Densité de la population & 177 & 231 \\
PIB & 403,7 & 2245,0 \\
Part de la R\&D dans le PIB (en \%) a) & 3,0 & 2,5 \\
Nombre de brevets (pour 100 000 habitants) & 110 & 59 \\
Part des secteurs dans la création de richesse : & & \\
- Agriculture et sylviculture (en \%) & 1,0 & 0,9 \\
• Industrie (en \%) & 31,0 & 29,7 \\
- Secteur tertiaire (en \%) & 68,0 & 69,4 \\
Exportations (en milliards $€$ ) & 118,0 & 773,4 \\
Importations (en milliards $€$ ) & 95,1 & 581,8 \\
Taux d'exportation de l'industrie (en \%) & 44,9 & 39,6 \\
Actifs (en millions) & 5,9 & 36,6 \\
Taux d'activité (en \% de la population totale) & 47,6 & 44,3 \\
Part des travailleurs indépendants (en \% des actifs) & 12,2 & 11,2 \\
Taux de chômage (\%) b) & 7,8 & 11,7 \\
Taux de chômage des moins de 25 ans (en \%) & 8,7 & - \\
Nombre de faillites (sur 10 000 entreprises) & 82 & 125 \\
\hline
\end{tabular}

Sources des données : ministère de l'Economie, de l'Infrastructure, des Transports et des Technologies de la Bavière (état : août 2006 ; www.stmwirt.bayern.de/pdf/wirtschaft/Bayerns_Wirtschaft_in_Zahlen_dt.pdf), sauf Part des secteurs dans la création de richesse en RFA (www.invest-in-hessen.de) et Exportations, importations, taux d'exportation de l'industrie (données 2004 : ministère de l'Economie, de l'Infrastructure, des Transports et des Technologies de la Bavière, Wirtschaftsstandort Bayern. Kraftzentrum in der Mitte Europas, septembre 2005. a) en 2001 ; b) données administratives.

Le parti chrétien-social CSU est au gouvernement pratiquement depuis la fin de la guerre, et dispose de la majorité absolue depuis 1962. L'action politique de l'actuel ministre-président Edmund Stoiber, régulièrement réélu depuis 1993, et qui fut candidat au poste de chancelier fédéral lors des élections du Bundestag de 2002 (où Gerhard Schröder, SPD, l'emporta à une très courte majorité), poursuit donc les orientations définies au fil du temps par ses prédécesseurs. Parmi ceux-ci figura Franz Josef Strauss, chef du gouvernement de la Bavière de 1978 à 1988 et qui fut aussi, entre autres, ministre fédéral des Finances durant la première grande coalition (1966-69). Leur action avait permis de générer un environnement favorable à l'activité et à l'emploi. Cette stabilité politique a été déterminante pour le dynamisme économique du Land dans la mesure où elle a favorisé la conjugaison des intérêts des pouvoirs publics comme ceux des acteurs de l'économie et de la société, produisant sur la dynamique de croissance des effets cumulatifs qui s'auto-entretiennent. L'actuelle politique du gouvernement, et notamment son Allianz Bayern Innovativ lancée en 2006, se contentent donc d'entretenir un processus favorable à cet "écosystème de la croissance », pour reprendre une formule qui avait été forgée pour définir une possible politique d'innovation française (Blanc, 2004), mais qui caractérise à merveille les processus à l'œuvre dans cet Etat allemand.

\section{Une économie où services et industrie sont étroitement liés}

Comme le Bade-Wurtemberg limitrophe à l'ouest, la Bavière est un poids lourd industriel, largement ouvert à l'international. Comme dans le cas du Land voisin, la part prépondérante des services dans l'économie bavaroise (68\% des emplois) est trompeuse : plus de la moitié 
des activités du tertiaire est étroitement déterminée par la demande industrielle. Les biens industriels 'typiquement bavarois' ne se résument en effet pas à des véhicules industriels, fussent-ils produits par MAN AG à Munich, ou à des installations industrielles high-tech comprenant les roulements de FAG Kugelfischer AG \& Co. KG (Schweinfurt), comme l'explique un bilan de l'industrie bavaroise réalisé par l'institut ifo de Munich pour le compte du gouvernement bavarois (Industriebericht Bayern 2006). Ils sont bien plus que cela : "des solutions proposées pour des besoins spécifiques clairement définis », à l'instar de ces "total building solutions » que propose par exemple Siemens AG (Munich). L'industrie bavaroise est une industrie de services : non seulement les biens sont vendus accompagnés des prestations liées (financement, entretien, formation, etc.), mais leur production même requiert un nombre croissant de services, que ceux-ci aient été externalisés au fil de la restructuration des entreprises (comptabilité, $\mathrm{CAO}, \mathrm{GRH}$, etc.) ou que ceux-ci soient venus s'adjoindre par grappage au fur et à mesure de l'évolution des process de production (logistique, par exemple). C'est ainsi que les secteurs industrie et services sont interdépendants aujourd'hui, et que la demande industrielle entretient une offre croissante de services.

La spécificité bavaroise dans cette tendance globale à la tertiarisation qui se traduit généralement par un recul de l'emploi industriel est que, à la différence du reste de l'Allemagne, ce recul a presque entièrement pu être compensé par la création d'emplois tertiaires. Alors qu'en moyenne allemande, un tiers seulement des emplois industriels détruits depuis 1992 ont pu ainsi être compensés (en équivalent temps plein), cette part atteint $90 \%$ en Bavière selon l'institut ifo qui, dès lors, estime que la Bavière a mieux géré que les autres Länder la transition de l'ère industrielle à l'ère tertiaire. C'est ainsi que la Bavière présente, après le Bade-Wurtemberg, l'un des taux de chômage les plus bas d'Allemagne.

\begin{tabular}{|l|l|lr|}
\hline \multicolumn{4}{|c|}{ La structure des services en Bavière en 2005 (part de l'emploi) } \\
\hline Prestataires publics et privés & $40,7 \%$ & Transports et communications & $7,1 \%$ \\
Commerce, dont entretien biens de consommation & $22,0 \%$ & Hôtellerie, gastronomie & $6,8 \%$ \\
Immobilier, services aux entreprises & $18,2 \%$ & Banques et assurances & $5,2 \%$ \\
\hline
\end{tabular}

Source des données : Bayerisches Staatsministerium für Wirtschaft, Infrastruktur, Verkehr und Technologie, en coopération avec l'institut ifo, Industriebericht Bayern 2006, 10/2006.

Plus concrètement, les activités qui dominent dans le tertiaire sont donc le conseil (fiscal, juridique, ingénierie), l'audit et l'expertise, la $R \& D$, la publicité ou le leasing. L'ingénierie informatique s'y taille une place de choix grâce à la présence du concepteur de logiciels SAP AG (Walldorf). Deux autres catégories de services ont une place particulière dans l'économie bavaroise : la finance-assurance et les médias.

La Bavière accueille en effet la troisième place boursière allemande (Munich), spécialisée dans le Mittelstand ; et sa capitale est la seconde place bancaire allemande après Francfort : elle est le siège notamment de la HVB Group (membre de UniCredit Group), ex-HypoVereinsbank, née en 1998 de la fusion de la Bayerische Hypotheken- und Wechselbank et de la Bayerische Vereinsbank. La Bavière est également le premier site allemand de l'assurance, avec DAS (Munich), numéro un européen de l'assistance juridique, le groupe Allianz, la HUK-Coburg (second assureur automobile allemand, Coburg) ou le réassureur Münchner Rück (Munich Re Group), créé à Munich en 1880 et dont l'une des premières grandes opérations fut la gestion du tremblement de terre de San Francisco en 1906. La Bavière est en effet un des berceaux historiques de la finance : en 1486, les Fugger ouvraient la première 
banque à Augsburg, sur les routes de la soie, du sel et des épices. En 1540 se créaient les premières bourses, à Augsburg et Nuremberg, autre plate-forme d'échanges (ces villes hébergent aujourd'hui d'importantes foires-expositions).

La Bavière est aussi le principal site médiatique allemand avec la Rhénanie du NordWestphalie, sa concurrente : si cette dernière est le fief du groupe Bertelsmann, l'Etat libre est celui de ProSiebenSat.1 Media AG, l'ex-groupe Kirch. Les activités audiovisuelles se concentrent dans la région de Munich ( 8600 salariés), où se trouvent 20 radios (dont la radio privée Antenne Bayern) et 30 télévisions dont celles du groupe Kirch, l'établissement public Bayerischer Rundfunk. (radio et TV), l'offre du bouquet satellite numérique par abonnement Premiere, ou la chaîne de télé-achat HSE24. Les groupes Discovery Networks et Sony Pictures TV International ont récemment décidé d'implanter à Munich les studios de diverses chaînes. Dans la capitale se situe également le siège du câblo-opérateur Kabel Deutschland (premier d'Allemagne), et la filiale allemande de l'opérateur satellite SES, l'Astra Deutschland $\mathrm{GmbH}$, qui a transféré en 2006 son siège d'Eschborn (près de Francfort) à Unterföhring (près de Munich) pour y travailler en étroite collaboration avec la société Digital Playout Center $\mathrm{GmbH}$ au développement de l'offre numérique. C'est dans la région de la capitale aussi que se concentre la production audiovisuelle et cinématographique (les studios de la Bavaria Film sont leader en Europe), en faisant l'autre grand centre allemand avec Cologne. On y dénombre près de 1200 sociétés de production, avec un effectif de 9000 salariés (soit $23 \%$ du total allemand).

Mais le secteur inclut aussi la presse et l'édition. Munich est, après New York, le second site mondial du livre : les 234 maisons d'édition, dont Langenscheidt (dictionnaires) ou C.H. Beck (ouvrages spécialisés, notamment en droit, avec une collection au format de poche) publient chaque année 15000 titres. Dans le domaine de la presse, on trouve à Munich la rédaction de l'un des principaux quotidiens suprarégionaux allemands, la Süddeutsche Zeitung, ou encore le siège des éditions Burda (de la mode à l'information, avec l'hebdomadaire Focus, lancé en 1992 en concurrence frontale avec Der Spiegel, édité à Hambourg).

\section{Une industrie très diversifiée et omniprésente}

La préservation d'une base industrielle compétitive reste la priorité des politiques structurelles du Land; elle est la condition sine qua non pour un tertiaire florissant. Si cette base a dû être construite patiemment au sortir de la guerre, elle est aujourd'hui solidement ancrée 'dans le terroir' : contrairement à ce que peuvent laisser penser des atouts touristiques mondialement réputés et un environnement naturel savamment préservé, les activités industrielles sont omniprésentes.

Elles le sont à plusieurs égards. D'abord, la Bavière est le seul Land allemand à offrir la quasi-totalité de l'éventail sectoriel de la production manufacturière. Ensuite, les activités sont réparties de manière relativement équilibrée sur l'ensemble du territoire (10\% de l'emploi dans chacun des sept districts administratifs), même si on peut distinguer quelques prédominances régionales (un tiers des actifs de l'industrie se concentrent par exemple dans le district de Haute Bavière autour de la capitale : Munich). Et, si les agglomérations de $\mathrm{Mu}-$ nich ou de Nuremberg-Fürth-Erlangen dominent par définition, près de $70 \%$ des 2056 communes de Bavière peuvent être considérées comme des sites industriels. 
Enfin, si les grands groupes de réputation internationale (de BMW à Siemens en passant par Babcock Noell ou UPM Kymmene) sont les plus connus, le tissu des entreprises industrielles est composé aux deux tiers de PME (entreprises de moins de 500 salariés) : l'entreprise industrielle moyenne de Bavière occupe 150 salariés et réalise un CA d'un peu plus de 36 millions $€$. Le maillage des activités industrielles est donc très dense, qu'il s'agisse de la structure des entreprises ou de leur répartition régionale.

Ce maillage reflète une autre particularité bavaroise : la part prépondérante de l'artisanat au sein du Mittelstand. Un cinquième des entreprises artisanales allemandes se concentre en Bavière. Les 176000 entreprises artisanales recensées occupent en moyenne près de 5 salariés, ce qui contribue à la densité du tissu économique sur le territoire. Or plus des deux tiers d'entre elles $(68 \%)$ oeuvrent dans le secteur industriel ou celui des services aux entreprises. Du fait qu'elles sont des acteurs majeurs de la formation, elles apportent aussi une contribution fondamentale à la qualité des ressources humaines : alors que l'artisanat n'emploie que $13 \%$ du total des actifs de la Bavière, il forme $35 \%$ des apprentis (Handwerk in Bayern, 2007), au nombre de 88000 en 2006. Or les formations dispensées dans l'artisanat industriel ou tertiaire incluent l'impératif d'innovation. Cela se comprend aisément : les entreprises, grosses ou moyennes, travaillant dans les secteurs exposés à la concurrence mondiale, répercutent leurs propres exigences de compétitivité sur leurs partenaires artisanales.

Troisième caractéristique : la Bavière cumule les secteurs intensifs en savoir et se trouve spécialisée dans la production des biens d'investissement (36\% du CA de l'industrie en 2005 ) comme des biens intermédiaires (28\%), ce qui se reflète dans un taux d'exportation : $45,4 \%$ en 2005 - en hausse continue depuis 1998, où il n'était encore que de $37,3 \%$. Les secteurs-phares de l'économie bavaroise, identiques à ceux de l'Allemagne, enregistrent des taux nettement supérieurs : l'automobile $(61,5 \%)$, la construction mécanique $(57,5 \%)$ et l'électrotechnique $(55,0 \%)$. La répartition géographique de l'export du made in Bavaria reflète elle aussi dans l'ensemble la moyenne allemande : 60,2 \% des exportations sont allées dans l'UE, 13,2 \% dans la région USA/Canada et presque autant en Asie (2005).

Ces trois secteurs-phares font partie des trois ensembles-clefs qui réunissent près des deux tiers du total du chiffre d'affaires de l'industrie bavaroise (plus de 290 milliards $€$ en 2005). En tête figure la construction de véhicules, qui inclut l'automobile, l'aéronautique et le ferroviaire, avec $18,2 \%$ des emplois et $26,8 \%$ du CA (2005). On y trouve les constructeurs Audi (Ingolstadt), BMW ou MAN (tous deux dans la capitale), mais aussi nombre d'équipementiers comme Fichtel \& Sachs, Innowep GmbH (asservissement, Würzburg) ou Lechleiter $\mathrm{GmbH}$ (filtres, Munich). Dans le secteur du ferroviaire, si un groupe comme Siemens est spécialisé dans le matériel roulant (dont l'ICE ou le Transrapid), d'autres le sont dans les voies pour trains à grande vitesse, par exemple les sociétés RAIL.ONE GmbH ou Max Bögl Bauservice GmbH \& Co. KG, toutes deux implantées à Neumarkt dans le Haut Palatinat. Dans le domaine de l'aéronautique, la Bavière est le pôle leader en matière de R\&D outre-Rhin, aussi bien en ce qui concerne l'aviation civile (productions d'équipements pour Airbus) et militaire (Dornier, EADS, MTU), que le spatial : composants pour les lanceurs Ariane (MT Aerospace AG, par exemple), technologies satellitaires (Dasa, MBB, Astrium, IfEN ou Ketek) et de navigation (programme Galileo). Le cœur de ce segment se situe dans la région de Munich, où se trouve notamment le Centre de contrôle spatial Deutsches Raumfahrtkontrollzentrum (Oberpfaffenhofen). 
Diversité de l'industrie bavaroise et son apport à l'industrie allemande - quelques exemples (2005)

\begin{tabular}{|c|c|c|c|c|c|c|c|c|c|}
\hline & Entrep. & $\underset{(m r d \epsilon)}{\mathbf{C A}}$ & $\begin{array}{l}\text { Part dans } \\
\text { indus. B }\end{array}$ & $\begin{array}{l}\text { Part B. dans } \\
\text { indus. RFA }\end{array}$ & Salariés & $\begin{array}{l}\text { Part dans } \\
\text { indus. B }\end{array}$ & $\begin{array}{l}\text { Part B. dans } \\
\text { indus. RFA }\end{array}$ & $\begin{array}{c}\text { Taux } \\
\text { export }\end{array}$ & $\begin{array}{l}\text { Concentration régionale } \\
\text { (sites principaux) }\end{array}$ \\
\hline Construction auto. & 213 & 72,14 & $24,9 \%$ & $24,6 \%$ & 181685 & $15,8 \%$ & $22,8 \%$ & $61,5 \%$ & Munich, Ingolstadt \\
\hline Construction mécan. & 1000 & 40,15 & $13,8 \%$ & $22,4 \%$ & 191983 & $16,7 \%$ & $20,5 \%$ & $57,5 \%$ & $\Sigma$ du territoire \\
\hline Equipement électrique & 441 & 21,53 & $7,4 \%$ & $28,5 \%$ & 102436 & $8,9 \%$ & $25,3 \%$ & $48,7 \%$ & Munich, Nurembg., Ratisbon. \\
\hline Agro-alimentaire & 994 & 20,94 & $7,2 \%$ & $15,7 \%$ & 97188 & $8,4 \%$ & $18,6 \%$ & n.c. & $\Sigma$ du territoire \\
\hline TIC & 140 & 20,20 & $7,0 \%$ & $40,5 \%$ & 46880 & $4,1 \%$ & $30,6 \%$ & $67,5 \%$ & Région de Munich \\
\hline BTP & 14768 & 13,96 & $4,8 \%$ & $19,1 \%$ & 126108 & $11,0 \%$ & $18,1 \%$ & - & $\Sigma$ du territoire \\
\hline Chimie & 261 & 13,93 & $4,8 \%$ & $9,1 \%$ & 60248 & $5,2 \%$ & $13,7 \%$ & $49,4 \%$ & Burghausen-Trostberg-Gendorf \\
\hline Métallurgie & 846 & 10,87 & $3,7 \%$ & $12,9 \%$ & 75612 & $6,6 \%$ & $13,2 \%$ & $26,8 \%$ & $\Sigma$ du territoire \\
\hline Mesures/asservt., optique & 347 & 10,37 & $3,6 \%$ & $25,6 \%$ & 46000 & $4,0 \%$ & $19,4 \%$ & $65,4 \%$ & Haute Bav., Basse-Franconie \\
\hline Plastiques/caoutchouc & 532 & 10,26 & $3,5 \%$ & $17,5 \%$ & 67728 & $5,9 \%$ & $19,5 \%$ & $36,5 \%$ & $\Sigma$ du territoire \\
\hline Edition, imprimerie & 511 & 9,04 & $3,1 \%$ & $21,8 \%$ & 47597 & $4,1 \%$ & $19,9 \%$ & $13,8 \%$ & Mun., Nurem., Augsb., Wzbg. \\
\hline Bureautique, éq. informatiq. & 45 & 5,26 & $1,8 \%$ & $42,2 \%$ & 8141 & $0,7 \%$ & $30,1 \%$ & $12,5 \%$ & Haute Bav., Souabe (Augsbg.) \\
\hline Papeterie & 138 & 4,88 & $1,7 \%$ & $15,2 \%$ & 20889 & $1,8 \%$ & $15,2 \%$ & $53,0 \%$ a) & Hte Bav., Souabe, Bas. Franc. \\
\hline Aéronautique & 26 & 4,80 & $1,7 \%$ & $30,0 \%$ & 22579 & $2,0 \%$ & $30,4 \%$ & n.c. & Haute Bavière \\
\hline Prod./transfor. des métaux & 121 & 4,69 & $1,6 \%$ & $6,0 \%$ & 24153 & $2,1 \%$ & $9,7 \%$ & $36,6 \%$ & Moy. Franc., Souabe \\
\hline Mobilier & 208 & 4,59 & $1,6 \%$ & $21,6 \%$ & 27705 & $2,4 \%$ & $22,5 \%$ & $15,5 \%$ & Haute Franconie \\
\hline Mat. de construction minér. & 580 & 4,01 & $1,4 \%$ & $21,7 \%$ & 23294 & $2,0 \%$ & $22,7 \%$ & $19,1 \%$ & $\Sigma$ du territoire \\
\hline Textile & 180 & 2,91 & $1,0 \%$ & $22,5 \%$ & 17457 & $1,5 \%$ & $19,8 \%$ & $42,5 \%$ & Haute Franconie (Hof) \\
\hline Industrie du bois & 286 & 2,47 & $0,9 \%$ & $15,4 \%$ & 14507 & $1,3 \%$ & $17,4 \%$ & $21,0 \%$ & $\Sigma$ du territoire \\
\hline Habillement & 133 & 2,41 & $0,8 \%$ & $26,1 \%$ & 12284 & $1,1 \%$ & $29,1 \%$ & $36,9 \%$ & Basse et Hte. Franc., Hte Bav. \\
\hline Verre & 82 & 1,83 & $0,6 \%$ & $23,7 \%$ & 13171 & $1,1 \%$ & $25,5 \%$ & $42,2 \%$ & Haut Palatinat... \\
\hline Cuir & 45 & 1,50 & $0,5 \%$ & $40,1 \%$ & 6876 & $0,6 \%$ & $34,9 \%$ & $23,8 \%$ & Moyenne Franconie... \\
\hline Céramique & 58 & 0,99 & $0,3 \%$ & $32,2 \%$ & 11211 & $1,0 \%$ & $44,4 \%$ & n.c. & Haute Franconie \\
\hline Extraction de minéraux & 256 & 0,85 & $0,3 \%$ & $6,1 \%$ & 6061 & $0,5 \%$ & $7,0 \%$ & $14,2 \%$ & Hte Bav., Haut Palatinat \\
\hline Jouet & 34 & 0,66 & $0,2 \%$ & $38,1 \%$ & 4481 & $0,4 \%$ & $40,4 \%$ & $42,1 \%$ & Moyenne et Haute Franconie \\
\hline
\end{tabular}

Source des données : Bayerisches Staatsministerium für Wirtschaft, Infrastruktur, Verkehr und Technologie, en coopération avec l'institut Ifo, Industriebericht Bayern 2006, 10/2006. a) Production; transformation : $20 \%$ 


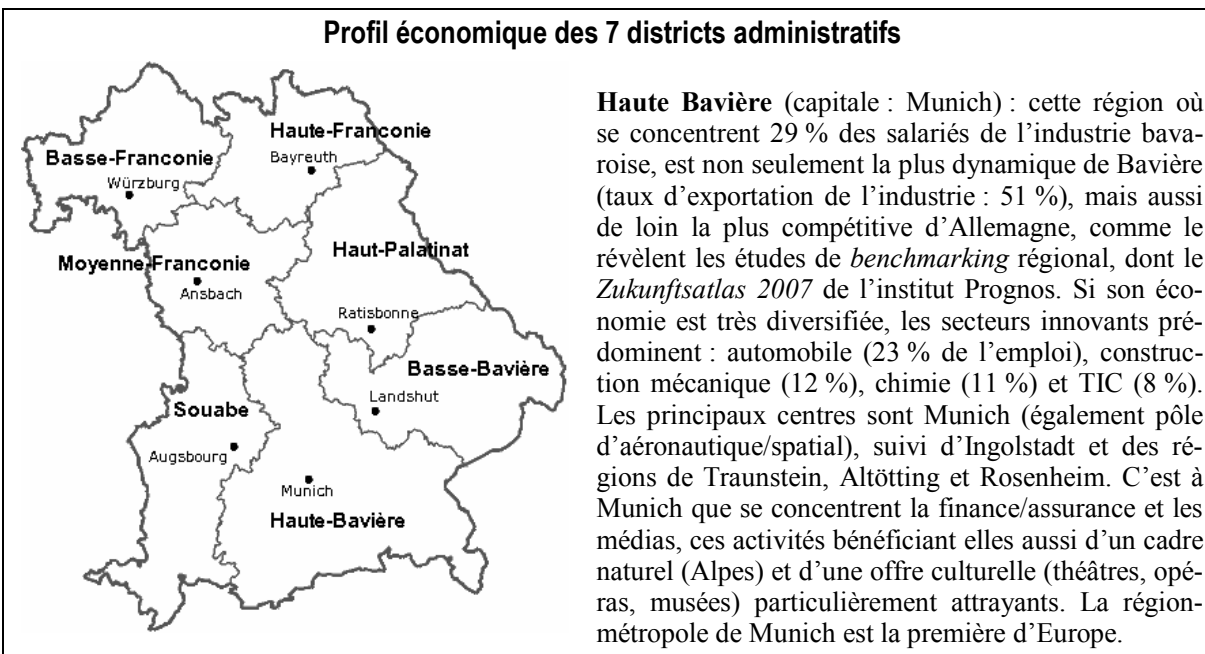

La Basse Bavière (capitale : Landshut) qui jouxte l'Autriche et la République tchèque est le fief des activités liées à l'automobile : sous-traitance et métallurgie (40\% de l'emploi industriel bavarois), qui se concentrent dans la région de Dingolfing-Landau. La construction mécanique vient en second lieu $(11 \%)$, suivie de l'agro-alimentaire $(9,5 \%)$. L'ensemble des secteurs industriels est représenté dans ce district (taux d'exportation: 44,5\%) qui souffre toujours de l'absence d'interconnexion entre les parties navigables est et ouest du Danube, le raccordement se heurtant au refus obstiné des écologistes.

Le Haut Palatinat (capitale : Ratisbonne) est la région moins industrialisée de Bavière (moins de $10 \%$ de l'emploi industriel du Land). Elle n'en est pas moins compétitive à l'international (taux d'exportation : $46,3 \%)$ du fait de ses spécialités : équipement électrique $(20 \%)$, automobile (14\%) ou construction mécanique $(13 \%)$ et métallurgie $(8 \%)$, de même que l'agro-alimentaire $(8 \%)$. Si les activités sont réparties sur l'ensemble du territoire, l'agglomération de Ratisbonne prédomine légèrement. La région présente un grand attrait touristique avec les monts du Fichtelgebirge, la forêt de Bohême ou le Danube.

La Haute Franconie (capitale : Bayreuth), qui jouxte la Thuringe et la Saxe, a longtemps souffert d'être acculée au Rideau de Fer. Moyennement industrialisée (10\% de l'emploi industriel du Land), ses activités sont encore faiblement tournées vers l'export (le taux est le plus faible de Bavière : 37,3\%), malgré le dynamisme de certains secteurs, comme l'industrie du caoutchouc et du plastique (14\% de l'emploi) ou la construction mécanique $(11 \%)$. L'équipement automobile en revanche $(10 \%)$ ou le textile $(8 \%)$ souffrent des restructurations générales de la branche. Ce district est par excellence le fief des PME, ce qui contribue à l'omniprésence de l'industrie sur son territoire. Les services n'en sont pas absents pour autant : assurance (la société HUK à Coburg), commerce (près de la moitié des emplois) ou tourisme naturel (Fichtelgebirge) et culturel (festival de Bayreuth, patrimoine : ville de Bamberg).

La Moyenne Franconie (capitale : Ansbach) est la seconde économie industrielle du Land (15\% des actifs; taux d'exportation : 42,2\%), avec une nette concentration dans l'axe Nuremberg-Fürth-Erlangen. Elle est vouée à l'électrotechnique $(19 \%)$, la construction mécanique $(17 \%)$ et aux technologies médicales $(6 \%)$, avec une prédominance de grosses PME et de grandes entreprises. La région, depuis toujours vouée aux échanges (foire de Nuremberg), accueille d'éminents prestataires de services comme KarstadtQuelle, les sièges de l'assureur Nürnberger Versicherungsgruppe ou de l'institut GfK. La région de Nuremberg partage avec Dresde le statut de « Gateway-Region » : pôle européen d'échanges est-ouest.

La Basse-Franconie (capitale : Würzburg) sur l'axe Rhin-Main est le fief de la construction mécanique (30\% de l'emploi ; taux d'exportation : 42,7\%), dominé par les PME. La région de Würzburg figure au $9^{\mathrm{e}}$ rang des dix sites high-tech européens répertoriés par Eurostat et au $4^{\mathrm{e}}$ dans le palmarès de l'institut Prognos. Points forts : mécatronique, TIC, technologies laser...

La Souabe (capitale: Augsburg), important pôle industriel (11\% du Land), est spécialisée dans les technologies de production et l'automatisation (construction mécanique : $24 \%$ de l'emploi) et l'agroalimentaire (12\%, région de l'Allgäu principalement). Si Augsburg est le $3^{\mathrm{e}}$ pôle d'activité bavarois après Munich et Nuremberg, et compte $70 \%$ d'emplois tertiaires, un grand nombre de régions reste voué à l'agriculture ou au tourisme (Lac de Constance, château de Neuschwanstein). 
En seconde position vient l'électrotechnique, qui comprend l'équipement électrique, les TIC, la bureautique et les mesures et asservissements ; ce secteur réalise 19,8\% du CA de l'industrie (17,7\% des emplois). Ce 'pôle' est à l'origine de l'industrialisation de la Bavière qui avait débuté par le transfert du siège de Siemens de Berlin à Munich au moment de la construction du Mur de Berlin (1961). Aujourd'hui, il comprend nombre de grands noms comme Osram AG ou Toshiba Europe. Le cœur de cet ensemble est constitué par l'équipement électrique, plus particulièrement les transformateurs et matériels de production/distribution d'électricité, un segment qui réunit à lui seul les trois quarts des salariés de l'électrotechnique. Si les TIC pèsent moins en comparaison dans l'économie bavaroise, la Bavière n'en est pas moins le site leader en Allemagne, avec 40,5\% du CA.

En troisième position par chiffre d'affaires vient la construction mécanique, avec $13,8 \%$ du CA ; mais elle est traditionnellement le plus gros employeur de Bavière, avec 16,7 \% de l'emploi industriel. Ce segment est hautement spécialisé dans les technologies de production et l'automatisation, intensives en savoir. Son point fort est la livraison de solutions et installations aux autres branches de l'industrie, comme l'agro-alimentaire, le textile ou le BTP (près de 50000 emplois), avec par exemple les sociétés Dörnhöfer Stahlbau Metallbau GmbH \& Co. (Kulmbach), Lindner GmbH (Freyung) ou Niedernhuber GmbH (Pocking). Il est suivi de la production d'éléments moteurs et de transmissions (FAG Kugelfischer ou SKF GmbH, toutes deux à Schweinfurt), puis de machines-outils (par exemple Maschinen Stumpf OhG, Rotthalmünster) et, en dernier lieu, d'électroménager (AEG/Electrolux à Nuremberg). Cet ensemble est par excellence le fief des PME : elles emploient près de la moitié des salariés.

Ces trois grands ensembles 'portent' littéralement l'industrie bavaroise, leurs activités étant liées étroitement à nombre d'autres secteurs : ils sont tributaires de la chimie (point fort : la chimie des spécialités, autre fief des PME), de la plasturgie ou encore de la production/transformation des métaux. Dans le même temps, ils tirent ces secteurs par leur demande, en attendant par ailleurs le même niveau de compétitivité et d'innovation que celui dont ils tirent eux-mêmes leur réputation mondiale. L'interdépendance sectorielle, les réseaux de partenariat entre grandes et petites entreprises ne se résument dès lors pas à des liens de sous-traitance, mais participent d'un effort individuel et collectif à la fois de performance globale, tel que peut le traduire par exemple un indicateur comme la part de la R\&D dans le PIB qui s'établit à $3 \%$ en Bavière, soit le taux projeté par les Etats membres de l'UE à l'horizon 2010 dans le cadre de la Stratégie de Lisbonne.

Cette culture de l'excellence, patiemment construite au fil des décennies en partenariat entre entreprises et pouvoirs publics, recueille aujourd'hui une reconnaissance mondiale, comme le rappelle fièrement le ministère Bavarois de l'Economie dans son Industriebericht 2006. La Bavière, qui figure aux côtés de 52 Etats parmi les 9 régions évaluées tous les ans par le Centre IMD de Lausanne qui édite le World Competitiveness Yearbook, s'est classée $16^{\mathrm{e}}$ en 2006 , alors que l'Allemagne ne figurait qu'au $26^{\mathrm{e}}$ rang.

\section{5-2005 : cinq étapes pour convertir une région déshéritée en site high-tech}

La Bavière a pu conquérir sa compétitivité actuelle grâce à une patiente politique de développement structurel régional favorisée par la continuité politique et par l'approche d'une action concertée entre pouvoirs publics et milieux économiques qui repose sur le principe de l'aide à 
l'auto-assistance. Dans cette acception, l'Etat comprend son rôle comme celui d'animateur, se contentant de fixer des objectifs généraux et d'entretenir un cadre favorable à l'activité. Cela inclut, dans la Bavière plus centralisée que les autres Länder, une politique généreuse d'aides à l'économie, et plus particulièrement de ce soutien au Mittelstand où cet Etat a fait figure de précurseur.

Concrètement, l'action engagée depuis la fin de la guerre se résume à trois grandes catégories : reconversion industrielle et technologique (de 1945 à aujourd'hui), création en parallèle d'un environnement favorable à la croissance (infrastructures, énergie, capital humain) et dorénavant, à l'aube de l'économie de la connaissance, développement durable de la compétitivité acquise par une politique active d'innovation.

Chronologiquement, la transformation de la Bavière a suivi 5 grandes étapes. La première, d'abord subie, a été l'afflux massif de réfugiés allemands d'Europe centrale : entre 1946 et 1950, le Land accueille 2 millions de réfugiés et d'Allemands des Sudètes, soit un cinquième de sa population totale. Or ces réfugiés, souvent hautement qualifiés, ont reconstruit leur existence en créant leur entreprise, qui dans l'agriculture, qui dans l'artisanat, qui dans la construction mécanique, suscitant par leur motivation un climat bénéfique à l'ensemble des activités. Et contribuant aussi, soit dit en passant, à tisser cette toile dense des PME qui est l'un des ressorts de la dynamique actuelle du site.

La seconde étape (années 1960) a été celle des localisations industrielles. Plusieurs facteurs ont été déterminants pour la genèse de l'industrie bavaroise. La partition de l'Allemagne incitait nombre de groupes industriels à transférer leur siège et leurs activités à l'ouest. Or la Bavière a attiré surtout des industries à orientation technologique prononcée : électrotechnique et électronique, automobile. Ainsi, le groupe Auto Union $\mathrm{GmbH}$, précurseur d'Audi, est fondé à Ingolstadt en 1947 (Hertrich/Mayrhofer, 2007); plus précisément : il s'agit d'un transfert à l'Ouest des activités de la firme originaire de Chemnitz (zone soviétique), fermée un an plus tard. Le groupe Siemens, originaire de Berlin, délocalise lui aussi ses activités, établissant à Munich et Erlangen les sièges respectifs de Siemens \& Halske et Siemens-Schuckert dès le blocus de Berlin. La Bavière y gagne en attractivité, d'autres industries suivent, et d'autres sociétés comme le groupe VIAG AG.

De VIAG à E.ON
VIAG était l'acronyme de Vereinigte Industrie-Anlagen-Aktiengesellschaft, une holding créée à Berlin en
1923 pour développer les industries de pointe de l'époque (aluminium et énergie en particulier). Dès les an-
nées 1930, VIAG construisait notamment des centrales hydrauliques en Bavière. Après 1945, VIAG avait
certes maintenu son siège à Berlin, mais installé l'administration du groupe à Bonn. Après sa privatisation en
deux étapes (1986 et 1988), VIAG transfère siège et administration à Munich en 1994, l'année où il prend le
contrôle de la principale compagnie d'électricité de la Bavière : Bayernwerk AG. En 2000, VIAG fusionne
avec VEBA, donnant naissance à l'énergéticien E.ON AG qui prendra le contrôle de Ruhrgas en 2003. Si le
siège de la holding E.ON AG est aujourd'hui à Düsseldorf (Rhénanie du Nord-Westphalie), la bavaroise de
ses deux filiales allemandes, E.ON Energie AG, est resté à Munich (l'autre étant E.ON Ruhrgas AG, Essen).

Ces implantations bénéficient d'une politique active d'incitation de la part du Land, renforcée en 1969 par l'adoption du programme de la Mission commune Bund/Länder « Amélioration de la structure économique régionale ». Celle-ci constitue toujours, parallèlement au fonds ERP héritier du plan Marshall, le dispositif par excellence de la politique régionale allemande. L'objectif des aides est double : veiller à l'équilibre territorial des zones d'activités, et donner aux entreprises les moyens de leur action. 
La troisième étape commence dans les années 1970, sur fond de choc pétrolier. Sous l'impulsion d'Anton Jaumann, ministre de l'Economie de Bavière de 1970 à 1988, le Land adopte le 8 octobre 1974 une Loi sur le soutien au Mittelstand (Mittelstandsförderungsgesetz), qui servira de modèle aux autres Länder, puis du Bund. L'optique du programme bavarois d'aide au développement des PME n'a pas varié depuis, il s'agit en priorité de susciter un comportement adapté à leurs besoins spécifiques chez tous les acteurs publics : administration de l'Etat bavarois, communes, fédérations, chambres de l'industrie, fondations et collectivités de droit public. Les moyens de cette politique incluent, certes, des aides publiques généreuses : subventions d'investissement versées par le gouvernement (il consacre quelque $10 \%$ de son budget à l'aide à l'économie), prêts spécifiques, ou crédits bonifiés délivrés par la filiale bavaroise de la LfA, banque publique aujourd'hui dédiée au Mittelstand. Ces aides ne visent pas à préserver des structures obsolètes ou à maintenir des acquis, au contraire : leur objectif est de créer des conditions favorables à l'épanouissement des entreprises. Sur la période 2000/04, par exemple, des prêts d'un montant de 1,2 milliard $€$ ont été accordés aux PME, se soldant par un volume d'investissement trois fois plus élevé et par la création de près de 23000 emplois (Mittelstandsbericht 2005). L'Etat de Bavière accorde toujours la même importance à cette politique, mais avec deux priorités nouvelles : l'aide à la création d'entreprises (du ressort du Land) et l'aménagement d'une fiscalité favorable à la succession (du ressort du Bund où, via ses diverses représentations, la Bavière mène un lobbying actif).

La quatrième étape s'enclenche dans les années 1980. Elle est dédiée à la recherche et aux technologies. La croissance économique du site étant bien engagée, il s'agit désormais de la stabiliser et, plus encore, de renforcer la compétitivité des entreprises à la fois dans les secteurs forts et dans les domaines clefs pour l'avenir. Ce sont donc essentiellement la construction mécanique et l'automobile, l'électronique et, dans une moindre mesure, l'industrie chimique qui bénéficient de ce nouvel axe de la politique bavaroise. En outre, la Bavière, soucieuse d'entretenir l'attractivité de son site naturel, soutient les technologies de l'environnement (dont le secteur mesures et asservissement), contribuant ainsi à hisser l'Allemagne en position de leader mondial. Plus globalement, la Bavière, déjà fortement internationalisée, cherche à valoriser ses atouts en les catalysant : une forte densité de PME innovantes car proches du marché et capables d'intégrer de nouvelles technologies dans des produits traditionnels ; un réseau tout aussi dense d'universités et de laboratoires de recherche, dont les Instituts Fraunhofer qui sont le relais recherche du Mittelstand, et qui favorisent les transferts de technologies vers les entreprises locales. La politique de recherche et technologie des années 1980 se concentre d'autant plus volontiers sur les PME que la Loi de 1974 sur le soutien au Mittelstand, modifiée en 1986 (et actuellement en cours de révision) poursuit expressément un objectif d'incitation à l'innovation.

Cinquième étape : dans les années 1990, la Bavière poursuit cette politique d'excellence avec des prolongements nouveaux, plaçant le high-tech au cœur de "l'Offensive Avenir ». Cette sorte de Stratégie de Lisbonne avant l'heure part du constat que l'Allemagne et la Bavière doivent d'urgence combler leur retard sur le Japon et les Etats-Unis. L'Etat bavarois déplace donc, mais sans rupture, les axes stratégiques de sa politique de développement des produits à technologie moyenne ou avancée vers les technologies de pointe, pour l'essentiel : TIC, technologies de l'environnement, biotechnologies, nouveaux matériaux et mécatronique. Mais ces secteurs particulièrement intensifs en R\&D exigent de la part de l'ensemble des pouvoirs publics un effort budgétaire supplémentaire. 
Or il ne peut être question de financer cette stratégie sur le budget du Land et des communes, leurs recettes fiscales ayant baissé de façon drastique au cours de la décennie. Le gouvernement bavarois refuse en outre tout accroissement de l'endettement car il enclenche dans le même temps sa consolidation budgétaire : lors de la présentation du 1999/2000 du Land à la fin janvier, le ministre des Finances Kurt Faltlhauser (il occupe toujours ce poste), annonçait que la Bavière se fixait pour objectif de renoncer progressivement à tout nouvel endettement d'ici 2009. Il s'agissait moins, pour la Bavière, de respecter les objectifs du Pacte de stabilité à la veille de l'adoption de la monnaie unique (bien que le bavarois Theo Waigel, ministre fédéral des Finances, démontre à la même époque son respect des critères de Maastricht), que de ne pas renier ses propres préceptes économiques. Le maître d'œuvre du « miracle économique », Ludwig Erhard, fut en effet le premier ministre de l'Economie de la Bavière avant d'être chancelier, se plait-on à rappeler en Bavière. La rigueur porte ses fruits : depuis 2006, le budget bavarois est équilibré, et la Bavière est la première collectivité territoriale à renoncer au déficit depuis 30 ans, se plait à rappeler son ministère des Finances. Cette politique lui permet d'avoir aujourd'hui (2007) le taux d'investissement le plus élevé d'Allemagne : 12,7 \% (à titre de comparaison : la moyenne des Länder ouest-allemands, hors villes-Etat, s'élève à 10,1\%). Et le plus faible endettement par habitant, avec $1849 €$ seulement à la fin 2006 (la moyenne des Länder se situe à 5 822€).

Pour financer son "Offensive Avenir », la Bavière avait lancé dès 1994 un ambitieux programme de privatisations en deux tranches, dont le fruit sert en priorité à amorcer l'investissement grâce au développement de la recherche, de la formation/qualification et, bien sûr, grâce aux créations d'entreprises situées au cœur des transferts de technologie. Ont ainsi été vendues notamment les parts que l'Etat détenait dans Bayernwerk AG, DASA ou Rhein-MainDonau AG. Au total, près de 3 milliards $€$ ont ainsi été dégagés sur la période 1995-2000 et attribués selon le principe de l'aide à l'auto-assistance. Le tiers de cette somme était destiné à la seule recherche scientifique (réacteur nucléaire scientifique de Garching, établissements supérieurs d'enseignement et de recherche, instituts Fraunhofer).

\section{Les fondations de l'excellence}

L'effort continu de reconversion repose sur une gestion 'de bon père de famille' visant à construire l'avenir sur des fondations qui ont fait leurs preuves. Encore fallait-il construire ces fondations. Au fil du temps, plusieurs chantiers ont ainsi été ouverts, soit en accompagnement de la politique industrielle, soit pour en construire le cadre. Ils s'agit de politiques conséquentes de développement structurel régional, de diversification énergétique, de désenclavement en matière d'infrastructures de transport et de valorisation permanente du capital humain.

Depuis la fin de la guerre, la Bavière bénéficie de plusieurs grands programmes de développement structurel régional. Jusqu'en 1993, elle était bénéficiaire des mécanismes horizontaux de solidarité du fédéralisme financier: au titre de ce Länderfinanzausgleich, elle a perçu au total 9 milliards $€$ (en équivalent de pouvoir d'achat 2006), avant de devenir ellemême contributrice (elle a versé depuis plus de 24 milliards $€$ ). La Bavière a également bénéficié des fonds de la Mission Commune Bund/Länder (Gemeinschaftsaufgabe) destinés à lisser les disparités territoriales.

Et elle bénéficie toujours des fonds structurels européens (développement des régions rurales, reconversion des sites industriels), quoique dans une moindre mesure aujourd'hui. 
Géographiquement, ceux-ci se concentrent principalement sur l'arrière-pays de l'ex-frontière interallemande (aujourd'hui limitrophe de la Thuringe et de la Saxe), ainsi que le long de la frontière tchèque ; le Rideau de Fer avait coupé ces régions de leurs zones d'échanges orientales sur $750 \mathrm{~km}$. A l'origine, ces aides étaient surtout destinées à y freiner l'exode économique consécutif à la fermeture des frontières du bloc de l'Est ou à y redynamiser le tissu économique restant. Aujourd'hui, il s'agit de soutenir les entreprises dans un contexte de concurrence nouvelle dû à l'ouverture des frontières en 1990, puis à l'élargissement de l'Europe. Certes, les activités ont connu un boom économique après cette normalisation, mais elles se sont trouvées confrontées à des difficultés d'un nouvel ordre, liées au retrait des forces militaires alliées, à la concurrence accrue de pays à bas salaires comme la République tchèque, et à la problématique spécifique aux branches prépondérantes dans ces régions (textile/habillement, porcelaine et verrerie). Les programmes de soutien ont donc été maintenus.

Au total, sur la période 1990-97, cette ex-zone frontière a bénéficié de quelque 2,5 milliards $€$ (fonds structurels européens, prêts et subventions versés par le Land, prêts au titre du programme de soutien au Mittelstand). Sur la période 2000-2006, les seuls fonds européens (essentiellement des fonds EFRE alloués au titre de l'Objectif 2) destinés à ces régions se sont élevés à 560 millions $€$; sur la période 2007-2013, 576 millions $€$ sont programmés, dont 84 millions $€$ pour la zone à la frontière tchèque. A cela s'ajoutent les divers mécanismes de soutien bavarois ou fédéraux, dont le programme bavarois "de mise à niveau pour l'UE» (EU-Ertüchtigungsprogramm) doté de 100 millions $€$ et qui se résume pour l'essentiel à des aides incitatives : jusqu'en avril 2004, 52 millions $€$ de subventions avaient été versés, générant des investissements d'un total de 340 millions $€$ (Bayern und die EUOsterweiterung, 2004).

Mais cette politique de soutien à l'émergence de nouvelles activités a aussi connu des dérives par le passé. L'exemple le plus patent en est le cas des aciéries Neue Maxhütte (Rosenberg) dans les années 1990. Pour la Bavière, il s'agissait de préserver des emplois dans le Haut-Palatinat, où ces aciéries étaient alors l'employeur presque exclusif ; condamner l'entreprise à la fermeture eût signifié le chômage pour quelque 10000 salariés. Face à cette urgence sociale, le gouvernement avait accepté de faire une entorse à sa doctrine, se laissant entraîner dans une spirale de subventionnement, finalement jugée contraire aux lois de la concurrence par la Cour européenne de Justice. Entre-temps, le Haut-Palatinat a opéré sa reconversion, et la Maxhütte a fermé en 2002.

Parallèlement à sa reconversion économique, le Land lance une politique énergétique offensive de diversification, les gisements bavarois de lignite, charbon, gaz naturel et pétrole étant largement insuffisants pour couvrir les besoins énergétiques décuplés par son industrialisation au cours des années 1960. De 1970 à 1996, la consommation d'énergie primaire a pratiquement doublé, passant de 1176 à 2021 PJ. Elle n'a plus guère varié depuis (en 2001, elle était de $2090 \mathrm{PJ}$ seulement), du fait notamment de la rationalisation de la production industrielle (elle consommait $20 \%$ de l'énergie bavaroise en 2003). En 2003, la consommation de l'industrie (8 688 tonnes équivalent charbon) était retombée en dessous de celle de l'année 1965 (9 021) après avoir atteint son point culminant en 1973 (11 613). En la matière, la Bavière ne déroge pas à la tendance globale de l'Allemagne. Pour assurer son approvisionnement énergétique, la Bavière a dû construire des infrastructures pétrolières (années 1960). Aujourd'hui, le Land, qui importe $100 \%$ de son pétrole, est raccordé au pipeline d'Europe centrale (de Gênes à Ingolstadt), et à la liaison transalpine (des pays de l'OPEC à 
Ingolstadt et au-delà via Trieste), qui acheminent le pétrole vers les quatre raffineries bavaroises, dont trois sont situées à Ingolstadt et alentours, et l'une à Neustadt sur le Danube. Ensuite, dans les années 1970/80, la Bavière développe son réseau de gazoducs, se raccordant notamment au réseau européen de distribution.

Production d'électricité par les compagnies publiques bavaroises 1955-2003 a) (principales sources en \% du total brut)

\begin{tabular}{|cccccc|}
\hline & Charbon $^{\text {b) }}$ & Fuel domestique & Gaz & Nucléaire & Hydraulique \\
1955 & 25,1 & - & - & - & 74,9 \\
1970 & 33,0 & 16,8 & 6,0 & 7,8 & 35,6 \\
1980 & 33,8 & 11,2 & 15,7 & 12,4 & 25,6 \\
1995 & 13,7 & 1,2 & 1,5 & 66,9 & 15,6 \\
2003 & 7,5 & 1,4 & 5,2 & 68,9 & 15,2 \\
\hline
\end{tabular}

Source des données : ministère bavarois de l'Economie, des Transports et des Technologies, Energiebilanz Bayern, 2006 (www.bayern.de). a) et des centrales de la DB Energie AG à partir de 2000 ; les compagnies publiques bavaroises produisaient 92,7 \% de l'électricité bavaroise en 2003 (14 $508 \mathrm{MW})$; b) houille, lignite et autres.

Après le choc pétrolier, la Bavière mise sur le nucléaire. Aujourd'hui, avec une part de près de $70 \%$ d'énergie d'origine nucléaire, la Bavière est le premier site producteur d'Allemagne (où la part du nucléaire dans l'Energiemix n'est que de $27 \%$ au total). Elle présente de grandes similitudes avec la France, avec laquelle elle entretient des relations de coopération étroites (projet EPR). Alors que, sous le premier gouvernement Schröder, l'Allemagne signait l'abandon du nucléaire, la Bavière y est toujours restée opposée. Et alors que, depuis l'arrivée au pouvoir de la Grande coalition et, surtout, la montée des risques pesant sur la sécurité de l'approvisionnement en gaz et pétrole importés comme la négociation sur la réduction des gaz à effet de serre, la question du nucléaire est remise à l'ordre du jour, la Bavière plaide activement pour le retour au nucléaire. Il en va de l'avenir de son approvisionnement en électricité, bien sûr, mais aussi et avant tout de la préservation de sa compétence technologique comme de celle de l'Allemagne en général.

Dès 1978, soit 20 ans avant l'arrivée au pouvoir à Berlin de la coalition SPD/Verts du chancelier Schröder, la Bavière lance un vaste programme de développement des énergies renouvelables. A la fin du siècle, la Bavière fournissait la moitié de l'approvisionnement allemand (total : 26 milliards de $\mathrm{kWh}$ ) en énergies renouvelables. Les centrales hydrauliques s'y taillent la part du lion avec deux tiers de la production totale; il est vrai que la Bavière est, à cet égard, privilégiée par sa topographie montagneuse. L'exploitation des autres ressources, pourtant largement subventionnée, n'est guère rentable encore. Si la Bavière reste tributaire de ses importations de matières énergétiques fossiles, elle est désormais largement autonome, voire exportatrice, en ce qui concerne l'hydraulique et, surtout, le nucléaire.

Le troisième axe est une politique des transports au long cours, qui atteint aujourd'hui son double objectif : le désenclavement et l'internationalisation du Land d'une part; d'autre part, l'aménagement régional du territoire par rééquilibrage des zones d'activité entre agglomérations et zones à faible activité (principe de l'équité des conditions de vie).

Coupée de ses voies de communication orientales par le Rideau de fer, la Bavière a dû développer ses infrastructures vers l'Ouest. Le canal Rhin-Main-Danube a ouvert un accès vital à l'axe industriel rhénan et à la Mer du Nord, tout en revitalisant les vallées du Main, du Danube et de ses affluents. Depuis l'ouverture des pays de l'Est, puis leur intégration dans l'UE, la Bavière retrouve sa place historique de zone d'échanges entre l'est et l'ouest de l'Europe. Mais il 
lui reste à convaincre les écologistes de la nécessité de rendre navigable une portion du Danube dans la région de Passau pour parachever la liaison mer du Nord-mer Noire. De même, le gouvernement Schröder ayant décidé de réduire considérablement sa participation budgétaire au développement des réseaux autoroutier et ferroviaire nationaux (Mission commune Bund/ Länder), l'extension des réseaux bavarois par-delà les frontières du Land, notamment vers les nouveaux Länder et les Etats riverains de l'est a été retardée. Or la Bavière ne saurait rester " plaque tournante du commerce et des transports " sans une "infrastructure performante " des transports, s'insurgeait alors le ministre bavarois de l'économie. Aujourd'hui, sous le gouvernement Merkel, les investissements dans les infrastructures sont redevenues une priorité politique, d'autant que par ailleurs l'extension des réseaux ouest- et est-européens, de même que leur interconnexion ne souffre plus aucun retard (Bourgeois, 2004).

Le second grand chantier de désenclavement a été le réaménagement/extension de l'aéroport de Munich, dont la première tranche s'est achevée en 1992. Il s'est traduit principalement par deux effets : d'abord un nouvel essor de l'internationalisation de l'économie bavaroise, grâce notamment à des liaisons directes avec les principaux pays partenaires de la Bavière, nécessaires également à l'expansion des principaux sites de foires-expositions bavarois (Munich, Nuremberg et Augsburg). Parallèlement en effet, les sites de Munich et Nuremberg se voyaient modernisés et considérablement agrandis. Ensuite, l'extension de l'aéroport FranzJosef-Strauss de Munich a eu un effet de dynamisation sur l'économie des agglomérations alentour, de Munich à Ratisbonne. Un deuxième terminal a été inauguré en 2003, et la construction d'une troisième piste d'atterrissage/décollage est à l'étude. Troisième chantier : après le réaménagement de la gare de marchandises de Munich-Riem et de la gare de triage de Munich-Nord pour rationaliser le ferroutage, la gare centrale de voyageurs de Munich a été replanifiée dans un concept intégré des transports ferroviaires, routiers et aériens (voyageurs et fret). Ces chantiers, financés par le Land, le Bund et les communes, ont permis à la Bavière de s'internationaliser et d'améliorer l'équilibre territorial des activités comme de l'emploi.

Plus décisive encore pour la dynamique du site est la valorisation du capital humain. Pour faire face au défi de la mondialisation, la première des conditions requises est de «disposer d'une main d'œuvre hautement qualifiée, tant en volume qu'en excellence, et qui soit à même en permanence de développer par elle-même ses compétences, de les mettre en réseau, d'avoir une vision à la fois globale et intégrée de son activité et d'agir en conséquence », écrivait ainsi en 1992 August R. Lang, alors ministre bavarois de l'économie (Programm Standort Bayern 2000).

Cet objectif nécessite une action à deux niveaux : d'une part, pour l'ensemble de la maind'œuvre, le maintien d'un haut niveau de qualification grâce à un système performant d'enseignement général comme de formation professionnelle initiale et continue. Si la formation générale bavaroise, qui continue de valoriser les disciplines fondamentales et les humanités, est unanimement considérée comme la meilleure d'Allemagne, le bât blesse en matière de formation professionnelle. Trop de jeunes bavarois en effet entament des études universitaires. Or cette 'sur-universitarisation' mène à une inadéquation avec le marché du travail et se révèle génératrice de chômage. Pour y remédier, la Bavière a mis en place, entre autres mesures, un ambitieux programme d'extension du dispositif des Fachhochschulen qui créent des passerelles entre formation professionnelle et enseignement supérieur et permettent d'acquérir une qualification professionnelle supérieure. Parallèlement, le gouvernement sensibilise les acteurs de la formation professionnelle (artisanat, chambres de commerce et 
d'industrie, entreprises) au développement de la formation continue, selon deux axes principalement : les nouvelles technologies et l'international.

D'autre part, la stratégie d'excellence exige un effort de formation d'élites dirigeantes. Or si, depuis le début du siècle, cette question est à l'ordre du jour des réformes allemandes de l'enseignement supérieur, la notion même de formation d'élites a longtemps constitué un profond tabou, que la Bavière s'est fait fort de briser. Ainsi, en avril 1999, une école internationale de management ouvrait ses portes à Munich : la Bayerische Elite-Akademie, accueillant une trentaine d'étudiants triés sur le volet, suivis chacun par un tuteur parallèlement à leurs études à l'université (sciences, économie, et sciences de l'ingénieur). L'originalité de cet établissement consiste dans sa complémentarité (les enseignements sont dispensés durant les vacances semestrielles) et dans son financement par les entreprises (dont BMW et Siemens). Cette création avait pour but de donner un signal fort pour résoudre un urgent problème qui affecte l'ensemble de l'économie allemande et plus encore celle de la Bavière : la relève des générations dans le Mittelstand. Nombre de ces PME familiales, créées après la guerre, sont aujourd'hui confrontées au problème des successions et cela, alors même que la concurrence internationale se renforce. Si de nombreuses petites entreprises sont ainsi menacées de fermeture faute de successeur, dans le même temps, une vague d'essaimage se dessine dans les grands groupes qui, en externalisant des pans entiers de leurs activités, créent de nouvelles entités, plus petites. Et entretiennent la demande d'excellence.

\section{L'axe stratégique du second millénaire : dynamiser l'excellence dans la société du savoir}

Le programme Allianz Bayern Innovativ, lancé en 2006, prolonge l'Offensive Avenir engagée en 1994 et son dernier volet (Offensive High-Tech) qui, sur la base des acquis en termes de compétitivité industrielle, mêlait trois axes: le développement des infrastructures et centres de compétences régionaux dans le domaine des technologies de pointe, leur mise en réseau et la poursuite de l'internationalisation du site. Elle était financée par le produit des privatisations (plus de 4 milliards $€$ au total). Déjà, la logique de cette politique était de rassembler les initiatives pour les optimiser et en faire naître des synergies nouvelles. L'approche de l'actuel programme est inscrite dans la continuité, mais avec une inflexion nouvelle : « aujourd'hui plus que jamais, la politique économique doit se nourrir d'une réflexion en termes de 'chaînes de création de valeur' "(Allianz Bayern Innovativ, 2006).

Cette politique repose dès lors sur deux piliers : une Cluster-Offensive qui vise à l'optimisation des 19 pôles de compétitivité sectoriels et high-tech existants grâce à leur mise en réseau virtuelle à l'échelle du Land et, parallèlement, le soutien au développement de pôles de compétences régionaux trans-sectoriels - version modernisée de la politique de cohésion régionale européenne habillée des couleurs du Land. La clef de cette approche : le programme Allianz Bayern Innovativ cherche à « impulser un processus ouvert d'auto-organisation ... reposant sur la mobilisation des forces propres des acteurs économiques ». Le Land se contente de mettre à leur disposition des outils plus que des moyens budgétaires: les clusters disposeront d'un financement initial de 50 millions $€$ au total sur cinq ans; les pôles régionaux trans-sectoriels bénéficient des mécanismes classiques : aides à l'économie du Land et/ou fonds EFRE.

L'Allianz Bayern Innovativ qui porte sur la période 2006-2011 a pour objectif d'accroître encore la compétitivité du site Bavière grâce à l'amélioration de la dynamique d'innovation, ${ }^{2}$ la hausse de la productivité et ce que le gouvernement bavarois appelle « le renforcement 
des attaches » liant les entreprises à leur territoire. Voilà, sous un habillage innovant, ni plus ni moins qu'une politique industrielle adaptée à une économie où la mobilité des facteurs joue un rôle déterminant. Le pivot en est le concept de réseau qui " garantit qu'une large partie de la chaîne de création de valeur restera en Bavière ». Il doit permettre de fidéliser les entreprises et secteurs les plus performants, ce qui permet en retour, une " communication offensive » sur les atouts du site aidant (voir www.invest-in-bavaria.de), d'attirer et de retenir les investisseurs étrangers.

\section{Le dispositif de l'ex-Offensive High-Tech au cœur de l'actuelle Cluster-Offensive}

L'Offensive High-Tech (1995-2005) était dédiée aux technologies-clefs : TIC, biotechnologies, génie génétique, nouveaux matériaux et mécatronique. Le programme se composait de deux volets, étroitement imbriqués : valorisation de la recherche et transfert de technologies, promotion d'une approche entrepreneuriale. Ce n'est au fond que le prolongement de la traditionnelle chaîne de l'innovation bavaroise : la Bavière tient en Allemagne le record des Prix Nobel scientifiques, et elle tient le record des dépôts de brevets. Universités, Instituts Max Planck, Instituts Fraunhofer, Offices allemand et européen des brevets, industrie, PME/PMI, y ont toujours fait bon ménage.

Les instruments de cette politique vouée à la promotion de l'esprit d'entreprise en général, étaient de trois ordres. Avant tout, il s'agissait de sensibiliser l'opinion et de dispenser informations et conseils aux créateurs potentiels (foires-expositions spécialisées, sites Internet). Parallèlement se créaient les infrastructures d'accompagnement: centres communaux de création d'entreprises, plateformes technologiques, interfaces de transfert de technologies et, bien sûr, infrastructures de communication à haut débit ont été ainsi implantés dans les régions au plus près à la fois des centres de production et des pôles de recherche. Enfin, s'ajoutaient à cela trois programmes transversaux d'innovation et de transfert de technologies, de capital-risque et de coaching, respectivement: BayIP (Bayerisches Innovationsförderungs-Programm, promotion de l'innovation process ou produit), BayTEP (Bayerisches Technologie-Einführungs-Programm; prêts aux PME désireuses de mettre en œuvre de nouvelles technologies dans leur production) et BayTOU (Bayerisches Programm zur Förderung technologieorientierter Unternehmensgründungen; accompagnement des sociétés à haut risque dans le domaine des technologies grâce à des subventions et du coaching). Si ce dernier programme se poursuit, les deux premiers sont aujourd'hui fusionnés au sein d'un programme de soutien aux technologies : Bayerisches Technologieförderungsprogramm (BayTP).

C'est ainsi que sont nés la plupart des clusters que vise à soutenir la nouvelle politique du Land. Par exemple le pôle de Munich-Martinsried, leader allemand incontesté dans le domaine des biotechnologies (génie génétique et thérapie génique). Les infrastructures de recherche de cette ancienne BioRegio constituent le premier élément de la mise en réseau des compétences, avec les instituts Max-Planck (biochimie, neurobiologie et psychiatrie), le CHU de Großhadern, le laboratoire de génie génétique de l'université de Munich et l'Institut d'hématologie du Centre de recherche GSF sur la santé et l'environnement (centre Helmholtz). Se greffe sur ce pôle de recherche la plateforme d'innovation et de création d'entreprise de Martinsried (IZB), qui a également pour rôle d'attirer des entreprises du secteur. Le pivot de ce réseau est la $\mathrm{Bio}^{\mathrm{M}} \mathrm{AG}$, une société créée $a d$ hoc à Munich en 1997 (financée majoritairement par les entreprises) pour gérer les subventions et assister toutes les parties prenantes, du montage du projet à la création de l'entreprise. Enfin, élément indispensable au fonctionnement de ce réseau : la communication professionnelle et grand public. Munich s'est donc dotée en 1998 d'un International Life Science Forum. Cette Bio ${ }^{\mathrm{M}} \mathrm{AG}$, qui œuvre sous le nom de $\mathrm{Bio}^{\mathrm{M}}$ Biotech Cluster Development $\mathrm{GmbH}$ depuis le 01-09-2006, est aujourd'hui la tête de pont du cluster virtuel de biotechnologies qui relie le dispositif de Martinsried aux pôles biotech de Ratisbonne et de Franconie (Bayreuth, Erlangen-Nuremberg, Würzburg). Il associe les deux universités de Munich (LMU et TUM), à celles de Würzburg, de Ratisbonne, d'Erlangen et de Bayreuth et au tissu des quelque 130 entreprises du secteur.

Au cœur de cette nouvelle politique de compétitivité globale se trouvent les 19 clusters retenus à l'issue d'une série d'études. Ils sont classés en trois catégories. La première est celle des clusters high-tech (biotechnologies, aéronautique/spatial, TIC, technologies environnementales et médicales); il s'agit de ces pôles d'innovation dynamisés par l'Offensive HighTech. La seconde catégorie rassemble des clusters centrés sur la production industrielle et les 
services liés : automobile, chimie, techniques sensorielles, énergie, agro-alimentaire, services financiers, logistique ou technologies ferroviaires - autrement dit, les points forts de l'économie bavaroise. La troisième catégorie, enfin, est celle des technologies transversales, le plus souvent encore en phase de recherche fondamentale : nanotechnologies, mécatronique, robotique et nouveaux matériaux.

Cette nouvelle politique, toute fidèle qu'elle soit à la Stratégie de Lisbonne révisée de la Commission qui cherche à marier aujourd'hui politiques d'innovation et de cohésion au niveau des régions, n'en a pas moins été longuement mûrie. Elle repose sur le bilan des politiques précédentes, sur un benchmarking permanent, mais aussi sur des études prospectives consacrées à l'évolution de l'économie mondiale et à ses grandes tendances. Si l'institut ifo de Munich évalue à intervalles réguliers divers axes de la politique économique du Land, l'institut Prognos (Bâle) s'était vu confier la réalisation d'un audit (Bayern 2020 - Industriereport) qui a largement inspiré les nouveaux choix du gouvernement bavarois. Il y rappelle que «la mission d'assurer la compétitivité relève en premier lieu des entreprises ». Le gouvernement ne peut agir qu'à deux niveaux : en veillant à ce que l'environnement des activités et, surtout, de l'investissement, soit porteur (mais les Länder partagent cette tâche avec le Bund) et en proposant des programmes de soutien ciblés à l'investissement. Cette approche est, depuis toujours, celle des gouvernements de la Bavière. Sur cette base, l'institut Prognos avait formulé cette préconisation : "Plus importantes que les programmes de soutien ciblés destinés à certaines branches ou entreprises nous semblent des mesures contribuant à l'intensification de la mise en réseau des entreprises bavaroises, des centres de recherche scientifiques et des partenaires allemands et étrangers $》$.

Les précédentes politiques d'innovation ont, semble-t-il, porté leurs fruits. La répartition géographique des divers pôles high-tech a rempli son objectif : valoriser les infrastructures existantes, garantir une répartition équitable des activités porteuses sur l'ensemble du territoire et promouvoir en les modernisant les bassins d'emploi. En même temps, comme les branches concernées constituent le cœur des activités du Mittelstand bavarois, principal créateur d'emplois, le marché du travail a été redynamisé par l'accroissement de la compétence et de la qualification de la main d'œuvre parallèlement à la création d'entreprises et d'emplois nouveaux grâce aux pépinières d'entreprises ou plateformes technologiques qui se trouvent au cœur du dispositif de transfert de technologies comme de R\&D.

Mais aussi performante soit-elle, la Bavière ne peut pas se reposer sur ses lauriers alors que s'effectue la transition de l'ère industrielle à l'économie du savoir et que la globalisation et, encore plus l'élargissement de l'UE, influent sur le partage mondial et européen du travail. "Même si la Bavière reste bien positionnée en comparaison allemande, cela ne suffit plus pour assurer le développement durable de son économie ", explique ainsi le ministère de l'Economie dans sa présentation de l'Allianz Bayern Innovativ.

VOILÀ LA NOUVELLE POLITIQUE de COHÉSION DE L'ETAT LIBRE DE BAVIÈRE : mettre en valeur les acquis pour mieux les optimiser en les adaptant aux grandes mutations. L'Alliance Bayern Innovativ peut se contenter aujourd'hui de donner une impulsion au changement en cours, tout en respectant la nature ouverte et dynamique de ce processus. Depuis toujours établie sur le principe de subsidiarité, la politique structurelle bavaroise avait permis la genèse d'une culture de l'innovation permanente. Si cette politique s'est caractérisée et se caractérise toujours par un volontarisme unique en Allemagne, elle n'en respecte pas moins 
l'initiative des acteurs économiques sans laquelle elle resterait lettre morte. La Bavière, bastion de l'orthodoxie du libéralisme organisé allemand, s'est mise à moderniser sa tradition de l'action concertée et du pilotage global. Si le rôle de l'Etat a toujours consisté à créer un climat favorable à l'effort collectif d'innovation, à la course à l'excellence de chacun des acteurs, la Bavière cherche aujourd'hui à mieux entretenir encore que par le passé ce cercle vertueux propice à la croissance et à l'emploi comme à l'innovation permanente : la mise en réseau des compétences, qu'elles soient humaines, sectorielles ou régionales, est l'approche unanimement considérée comme la mieux adaptée à l'économie de la connaissance.

Le 'miracle économique' bavarois n'a jamais correspondu à une quelconque 'vision'. Il est la résultante d'une dynamique cumulative que l'ensemble des acteurs a établie de concert, dans le contexte d'une étonnante continuité qui a permis au 'modèle' bavarois de s'adapter en permanence aux mutations. Or cette dynamique repose sur la conviction profonde qu'on ne peut construire l'avenir que sur des fondations durables. C'est cela que traduisait, sur un mode plutôt folklorique, le slogan de "l'alliance entre l'ordinateur portable et la culotte de peau » des années 1990. Sur un mode plus politique, la commission d'experts indépendants présidée par le Prof. Herbert Henzler (un ancien de McKinsey Europe) et instituée par le gouvernement bavarois pour préparer son futur programme d'investissements (Zukunft Bayern 2020), le formulait ainsi dans son rapport remis le 20 avril 2007 : "Le principe directeur des recommandations pour un programme d'avenir établi sur le long terme est celui du développement durable ». Ce principe va bien au-delà de la protection de l'environnement. Il implique avant tout une politique budgétaire saine, des investissements conséquents dans la formation et la recherche, condition sine qua non pour la croissance, un environnement favorable à la natalité et, plus que tout, une approche positive et constructive des mutations induites par la globalisation.

\section{Indications bibliographiques}

BAYERISCHE StAATSKANZLEI, High-Tech-Offensive Bayern: Bilanz und Perspektiven, janvier 2006 BAYERISCHES STAATSMINISTERIUM FÜR WIRTSCHAFT UND VERKEHR, « Programm Standort Bayern 2000 », Dokumentationen, $\mathrm{n}^{\circ} 3 / 92$

BAYERISCHES STAATSMINISTERIUM FÜR WIRTSCHAFT, VERKEHR UND TECHNOLOGIE, Zwei Jahrzehnte Bayerisches Mittelstandsförderungsgesetz (actes de colloques, volume 20, 1995)

BAYERISCHES STAATSMINISTERIUM FÜR WIRTSCHAFT, INFRASTRUKTUR, VERKEHR UND TECHNOLOGIE, Mittelstandsbericht 2005

BAYERISCHES STAATSMINISTERIUM DER FINANZEN, Bayerns Finanzen - ausgezeichnet!, avril 2007

Bayern 2020 - Industriereport. Analysen Trends, Prognosen, rapport réalisé par Prognos AG pour le compte du ministère bavarois de l'Economie, 2006

BlAnC C., Pour un écosystème de la croissance. Rapport au Premier ministre, Assemblée Nationale, Paris, 2004 Bourgeois I. et GuIOT C., "SIEMENS AG : de l'innovation à la performance globale », Regards sur l'économie allemande, $\mathrm{n}^{\circ} 70$, mars 2005

BouRgEOIS I., «Quelles infrastructures pour la Bohème au cœur de l'Europe ? », Regards sur l'économie allemande, $\mathrm{n}^{\circ} 66$, mai 2004

HERTRICH S. et MAYRHOFER U., "Audi AG: un succès signé made in Germany », Regards sur l'économie allemande, $\mathrm{n}^{\circ} 80$, mars 2007

HyPoVEREINSBANK, Fit für die Zukunft? Die Bundesländer im Vergleich, avril 2007

INSTITUT DER DEUTSCHEN WIRTSCHAFT, Viertes Bundesländerranking, septembre 2006

«L'économie de la Bavière », La Documentation française, Notes et études documentaires, 26-07-1971

www.invest-in-bavaria.de

Zukunft Bayern 2020, rapport du groupe d'experts présidé par le Prof. Herbert Henzler, avril 2007. 


\title{
Bade-Wurtemberg : une compétitivité à taille humaine
}

\author{
Isabelle Bourgeois
}

"Si le Land de Bade-Wurtemberg était un Etat et une économie autonomes, il serait champion du monde ". Voilà comment cette région se présente dans une plaquette destinée aux investisseurs, rappelant qu'en valeur des exportations par habitant (plus de $10000 €$ ) elle surclasse le Japon et les USA. Mais la fierté n'est pas dénuée d'humour. La présentation se poursuit ainsi : "Mais nous n'oserions jamais le proclamer haut et fort ». Un clin d'œil à l'image d'une tranquille et riche province de 'fourmis' économes, travailleuses et inventives qui marque ce Land industriel et high-tech qui rivalise de compétitivité avec la Bavière voisine. Et qui se plaît à rappeler que, s'il n'est que la troisième économie de la République fédérale en termes de PIB, il n'en surclasse pas moins l'Autriche, la Suède ou le Danemark au sein de l'UE. Surtout, le Land est la région la plus innovante d'Europe, loin devant l'lle de France et la Bavière en ce qui concerne la part du PIB consacrée à la R\&D (près de $4 \%$ ) ou le nombre de brevets par habitants (130 contre 115 pour la Bavière). *

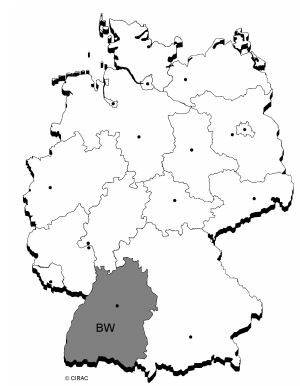

Réputé pour son attractivité touristique et gastronomique, le Bade-Wurtemberg $\left(35752 \mathrm{~km}^{2}\right.$, soit $5000 \mathrm{~km}^{2}$ de plus que la Belgique; capitale : Stuttgart), qui s'étend sur la rive droite du Rhin, de l'Alsace à la Suisse et à la Bavière, figure parmi les Länder les plus prospères d'Allemagne. Son PIB (294 milliards $€$ en 2005) représente $15 \%$ du total allemand et le place juste après la Rhénanie du Nord-Westphalie et la Bavière. C'est non seulement un poids lourd industriel, mais le site industriel allemand par excellence.

\section{Le site industriel allemand par excellence et premier exportateur}

A «l'alliance du laptop et de la culotte de peau » qui résume la politique d'innovation bavaroise, le Bade-Wurtemberg préfère une politique de compétitivité plus discrète, construite sur deux axes : la promotion de la culture industrieuse régionale grâce à un intense effort de formation professionnelle et de soutien aux activités de transfert de technologies et de savoirs, surtout dans les PME; l'aménagement d'un cadre favorable au développement des pôles de compétitivité qui ont émergé au fil du temps sur les piliers de l'industrie régionale (et allemande) : automobile, construction mécanique, TIC, biotechnologies et chimie. Mais l'internationalisation de ces branches qui faisait la force du Land risque aujourd'hui d'en fragiliser la compétitivité...

\footnotetext{
*Version actualisée d'un article paru dans Regards sur l'économie allemande, n 74, décembre 2005.
} 
Le Bade-Wurtemberg est le fief de ces biens d'investissement qui tirent traditionnellement la production manufacturière et les exportations de la République fédérale : la construction mécanique, l'automobile et l'électrotechnique. Il faut y ajouter une intense activité de R\&D : un quart des brevets déposés outre-Rhin est à mettre à l'actif de ce Land qui se présente volontiers comme " le pays des inventeurs et des philosophes » (das Land der Tüftler und Denker) et qui fut le berceau de l'automobile au XIXe siècle. On s'y plaît à rappeler que l'automobile a été inventée deux fois 'dans le pays' : par Gottlieb Daimler à Mannheim et par Karl Benz à Cannstadt. On y recense aujourd'hui des groupes comme DaimlerChrysler, Bosch, Mahle, Stihl, Kärcher, Heidelberger Druckmaschinen AG, Dürr AG, Carl Zeiss AG, Südzucker ou encore Roche, Michelin et Alstom, sans oublier Porsche.

La production manufacturière y contribue au PIB à hauteur de 38,4\% (2005; Arbeitskreis VGR der Länder). C'est beaucoup plus qu'en moyenne nationale, où la part de l'industrie est tombée à 29,3\%. C'est nettement plus aussi que dans cette Rhénanie du NordWestphalie qui fut longtemps le fief de l'industrie lourde mais qui, à l'issue d'une politique de restructuration originale, a aujourd'hui largement tertiarisé son économie : la part des services y dépasse les $70 \%$. Elle n'est que de 60,8 \% dans le Bade-Wurtemberg, ce qui, en termes relatifs, classe le Land au rang de l'économie la moins tertiarisée d'Allemagne.

Bade-Wurtemberg : industrie et structure des exportations en 2005

\begin{tabular}{|lccc|}
\hline Industrie & BW & RFA & Part dans RFA \\
Entreprises (nombre) & 8600 & 46600 & $18,7 \%$ \\
Salariés (en mio) & 1,202 & 5,928 & $20,3 \%$ \\
CA total (en mrd $€$ ) & 262 & 1489 & $17,6 \%$ \\
CA à l'export (en mrd $€$ ) & 123 & 604 & $20,4 \%$ \\
Taux d'exportation & $47,0 \%$ & $40,5 \%$ & - \\
\hline Exportations (en mrd $\epsilon$ ) & BW & RFA & Part dans RFA \\
Total & 123 & 786 & $15,6 \%$ \\
Automobile & 32 & 151 & $20,9 \%$ \\
Construction mécanique & 28 & 111 & $25,7 \%$ \\
Electrotechnique & 18 & 131 & $14,1 \%$ \\
Chimie & 12 & 103 & $12,1 \%$ \\
\hline
\end{tabular}

Source : Wirtschaftsministerium Baden-Württemberg, Jahreswirtschaftsbericht 2005/06

Mais ce qui semble un handicap recouvre en réalité le fait que les grands groupes industriels du Land ont intégré dans leurs structures leurs activités de services, et révèle également une très forte interdépendance 'intersectorielle' entre entreprises industrielles et prestataires de services, une structure dont le Land tire son avantage compétitif dans le jeu de la concurrence des sites allemands et européens. Car la moitié des activités tertiaires relève des services aux entreprises industrielles, ainsi que l'atteste la présence de sociétés comme SAP, ABB, Südleasing, Südfactoring ou Agilent Technologies Deutschland. Autrement dit : les deux tiers de l'activité du Land relèvent de cette industrie de services qui caractérise l'Allemagne ; la moitié seulement des activités tertiaires appartient au segment des services aux consommateurs.

Trois branches y réalisent les deux tiers du CA de la production manufacturière (plus de 261 milliards $€$ en 2005) et occupent plus de la moitié des actifs : la construction mécanique, 
l'automobile et l'électrotechnique. Elles exportent plus de la moitié de leur production. A elle seule, l'industrie automobile réalise plus d'un quart du total des exportations du Land (123 milliards $€$ en 2005), suivie de la construction mécanique et de l'électrotechnique. Les performances du Land sont stratégiques pour la croissance allemande, comme le révèle la part de ces trois branches dans le total des exportations de la RFA : respectivement 20,9\% (automobile), 25,7\% (construction mécanique) et 14,1\% (électrotechnique). Le BadeWurtemberg est le premier site exportateur de l'Allemagne : toutes branches confondues, le taux d'exportation de la production manufacturière bade-wurtembergeoise dépasse aujourd'hui les $47 \%$; il n'était encore que de 31,5\% en 1995.

Près des deux tiers $(58 \%)$ des ventes s'effectuent au sein de l'UE; c'est moins que la moyenne allemande $(63,4 \%)$. La France est le premier partenaire commercial européen du Land, avec quelque $10 \%$ de l'export (idem pour le total allemand), suivie de l'Italie (7,0\% ; moyenne allemande : $6,9 \%$ ) et de la Grande-Bretagne (6,7\% contre 7,8\%). Le gros tiers restant des exportations se répartit principalement entre les Etats-Unis (12\%), la Suisse $(5,7 \%)$ et la Chine $(2,9 \%)$. Hors de la zone Euro, le principal partenaire commercial du Bade-Wurtemberg sont donc les USA ; cette part nettement supérieure à la moyenne allemande $(8,8 \%)$ s'explique par la structure industrielle du Land où se concentre la production d'une part de ces biens d'investissement très demandés outre-Atlantique. Quant à la Chine, sa part est certes encore modeste mais connaît une très forte croissance.

Ces fortes activités à l'export sont bien sûr le fait de groupes industriels mondialement réputés et fortement internationalisés. 83 des 500 plus grands groupes allemands sont ainsi implantés dans le 'pays' de Mercedes, Lidl, MTU, Voith, Würth ou encore Hugo Boss. Mais ils ne sont que la partie la plus visible d'un très dense tissu d'entreprises dominé par des structures petites et moyennes et qui constitue de véritables grappes d'activités. L'exemple le plus classique en est le secteur automobile avec ses réseaux intégrant l'ensemble de la chaîne de création de richesse, de la conception au segment des équipementiers. D'une manière plus générale, les entreprises-phares des branches les plus ouvertes à la compétition mondiale 'animent' ainsi littéralement ces réseaux via contrats et commandes, et surtout en diffusant vers l'amont leurs impératifs d'innovation et de compétitivité. Elles contribuent de la sorte à ce que se développe un environnement porteur, bénéfique à la croissance et à l'emploi.

Mais ces global players sont loin d'être les seuls. Car le Bade-Wurtemberg, que ses habitants surnomment le Ländle (le « bon petit 'pays' »), est la patrie du small business ; il constitue le véritable socle des activités. Les PME représentent en effet l'écrasante majorité des entreprises, quelle que soit la branche. Une bonne moitié des établissements occupe au plus quatre salariés ; $2 \%$ seulement des entreprises en occupent plus de 50. La plupart des PME sont encore la propriété de la famille ou de la personnalité fondatrice. Nombre d'entre elles ont suivi une croissance continue au fil des générations. Certaines, désormais fortement internationalisées, figurent parmi les leaders mondiaux dans leur secteur. La liste de ces entreprises au CA s'échelonnant entre 165 millions $€$ et 1,65 milliards $€$ comprend l'équipementier automobile Eberspächer GmbH \& Co. KG (Esslingen), le spécialiste des tronçonneuses Stihl (Waiblingen), transformé en société par actions en 1997 seulement, le fabricant de machines-outils et spécialiste de la découpe au laser Trumpf GmbH \& Co. KG (Ditzingen), le spécialiste mondial de l'emballage aluminium ou plastique Hinterkopf GmbH (Eislingen), l'éditeur de jeux Ravensburger (Ravensburg, société par actions depuis 1988), ou encore le fabricant de trains électriques miniatures Märklin GmbH (Göppingen). 
Si ces PME sont mondialement réputées, la taille n'est pas le seul critère pour figurer parmi les hidden champions. On pourrait multiplier les exemples de ces petites et moyennes structures créées à l'origine autour d'un business modèle astucieux ou pour valoriser une invention. Elles sont nombreuses dans la région de Stuttgart, à forte densité industrielle, et dont les infrastructures de transport comme le haut niveau du capital humain sont particulièrement porteurs. On y trouve des références comme la société Leki Lenhart GmbH, spécialiste mondial du bâton de ski high-tech et issue en 1975 du plasturgiste Lenhart, le concepteur de lunettes de luxe Lunor AG, créé en 1991, ou encore le chocolatier Ritter-Sport $\mathrm{GmbH} \& \mathrm{Co} . \mathrm{KG}$, aux mains de la famille fondatrice depuis 1920. Mais la capitale est loin d'avoir le monopole de ces champions, répartis également sur l'ensemble du territoire.

\section{Un exemple de fonctionnement en partenariat : Hollister's MotorCycles}

Une société est à cet égard un exemple révélateur, bien qu'elle constitue un cas particulier puisqu'elle œuvre dans une niche au sein du segment du luxe. C'est la Hollister's MotorCycles, développeur et constructeur de motos réalisées sur mesure. La manufacture artisanale, sise à Zimmern-Horgen dans la ForêtNoire (elle ne communique aucun chiffre), ne produit qu'une vingtaine de motos par an, mais toutes faites main et destinées à l'export (USA, Monaco ou Arabie Saoudite). Elle est représentative à sa manière de ce mode de fonctionnement en réseaux de partenariats constitué autour d'un business modèle original, en l'occurrence dans la niche de l'automobile individualisée de luxe. Parmi les partenaires de Hollister's figure ainsi une autre manufacture, créée en 1988, spécialiste aujourd'hui mondialement réputée des roadsters sur mesure : la Wiesmann $\mathrm{GmbH} \& \mathrm{Co}$. KG, elle-même partenaire de BMW pour la motorisation. Le réseau de Hollister's inclut par ailleurs un prestataire de services électroniques (IBS Internet Business Services; www.black-forest.de) ou le studio d'un photographe d'art (Peter Hillert ; www.hillsalve.de) qui compte parmi ses clients Actea La Fenêtre confort ou Glaxo Smithcline Consumer Healthcare. S'y ajoutent l'hôtellerie de luxe (Interalpen Hotel Tyrol), de même que l'habillement sur mesure, avec les groupes belge Scabal et britannique Harper \& Fields.

La forte demande d'une offre individualisée dans le marché de masse par excellence qu'est l'automobile a incité l'équipementier Bosch à diversifier ses activités dans un créneau porteur, à la marge d'un segment du tuning en plein boom : celui des oldtimers. Au cours des dix dernières années, le nombre des véhicules de plus de 30 ans a en effet augmenté de $250 \%$ sur le seul marché allemand; leurs propriétaires dépensent en moyenne quelque $1000 €$ pour leur entretien. Le groupe a créé en 2005 une nouvelle division opérationnelle : Bosch Automotive Tradition. Quatre pays sont visés, les fiefs de l'automobile de collection : Allemagne, France, Grande-Bretagne et Italie.

On voit là comment, de proche en proche, se créent des synergies transsectorielles qui intègrent d'entrée de jeu l'espace européen ou mondial et dynamisent un territoire. Les entreprises sont insérées dans un environnement régional hautement compétitif, fondé sur une culture de la concurrence et de l'excellence qu'elles partagent toutes, quelle que soit leur taille. Cette culture n'est pas propre au seul secteur automobile, contrairement à ce pourrait laisser croire l'existence de champions dans le segment de l'équipement comme Behr GmbH \& Co. KG (Stuttgart), leader de la climatisation et du chauffage automobile. Ces champions se recrutent dans l'ensemble des activités, comme le montre l'exemple de la société $\mathrm{M}+\mathrm{W}$ Zander Holding AG (Stuttgart) qui, en l'espace de 10 ans, a décuplé son CA et s'est hissée au rang des leaders dans le segment du facility management à partir de son cœur de métier : le microprocesseur.

La compétitivité de ces entreprises est liée à plusieurs facteurs. Le premier est la forte réactivité que permet leur taille petite ou moyenne, conjuguée à des circuits décisionnels 
courts entre l'identification de la demande et la proposition de solutions au client. A cette forte proximité avec le marché s'ajoute un second facteur: la densité des activités industrielles et de services liés sur l'ensemble du territoire du Land. Autrement dit : les fournisseurs des entreprises citées et de tant d'autres sont eux aussi hautement réactifs et performants. C'est là le reflet du classique fonctionnement en réseau des PME allemandes. Enfin, troisième facteur : l'intensité de leurs activités de $\mathrm{R} \& \mathrm{D}$, particulièrement prononcée dans le Bade-Wurtemberg qui consacre quelque $4 \%$ de son PIB à la $R \& D$. Mais il ne s'agit là que d'une moyenne. La société Trumpf, par exemple, consacre $8 \%$ de son CA à la R\&D - deux fois plus que la moyenne de sa branche. Elle s'est vu décerner en octobre 2005 le prix du Best of European Business (catégorie « Innovation »; entreprises au CA inférieur à 3 milliards $€$ ), créé dans sept pays européens (dont la France et la Grande-Bretagne) par le cabinet Roland Berger, le quotidien Financial Times et le périodique manager magazin. L'autre lauréat dans la catégorie innovation (CA supérieur à 3 milliards $€$ ) était le constructeur automobile bavarois BMW. Deux autres groupes du Bade-Wurtemberg se sont vu décerner le prix la même année, dans la catégorie «croissance exceptionnelle»: le constructeur automobile Porsche et le groupe pharmaceutique Altana AG (Constance, CA : 3 milliards $€$ ).

\section{Homogénéité territoriale et partage interrégional du travail}

Le quatrième facteur est une caractéristique unique outre-Rhin, qui distingue le BadeWurtemberg des autres Länder, même de la Bavière voisine : une grande homogénéité territoriale en termes d'activités, de productivité ou d'innovation, c'est-à-dire l'absence de disparités régionales prononcées. Les activités sont certes concentrées dans les zones urbaines; Stuttgart, Böblingen et Heidelberg constituent le trio de tête des agglomérations les plus compétitives. Mais les banlieues ou les zones à faible densité de population ne sont pas moins dynamiques. Près de la moitié des régions du Land sont hautement compétitives, ce qui porte la part du Bade-Wurtemberg à $20 \%$ de l'Allemagne, estime l'institut Prognos. L'ensemble du Land constitue dès lors comme un pôle de compétitivité d'un seul tenant.

Bien sûr, la structure des activités varie au sein du Land, délimitant son territoire en quatre principaux bassins qui suivent grosso modo les frontières des quatre districts administratifs (et des territoires des douze chambres de commerce et d'industrie). Or ces différences donnent lieu à une sorte de "partage interrégional du travail » (Arndt et al., 2005), à l'origine de la compétitivité du Bade-Wurtemberg, puisqu'il associe zones dédiées au savoir et aux services, donc à capital humain hautement compétitif, et zones dédiées à l'industrie et la $\mathrm{R} \& \mathrm{D}$, à forte intensité en capital.

En matière d'industrie et de $R \& D$, une région domine de loin : celle de Stuttgart. La capitale héberge l'une des six bourses régionales allemandes et le siège de 22 grands groupes, ce qui la classe au $5^{\mathrm{e}}$ rang après Hambourg, Munich, Düsseldorf et Essen, comme le rappelle une étude commanditée à Prognos par la banque publique du Land (L-Bank). Quatre branches y dominent: automobile, électrotechnique et informatique, édition et médias, assurance. Cette structure fait de Stuttgart la métropole la plus industrielle d'Europe : $40 \%$ de la création de valeur y est imputable à l'industrie (Werner et al., 2005) ; c'est 10 points de plus que les agglomérations de Milan et Turin, ou que la moyenne européenne. Elle lui confère aussi la $2^{\mathrm{e}}$ place parmi les métropoles les plus créatrices de richesse d'Allemagne, après Munich. Au sein de l'UE, elle occupe le $9^{\mathrm{e}}$ rang (Munich, Londres et 
Copenhague constituant le trio de tête). Car à la différence des autres métropoles où dominent de loin les services, la structure majoritairement industrielle de Stuttgart a pour corollaire un énorme potentiel d'innovation. L'Office des statistiques du Land y a dénombré 876 brevets déposés par million d'habitants en 2002 ; c'est moins que Munich, mais la région de Stuttgart investit 5,78 \% de son PIB dans la R\&D - un record outre-Rhin, souligne la CCI.

4 districts administratifs.

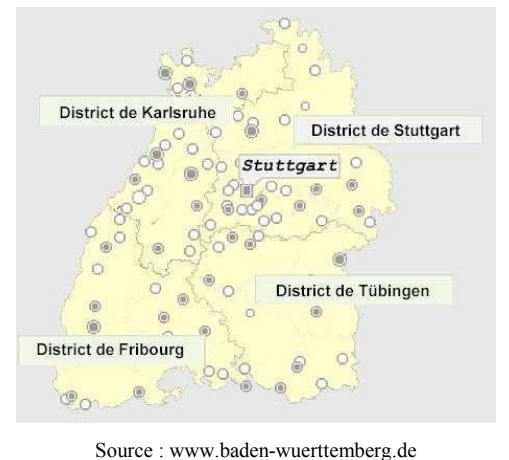

Le Bade-Wurtemberg est le seul Land à avoir été créé par referendum. Il est issu en 1952 de la fusion de trois Länder créés après la guerre dans les zones d'occupation américaine (Nord-Württemberg-Baden) et française (Süd-Baden et SüdWurttemberg-Hohenzollern). Les districts administratifs actuels en suivent les anciennes frontières. Depuis 1952, le parti CDU est majoritaire dans le Land, composant le gouvernement tantôt seul, tantôt au sein d'une grande coalition avec le SPD (1966-72 et 1992-96), ou en s'alliant aux libéraux, ce qui est le cas depuis 2005. Ministreprésident : Günther H. Oettinger (CDU).
... et un réseau de $12 \mathrm{CCl}$

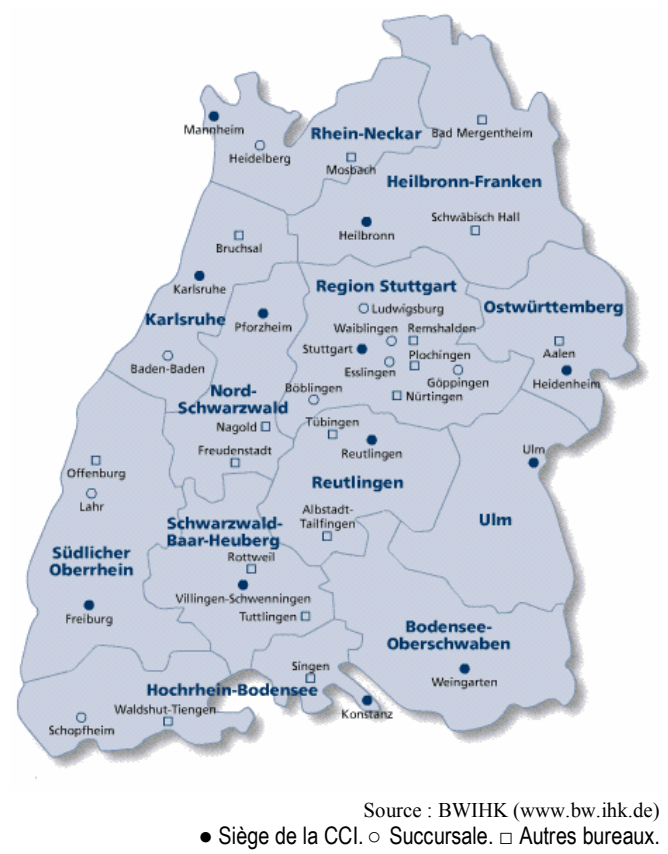

Cela s'explique par la forte présence de l'automobile et de la construction mécanique, les traditionnels moteurs de l'innovation incrémentale outre-Rhin. A titre d'illustration: le «Prix Avenir » allemand 2005 (Deutscher Zukunftspreis) avait été décerné par le président de la République à une équipe pilotée par deux ingénieurs, employés respectivement par Siemens (Bavière) et Bosch (Stuttgart) ; ils avaient développé le système d'injection piézoélectrique pour les moteurs diesel et essence. La présence de ces branches-phares entretient un environnement favorable à la création de start ups comme cette Foldcore $\mathrm{GmbH}$, créée en 2004 (www.foldcore.de) par deux ingénieurs sur la base d'un procédé du même nom développé par eux. Le procédé de pliage 'en sandwich' de matériaux fins, mais souples, comme le carton ou les tôles, permet de produire à faibles coûts des matériaux légers, drainants, acoustiquement isolants, destinés au BTP, à l'automobile ou à l'aéronautique. Un de ses premiers clients est d'ailleurs Airbus. L'intense activité industrielle dope aussi les services aux entreprises, comme le révèle le parcours de la Bernd Kußmaul GmbH (Weinstadt/ Großheppach), créée en 1997 par un technicien de la construction mécanique et diplômé en sciences de gestion (www.bernd-kussmaul-gmbh.de). Sa société, qui occupe 15 salariés, s'est spécia- 
lisée dans le management de projet et le supply chain management; parmi ses premiers clients figuraient Audi Quattro GmbH et Adidas.

\section{Benchmarking régional des pôles de compétitivité allemands en termes de potentiel}

Région de type A Région de type $\mathrm{B}$ Région de type C Région de type $\mathrm{D}$ Région de type $\mathrm{E}$ Région de type $\mathrm{F}$ Région de type $\mathrm{G}$

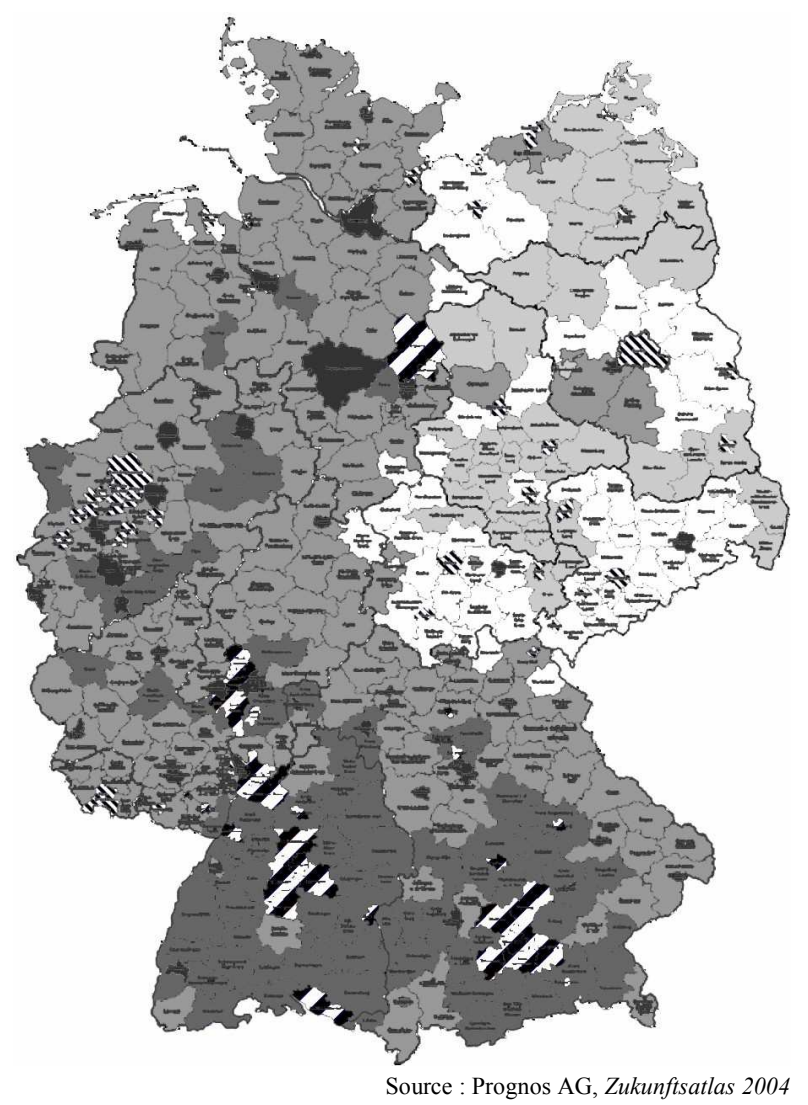

La carte ci-dessus détaille le potentiel - non pas l'état actuel - de compétitivité des 439 circonscriptions administratives et agglomérations recensées en Allemagne, tel qu'il a été établi par Prognos. L'Institut a déterminé à cet effet sept types de profils ; ils se composent de 4 catégories d'indicateurs : démographie, marché de l'emploi, concurrence et innovation, prospérité et situation sociale.

\begin{tabular}{|c|c|c|c|c|}
\hline Type & Démographie & Emploi & Concurrence/Innovation & Prospérité \\
\hline A & ++ & ++ & ++ & ++ \\
\hline B & ++ & ++ & + & -- \\
\hline C & ++ & $\mathbf{0}$ & $\mathbf{0}$ & ++ \\
\hline D & + & $\mathbf{0}$ & - & + \\
\hline E & - & $\mathbf{0}$ & - & -- \\
\hline F & - & - & -- & $\mathbf{0}$ \\
\hline G & -- & -- & -- \\
\hline
\end{tabular}

La carte révèle que la quasi-totalité du Bade-Wurtemberg appartient au moins au type $\mathrm{C}$. Ce classement s'explique par la forte compétition régionale au sein de la RFA. Si le Bade-Wurtemberg est hautement compétitif aujourd'hui, d'autres Länder se caractérisent par un dynamisme prononcé qui pourrait à l'avenir modifier sa position. Dans cette approche de benchmarking, seule l'agglomération de Stuttgart et ses environs, de même que le district du Lac de Constance ou la région Rhin-Neckar figurent parmi les clusters allemands (type A) les mieux armés pour l'avenir. 
Au sein de la région de Stuttgart, un site s'est spécialisé dans le développement de logiciels et de hardware : Böblingen/Sindelfingen au sud-est, où sont installées des sociétés comme Hewlett Packard, Agilent Technologies, Philips Semiconductors, le second centre de recherche mondial d'IBM ou la Smart GmbH (DaimlerChrysler). Elle constitue un cluster certes particulier, mais intégré dans la structure des activités de la région. Ces groupes se sont associés avec les pouvoirs municipaux et régionaux dans une «Initiative de développement économique » au sein de laquelle ils mettent en réseau leur savoir-faire afin de le rendre accessible aux autres entreprises du cru, notamment aux PME. Dans ce cadre, le gouvernement du Land, les municipalités et la CCI locale avaient lancé en 1995, avec le soutien de DaimlerChrysler, HP et IBM, un « Centre du logiciel » rassemblant dans un réseau virtuel de coopération (www.softwarezentrum.de) 80 PME du secteur, anciennes et nouvelles.

Si la région de Stuttgart est le leader incontesté parmi les zones dédiées à l'industrie et la $\mathrm{R} \& \mathrm{D}$, elle n'est pourtant pas la seule. A l'est, l'agglomération d'Ulm et, au sud, la région Bodensee-Oberschwaben font partie elles aussi de ces pôles industriels à fort dynamisme. Cette dernière région, qui partage le lac de Constance avec la Suisse et l'Autriche, héberge de grands groupes tels ZF Friedrichshafen, MTU, EADS Astrium ou EADS Dornier, mais aussi des entités moyennes comme VA Tech Escher Wiss GmbH, Hawera Probst GmbH (outillage), sans oublier Ravensburger. La région, pourtant de structure à dominante agri- et sylvicole, compte parmi les plus dynamiques en termes d'innovation, avec 106 brevets pour 100000 habitants selon une étude de Prognos effectuée en 2002 (et citée par la CCI), ce qui la situe aujourd'hui au quatrième rang des régions d'Allemagne. Elle doit sa compétitivité à un recentrage collectif sur son cœur de compétences : l'automobile et la construction mécanique - les deux branches les plus en pointe à l'international.

L'agglomération d'Ulm, stratégiquement bien située sur l'axe Munich-Stuttgart, se caractérise quant à elle par le développement d'un cluster mettant en synergie la R\&D d'entreprise avec la recherche fondamentale et appliquée de l'université et des établissements extrauniversitaires dans des domaines à première vue disparates, mais dont la coexistence reflète en réalité la diversité des enjeux liés aux technologies-clefs : médecine, chimie-biologie, physique, informatique ou mathématiques, sans oublier les technologies liées à l'énergie ni les sciences économiques et de gestion. Par ailleurs, tous les niveaux de qualification sont ainsi rassemblés - du technicien formé dans la Fachhochschule à l'ingénieur diplômé de l'Université d'Ulm -, ce qui contribue à une large diffusion de la culture de l'innovation, favorable à la réactivité des entreprises comme à la promotion de la recherche fondamentale. On y observe une forte concentration de centres réputés comme l'Institut für Lasertechnologien in der Medizin und Meßtechnik (ILM, technologies du laser appliquées à la médecine et aux mesures et asservissement), I'Institut für Diabetestechnologie (technologies du diabète) ou le Zentrum für Sonnenenergie- und Wasserstoffforschung (ZSW, énergie solaire et recherche sur l'hydrogène). S'y ajoute le centre de recherche de DaimlerChrysler, spécialisé notamment dans les matériaux, la microélectronique ou l'énergie. Ces compétences sont réparties dans l'ensemble de l'agglomération, mais mises en réseau grâce à une politique de coopération lancée en 1986 et associant entreprises, pouvoirs locaux et dispositif de recherche dans une approche partagée de transfert des savoirs et compétences.

Cette stratégie qui fait d'Ulm une «Cité des sciences» (Wissenschaftsstadt) avait pour objectif délibéré de permettre la reconversion d'une ancienne région industrielle vers un site compétitif dans l'économie du savoir. Dans le cadre de sa politique d'urbanisme et de déve- 
loppement économique, la municipalité a piloté et soutenu la création de trois Science Parks. "On ne peut pas forcer le succès; mais on peut optimiser les conditions-cadre », explique la ville (www.ulm.de). L'étude 2004 de Prognos sur la compétitivité des sites valide a posteriori cette approche. Elle se fonde aussi sur une coopération étroite avec l'agglomération voisine de Neu-Ulm, située sur la rive bavaroise du Danube, en matière de politique structurelle virtuelle (e-gouvernement). Cette politique interrégionale a reçu en novembre 2005 le Prix «Best-Practice-Award MEDIA@Komm-Transfer » décerné conjointement par le ministère fédéral de l'Economie et diverses entreprises dont SAP et T-Systems.

Dans la catégorie des régions dédiées aux services et aux savoirs, la ville de Heidelberg se profile comme leader en matière de biotechnologies, de TIC et de services financiers. Elle est au cœur de cette BioRegion Rhein-Neckar-Dreieck (triangle Mannheim, Ludwigshafen, Heidelberg au confluent du Neckar et du Rhin) qui est l'un des trois sites dédiés aux sciences du vivant lauréates du concours BioRegio lancé par le ministre fédéral de la Recherche en 1996 et destiné à rapprocher la recherche fondamentale du marché. Dans ce cluster se concentrent en effet d'une part des structures réputées de recherche en biologie moléculaire comme le laboratoire européen EMBL créé en 1974, le centre de recherche ZMBH de l'Université Ruprecht-Karls, l'Institut Max-Planck de recherche médicale ou le centre de recherche allemand sur le cancer DKFZ, une fondation du Land de Bade-Wurtemberg qui est membre de la société Helmholtz et de la DFG. Ces structures, spécialisées notamment dans la biogénétique ou la bioinformatique, et qui sont toutes à Heidelberg, travaillent en réseau avec les pôles de technologie moléculaire, d'informatique et de mathématiques appliquées de Mannheim, ceux de microbiologie de Kaiserslautern ou ceux de biogénétique de Neustadt/Weinstraße (biotechnologies vertes). S'y ajoutent d'autre part des géants de la chimie/ pharmacie installés dans les sites alentour (Roche Diagnostics, Merck, Abbott, Baxter, ou Aventis Pasteur) et un certain nombre d'entreprises innovantes à fort potentiel de croissance (Lion Biosciences, Leica Microsystems GmbH...). La plateforme technologique de Heidelberg (Life-Science-Park), sise sur le campus de l'université, est la première d'Allemagne, avec 45 entreprises et quelque 1000 salariés.

La ville de Heidelberg ( $80 \%$ des activités dans le tertiaire) est aussi un de ces pôles où se concentrent les services aux entreprises (l'éditeur de progiciels SAP, le courtier en assurances MLP AG ou les éditions scientifiques Springer). Or cette tertiarisation ne se fait pas au détriment de l'industrie, qui au contraire s'insère parfaitement dans ce schéma d'innovation, comme le révèlent les noms des entreprises qui ont leur siège dans la ville : Heidelberg Druckmaschinen AG (presses d'imprimerie, leader mondial), Heidelberger Zement AG (cimenterie), ABB AG, spécialiste de la robotique et des technologies de l'énergie, ou encore le fabricant de stylos Lamy GmbH, une PME familiale au CA de 50 millions $€$. En termes de potentiel de compétitivité, la ville de Heidelberg, pôle universitaire d'excellence, surclasse Stuttgart; elle bénéficie d'une structure démographique particulièrement dynamique, avec une forte proportion d'actifs jeunes et hautement qualifiés.

Deux autres pôles urbains tertiaires se distinguent: Fribourg-en-Brisgau et Karlsruhe. La structure économique de ces villes universitaires et touristiques est dominée par la banque et l'assurance, le logiciel (autrement dit : des services aux entreprises), ainsi que les technologies liées à l'environnement. Fribourg est ainsi le premier pôle de compétence allemand dans le domaine de l'énergie solaire, ce qu'expliquent la présence de l'Institut Fraunhofer spécialisé en la matière (ISE), d'entreprises comme Solarfabrik AG et Solarstrom AG ou 
d'institutions comme l'International Solar Energy Society (ISES), sans oublier les activités du principal salon européen du secteur, l'Intersolar. Parallèlement, la situation géographique de Fribourg (ouverture sur trois marchés nationaux) comme son dispositif de recherche universitaire et appliquée ont amené cette autre BioRegio allemande à s'associer avec les villes limitrophes (Strasbourg, Bâle et Karslruhe) dans un réseau de coopération transfrontalière : la Biovalley du Rhin supérieur, pôle biotechnologique d'envergure européenne.

La Technologiefabrik de Karlsruhe (www.technologiefabrik-ka.de) est l'une des premières pépinières et plateformes technologiques créées en Allemagne, à l'initiative du Land qui lançait là, dès 1983, les axes d'une politique de développement structurel et de compétitivité globale cherchant avant tout à favoriser la mise en réseau des compétences. Avec deux objectifs : encourager une approche proactive de l'innovation dans les PME, et promouvoir la création de start ups technologiques grâce à une politique d'offre, ce qui distingue la politique du Bade-Wurtemberg de celle menée dans l'Alsace voisine, où la priorité est la sensibilisation à l'innovation (De La Paix, 2001). Rappelons que l'existence d'une forte culture d'innovation est l'une des caractéristiques foncières du Bade-Wurtemberg - et ce, depuis le milieu du XIX siècle. La « Fabrique technologique » de Karlsruhe, installée dans l'ancienne manufacture de machines à coudre Singer à quelques minutes de l'université, est ainsi reliée à un plus vaste réseau régional d'incitation à la création d'entreprise, à la recherche fondamentale et appliquée, et au soutien au Mittelstand.

Et elle est membre de la fédération de ces plateformes (ADT) qui compte plus de 160 adhérents, dont 17 dans le Bade-Wurtemberg. Au fil du temps, quelque 240 entreprises sont nées dans l'incubateur de Karlsruhe. Sur ce total, huit start ups seulement ont abandonné. Les compétences se concentrent actuellement sur les mesures et asservissement (fondamentales notamment dans le domaine des éco-industries), les technologies au laser, de même que les TIC. Les partenaires institutionnels de la fabrique sont le Land, sa banque publique L-Bank et la CCI, qui apportent financement, conseil et formation. S'y ajoutent les liens avec diverses fédérations professionnelles, des réseaux de créateurs d'entreprise ou de développeurs technologiques. La fabrique travaille en coopération avec les établissements d'enseignement supérieur de Karlsruhe comme avec trois instituts du réseau Fraunhofer : l'ICT (technologies de la chimie), l'IITB (informatique) et l'ISI (innovations systèmes).

\section{Politique structurelle : une action placée au service des acteurs économiques}

Le 'partage du travail' entre ces deux catégories de zones de compétitivité se nourrit d'une longue tradition manufacturière, ancrée dans un tissu très dense d'entreprises du Mittelstand particulièrement réactives à l'évolution du marché, et rompues dans l'art d'établir partenariats et réseaux. "Les clusters sont souvent des unités géographiques de petite taille, nées spontanément ", surtout dans l'industrie métallurgique et électrotechnique (Lichtblau et al., 2005). La part dominante réservée dans la structure des activités du Land à ces branches très internationalisées a assurément joué un rôle déterminant dans l'ouverture à l'international de l'ensemble des activités, de même qu'elle entretient un climat favorable à l'innovation incrémentale dans ses réseaux de sous-traitance qui rayonnent quant à eux dans les segments connexes, notamment les services. Dans ce contexte, la politique structurelle du Land a consisté à réformer en profondeur son administration (compétences exclusives des Länder) : débureaucratisation, mise en réseau ('guichets uniques') des acteurs institutionnels auparavant 
très dispersés et cloisonnés, réforme des missions de l'administration publique et introduction du management qualité.

\section{Le dispositif de transfert et de recherche appliquée du Bade-Wurtemberg}

- 7 centres de recherche indépendants placés auprès des universités. Ils ont été créés dans les années 1980. Leur vocation est d'assurer le transfert des savoirs entre la recherche universitaire et l'industrie, via projets ou réseaux de coopération. Leurs directeurs sont employés de l'université auprès de laquelle est placé leur centre. Ces centres sont détenus par des fondations et soutenus par plus de 200 entreprises. Budget 2003 : plus de 49 millions $€$ au total ; le financement privé (entreprises) s'est élevé à 16 millions $€$. Il s'agit du Forschungszentrum Informatik (Karlsruhe), du Naturwissenschaftliches und Medizinisches Institut (Tübingen), du Forschungsinstitut für angewandte Wissensverarbeitung (Ulm), de l'Institut für Lasertechnologien in der Medizin und Messtechnik (Ulm), de l'Institut für Mikroelektronik (Stuttgart), du Zentrum Fertigungstechnik (Stuttgart) et du Zentrum für Sonnenenergie- und Wasserstoff-Forschung (Stuttgart et Ulm).

- 8 instituts de recherche professionnelle de branche. Ils ont été fondés par les fédérations industrielles et/ou sont soutenus par des associations de fondations avec pour mission d'accompagner les PME dans leur modernisation technique. Ceux du Land sont spécialisées dans les domaines suivants: textile (notamment technologies de production et chimie), chimie des métaux, pigments, microtechnologies, mécanique de précision/horlogerie et tannerie. S'y ajoute à Stuttgart la succursale du Süddeutsches Kunststoffzentrum Würzburg (SKZ, matières plastiques). Budget 2003: 31 millions $€$; 10 millions de financement privé (projets).

- 14 Instituts Fraunhofer (sur les 58 d'Allemagne). Ces instituts de recherche appliquée réputés sont financés conjointement par le Bund $(90 \%)$ et le Land où ils sont implantés (10\%). Dans le Bade-Wurtemberg, leur budget total s'élevait à 227 millions $€$ en 2003 (recettes propres : 72 millions $€$ ). Leurs spécialisations : technologies de la construction (IBP, Stuttgart; IRB, Stuttgart), de l'énergie (ISE, Fribourg), TIC (IITB, Karlsruhe), robotique (IPA, Stuttgart), mesures et technologie des capteurs (EMI, Fribourg; IPM, Fribourg), ingénierie et process (IAO, Stuttgart ; ICT, Pfinztal ; IGB, Stuttgart ; ISI, Karlsruhe ; TEG, Stuttgart), matériaux (ISC, Wertheim ; IWM, Fribourg).

- 2 centres du réseau Helmoltz spécialisé dans la recherche fondamentale et le transfert. Il pilote les grands programmes fédéraux d'innovation (technologies-clefs, énergie, sciences de la terre et de l'environnement, santé et structure de la matière). Leur financement est lui aussi conjoint; le Land y contribue à hauteur de $10 \%$. Budget total 2003 : 350 millions $€$ (dont 19 millions $€$ de ressources propres). Les deux centres installés dans le Bade-Wurtemberg sont : le Deutsches Zentrum für Luft- und Raumfahrt (DLR) avec deux sites, à Stuttgart (matériaux, énergie) et à Lampoldshausen (propulsion); le Forschungszentrum Karlsruhe in der Helmholtz-Gemeinschaft (environnement, énergie, technologies des microsystèmes).

- La Fondation Steinbeis (Stuttgart) et son réseau de centres de transfert. Elle a été créée en 1971 avec pour mission d'accompagner le Mittelstand dans le processus de globalisation des activités et, ce faisant, de soutenir à son niveau la politique de compétitivité du Bade-Wurtemberg. Cette interaction s'est concrétisée en 1982 par la création d'un poste de Chargé du gouvernement pour le transfert des technologies ; celui-ci assume en outre depuis 1983 les fonctions de président du directoire de la Fondation. Le réseau des 300 centres Steinbeis est réparti sur l'ensemble du territoire du Land, généralement à proximité des universités, où sont recrutés quelque 3030 enseignants et chercheurs (toutes disciplines). L'éventail des activités du réseau s'étend de la diffusion de l'information sur le développement des techniques et technologies ou l'élaboration d'études de marché au management et/ou pilotage de projet, sans oublier l'intelligence économique (Bourgeois, 2005). Les recettes issues des activités sur contrat se sont élevées à 89 millions $€$ en 2003.

L'existence d'une longue réputation universitaire (le Land compte 9 universités ; celle de Fribourg fête ses 550 ans en 2007), de même que la multiplication des centres de recherche appliquée du Mittelstand, constituent un autre facteur de compétitivité potentielle dont le Land a su saisir l'enjeu stratégique en développant les passerelles entre la recherche et le marché, comme le rappelle une étude réalisée par la Fondation Bertelsmann. La réforme du financement des universités intervenue en 2002 en Allemagne a été bénéfique à celles du 
Land. La part des financements publics étant désormais fixée au prorata des ressources propres (Drittmittel) de chacun des établissements, les universités bade-wurtembergeoises battent un record depuis 2002 : la part des ressources propres par poste de professeur y est de $103000 €$, contre $80000 €$ seulement en moyenne allemande. L'attractivité du site explique la présence d'une population hautement qualifiée : près d'un dixième des citoyens du Land est diplômé de l'enseignement supérieur. Au plan de la formation initiale, le système scolaire du Land (les Länder sont souverains en la matière) est considéré comme l'un des plus performants d'Allemagne dans les études PISA de l'OCDE ou leurs équivalents allemands. Conjugué à la prédominance du Mittelstand où se recrute l'essentiel des entreprises dispensant une formation professionnelle, cet état de fait explique que le taux de chômage du Land (7\%) est le plus faible d'Allemagne, quelle que soit la cohorte considérée.

La seule ressource naturelle notable du Bade-Wurtemberg étant son capital humain (et la légendaire inventivité de ses habitants), le gouvernement du Land a misé très tôt sur une politique de soutien aux approches innovantes des entreprises sur son territoire. Sa politique se contente de soutenir budgétairement les nombreuses structures de recherche appliquée et de transfert technologique ou de savoirs installées dans le Land. Elle décline certes à l'échelon régional les axes de la politique d'innovation et de recherche du Bund tout en l'insérant dans le champ européen. Mais elle suit plus encore ses orientations propres, déterminées avant tout par la structure économique bade-wurtembergeoise et les impératifs d'innovation auxquelles sont soumises ses branches dans la compétition mondiale. Dans ce contexte, le ministre de l'Economie du Land définit ainsi sa mission prioritaire : "soutenir les efforts conjoints entrepris par le monde de l'entreprise et le monde de la recherche pour développer des solutions innovantes orientées sur l'entreprise ».

Sa politique se résume dès lors à créer les conditions visant le bon fonctionnement d'un système intégré d'interaction entre l'offre en matière de recherche et de technologies et la circulation des savoirs entre tous les acteurs (y compris publics) grâce au transfert. C'est ainsi qu'il soutient la compétitivité du site de production : en contribuant au financement de tous ces établissements de recherche qui jouent le rôle de passerelle entre la recherche fondamentale (universités, Instituts Max-Planck) et le marché, et qui figurent, avec les fédérations professionnelles et les partenaires sociaux, parmi ses partenaires privilégiés. En 2006, le budget du Land consacré à ces centres et, plus généralement, à la recherche (hors établissements d'enseignement supérieur), s'élevait à quelque 240 millions $€$.

La politique structurelle du Bade-Wurtemberg, établie dans la continuité, se limite au fond à aider les acteurs économiques du Land à développer leur potentiel propre en se fondant sur un système comme une culture d'activités et d'innovation en réseau. Dans cette approche, qui inclut le développement du public private partnership, le gouvernement se situe lui-même dans une logique de partenariat avec l'ensemble des acteurs économiques comme de la société civile et, cela va sans dire, avec le réseau des 12 CCI. Son rôle est dès lors de mettre à leur disposition les moyens leur permettant d'optimiser leur propre dynamique de croissance. Ces moyens sont certes budgétaires (113 millions $€$ pour le soutien au Mittelstand en 2005), et ils se cumulent avec les prêts consentis par la L-Bank au titre du soutien à la création ou la succession d'entreprises (1,5 milliard $€$ en 2004). Ils concernent aussi l'aménagement du territoire, comme la construction d'un nouveau site pour la foire de Stuttgart, qui ouvrira ses portes à l'automne 2007, ou le développement des infrastructures de transport (conjointement avec le Bund, compétent pour les grands axes nationaux), vital 
pour la compétitivité d'une économie aussi ouverte. Le Land coopère avec la Suisse et l'Autriche pour le développement du réseau de la région du Lac de Constance, et a attendu avec impatience l'achèvement de la liaison TGV Paris-Stuttgart via Karlsruhe (s'accompagnant d'une hausse afférente de la contribution fédérale à son financement). Mais les véritables moyens de cette politique au long cours sont autres.

L'INNOVATION « NAÎT DANS UN PROCESSUS où l'économie, la science, la formation et la société agissent en réseau, et dont le centre est l'individu en tant que porteur des savoirs ». Voici comment le ministère de l'Economie du Land décrit sa nouvelle politique de compétitivité globale dans la société du savoir. Cela passe par le soutien ciblé à certains clusters. Mais il ne faut pas se méprendre sur la nature de ce soutien. "La promotion de clusters n'a rien à voir avec une politique de soutien sectoriel; il ne s'agit pas de politique industrielle sous un autre nom » (van der Linde, 2005). Il s'agit d'une approche globale dont on trouve le reflet dans les quatre thématiques développées lors du congrès de novembre 2005 des CCI du Land : mobilité et infrastructures, formation/qualification, démographie, innovation/technologies.

L'industrie du Bade-Wurtemberg n'a plus à faire la preuve de sa compétitivité. Mais les services doivent désormais prendre le relais. Quelle meilleure occasion pour en diffuser la prise de conscience que le Mondial de football dont plusieurs matches ont eu lieu dans le Land ? Diverses institutions, dont les CCI, avaient lancé à l'automne 2005 un " concours du champion du monde des services Mondial 2006 » s'adressant à toutes les entreprises et associations. Mais l'enjeu crucial pour l'avenir du site est le développement de l'exportation des services, souvent en accompagnement de la vente de produits industriels : prestations techniques, d'ingénierie, mais aussi de santé ou de loisirs. Le Land et le réseau des CCI avaient donc lancé en septembre 2005 une "Initiative pour l'exportation de services », afin de permettre aux entreprises du secteur de mieux croître à l'international et de consolider ainsi en retour l'emploi régional.

Ce nouvel axe s'insère dans une politique de compétitivité globale où le gouvernement du Land se contente généralement d'agir sur le cadre réservé aux activités et de jouer un rôle d'animateur au sein d'un ensemble de réseaux et partenariats qui se sont développés en grande partie spontanément au fil des décennies, mûs par les impératifs de compétitivité des entreprises. Voilà le véritable facteur structurel qui confère au Bade-Wurtemberg cette intelligence économique sur laquelle il a construit sa compétitivité et compte bien la développer à l'avenir.

Il s'agit là d'une culture, bien plus que de dispositifs institutionnels ou de moyens financiers (abondants et généreux au demeurant). Or cette culture, qui implique un benchmarking permanent, permet aussi de déceler les handicaps larvés et donc de développer à un stade précoce les outils permettant d'y remédier. C'est ainsi que la promotion du knowledge management fait partie des axes déclarés de la politique du Land, dans l'objectif d'une large diffusion, a fortiori dans le tissu des PME, des compétences-clefs de l'économie du savoir.

Pour mieux assurer le développement durable de la compétitivité du Bade-Wurtemberg dans l'économie du savoir, son gouvernement a institué au printemps 2007 un «Conseil de l'innovation " (Innovationsrat) chargé d'identifier les tendances stratégiques des deux décennies à venir en matière d'innovation et de conseiller le gouvernement dans son action politique. Reflet de la culture partenariale du Land, il se compose de représentants de tous les 
acteurs impliqués dans l'effort collectif d'innovation : pouvoirs publics à tous les échelons territoriaux, CCI, fédérations professionnelles, entreprises, partenaires sociaux, chercheurs et, plus largement, représentants de la société civile organisée. Son directoire comprend : le ministre-président Günther H. Oettinger, le prof. Ernst Messerschmid (Université de Stuttgart, ancien directeur du Centre des Astronautes Européens de l'ESA) et Eberhard Veit, membre du directoire de la Festo AG \& Co. KG (Esslingen), une PME spécialiste mondialement réputée de l'automatisation industrielle. Ce Conseil, dont la mission prioritaire est d'intensifier encore le transfert des technologies et savoirs entre recherche et entreprises, tout en le prolongeant dans des domaines nouveaux, est chargé notamment de développer à cet effet un High-Tech-Netzwerk Baden-Württemberg: un réseau de réseaux reliant entre eux tous ceux dédiés aux divers clusters thématiques du Land. Si le Land est fier d'être « champion du monde » à l'heure actuelle, il compte bien le rester à l'avenir...

\section{Indications bibliographiques}

ARNDT O. et al., «Baden-Württemberg - Erfolg durch Wettbewerbsfähigkeit », étude réalisée par Prognos AG pour le compte de L-Bank, 2005

Baden-Württemberg. Eine kleine politische Landeskunde, Landeszentrale für politische Bildung Baden-Württemberg, 2003 (www.lpb.bwue.de)

«Baden-Württemberg », Länderanalysen, 2005 (www.bertelsmann-stiftung.de)

Bundesländer-Ranking, étude réalisée par IW-Consult GmbH et GWS GmbH en coopération avec l'Initiative Neue Soziale Marktwirtschaft et l'hebdomadaire Wirtschaftswoche, octobre 2005 (www.iwconsult.de)

BOURGEOIS I., « Le 'miracle' biotechnologique allemand», et « Comment l'intelligence vient aux PME allemandes ", Regards sur l'économie allemande $\mathrm{n}^{\circ \mathrm{s}} 57 / 02$ et 74/05

GABEL M., «Chine, Inde, Brésil : les choix des entreprises allemandes », « Financement et vieillissement : le Mittelstand en mutation » et «Commerce extérieur allemand : faiblesses et atouts », Regards sur l'économie allemande $\mathrm{n}^{\circ \mathrm{s}} 70 / 05,69 / 04$ et 63/03

LichtBLAu K et al., «Erfolgsfaktoren von M+E-Clustern in Deutschland », IW-Trends, 2/05

Mittelstandsbericht 2005, Wirtschaftsministerium Baden-Wurttemberg, (www.wm.baden-wuerttemberg.de)

DE LA PAIX R., «Les politiques d'innovation en Alsace et dans le Pays de Bade» et « Capital-risque pour les jeunes entreprises du Bade-Wurtemberg : le rôle des pouvoirs publics ", Regards sur l'économie allemande $\mathrm{n}^{\text {os }} 54 / 01$ et $44 / 99$

VAN DER LINDE C., «Cluster und regionale Wettbewerbsfähigkeit. Wie Cluster entstehen, wirken und aufgewertet werden ", in CERNAVIN 0. et al. (eds), Cluster und Wettbewerbsfähigkeit von Regionen. Erfolgsfaktoren regionaler Wirtschaftsentwicklung, Berlin, 2005

WeInMAnN TH., «Baden-Württemberg ist die innovativste Region der EU », Statistisches Monatsheft BadenWürttemberg, 10/04

WerneR J., FisCheR B., «Europäische Metropolregionen im Vergleich», Statistisches Monatsheft BadenWürttemberg $7 / 2005$

Wirtschaftsnahe Forschungseinrichtungen in Baden-Wurttemberg, Wirtschaftsministerium Baden-Wurttemberg, 2004 (www.wm.baden-wuerttemberg.de)

www : baden-wuerttemberg.de ; bw-ihk.de ; bw-i.de ; fraunhofer.de ; steinbeis.de

Zukunftsatlas 2004, Prognos AG (www.prognos.com/zukunftsatlas). 


\title{
Hambourg : région métropole européenne et site portuaire international
}

\author{
Michèle Weinachter
}

Hambourg respire. Après l'annonce, fin février 2007, du plan « Power 8 » chez Airbus, l'avenir de la ville comme site de production de l'avionneur semble assuré. Il faut dire que la firme et l'aéronautique sont, depuis un certain temps déjà, étroitement associées à l'identité de la ville, au même titre que son port, ses docks et ses containers. La ville-Etat de Hambourg se veut la "porte qui ouvre sur le monde" (Das Tor zur Welt). Ce port maritime, de loin le plus grand d'Allemagne, lui a donné, depuis des siècles et la glorieuse époque de la Hanse, son caractère cosmopolite. L'économie du Land, et notamment le secteur de la logistique, profitent aujourd'hui pleinement de la mondialisation et des importantes mutations géopolitiques survenues en Europe depuis quinze ans. Hambourg est également un grand site des médias et des nouvelles technologies associées. Pour conforter sa position parmi les régions métropoles européennes, elle œuvre à la réalisation d'une stratégie de développement placée sous le mot d'ordre offensif : "Hambourg, une métropole en essor » (Metropole Hamburg - Wachsende Stadt). Encore devra-t-elle se donner les moyens de cette politique. *

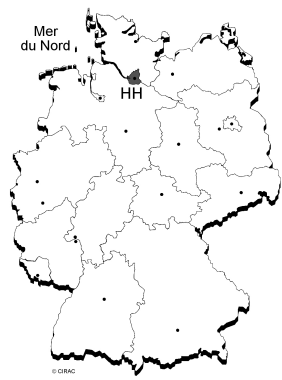

Hambourg, « ville libre et hanséatique » (Freie und Hansestadt Hamburg, $H H$ ) est située au Nord de l'Allemagne, entre les Länder de Basse-Saxe et du Schleswig-Holstein, à la confluence de l'Alster, du Bille et de l'Elbe. Comme Berlin et Brême, elle est la fois une ville et un Land: une ville-Etat (Stadtstaat). Hambourg compte 1,7 million $\mathrm{d}$ 'habitants, ce qui en fait la $2^{\mathrm{e}}$ ville allemande après Berlin, pour une superficie de $755 \mathrm{~km}^{2}$, soit 2,5 fois celle de Londres, mais avec une densité de population inférieure de moitié (2 310 hab./ $\mathrm{km}^{2}$ ).

Depuis 1991, Hambourg forme, en association avec 12 arrondissements des deux Länder voisins et deux arrondissements partenaires du Mecklembourg, qui regroupent au total près de 800 communes, la Région métropole Hambourg. C'est l'une des onze Régions métropoles européennes d'Allemagne, ces entités désormais considérées comme centrales dans le cadre de la stratégie de Lisbonne, les mises en réseau régionales devant permettre de mieux affronter la concurrence internationale. Cet ensemble structuré le long de l'Elbe compte près de 4,3 millions d'habitants. Il s'étend de Cuxhaven au nord-ouest, à l'extrémité de l'estuaire du fleuve, jusqu'aux frontières du Land de Saxe-Anhalt au sud-est, en passant par l'Elbland et la lande de Lunebourg en Basse-Saxe.

L'Elbe (1 $165 \mathrm{~km}$ de long) est, après le Rhin, le deuxième fleuve navigable d'Allemagne par son importance. Elle prend sa source en République tchèque dans les monts des Géants et se jette dans la mer du Nord à Cuxhaven après un estuaire d'une centaine de kilomètres sur

\footnotetext{
* La version initiale de cet article est parue dans Regards sur l'économie allemande, nº 81, mai 2007.
} 
lequel se trouve Hambourg. Elle possède un vaste bassin fluvial et est au centre d'un ensemble de canaux constituant un important réseau de communications entre Rhin et Baltique. La Moldau qui passe par Prague se jette elle-même dans l'Elbe, et avec la réunification de l'Europe, la circulation de marchandises sur l'axe Prague-Dresde-Magdebourg-Hambourg s'est intensifiée, contribuant à l'extension des activités du port de Hambourg.

La ville de Hambourg a été fondée au IX ${ }^{\mathrm{e}}$ siècle sous Charlemagne. Le commerce prend son essor au XIII siècle. En 1321, Hambourg devient membre de la Ligue Hanséatique, ce qui a fortement structuré son identité, comme l'indique aujourd'hui encore son nom officiel : Freie und Hansestadt Hamburg. En 1515, elle obtient le statut de «ville libre d'Empire ». Elle est alors l'une des cités les plus riches d'Europe. Depuis 1949, Hambourg est un Land autonome, dirigé depuis 7 ans par un maire CDU, Ole von Beust, qui dirige également le Gouvernement du Land, nommé « Senat ».

L'architecture se caractérise par un mélange de styles et de matières, le plus typique étant le style de construction du début du $\mathrm{XX}^{\mathrm{e}}$ en brique rouge, caractéristique du Nord de l'Allemagne, qu'on trouve dans la partie ancienne des docks. L'ensemble est marqué par la présence de l'eau, de nombreux canaux traversent Hambourg, l'Alster formant jusqu'au cœur de la ville un lac artificiel. C'est surtout le développement de son célèbre port au bord de l'Elbe, à $110 \mathrm{~km}$ de la Mer du Nord, qui marque l'identité de la ville.

C'est grâce à ce dernier que la ville est devenue très tôt un site commercial international. Après New-York, Hambourg est la ville qui détient le plus grand nombre de consulats (près d'une centaine). Elle est aussi le siège du Tribunal maritime international des Nations-Unies. Le port de Hambourg est le $2^{\mathrm{e}}$ port maritime d'Europe après Rotterdam. Chaque année, plus de 12000 navires y jettent l'ancre. Avec ses $87 \mathrm{~km}^{2}$, il occupe un dixième de la surface de la ville. Près de 140000 emplois en dépendent directement ou indirectement.

\section{Une économie diversifiée}

Si le port conserve un rôle central dans l'économie de la ville et de la région, d'autres activités se sont largement développées, notamment dans le domaine de la logistique, de la technologie, des médias, et des services. Une économie moderne, qui génère plus d'un million d'emplois, avec un PIB de 86 milliards $€$ en 2006 (3,73 \% du PIB allemand). C'est plus que Berlin (80,3 milliards $€$ ) qui compte pourtant deux fois plus d'habitants. Parmi les 500 entreprises qui génèrent le plus fort $\mathrm{CA}$ en Allemagne, 39 ont leur siège à Hambourg. A côté des grands noms comme Airbus, Beiersdorf, Hapag-Lloyd, Helm, Olympus, Otto Versand, Panasonic, Tchibo ou de grands groupes de presse comme Gruner + Jahr ou Bauer, Hambourg compte également de nombreuses PME performantes sur le marché international.

Création de richesse par secteurs en 2006 (en milliards $€$ )

\begin{tabular}{|lcc|}
\hline & Hambourg & Allemagne \\
Agri-, sylviculture, pêche & $0,14(0,2 \%)$ & $20,09(1,0 \%)$ \\
Industrie (dont BTP) & $14,18(18,3 \%)$ & $622,03(29,9 \%)$ \\
Commerce, hôtellerie, transports & $21,26(27,4 \%)$ & $380,41(18,3 \%)$ \\
Autres services & $42,04(54,1 \%)$ & $1056,47(50,8 \%)$ \\
Total & 77,63 & 2079,00 \\
\hline
\end{tabular}

Source des données : Statistische Ämter des Bundes und der Länder ; pourcentages calculés par nos soins et arrondis.

Même si aujourd'hui, moins d'un actif sur six travaille dans le secteur secondaire, l'industrie reste, ici comme ailleurs, un moteur de développement pour les autres secteurs, en particulier 
celui des services, et pour l'innovation technologique. Hamburg demeure, avec Munich et Stuttgart, l'une des grandes métropoles industrielles de l'Allemagne. Le nombre d'emplois dans ce secteur a même augmenté ces dernières années pour certaines activités, comme l'aéronautique. Les biens d'investissement représentent $33 \%$ de la production industrielle, viennent ensuite le bâtiment ( $22 \%$ ), les biens de production, l'aéronautique (10\%), l'agroalimentaire (8\%) et les biens de consommation (7\%).

Répartition des actifs par secteur en 2005 (en milliers)

\begin{tabular}{|lcc|}
\hline & Hambourg & Allemagne \\
Agri-, sylviculture, pêche & $6(0,8 \%)$ & $867(2,4 \%)$ \\
Industrie (dont BTP) & $145(18,1 \%)$ & $10869(29,7 \%)$ \\
Commerce, hôtellerie, transports & $238(29,7 \%)$ & $8504(23,3 \%)$ \\
Autres services & $413(51,5 \%)$ & $16326(44,6 \%)$ \\
Total & 802 & 36566 \\
\hline
\end{tabular}

Source des données : Statistische Ämter des Bundes und der Länder; pourcentages calculés par nos soins et arrondis.

Historiquement, l'industrie de Hambourg s'est tout d'abord développée en lien avec les activités portuaires : importation et transformation de matières premières, pour le marché intérieur ou pour l'export. Les entreprises industrielles liées aux activités maritimes, comme la construction de bateaux, de machines ou de moteurs, l'électrotechnique et le bâtiment constituent, aujourd'hui encore, le deuxième pilier de l'industrie hambourgeoise. Un troisième pilier s'est développé, celui des activités non liées au port, comme celui des biens de consommation, grâce à la demande d'une population croissante dans la région. Plus récemment enfin se développe un réseau de PME dans le secteur des éco-industries, en forte croissance.

Plus de trois quarts des actifs travaillent dans le secteur des services. Le commerce et la logistique y occupent une place centrale, avec 40000 entreprises et 200000 emplois. Ils font eux-mêmes appel à des services associés à leurs activités, comme les banques et assurances. C'est à Hambourg que la première bourse allemande avait ouvert ses portes en 1558, la première banque privée en 1590, et la première compagnie d'assurance en 1676. Aujourd'hui, la ville occupe la deuxième place derrière Francfort pour le nombre de banques (160), avec 30000 employés. Pour les assurances, Hambourg est numéro 3 en Allemagne (20 000 emplois), certains assureurs étant spécialisés dans le transport de marchandises, comme la Junge \& Co. Versicherungsmakler GmbH.

Le développement économique de la ville a été depuis vingt ans largement dynamisé par l'évolution de la situation internationale. Depuis des siècles, Hambourg constitue pour les marchandises en provenance de tous les continents la porte d'entrée vers les marchés européens. Depuis 1986, la ville est jumelée avec Shanghai, le plus grand port de commerce chinois. L'accélération de la globalisation a donné à Hambourg une forte dynamique de croissance. L'évolution de la situation géopolitique européenne à la fin du $\mathrm{XX}^{\mathrm{e}}$ siècle a joué dans le même sens. Depuis l'intégration dans l'UE, en 1995, de la Finlande et de la Suède, l'ensemble des pays scandinaves étaient désormais dans l'Union. L'élargissement à l'est en 2004 et 2007 a encore un peu plus étendu le grand marché unique. Hambourg a pu développer ou renouer des liens parfois très anciens avec les pays d'Europe centrale d'une part, avec toute la zone de la Mer Baltique 'réunifiée', d'autre part - une zone qui connaît une forte croissance économique et constitue un marché attractif de plus de 75 millions d'habitants. 
Principaux partenaires commerciaux de Hambourg en 2005 (en milliards €)

\begin{tabular}{|lcc|}
\hline & Exportations & Importations \\
UE & $15,41(67,8 \%)$ & $22,16(48,4 \%)$ \\
Continent américain & $2,83(12,5 \%)$ & $7,51(16,4 \%)$ \\
Total & $2,77(12,2 \%)$ & $10,43(22,8 \%)$ \\
\hline
\end{tabular}

Source des données : Statistische Ämter des Bundes und der Länder; pourcentages calculés par nos soins et arrondis.

Le commerce extérieur se développe en conséquence. En 2005, les importations ont augmenté de $10,5 \%$, et les exportations de $8,3 \%$. Les pays d'Asie sont devenus l'un des partenaires les plus importants de Hambourg. Le commerce avec la Chine notamment se développe de façon exponentielle (la croissance des exportations vers la Chine a été de $67,2 \%$ en 2005 , atteignant un total de 1,55 milliard $€$ ). Mais en volume, l'échange de marchandises à l'intérieur de l'Union européenne occupe, de loin, la première place, et il va continuer de croître avec le développement des nouveaux Etats membres. Enfin, le continent américain est également un partenaire important.

\section{Trois piliers de développement économique}

Cette évolution de la situation internationale a permis à Hambourg de conforter sa place en tant que pôle leader dans le domaine de la logistique pour l'ensemble de l'Europe du Nord. La logistique est en effet, à côté de l'aéronautique et de la branche MITT (médias, technologies de l'information, télécommunications), l'un des trois piliers du développement économique de la métropole hanséatique.

Dans le domaine de la logistique, le port de Hambourg se classe numéro deux en Europe, et $8^{\mathrm{e}}$ au plan mondial. En 2006, avec une croissance de 9,6\%, de nouveaux records ont été battus : plus de 135 millions de tonnes de marchandises, soit 8,9 millions de conteneurs (TEU) ont transité par le port. Sur ce total, plus de la moitié (4,8 millions) concerne le commerce avec l'Asie. De fait, la Chine est désormais le principal partenaire commercial de Hambourg, où sont installées plus de firmes chinoises (désormais plus de 400) que dans n'importe quelle autre ville d'Europe continentale. Le site Internet du port est accessible en allemand, anglais, polonais, russe, tchèque et chinois, ce qui est significatif de l'évolution en cours (www.hamburg-hafen.de). Plus d'un tiers des marchandises débarquées sur le port a pour destination finale l'une des communes de la Région métropole, où 230000 actifs travaillent dans le secteur de la logistique, dont 154000 à Hambourg même. Ils génèrent $11 \%$ de la création de richesse de la ville-Etat.

Les marchandises peuvent, après leur arrivée à Hambourg, être acheminées vers leur destination par voie fluviale, ferroviaire, routière et aérienne. Ces différents modes de transport se livrent d'ailleurs une rude concurrence. Le fret ferroviaire détient actuellement $70 \%$ du marché, le port de Hambourg étant l'un des principaux clients de la Deutsche Bahn. Il faut dire que Hambourg est le principal point d'interconnexion ferroviaire du Nord de l'Allemagne : cinq grandes lignes et deux lignes régionales s'y croisent, assurant des liaisons fréquentes avec Berlin, Hanovre, Brême/la Ruhr, Flensburg/le Jütland, Lübeck/Copenhague et, au-delà, avec les principaux centres économiques européens. 
La région bénéficie également d'un excellent maillage de canaux reliant entre eux les fleuves et les mers. L'Elbe-Seiten-Kanal $(115 \mathrm{~km})$ notamment permet de relier Hambourg à l'ensemble du réseau fluvial allemand. En l'empruntant, les bateaux de fort tonnage ont accès au Mittellandkanal $(326 \mathrm{~km})$, le plus grand canal transversal d'Allemagne. Le Nord-OstseeKanal $(99 \mathrm{~km})$ relie pour sa part la mer du Nord à la Baltique. Le transport routier devrait quant à lui se développer à la faveur de l'amélioration prévue du réseau routier et autoroutier de Hambourg, considéré comme vieilli et insuffisamment développé. Hambourg compte néanmoins plus de 1700 entreprises de transport routier. Le fret aérien dispose pour sa part d'infrastructures ultra-modernes, comme d'une grande proximité avec le centre-ville $(8 \mathrm{~km})$.

Pour conforter la place de Hambourg dans ce secteur (qui devrait, d'ici 2015, créer environ 15000 emplois supplémentaires) a été créée en 2006 la Logistik-Initiative Hamburg. Ce réseau qui regroupe les acteurs de la branche et les pouvoirs publics cherche à en accroître la compétitivité, en favorisant notamment la synergie avec les instituts de recherche et de formation. Hambourg propose ainsi de nombreuses formations dans le domaine de la logistique, y compris un MBA à la Hamburg School of Logistics, afin de fournir aux entreprises la main d'œuvre dont elles ont besoin.

En Allemagne du nord, un cluster regroupant plus de 1900 entreprises et institutions s'est formé dans le domaine de l'aéronautique civile. Il a pour centre Hambourg qui est, après Seattle et Toulouse, le troisième plus grand site de production dans le monde (les activités de Munich, premier pôle allemand de l'industrie aéronautique et spatiale, sont plus diversifiées). 32000 personnes travaillent dans cette branche. Les trois plus gros employeurs sont Airbus Deutschland GmbH, la Lufthansa Technik AG, filiale de la Lufthansa, et l'aéroport de Hambourg. Ils travaillent en collaboration avec leurs fournisseurs, plus de 300 PME du secteur qui emploient près de 9000 personnes dans la région. Au-delà de la construction, de la maintenance et de l'exploitation des avions, cette branche génère de nombreux emplois dans les services associés : ingénierie, conseil, design, ou encore dans l'électrotechnique, l'électronique, l'optique, la construction mécanique et le bâtiment. Les grandes entreprises du secteur ont établi des liens étroits avec certaines institutions de formation supérieure et de recherche, comme Airbus avec l'Université Technique de Hamburg-Harburg (TUHH), ou encore la Lufthansa Technik Logistik avec l'Institut Fraunhofer IIS (Institut für Integrierte Schaltungen, Institut Fraunhofer pour les circuits intégrés).

Ici encore, il s'agit de maintenir la compétitivité et de favoriser recherche et innovation. Hambourg est par exemple l'un des principaux pôles allemands de recherche sur la filière hydrogène et ses applications dans l'aviation et la marine (Bourgeois, 2004). La ville soutient activement ces coopérations dans le cadre de l'Initiative Luftfahrtstandort Hamburg (Hambourg, site de production pour l'aéronautique), lancée en 2001.

La société Flughafen Hamburg GmbH est exploitant de l'aéroport de la ville. Au total, celui-ci emploie plus de 12000 personnes. La capacité de l'aéroport, situé en zone urbaine, reste limitée, ce qui est considéré comme un handicap pour l'économie de la ville. Il a cependant battu des records en 2006, où il a enregistré à la fois sa plus forte croissance et un nombre de passagers encore jamais atteint auparavant: avec 11,95 millions de passagers, il est désormais le $4^{\mathrm{e}}$ aéroport d'Allemagne, et dessert près de 120 destinations. Un programme de modernisation et d'extension est en cours de réalisation. Ce projet, HAM 21, qui prévoit plus de 350 millions $€$ d'investissements, est le plus important depuis la création de l'aéroport. Il prévoit la construction d'un nouveau terminal, d'un parking de 2200 places, d'une nouvelle 
galerie commerciale, et l'amélioration des voies d'accès à l'aéroport, avec notamment un raccordement au réseau de trains rapides de la ville. Le nouveau tronçon permettra de rejoindre la gare du centre-ville en 23 minutes. L'aéroport devra ainsi pouvoir accueillir jusqu'à 15 millions de passagers par an. L'aéroport héberge également la Lufthansa Technik AG, filiale de la Lufthansa AG. Elle emploie plus de 7000 personnes à Hambourg. Avec un CA de plus de 3 milliards $€$ par an, elle est la plus grosse entreprise de maintenance, réparation et révision d'avions au monde, au service de 340 compagnies aériennes.

Mais l'élément le plus dynamique de ce cluster, et son plus gros employeur, est Airbus. Hambourg est en effet le plus gros site de production de l'avionneur outre-Rhin. Dès 1985, Hambourg comptait $20 \%$ des employés de la compagnie. Sur les 52000 salariés du groupe, 12500 travaillent désormais à l'usine de Hamburg-Finkenwerder. C'est à Hambourg que se fait depuis 1993 l'assemblage final des modèles A318, A319, A321. Hambourg est également associé à la construction du nouvel avion géant $\mathrm{A} 380$, avec notamment la production de certaines parties du fuselage et l'équipement des cabines. Depuis plusieurs années, les pouvoirs publics ont investi près d'un milliard d'euros dans un vaste programme de développement des infrastructures liées à la production et à la livraison de l'A380. Le prolongement de la piste de décollage, contre lequel les écologistes avaient livré un long combat acharné, sera finalement achevé courant 2007. Depuis 2000, près de 4000 emplois ont été créés.

\section{POWER 8 préserve le site de production de Hambourg}

Dans ce contexte, l'annonce depuis l'automne 2006 d'un vaste plan de restructuration chez l'avionneur, dû notamment au retard pris dans la production de l'A380, ne pouvait que susciter de vives inquiétudes. 10000 suppressions d'emplois étaient envisagées, la vente de certains sites, la "recherche de partenariats » pour d'autres. Finalement, le plan Power 8, dévoilé le 28 février dernier, a été accueilli avec un certain soulagement à Hambourg. Certes, ici aussi, de nombreux emplois seront supprimés (plus de 1000 sur les 3700 suppressions de postes prévues en Allemagne)

Contrairement à d'autres sites (Basse-Saxe ou Bade-Wurtemberg), Hambourg n'est pas remis en cause comme site de production. Le Senat de la ville estime qu'à moyen et long terme, il se trouve même renforcé. Hambourg continuera notamment, ce qui est perçu comme décisif, à participer au développement des produits Airbus, et tous les engagements pris antérieurement par EADS seront tenus. L'usine de Finkenwerder reste chargée du fuselage et de certains équipements de l'A380, ainsi que de l'assemblage des A318, A319 et A321. De plus, Hambourg obtient immédiatement une troisième ligne de montage pour le modèle A320 (qui était jusque-là exclusivement assemblé à Toulouse). La nouvelle version de l'A320 sera également confiée à Hambourg. L'obtention de la troisième ligne de montage pour l'A320 a été parfois présentée comme une concession faite à Hambourg " en échange » de la concentration de l'assemblage de l'A350 sur le site de Toulouse. En réalité, cette décision avait été prise dès 2000, mais la mise en œuvre avait jusqu'alors été différée. Hambourg a pu sortir ce 'joker' lors des négociations autour de Power 8.

Le scénario-catastrophe redouté par certains a donc été évité, et Hambourg restera, à côté de Toulouse, le principal site de production d'Airbus. Le Senat de la ville-Etat veut y voir aussi le résultat de son action de soutien en faveur du groupe: outre les investissements évoqués ci-dessus, Hambourg s'est engagé financièrement dans le groupe EADS lors du récent retrait de DaimlerChrysler, qui a cédé 7,5\% du capital. Hambourg est désormais le Land le plus engagé financièrement dans EADS. Mais Airbus n'a pas encore surmonté la crise actuelle, et les inquiétudes quant à l'avenir du groupe européen demeurent.

La branche des médias, des TIC et des télécommunications constitue le $3^{\mathrm{e}}$ pôle de développement du Land. Hambourg est la capitale allemande de la presse depuis près de quatre siècles. Son premier hebdomadaire a paru en 1618. Aujourd'hui, deux des quatre plus grandes maisons de presse ont leur siège à Hambourg : Gruner + Jahr (filiale de Bertelsmann, Gütersloh) et le groupe Bauer. S'y ajoute le siège central de la rédaction du quotidien à fort tirage, Bild, publié par les éditions Axel Springer (Berlin). Ensemble, ces trois groupes 
détiennent près de $50 \%$ du marché de la presse grand public. C'est ici aussi qu'est produit l'hebdomadaire Der Spiegel. C'est également à Hambourg qu'est installée la plus grande agence de presse allemande, la dpa (Deutsche Presse Agentur).

Mais le monde des médias ne se résume pas aux journaux, à la télévision et Internet, même si ces trois branches comptent près de 6000 entreprises. Il implique de plus en plus d'acteurs, par exemple dans la publicité (secteur où Hambourg fait figure de 'capitale' allemande), le design ou le marketing. Hambourg incarne la complexité de ce secteur en pleine croissance : la ville compte plus de 11000 entreprises dans le monde des médias, soit $75 \%$ de plus qu'en 1995. Le secteur (plus de 70000 actifs) est le $4^{\mathrm{e}}$ plus gros employeur de la ville et génère un CA de plus de 25 milliards $€$ ( $3^{\mathrm{e}}$ position dans l'économie de la ville). Mais on arrive à près de 17000 entreprises et 120000 actifs si on ajoute le domaine des télécommunications et des technologies de l'information, qui fournit matériel et services pour les nouveaux médias numériques et les nouvelles techniques de communication. $\mathrm{Ce}$ secteur, au CA de 9 milliards $€$ en 2005, devrait continuer à connaître une forte croissance. Le Senat (gouvernement) soutient activement cette branche, et s'est fixé pour objectif de développer à Hambourg un "Kompetenz-Cluster IT» (pôle de compétitivité TIC), dans le cadre de la stratégie globale de développement de la ville présentée en 2002.

Cette stratégie à moyen et long terme, baptisée "Metropole Hamburg - Wachsende Stadt » : Hambourg, une métropole qui croît, vise à conforter la position de la région parmi les grandes métropoles européennes et internationales. Elle a défini quatre objectifs principaux, quantitatifs mais aussi qualitatifs : une croissance durablement supérieure à celle des villes comparables, l'augmentation du nombre d'habitants, une plus forte attractivité et le renforcement du rôle de métropole, la préservation de la qualité de vie dans le cadre d'un développement durable. Du point de vue économique, cela se traduit par un certain nombre de priorités : un soutien actif aux 120000 PME de la Région métropole; une politique de cluster dans les secteurs innovants et/ou à forte croissance ; une amélioration de la coopération entre les différentes instances de la Région métropole en vue de soutenir la croissance ; enfin, la réalisation du grand projet d'urbanisme « Hafencity » (cité portuaire).

\section{La Hafencity, emblème de l'expansion de la ville}

Ce projet futuriste, le plus grand chantier urbain en Europe, va se traduire par une extension de surface de $40 \%$ (voir www.hafencity.com). L'achèvement des travaux (5 milliards $€$ d'investissements) est prévu pour 2020. La Hafencity mêlera sur 155 hectares logements, bureaux, centre de foires et de congrès, activités économiques dans tous les secteurs (40 000 emplois). Hambourg entend ainsi attirer de nombreuses entreprises ; SAP, Jungheinrich ou Unilever ont déjà annoncé leur installation.

La Hafencity se veut l'incarnation du dynamisme de Hambourg et de son économie. Son rayonnement passera également par une offre culturelle et de loisirs remarquable : outre plusieurs musées et un aquarium, la Hafencity accueillera l'Elbphilarmonie, censée devenir l'une des dix meilleures salles de concert du monde. Son coût, objet de polémiques, est finalement estimé à 240 millions $€$, soit 50 millions de plus que prévu. Le bâtiment sera en partie financé par des dons des citoyens, d'entreprises et de fondations (65 millions $€$ ont déjà été récoltés). Les travaux de construction ont commencé début avril 2007. Son achèvement est prévu pour mai 2010. L'Elbphilarmonie, à l'architecture résolument contemporaine, doit devenir le nouveau symbole de la ville-Etat.

Ces projets de développement devraient également profiter à la branche du tourisme, qui génère d'ores et déjà près de 100000 emplois directs et indirects, avec une capacité hôtelière de 30000 lits. Avec 6,4 millions de nuitées et 55 millions de visiteurs au total en 2005, 
Hambourg se classe en treizième position parmi les grandes villes européennes - devant Lisbonne, Bruxelles, Stockholm ou Copenhague. D'ici 2010, Hambourg aspire à se classer parmi les dix premières destinations européennes. Pour l'instant, le point faible reste le nombre insuffisant de touristes étrangers ; ils représentent seulement $20 \%$ du total. La ville mise ici sur la multiplication des liaisons aériennes intercontinentales directes et une meilleure desserte de Hambourg par les compagnies à bas coûts. La ville veut également conforter sa position de premier port d'accueil pour les croisières touristiques - un marché en pleine expansion. En 2006, le terminal pour bateaux de croisière a accueilli plus de 60 navires, dont le Queen Mary 2 et le MS Europa. Dans le cadre du projet Hafencity, la capacité d'accueil du Hamburg Cruise Center doit encore être améliorée.

\section{Des performances économiques contrastées}

La situation économique de la ville-Etat s'est nettement améliorée en 2006. Le Senat veut voir dans ces bons résultats économiques les premiers succès de sa politique de développement. En 2006, la croissance du PIB du Land a été remarquable. Avec $+2,9 \%$, Hambourg se classe en $3^{\mathrm{e}}$ position après la Saxe $(+4 \%)$ et le Bade-Wurtemberg $(+3,5 \%)$, la moyenne allemande se situant à $+2,5 \%$ (ces données qui datent d'avant la révision effectuée fin février 2007 par Destatis, et qui porte la croissance de la RFA à 2,9\%, n'ont pas encore été actualisées en ce qui concerne les Länder). Le chômage a connu une baisse de 13,6 \% entre février 2006 et février 2007, et le taux s'élève désormais à $10 \%$ de la population active. Hambourg a certes profité d'un contexte général favorable, marqué par le redémarrage de l'économie outre-Rhin. Parmi les évolutions encourageantes, on relève une augmentation du nombre d'actifs de $1,2 \%$ entre 2003 et 2005 . Hambourg arrive ici en $1^{\text {ère }}$ position, à en croire l'étude comparative INSM/Wirtschaftswoche Dynamik-Ranking de 2006, qui classe les Länder allemands en fonction de l'évolution d'un certain nombre d'indicateurs économiques et structurels entre 2003 et 2005 . Hambourg continue également à enregistrer de bons résultats en ce qui concerne le PIB par habitant (avec $45992 €$ par tête, il est largement au-dessus de la moyenne des 3 villes-Etats : $35463 €$ ) et la productivité (76 $084 €$ par actif; moyenne des 3 villes-Etats : $64017 €$ ).

Mais l'économie hambourgeoise n'enregistre pas que de bons résultats. Si le taux de chômage est désormais dans la moyenne allemande, il reste bien supérieur par exemple au taux de chômage de la Bavière ou du Bade-Wurtemberg (autour de $6 \%$ ), ou même à la moyenne ouest-allemande du début 2007 (8,4\%). Et au total, Hambourg n'arrive qu'en $10^{\mathrm{e}}$ position parmi l'ensemble des Länder dans le classement 2006 établi par l'étude INSM/Wirtschaftswoche Dynamik-Ranking évoquée ci-dessus. La part des dépenses publiques consacrée aux investissements a notamment chuté de 3,1\% entre 2003 et 2005. En 2005, elle n'atteignait que $10,8 \%$ du budget - la ville arrive ici en avant-dernière position pour la RFA.

Il faut dire que la ville-Etat est lourdement handicapée par le poids de sa dette : avec une dette de $12367 €$ par habitant, Hambourg fait partie du trio de tête des Länder les plus endettés d'Allemagne (Brême : $20149 €$; Berlin : $17354 €$ ). Le problème est tel que le Senat explore des pistes assez radicales pour sortir de la crise. Depuis 2005, le maire et ministreprésident Ole von Beust envisageait même de vendre la partie du Port et de ses installations appartenant à la ville, la Hamburger Hafen und Logistik AG (HHLA). Le projet avait suscité chez certains une vive indignation, tant le port est perçu comme faisant partie du patrimoine 
collectif. Après l'échec des négociations avec la Deutsche Bahn en 2006, le Senat était à la recherche d'une autre solution. Finalement, à la mi-mars 2007, l'introduction en bourse de $30 \%$ des parts de la société a été annoncée. La nouvelle a été accueillie avec soulagement notamment par les syndicats qui menaçaient de déclencher une grève et de paralyser le port. Mais le problème de la dette demeure, et tant qu'il n'est pas réglé, la marge de manœuvre de la ville pour la mise en œuvre de sa stratégie de développement s'en trouve considérablement restreinte. Cette question sera sans nul doute au centre des débats qui précéderont les échéances électorales régionales du début de 2008 (élections au Landtag).

CONCERNANT L'AMBiTION DE HAMBOURG de conforter sa place parmi les grandes métropoles européennes, les années qui viennent vont être décisives. Les axes de développement ont été clairement fixés. La dynamisation des infrastructures doit conforter les atouts territoriaux, des pôles de compétitivité émergent dans les secteurs de la technologie et de la communication, le tout contribuant au rayonnement urbain et culturel de la métropole hanséatique. Par son positionnement international, la ville-Etat profite pleinement des opportunités offertes par les élargissements récents de l'UE. Elle profite également de la globalisation et de l'essor de certaines branches qui en dépendent directement, y compris dans le domaine industriel, avec par exemple l'aéronautique. Au moment où Airbus connaît des difficultés et doit se restructurer, une embellie a semblé apparaître au plan commercial. En mars, l'avionneur européen a notamment reçu la promesse de deux commandes pour son futur long courrier A350 XWB, pour un montant record de 17,3 milliards $€$. Mais au même moment, une nouvelle menace se profile, qui pourrait bien, à relativement brève échéance, concerner Hambourg : la Chine vient de donner son feu vert au programme d'un avion gros-porteur civil susceptible de transporter plus de 150 passagers. Dans ce domaine comme dans d'autres secteurs industriels stratégiques, les Chinois vont, après avoir largement bénéficié de transferts technologiques des pays occidentaux, leur livrer une rude concurrence. Airbus, qui se partage pour l'instant avec Boeing un marché chinois en pleine croissance, a du souci à se faire. En revanche, le port de Hambourg devrait pouvoir jouer pleinement le rôle de plaque tournante du commerce euro-chinois : la Chine vient de dépasser les Etats-Unis comme premier fournisseur de produits à l'UE.

\section{Indications bibliographiques}

BERTELSMANN-STIFTUNG, Die Bundesländer im Standortwettbewerb 2005, Gütersloh, 2006

Bourgeois I., «L'enjeu de la pile à combustible et ses réseaux en Allemagne », Regards sur l'économie allemande, $\mathrm{n}^{\circ} 66$, mai 2004

Bundesländer-Ranking, étude réalisée par IW Consult en partenariat avec l'Initiative Neue Soziale Marktwirtschaft et l'hebdomadaire Wirtschaftswoche, oct. 2006 (www.insm.de)

HSH Nordbank, Hamburg im internationalen Metropolenvergleich, Coll. Regionalstudien, octobre 2003

KROPP P., « Hamburg - Wirtschaft und Arbeitsmarkt im Metropolenvergleich », in Sozialer Fortschritt, vol. 11/12, 2006

Monitor Wachsende Stadt - Bericht 2006, édité par le Statistisches Amt für Hamburg und Schleswig-Holstein, Hamburg/Kiel, 2006, en coopération avec le Senat de la ville (accessible sur le site www.wachsendestadt.hamburg.de)

www.hafen-hamburg.de ; www.hamburg-economy.de ; www.hamburg-logistik.com ; www.luftfahrtstandorthamburg.de ; www.medien.hamburg.de ; www.statistik-nord.de ; www.statistikportal.de. 



\title{
Saxe : renaissance industrielle et excellence high-tech
}

\author{
Michèle Weinachter
}

Au lendemain de la chute du Mur en 1989 - à laquelle les Saxons sont fiers d'avoir largement contribué (c'est à Leipzig qu'eurent lieu, dès le 4 septembre 1989, les " manifestations du lundi ») -, la Saxe a fait l'inventaire de ses forces. Elle ne partait pas de rien, elle qui avait été, jusqu'à la seconde Guerre mondiale, un cœur industriel de l'Allemagne. Dans de nombreux domaines, un savoir-faire ancien avait été préservé. L'Etat libre de Saxe, fort d'une identité aussi affirmée que son homologue bavarois, a cultivé et mis en valeur ses atouts grâce à une politique d'investissements ciblés au service d'une politique clairement définie : rendre le Land compétitif dans la globalisation, en misant résolument sur des créneaux d'avenir - c'est à dire sur les produits à forte valeur ajoutée. La Saxe est aujourd'hui citée en modèle. Mais une course contre la montre est engagée : les transferts financiers de l'Aufbau Ost prendront fin en 2019. D'ici là, l'économie saxonne doit consolider ses forces de croissance autonomes. *

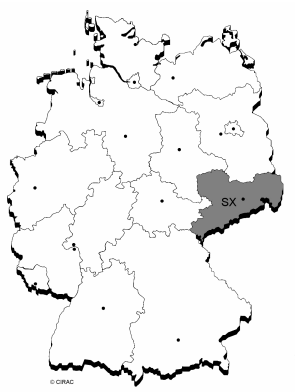

L'Etat libre de Saxe (Freistaat Sachsen; capitale : Dresde) jouxte la Pologne et la République tchèque, ce qui la place au cœur de l'UE 27. En RFA, elle a pour voisins trois autres nouveaux Länder : le Brandebourg, la Saxe-Anhalt et la Thuringe, mais aussi la puissante Bavière. Avec $5,2 \%$ de la superficie de la RFA (à peine moins que la Hesse), 5,3\% de la population (4,3 millions d'habitants) et 5,0\% des actifs, elle est un Land de taille moyenne. La Saxe n'est certes pas non plus un 'poids lourd' de l'économie allemande : elle ne 'pèse' que $3,7 \%$ du PIB allemand.

Mais son PIB (88,7 milliards $€$ en 2006, un peu plus que Hambourg) la place en tête des Länder issus de l'ex-RDA.Certes, son taux de chômage a oscillé entre $17 \%$ et $20,5 \%$ ces dernières années, pour tomber à $15,7 \%$ en 2006 sous l'effet de la reprise, à comparer à une moyenne nationale de $9,5 \%$ à la même date ; mais c'est là le tribut que continuent de payer les nouveaux Länder à la faillite de l'économie planifiée de RDA. Néanmoins, après la phase de transition et de normalisation économique, la Saxe s'est imposée comme la locomotive des Länder de l'est : de 2002 à 2004, son PIB réel a crû de 3,3\%, et après une année 2005 un peu terne où il n'a progressé que de $0,6 \%$, il a enregistré en 2006 la meilleure performance du pays avec un taux de croissance de $4 \%$, nettement supérieur à la moyenne nationale $(2,9 \%)$. Combinée à une progression record de la productivité $(+3,3 \%$ en 2006), cette vitalité la place désormais dans le peloton de tête des Länder allemands au palmarès du dynamisme régional établi chaque année par IW Consult, filiale de l'Institut der Deutschen Wirtschaft.

\footnotetext{
*Version revue et actualisée d'un article paru dans Regards sur l'économie allemande, n 77, juillet 2006.
} 


\section{Un Land qui a su renouer avec sa tradition industrielle}

La Saxe, qui est un challenger potentiel pour la Bavière, doit ce dynamisme à une politique structurelle originale et, plus encore, constante. Si le Land est gouverné depuis l'automne 2004 par une grande coalition CDU/SPD, son ministre-président, Georg Milbradt (CDU), avait été pendant dix ans le ministre des Finances de Kurt Biedenkopf (CDU), son prédécesseur à la tête du Land. Or celui-ci a joué un rôle déterminant dans les choix stratégiques de la Saxe peu après l'unité. Dès 1994, il rompt avec la politique globale et indifférenciée de l'Aufbau Ost, lance sa propre politique régionale de privatisation des entreprises de l'exRDA, mise sur le soutien ciblé au tissu historique des entreprises du Mittelstand et mène de front la modernisation du cœur historique des activités industrielles et une politique d'innovation déterminée (Bourgeois, 2001).

Structure de l'économie du Land de Saxe (2006)

\begin{tabular}{|lrr|}
\hline Part des secteurs dans la création de richesse (en \%) & Saxe & RFA \\
Agri- et sylviculture & $\mathbf{1 , 0}$ & 1,0 \\
Industrie & $\mathbf{2 3 , 9}$ & 26,0 \\
BTP & $\mathbf{5 , 9}$ & 4,0 \\
Commerce, hôtellerie, transports & $\mathbf{1 6 , 5}$ & 18,3 \\
Services aux entreprises & $\mathbf{2 6 , 7}$ & 29,0 \\
Services publics et privés & $\mathbf{2 6 , 1}$ & 21,9 \\
\hline
\end{tabular}

Sources des données: Arbeitskreis Volkswirtschatliche Gesamtrechnungen der Länder.

Si le secteur des services a presque rattrapé la moyenne allemande depuis l'unité (71,2\%des actifs en 2006), l'industrie reste le moteur de croissance de la Saxe. La production manufacturière occupe $26,6 \%$ des actifs (soit plus que la moyenne allemande : $25,5 \%$ ) ; il faut y ajouter les emplois indirects dans les services aux entreprises. Ce Land a pu relancer, en la modernisant, la production dans des secteurs où elle était déjà très développée avant la période de la RDA. La Saxe possède en effet une longue tradition industrielle. Elle doit son développement économique précoce à la richesse de ses sous-sols, notamment dans les monts métallifères de l'Erzgebirge.

Principales branches de l'industrie (2006)

\begin{tabular}{|lcc|}
\hline & CA (milliards $\epsilon$ ) & Actifs \\
Construction automobile & 12,4 & 29000 \\
Agro-alimentaire & 6,8 & 24245 \\
Bureautique, électrotech., mécanique de précision, optique & 7,05 & 34500 \\
Production et transformation des métaux & 6,4 & 39074 \\
Construction mécanique & 6,0 & 35300 \\
Papier, imprimerie, édition & 2,3 & 12783 \\
Chimie & 2,8 & 9973 \\
\hline
\end{tabular}

Sources des données: Statistisches Landesamt Sachsen.

L'industrie est concentrée dans le triangle constitué par les trois villes les plus importantes : Dresde (478 000 habitants), Leipzig (439 000 habitants) et Chemnitz (259 000) qui, du temps de la RDA, avait été rebaptisée Karl-Marx-Stadt. Avant la Seconde Guerre mondiale, cette zone était considérée comme le berceau de l'industrie allemande. Depuis longtemps, la 
Saxe était réputée pour ses inventions et la fabrication de produits à forte valeur ajoutée, basés sur l'innovation - de la découverte du secret de la fabrication de la porcelaine au début du XVIII ${ }^{\mathrm{e}}$ siècle, qui conduisit à la création de la manufacture de Meissen, à la première locomotive à vapeur. Entre 1991 et 2004, la création de richesse a connu dans la production industrielle un développement spectaculaire : elle a augmenté de $177 \%$. Aujourd'hui, les quatre moteurs de l'économie saxonne sont l'automobile, la construction mécanique, la microélectronique et les biotechnologies.

La Saxe, qui fut aussi un des berceaux de l'automobile, a développé depuis quinze ans une industrie automobile ultra-moderne, positionnée essentiellement sur le haut de gamme, et qui emploie près de 30000 personnes. La productivité y dépasse souvent celle du secteur à l'ouest, les salaires y sont inférieurs (en moyenne, de $20 \%$ ), et la durée hebdomadaire du temps de travail y est plus longue. Cette configuration la met à l'abri des rationalisations et des réductions d'effectifs programmées à l'ouest, notamment dans le groupe Volkswagen.

\section{Le renouveau de l'industrie automobile}

L'industrie automobile de Saxe a fêté en 2004 son centenaire dans la région de Chemnitz-Zwickau. C'est là que sont nées les entreprises Horch, Audi, Wanderer et Zschopauer. Elles ont fusionné en 1932 sous le nom d'Auto Union AG, fusion à l'origine des 4 anneaux de la marque Audi. C'est à Zwickau qu'était fabriquée dans les années 1920 la légendaire Horch 8 cylindres, ou la Silberpfeil 16 cylindres qui, en 1937, battit le record du monde en dépassant les $400 \mathrm{~km} / \mathrm{h}$. Avant la guerre, plus de la moitié des voitures de luxe allemandes venaient de Saxe.

Le régime communiste crée la rupture : on fabrique désormais des Trabant. Mais les savoir-faire se perpétuent. En septembre 1990, le chancelier Kohl pose la première pierre de la nouvelle usine VW de Mosel/Zwickau. De 1990 à 2003, plus de 2 millions de voitures sortent de cette usine - essentiellement des Golf, puis à partir de 1996, des Passat. 6 millions de moteurs VW sont construits dans l'usine de Chemnitz. Et depuis 2001, dans la «manufacture de verre» de Dresde, installée dans des jardins au cœur de la ville, est assemblé le modèle haut de gamme Phaeton. La modernité de cette usine entièrement transparente, accessible au public, est mise en scène (www.glaesernemanufaktur.de) selon un concept qui se place dans la tradition manufacturière de la Saxe et se veut porteur d'une nouvelle « culture automobile ».

Pour attirer de nouveaux investisseurs et soutenir dans leur développement les sous-traitants de la branche, les pouvoirs publics ont créé en 1998 l'Initiative Automobilzulieferer qui regroupe désormais plusieurs centaines d'entreprises, essentiellement dans la zone ChemnitzLeipzig-Zwikkau-Plauen (www.amz-sachsen.de). Cette démarche relève d'une stratégie globale de développement structurel menée par le Land et visant à attirer, puis à ancrer sur son territoire tous les acteurs d'une même chaîne de production. Elle favorise leur installation, crée des réseaux et des solidarités qui lient les différents maillons, y compris les laboratoires de recherche de la branche concernée, avec l'aide des CCI. Chaque acteur est volontairement impliqué dans la conception, l'élaboration dans le moindre détail des nouveaux produits, à chaque étape. Chaque maillon est ainsi lié aux autres, et devient indispensable pour la fabrication du produit fini, un produit innovant et uniquement fabriqué en Saxe - stratégie qui présente le double avantage de garantir les marchés, du moins un certain temps, pour chacune des entreprises, et de limiter les risques de délocalisation. C'est dans ce contexte que Porsche avait décidé de s'installer à Leipzig, où se trouve l'usine de montage de la Cayenne et de la Carrera GT, et où sera assemblée la Panamera - une décision qui permettra la création de 600 nouveaux emplois dans l'usine, et autant chez les équipementiers de la région. Enfin, BMW à son tour a choisi Leipzig pour produire ses modèles Série 3. 
Le second pilier du développement est celui de la construction mécanique. Il s'appuie lui aussi sur une longue tradition. La Saxe fut au XIX ${ }^{\mathrm{e}}$ siècle le berceau de la machine-outil et de la construction de machines pour l'industrie textile, internationalement réputées. Le secteur, aujourd'hui très diversifié, produit pour presque toutes les autres branches - notamment pour l'automobile. Dans le segment de la machine-outil, la Saxe compte 442 entreprises employant un peu plus de 34000 salariés (2005). Si on y ajoute celui des équipements et installations, et si on prend en compte les PME de moins de 20 salariés, ce sont près de 100000 salariés et plus de 1000 entreprises qui travaillent pour cette branche. Elles sont surtout concentrées dans la région de Chemnitz-Zwickau et le Vogtland, près de la frontière bavaroise. Le CA des 442 entreprises de plus de vingt salariés du secteur est passé de 3,3 milliards $€$ en 1997 à 5,13 milliards $€$ en 2005 . Cela ne représente que $3 \%$ du CA total de la branche en RFA, mais le secteur est en forte croissance, ce qui n'est pas le cas partout en Allemagne.

Le développement des exportations explique largement cette évolution : leur part est passée à 41,9\% du CA de la branche en 2005. Ce succès s'explique notamment par un positionnement sur des produits-niches, en particulier la production de machines-outils innovantes, par exemple dans le domaine de l'imprimerie, avec des entreprises comme Union, Xenon ou encore Planeta, leader mondial dans son domaine grâce à un nouveau procédé offset. On retrouve ici le positionnement sur le haut de gamme : le secteur ne vise pas essentiellement la production de masse, mais la qualité et l'innovation. Dans la tradition manufacturière de la Saxe, on cultive l'ultra-spécialisation et le sur mesure, pour des clients prêts à payer le prix fort. Même si la productivité n'atteint encore que $80 \%$ du niveau ouestallemand, la compétitivité du secteur est assurée par des salaires inférieurs de 20 à $30 \%$ chez les ouvriers qualifiés, et dans ce secteur aussi, la semaine de 40 heures reste la règle.

Or le secteur est le fief du Mittelstand, ce qui fait la force de l'industrie saxonne en comparaison des autres Länder de l'est, fortement désindustrialisées (sauf la Thuringe voisine). Mais les PME manquent de capitaux, la part des fonds propres ne s'élevant qu'à $30 \%$ en moyenne, contre $50 \%$ à l'ouest. Pour aider ces entreprises à travailler en réseau et compenser ainsi les handicaps liés à leur petite taille, l'Initiative Verbundinitiative Maschinenbau Sachsen (VEMAS, www.vemas-sachsen.de) a été créée fin 2003. Elle ouvre aux PME de nouveaux marchés à l'exportation, notamment en direction des PECO et de la Russie. Elle mise aussi sur le lien entre R \& D et production, en s'appuyant sur la coopération avec les établissements d'enseignement supérieur de Chemnitz et de Dresde, et les Instituts Fraunhofer. C'est ainsi qu'est né à Chemnitz l'un des trois clusters co-fondés en Allemagne par la Société Fraunhofer : l'Innovationscluster Mechatronischer Maschinenbau (mécatronique).

Troisième moteur de la croissance : la microélectronique, qui s'est développée essentiellement autour de Dresde. Du temps de la RDA, le combinat Robotron y fabriquait les premières générations d'ordinateurs. Aujourd'hui lui a succédé le plus grand cluster européen de microélectronique, et l'un des cinq premiers mondiaux. Dès les années 1990, AMD, numéro 2 mondial des microprocesseurs, et Infineon, s'y sont installés, attirés par les aides. Le Land a subventionné à hauteur de près de 1 milliard $€$ l'installation à Dresde d'AMD, qui y a localisé deux usines et vient d'annoncer la création d'un troisième pôle. Mais la possibilité de travailler avec les nombreux centres de recherches universitaires et extra-universitaires du domaine a joué un rôle plus décisif encore. Grâce à la qualité des chercheurs, $80 \%$ des activités R \& D d'AMD se font désormais en Saxe - et non plus en Californie dans la Silicon Valley, au siège du groupe à Austin (Texas), ou encore à Taïwan. 
La stratégie observée pour la construction automobile s'est avérée payante dans ce domaine aussi : attirer quelques gros investisseurs, créer des réseaux, afin d'obtenir un effet boule de neige. Plus de 200 entreprises de la microélectronique (plus de 20000 salariés) sont désormais installées en Saxe. Si on considère l'ensemble du secteur des TIC, on dénombre 1500 entreprises employant 43000 salariés et réalisant un CA annuel de 3 milliards $€$. Les acteurs du secteur sont regroupés au sein du réseau Silicon Saxony qui veille à resserrer toujours plus le lien entre recherche fondamentale et développement de projets. Dans ce contexte, l'Université Technique de Dresde, celle de Leipzig et les sept autres établissements d'enseignement supérieur concernés se sont adaptés. Les entreprises qui s'installent dans la région savent qu'elles pourront embaucher directement les jeunes diplômés, les filières de formations ayant été conçues ad hoc. De plus, ces universités signent elles-mêmes des contrats de recherche, ce qui leur permet de se financer en partie grâce aux nouvelles technologies qu'elles développent et peuvent ensuite monnayer auprès des entreprises. Silicon Saxony peut aussi s'appuyer sur la densité du réseau de centres de recherche extra-universitaires : instituts Max-Planck, Fraunhofer ou Leibniz.

Le passage de la micro- à la nanoélectronique est actuellement un enjeu de taille pour l'industrie des semi-conducteurs, qui élabore les futures générations de microprocesseurs. Le Fraunhofer-Center Nanoelektronische Technologien (CNT; www.cnt.fraunhofer.de), inauguré le 31 mai 2005 à Dresde, a été créé dans ce but. Il est installé sur le terrain de l'entreprise Infineon. Le CNT est né d'un partenariat public/privé dans lequel sont parties prenantes Infineon et AMD, mais aussi la Société Fraunhofer et l'Université Technique de Dresde. Le Bund, le Land de Saxe et l'UE ont déjà investi 80 millions $€$ dans la construction et l'équipement du CNT. 170 millions $€$ d'investissements dans des projets de recherche sont planifiés pour la période 2005-2010. C'est cette proximité sur le terrain, cette synergie entre recherche fondamentale, développement et production qui doivent permettre aux entreprises saxonnes de garder une longueur d'avance sur leurs concurrentes mondiales. La Saxe est d'ailleurs en passe de devenir l'un des premiers sites au monde pour les nanotechnologies. Plus de 80 entreprises travaillent dans ce domaine, sur 450 pour l'ensemble de l'Allemagne. Autour de Dresde, 1500 personnes travaillent dans l'un des 40 organismes de recherches sur les nanotechnologies ou dans l'une des entreprises qui les utilise. Environ 10000 emplois indirects en dépendent.

Les nanotechnologies sont également importantes pour les biotechnologies, quatrième vecteur de la croissance du Land. Plus de 150 entreprises sont impliquées, principalement dans les biotech médicales (voir www.med-in-leipzig.de). Il ne s'agit nullement d'une création ex nihilo, l'industrie pharmaceutique s'était développée en Saxe dès le XIX ${ }^{\mathrm{e}}$ siècle. Leurs activités (cellules-souches, médecine régénérative ou la mise au point de nouveaux médicaments) sont concentrées principalement autour de Dresde, où a été construit le BioInnovationsZentrum (BIOZ), et de Leipzig, où est sise la Biocity (www.bio-city-leipzig.de), deux pépinières pour les chercheurs, les start ups et les services liés. Tous sont regroupés, comme pour les autres branches dynamiques, dans un réseau : en l'occurrence Biosaxony (www.biosaxony.com). Ainsi, les infrastructures de la Biocity de Leipzig, inaugurée en 2003, accueillent actuellement 22 entreprises sur 15000 mètres carrés. Sous le même toit et c'est là l'aspect le plus innovant - 5000 mètres carrés supplémentaires sont réservés à la recherche. Ils sont occupés par 6 laboratoires de l'Université de Leipzig. Ce contexte est particulièrement favorable pour motiver les jeunes chercheurs et soutenir les start ups. Le Land 
s'est fortement impliqué là aussi, investissant plus de 200 millions $€$ entre 2000 et 2005, plus que n'importe quel autre Land.

\section{Priorité à une gestion budgétaire rigoureuse et à l'investissement productif}

C'est là l'une des clés du dynamisme de l'économie en Saxe : depuis la chute du Mur, ce Land a privilégié l'investissement productif, pour développer ses infrastructures, son système de formation et ses secteurs innovants. Cette politique est mise en œuvre de façon conséquente depuis une quinzaine d'années, alors que les autres Länder est-allemands consacrent une bonne partie des fonds de l'Aufbau Ost comme des fonds structurels européens à combler leurs trous budgétaires. Pour dégager de la marge de manœuvre en faveur de ces investissements, les pouvoirs publics ont veillé à ne pas laisser filer les déficits ; avec un endettement par habitant de $2720 €$, contre $5822 €$ en moyenne des Länder (2006), la Saxe a aujourd'hui le taux d'endettement le plus faible de toute l'Allemagne après la Bavière.

Cette politique rigoureuse se traduit dans le fait que la Saxe a désormais le plus fort taux d'investissement public de tous les Länder allemands : 23,7 \% en 2006. De 1991 à 2002, les investissements bruts en biens d'équipement s'y étaient élevés à 295,3 milliards $€$, soit le tiers de l'ensemble des investissements publics opérés dans les nouveaux Länder.

Les communes appliquent la même stratégie : en avril 2006, Dresde a vendu l'ensemble de son parc immobilier. Le produit de la vente des 48000 appartements (1,7 milliard $€)$, regroupés dans le consortium WOBA, permet à la capitale de rembourser immédiatement la totalité de ses dettes et même de placer un surplus de 100 millions $€$. Chaque année, la ville économisera ainsi 75 millions $€$ d'intérêts, ce qui lui donne les moyens de mener une véritable politique de développement économique.

Le dynamisme que connaît la Saxe aujourd'hui n'est donc pas le fruit du hasard. Au lieu de pratiquer une politique de l'arrosoir à court terme, le Land a appliqué une politique délibérée de création de pôles de compétitivité. Trois clusters ont ainsi émergé autour des villes les plus importantes : Dresde est leader pour la microélectronique, Leipzig pour l'automobile, et la région de Chemnitz/Zwickau s'est spécialisée dans la construction mécanique et la productique.

La Saxe a fait ce choix sans faire table rase du passé. Elle a au contraire renoué avec ses traditions et misé sur sa réputation dans les travaux de grande précision. Une réputation qui date d'Auguste le Fort, prince de Saxe et roi de Pologne au début du XVIII ${ }^{\mathrm{e}}$ siècle, dont la cour était, selon Voltaire, la plus brillante d'Europe après celle de Versailles. Amateur d'art et de sciences, il avait fait venir à sa cour (à Dresde) et dans ses manufactures (dont celle de Meissen) les plus grands spécialistes de toute l'Europe. Aujourd'hui, le savoir-faire est investi dans les technologies high tech.

Cette stratégie d'excellence et de positionnement sur le haut de gamme, qui vise à assurer la compétitivité du site dans la mondialisation, semble porter ses fruits : la Saxe réalise à elle seule plus du tiers des exportations des nouveaux Länder $(38,14 \%$ en 2006), et sa balance commerciale est en net excédent (6,28 milliards $€$ en 2006). Plus de la moitié (55,3\% en 2006) des exportations est destinée à des pays de l'Union européenne.

Le dynamisme du commerce extérieur n'a cessé de se renforcer depuis le milieu des années 1990. Entre 1995 et 2005, la valeur des exportations a été multipliée par 5, celle des importations par 2,75. La progression s'est poursuivie en $2006: 11 \%$ de plus pour les exporta- 
tions, toujours portées par les 'valeurs sûres' du « Made in Saxony » (automobile, construction mécanique, électrotechnique...). C’est la plus forte augmentation de ces cinq dernières années. Elles atteignent désormais 19,6 milliards $€$. Quant aux importations, elles ont augmenté de près de $25 \%$ en 2006 par rapport à l'année précédente, atteignant désormais 13,3 milliards $€$. Le Royaume-Uni est devenu le premier pays destinataire à l'intérieur de l'UE : les exportations ont progressé en 2006 de $24 \%$, essentiellement grâce à une forte progression dans l'automobile. Alors qu'en 2005, la République tchèque arrivait déjà en première position pour les importations, les exportations en direction de ce voisin immédiat ont fait en 2006 un bond de $22 \%$. Parallèlement, la Saxe participe désormais au processus de globalisation des échanges : le commerce en direction de l'Asie a pour sa part augmenté de $30 \%$ en 2006, atteignant une valeur de 3,8 milliards $€$. La Malaisie reste premier partenaire (1,2 milliard $€$ ), et les exportations en direction de la Chine ont augmenté de $51 \%(0,7$ milliard $€)$.

Cette ouverture à l'international est ancienne : la foire de Leipzig est depuis plus de 500 ans une plate-forme majeure du commerce au cœur de l'Europe. Le site correspondait au croisement des deux grandes routes commerciales de l'époque : la Via imperii dans le sens nord-sud, et la Via regia dans le sens est-ouest. Dès le $\mathrm{XV}^{\mathrm{e}}$ siècle, elle fut un lieu d'échanges majeur pour de nombreux produits, comme les pierres et métaux précieux ou la soie (la première manufacture de soie ouvrit en Saxe en 1668). C'est à Leipzig que naquit au début du $\mathrm{XX}^{\mathrm{e}}$ siècle le concept de « foire exposition ». Sous le régime communiste, la foire est restée une vitrine internationale de la RDA. Elle vient de fêter les dix ans de sa renaissance : depuis 1996, elle accueille les visiteurs dans un parc d'expositions ultramoderne, équipé d'un centre de congrès multifonctions avec services aux professionnels. La Foire du Livre et le Salon international de l'automobile (AIT) - le $2^{\mathrm{e}}$ en Allemagne après celui de Francfort - sont les plus importants parmi les 30 salons spécialisés et foires grand public qui s'y tiennent chaque année, dont nombre occupent le premier rang mondial dans leur spécialité (www.leipzigermesse.de). Chaque année, Leipzig accueille ainsi autour de 1,5 million de visiteurs.

A l'appui de sa stratégie de développement, la Saxe peut miser sur son capital humain : la qualité de sa formation est reconnue (la Saxe est classée deuxième après la Bavière par l'étude PISA de l'OCDE). La part des actifs hautement qualifiés est ici plus élevée qu'ailleurs : en 2005, la Saxe comptait 34,6 \% de diplômés de l'enseignement supérieur général ou technique et de maîtres-artisans, contre $26 \%$ en moyenne allemande. Enfin, la Saxe se classe au quatrième rang national pour la qualité de ses chercheurs. $41,4 \%$ du personnel de R \& D des nouveaux Länder travaillent en Saxe. Dans la région de Dresde, la part du PIB consacrée à la $\mathrm{R} \& \mathrm{D}$ est de 3,63\%. Même si la moyenne saxonne s'établit à 2,2 \%, (2004, dernières données disponibles), l'objectif de $3 \%$ fixé par la Stratégie de Lisbonne est déjà largement dépassé dans la plupart des bassins industriels du Land.

La réactivité de l'administration publique est une autre clé du dynamisme économique. On joue sur l'image du 'tapis rouge' déroulé aux pieds des investisseurs. La bureaucratie et les délais d'autorisation sont très réduits, et là encore règne l'esprit du sur-mesure. Un des meilleurs exemples en est sans doute le choix de la Saxe par le groupe de logistique DHL : il implante sa plate-forme européenne de transit (investissement : 300 millions $€$ ) près de l'aéroport ultra-moderne de Leipzig, ce qui devrait créer près de 3500 emplois d'ici 2012, et représente un potentiel bien supérieur encore. Pour convaincre DHL et l'emporter face à d'autres sites concurrents, les pouvoirs publics de Saxe ont notamment modifié le tracé d'une piste de l'aéroport afin de permettre le décollage et l'atterrissage simultané des avions. 
Dresde, de son côté, n'est pas en reste en matière d'attractivité, ainsi que le montre l'étude Zukunftsatlas 2007 effectuée pour le magazine Handelsblatt par l'Institut de recherche suisse Prognos. Dans cette étude qui, depuis 2004, évalue chaque année la compétitivité des villes et cantons urbains sur la base de 29 critères, et dresse un classement en fonction de leurs perspectives de développement futur, Dresde est passée en trois ans de la $110^{\mathrm{e}}$ à la $13^{\mathrm{e}}$ place, dépassant désormais Düsseldorf, Francfort sur le Main ou Hambourg.

La qualité et la modernité des infrastructures sont en outre un atout essentiel dans l'affirmation de l'excellence territoriale. Concernant les transports, Leipzig est particulièrement bien desservie, avec un aéroport international, des autoroutes et des liaisons ferroviaires ICE, à la croisée des axes Berlin/Munich et Francfort/Dresde. Tous ces équipements interconnectés sont neufs ou entièrement rénovés. Grâce à la qualité de la desserte, le vépéciste Quelle a installé à Leipzig un grand centre d'expédition, et le leader mondial de la vente en ligne Amazon projette lui aussi, au nord de la ville, la construction d'un tel centre, avec 400 emplois à la clé.

Certains projets sont encore en cours de réalisation, comme l'autoroute Dresde/Prague, un axe de communication stratégique, mais dont l'aménagement a pris du retard. Côté allemand, les travaux se sont achevés comme prévu fin 2006, mais les choses prendront plus de temps du côté tchèque, les écologistes s'opposant au tracé à travers les forêts de Bohème (Bourgeois, 2004). L'Elbe joue également un rôle majeur. Elle prend sa source en République tchèque et traverse la Saxe sur plus de $180 \mathrm{~km}$. Reliant Prague à Hambourg en passant par Dresde, l'Elbe est, après le Rhin, l'axe fluvial allemand le plus emprunté. En Saxe, plusieurs plate-formes nouvellement aménagées vont prendre de l'importance - notamment le port de Riesa au nord-est de Dresde, site intermodal permettant de combiner navigation fluviale, rail et route. Depuis 1990, la Saxe a largement profité des fonds structurels européens et, plus encore, des fonds du programme fédéral de développement des infrastructures de transport dans les nouveaux Länder (Verkehrsprojekte Deutsche Einheit), qui lui permet de disposer aujourd'hui d'infrastructures souvent plus modernes qu'à l'ouest. C'est vrai également pour d'autres types d'équipements nouvellement installés - les réseaux de télécommunication ou encore les universités et centres de recherche.

Mais pour inciter les entreprises à s'installer en Saxe, à y rester, et retenir la main d'œuvre qualifiée, le Land mise aussi sur la qualité de vie, l'offre culturelle et la diversité des activités de loisirs. En effet, si les salaires en Saxe sont inférieurs de 20 à $30 \%$ à ceux de l'Ouest, le coût de la vie y est lui aussi inférieur dans les mêmes proportions - par rapport à Munich par exemple. C'est vrai en particulier pour l'immobilier. Dresde est sans doute actuellement, avec Berlin, l'une des capitales culturelles européennes les moins chères. La ville a certes beaucoup souffert sous les bombardements en 1945, et son patrimoine historique s'est dégradé durant l'ère communiste, mais elle connaît aujourd'hui une véritable renaissance. Culture et création d'emplois y vont de pair. Dresde est actuellement la seule grande ville allemande où le tourisme connaît une croissance annuelle supérieure à $10 \%$. La vallée de l'Elbe autour de Dresde a été classée au patrimoine mondial par l'Unesco. La restauration de son patrimoine architectural est réalisée en partie à l'aide de fonds privés, comme dans le cas de la Frauenkirche, reconstruite à l'identique grâce à une vague exceptionnelle de dons répondant à «l'Appel de Dresde » lancé en 1990 : il a permis de réunir les deux tiers des 130 millions $€$ nécessaires à la restauration de cette église protestante, chef-d'oeuvre baroque, symbole des cataclysmes et du renouveau de l'histoire allemande récente. 
Mais la concentration d'activités autour de quelques pôles forts a aussi des revers, certaines régions restant à l'écart du développement, comme l'Erzgebirge ou la Sächsische Oberlausitz. Les pouvoirs publics espèrent un effet d'entraînement à partir des zones dynamiques, mais pour l'heure, la réalité dément ces attentes : les habitants de ces régions à très haut niveau de chômage émigrent, souvent vers l'ouest. L'exode touche surtout les jeunes : les deux tiers de ceux qui partent ont entre 15 et 35 ans.

Cela assombrit les perspectives démographiques. La Saxe connaît en effet, en la matière, un développement encore plus défavorable que l'ensemble de l'Allemagne. Avec le chômage et l'exode des jeunes, le vieillissement de la population s'accélère. Alors qu'au moment de la chute du Mur, la Saxe comptait près de 5 millions d'habitants, elle devrait perdre d'ici 2020 près d'un quart de sa population qui sera, de surcroît, en moyenne plus âgée que dans les autres Länder. Fin 2005, la part des jeunes de moins de 20 ans dans la population est tombée à 16,4 \% (le chiffre le plus bas de toute l'Allemagne), tandis que la part des plus de 60 ans a atteint pour la première fois $29 \%$, niveau le plus élevé du pays. Les conséquences négatives de cette évolution sur la croissance, l'emploi et les recettes fiscales sont prévisibles. Déjà, les pouvoirs publics anticipent, s'emploient à réduire les services administratifs en conséquence, et accentuent leur politique de rigueur budgétaire. L'inquiétude est présente aussi dans les entreprises. Aujourd'hui, la Saxe forme encore suffisamment de jeunes qualifiés dans les domaines où l'économie en a besoin. Mais le déficit devrait commencer à se faire ressentir d'ici une dizaine d'années. Le taux de chômage devrait alors baisser, l'exode des jeunes s'arrêter - puisqu'ils seront très demandés. Mais il sera bien tard, et cette évolution démographique est préoccupante à moyen terme. Elle entraîne le Land dans une spirale qui risque de l'affaiblir durablement, au moment même où les transferts financiers venus de l'ouest vont diminuer.

Paradoxalement, alors que l'élargissement de l'UE avait suscité bien des craintes, le salut pourrait venir de l'Est, notamment des deux voisins immédiats de la Saxe, la Pologne et la République tchèque, qui ne connaissent pas pour l'instant les mêmes problèmes démographiques. À la faveur de la mise en place progressive au sein de l'UE de la libre circulation pour les travailleurs est-europérens, les jeunes de ces pays pourraient être attirés par des salaires nettement supérieurs. Pour l'heure, en Saxe aussi, on se méfie encore du «plombier polonais ». Il est vrai que le commerce et l'artisanat ont beaucoup souffert dans les régions frontalières, et on redoute toujours des délocalisations vers les PECO, surtout pour les produits low-tech, sur lesquels il est difficile de rester compétitif à cause des différences de coûts salariaux. Pour faire face, la Saxe mise sur les atouts qui lui donnent encore un avantage compétitif sur ses voisins de l'est : une main d'œuvre mieux qualifiée, une plus forte productivité, des infrastructures ultra-modernes, un système juridique qui offre les meilleures garanties.

Cela dit, comme l'explique Alexandre de Saxe, chargé par le gouvernement du Land de promouvoir le développement économique international de la Saxe, les délocalisations ne sont pas toujours exclusivement dommageables, surtout si elles ne sont que partielles et qu'elles s'effectuent de l'autre côté de la frontière. Car elles permettront de développer facilement des coopérations, qui plus est dans le cadre de réglementations européennes. Cela vaut mieux en tout cas que de voir partir les entrepreneurs vers l'Inde ou la Chine, une stratégie souvent payante à très court terme, mais beaucoup plus hasardeuse à moyen terme à cause du risque de pillage massif de technologie - un aspect particulièrement important pour la Saxe qui se positionne essentiellement sur les produits à forte valeur ajoutée. Vu sous cet 
angle, le partage des tâches avec les nouveaux Etats membres de l'Union peut finalement apparaître comme une opportunité permettant de rester compétitif. Le positionnement du site saxon dans l'industrie automobile européenne le révèle. Un bon exemple de cette complémentarité dans la division du travail, au bénéfice de tous, est celui de Porsche. La Cayenne est fabriquée à près de $90 \%$ dans l'usine Volkswagen de Bratislava en Slovaquie, qui livre la carrosserie déjà peinte, la voiture est ensuite équipée à Leipzig, où on installe l'électronique et toutes les finitions en fonction des vœux des clients. Ici les tâches low-tech à moindre coût, là le high-tech et le sur-mesure - cela permet de conserver en Saxe un nombre non négligeable d'emplois, tout en participant à une stratégie de développement : les PECO, à force de s'enrichir en produisant moins cher, seront demain de nouveaux clients pour les produits made in Saxony.

EN ATTENDANT, UNE COURSE CONTRE LA MONTRE EST ENGAGÉE : le chômage reste à un niveau très élevé alors que la fin de l'aide financière aux nouveaux Länder est programmée pour 2019. Le budget de l'Etat de Saxe sera de plus en plus serré, et la politique d'aide aux investissements, si efficace depuis quinze ans, devra être revue à la baisse. Mais Georg Milbradt, l'actuel Ministre-Président de la Saxe, se veut optimiste : du temps de la Guerre froide, la Saxe était 'en marge'. Avec l'élargissement de l'Union européenne, elle est désormais au centre, avec à sa porte, des marchés en pleine croissance. L'axe économique dominant de l'Union, la « banane bleue » actuellement située le long de la ligne Rhin/Rhône, va probablement se décaler vers l'est, le long d'un axe allant de la Scandinavie à Budapest, en passant par Berlin, Prague et Vienne. Et donc par Dresde. Certes, la Saxe n'est pas encore uniformément devenue l'un de ces "paysages florissants » qu'annonçait imprudemment le chancelier Kohl au lendemain de l'unification. Mais elle est en train de reprendre sa place au coeur de la nouvelle Europe.

\section{Indications bibliographiques}

BerTElsmanN-STIFTUNG, Die Bundesländer im Standortwettbewerb, Gütersloh, 2005

BOURGEOIS I., " 10 ans après l'unification : l'économie des nouveaux Länder en voie de normalisation », in BOURGEOIS I. (dir.), Allemagne 2001. Regards sur une économie en mutation, CIRAC, Levallois, 2001

BOURGEOIS I., «Quelles infrastructures pour la Bohême au cœur de l'Europe ? », Regards sur l'économie allemande, $\mathrm{n}^{\circ} 66$, mai 2004

DIW / IAB / IfW / IWH / ZEW, Fortschritte beim Aufbau Ost. Fortschrittsbericht wirtschaftswissenschaftlicher Institute über die wirtschaftliche Entwicklung in Ostdeutschland, 2002

Entretien avec le Prince Alexandre de Saxe, chargé de mission auprès du Ministre-Président du Land de Saxe, en charge des relations internationales (avril 2006)

HyPOVEREINSBANK, Fit für die Zukunft? Die Bundesländer im Vergleich, Hambourg, 2007

IW Consult, Viertes Bundesländer-Ranking, étude réalisée par IW Consult, en partenariat avec l'Initiative Neue Soziale Marktwirtschaft et l'hebdomadaire Wirtschaftswoch, Cologne, septembre 2006, (www.insm.de) « Perspektiven Ostdeutschlands 15 Jahre danach », DB Research, n 306, novembre 2004

PROGNOS AG, Zukunftsatlas 2007, étude publiée en coopération avec le quotidien Handelsblatt, mars 2007 RIEDEL J., «Technologie- und Innovationspolitik in Sachsen », WSI, Diskussionspapier n 106, septembre 2002 SACHVERSTÄNDIGENRAT ZUR BEGUTACHTUNG DER GESAMTWIRTSCHAFTLICHEN ENTWICLUNG, Jahresgutachten 2004/05, novembre 2004

Standort Sachsen im Vergleich mit anderen Regionen (2005), Freistaat Sachsen, Staatsministerium für Wirtschaft und Arbeit (www.sachsen.swma.de)

Wirtschaftswoche, dossier sur la Saxe, $\mathrm{n}^{\circ}$ 19/2006

www.sachsen.de ; www.wfs.de ; www.fraunhofer.de. 


\title{
Brandebourg : la quête d'une nouvelle image
}

\author{
Marie-Hélène Pautrat
}

Land de tradition agricole et industrielle, le Brandebourg, recréé dans ses frontières actuelles le 3 octobre 1990, présente, dix-huit ans après la chute du Mur, une situation contrastée. Chômage de masse, désindustrialisation, désenchantement et départ des jeunes les plus qualifiés, mais aussi très forte hétérogénéité entre les régions du Land rendent compte de la complexité d'une situation économique et sociale précaire qui perdure. Alors que le processus de rattrapage stagne, les autorités entendent afficher le visage d'une région résolument tournée vers l'avenir. Le Brandebourg espère tirer profit de sa situation géographique au centre d'une Union européenne à 27 et affirme haut et fort, contre toute fatalité, ses atouts en termes d'implantation économique. C'est dans ce contexte que les autorités tentent d'enclencher une dynamique de croissance s'appuyant à la fois sur des noyaux de croissance et des infrastructures scientifiques et d'innovation prometteuses, en particulier dans les sciences de la vie. *

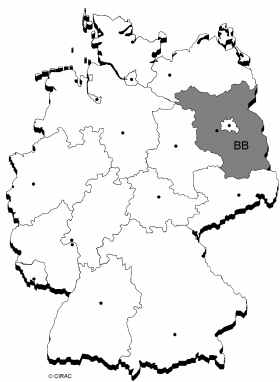

Le Brandebourg, $5^{\mathrm{e}}$ Land en termes de superficie $\left(29479 \mathrm{~km}^{2}\right)$, dispose d'une frontière commune de $252 \mathrm{~km}$ avec la Pologne. Sa particularité est qu'il entoure entièrement la capitale fédérale et se trouve de ce fait privé de centre géographique. Peu peuplé (2,6 millions d'habitants), il se caractérise aussi par une très faible densité de population : $87 \mathrm{hab} / \mathrm{km}^{2}$ seulement (Berlin : $3807 \mathrm{hab} / \mathrm{km}^{2}$ ). La population se concentre dans les principales villes dont Potsdam (capitale, 148691 hab.), Cottbus (105 309), Francfort sur Oder (63 748) ou Brandebourg sur la Havel (73 783), et à la périphérie de Berlin.

Brandebourg/Berlin : une région métropole à la recherche d'un équilibre territorial

Le Brandebourg est le seul Land oriental à avoir vu sa population augmenter légèrement dans l'ensemble depuis 1991 : mais il s'agit pour l'essentiel des conséquences de l'étalement urbain autour de Berlin (suburbanisation) et de la pression migratoire sur les zones de banlieue. Depuis 2001, le mouvement s'inverse d'ailleurs, de sorte que le Brandebourg pourrait perdre $7 \%$ de sa population d'ici 2020 du fait du vieillissement démographique.

Dès lors, on ne peut aujourd'hui penser le Land de Brandebourg sans l'associer à Berlin, Land et capitale fédérale. Tous deux constituent ensemble une entité de près de 6 millions d'habitants à l'échelle européenne. Mais la région Berlin/Brandebourg n'en présente pas moins de très fortes disparités : $80 \%$ de ses habitants, soit 4,5 millions, résident dans la zone de concentration urbaine de Berlin et sa zone d'influence élargie. L'essor du Brandebourg

\footnotetext{
*Version actualisée d'un article paru dans Regards sur l'économie allemande, n 68, octobre 2004.
} 
semble plus que jamais passer par des synergies avec Berlin qui, en exploitant les complémentarités des deux Länder, peuvent leur permettre d'accéder conjointement au rang de région majeure en Europe. C'est le sens de la coopération renforcée, concrétisée à partir d'avril 1995 par la signature d'un traité inter-étatique entre ces deux Länder et déclinée depuis par différents outils : elle leur permet de planifier et de réaliser en commun l'aménagement durable de leur territoire respectif.

Berlin au centre du Brandebourg

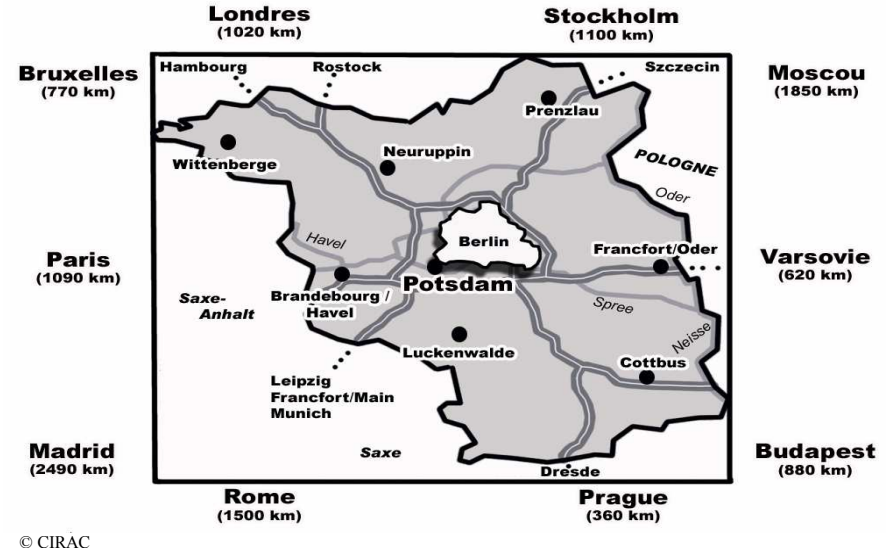

Dans les faits pourtant, la séparation politique a été entérinée avec l'échec de la fusion à l'issue du referendum de 1996. A défaut de perspectives tangibles de fusion dans les prochaines années (un nouveau referendum est néanmoins évoqué dans les milieux politiques à partir de 2009), les autorités des deux Länder s'efforcent depuis de multiplier les actions de coordination, notamment dans le domaine économique, et les exemples pratiques de coopération : représentation commune à Bruxelles, fusion envisagée en 2008 des agences de promotion économique des deux Länder, actions communes dans le domaine du commerce extérieur, coopération renforcée dans le cadre de la tâche commune Verbesserung der regionalen Wirtschaftsstruktur («amélioration de la structure économique régionale ») afin d'appréhender les conséquences probables de la nouvelle programmation financière 2007-2013 pour les fonds structurels européens. Celle-ci prévoit en effet des niveaux d'aides différents entre Berlin et les régions brandebourgeoises limitrophes, susceptibles de fausser les politiques de développement économique. Enfin, les deux Länder se présentent désormais ensemble dans toutes leurs actions de promotion internationale comme un espace économique et de vie commun sous l'étiquette de Hauptstadtregion Berlin/Brandenburg : « région-capitale ».

\section{Un rattrapage économique inachevé}

Avec un PIB de 49,5 milliards $€$ en 2006, l'économie brandebourgeoise pèse toujours peu $\left(2,14 \%\right.$ ) dans le PIB allemand (2 307 milliards $€$ ) : elle arrive en $12^{\mathrm{e}}$ position, juste derrière la Saxe-Anhalt. Alors que sa dynamique de croissance est restée supérieure entre 1995 et 2001 à la moyenne des Länder de l'ouest à faible croissance, la machine s'est grippée en 
2002 dans le triple contexte d'une conjoncture déprimée, de transformations structurelles inachevées dans le bâtiment et l'artisanat, et des difficultés du commerce de détail. Sousreprésentation des branches tirant la croissance allemande, tissu industriel composé à plus de $97 \%$ de très petites entreprises insuffisamment orientées à l'export : le Brandebourg, plus encore que les autres länder orientaux, peine à tirer profit de la dynamique économique allemande actuelle. Cette situation accentue la stagnation du phénomène de convergence observée depuis 1997. Doublée d'un ralentissement des recettes fiscales, elle pèse sur la situation financière du Land qui peine à maintenir ses dépenses d'infrastructures.

En prix courants, le PIB par habitant atteignait $19386 €$ en 2006, en deçà de la moyenne des nouveaux Länder (moyenne RFA en 2005: près de $28000 €$ ). Mais dans le même temps, le PIB par actif s'élevait à $48853 €$ en 2006, au deuxième rang des Länder orientaux ; le Brandebourg est désormais distancé par la Saxe-Anhalt. La productivité atteint en moyenne $74 \%$ du niveau national. Le retard peine donc à se combler, même si les statistiques masquent une situation inégale selon les secteurs d'activité et les entreprises, et que la structure par taille des entreprises industrielles des nouveaux Länder joue pour beaucoup dans cet effet statistique.

La productivité dépasse ainsi le niveau ouest-allemand dans certaines branches comme la chimie, l'industrie du bois, et s'avère largement supérieure, dans certaines filiales d'entreprises étrangères ou ouest-allemandes, à celle d'entreprises comparables à l'ouest (jusqu'à $140 \%$; source : ZAB). Elle reste néanmoins de beaucoup supérieure à celle enregistrée chez ses voisins et compétiteurs (République tchèque, Hongrie, Pologne).

Le marché du travail demeure certainement le principal défi pour la coalition SPD/CDU au pouvoir. En janvier 2007 en effet, 220903 chômeurs ont été enregistrés. Le taux de chômage se maintient à un niveau très élevé malgré une amélioration statistique continue depuis 2005 (16,6 \% en janvier 2007), due à une conjoncture plus favorable, alors qu'il demeurait supérieur à $18 \%$ depuis 1997. Mais cette embellie statistique ignore certains districts périphériques, trop éloignés du pôle Berlin/Potsdam pour bénéficier d'un marché du travail plus dynamique attirant des travailleurs migrants, dont le taux de chômage continue de flirter avec la barre des $20 \%$ (Eberswalde : 19,4 \% ; Cottbus : 20 \%). Dans le même temps, le nombre d'actifs décroît de manière quasi continue depuis 1995 pour atteindre 1,005 million en 2005 : il se situe aujourd'hui très au-dessous du 1,270 million enregistré après l'unification (1991).

En dépit de salaires toujours inférieurs au niveau ouest-allemand, d'une faible adhésion des entreprises brandebourgeoises aux accords de branche (moins de $30 \%$ ) et d'un marché du travail flexible dans les faits, le problème est donc toujours la capacité de créer de l'emploi nouveau et de réduire le déficit structurel d'emplois réguliers. Le Brandebourg ne parvient pas à résorber le socle de chômeurs de longue durée. Cette situation sociale difficile constitue d'ailleurs le terreau de l'implantation du parti PDS/Die Linke, qui détient 29 des 88 sièges au parlement régional depuis les élections de 2004, après avoir capitalisé les suffrages des chômeurs et d'un électorat socialement fragilisé.

Dix-sept ans après l'unification, le Brandebourg, terre des anciens grands domaines fonciers, a perdu son caractère agricole, conséquence de profondes restructurations. Aujourd'hui, avec 3,7 \% des actifs occupés, le secteur agricole (1,9\% du PIB) ne pèse proportionnellement guère plus que dans les autres Länder, à l'exception notable du Mecklembourg (2,9\%). La structure économique du Land est dominée par les services (73,2 \% en 2005), la part de l'industrie n'étant que de $24,9 \%$. 
Le Brandebourg peut se prévaloir d'une tradition industrielle qui s'est maintenue du temps de la RDA. Or avec les régions rurales, ce sont précisément les anciens fiefs et secteurs industriels traditionnels (construction automobile, chimie, construction ferroviaire et aéronautique) qui ont été le plus frappés au cours des années 1990 par les restructurations. Aujourd'hui, la base industrielle du Brandebourg (hormis le bâtiment, secteur encore en surcapacités), certes assainie et stabilisée, demeure inférieure en termes d'emplois et de valeur ajoutée brute à celle des Länder occidentaux, alors que le secteur des services est comparable.

Le profil industriel du Brandebourg est désormais marqué par quelques secteurs prépondérants comme la construction automobile (DaimlerChrysler à Ludwigsfelde, mais aussi ZF Getriebe GmbH Werk Brandenburg, Goodyear Dunlop Tires Germany à Fürstenwalde), l'industrie alimentaire (10 400 salariés, 147 entreprises ; CA : 2,5 milliards $€$ en 2005), l'industrie des métaux (13 900 salariés, 178 entreprises ; CA : 2,6 milliards $€$ à Brandebourg/Havel, Hennigsdorf ou encore Eisenhüttenstadt, créée de toutes pièces dans les années 1960 autour du complexe sidérurgique alimentant l'industrie de la RDA), ou l'industrie chimique. Avec 5229 salariés et un chiffre d'affaires de 1,2 milliard $€$ en 2005, la chimie est concentrée autour de quelques pôles principaux (Schwarzheide, également Premnitz, Oranienburg, Fürstenwalde ou Guben) et portée par des entreprises phares comme BASF qui a installé son troisième plus important site de production mondial à Schwarzheide. Autour de ces secteurs se développent des réseaux de sous-traitance et de services.

L'industrie manufacturière, malgré une croissance ralentie, restait ainsi en 2005 un pilier du développement économique. En 2005, ce sont les industries du papier, de la chimie, du bois, de la production et de la transformation des métaux, du caoutchouc et de la plasturgie qui ont ainsi enregistré le plus fort chiffre d'affaires, tandis que le secteur de l'automobile, de la construction mécanique, du verre ou de l'extraction reculaient.

Structure industrielle du Land de Brandebourg (industrie manufacturière - 2005)

\begin{tabular}{|lcc|}
\hline Principales branches & Chiffre d'affaires $(\boldsymbol{e n ~ m i o} \boldsymbol{\epsilon})$ & Salariés \\
Industrie alimentaire & 2492,6 & 10400 \\
Textile/habillement & 74,8 & 743 \\
Bois & 1123,5 & 3227 \\
Papier/Imprimerie & 1262,5 & 5640 \\
Chimie/Caoutchouc/Plasturgie & 2450,7 & 9179 \\
Verre/Céramique & 926,5 & 5011 \\
Production/transformation des & 1642,8 & 13939 \\
métaux & 829,1 & 6348 \\
Construction mécanique & 2222,3 & 11869 \\
Construction automobile & $(234)^{*}$ & 1459 \\
Technologies IC & & \\
Techniques médicales/Mesures et & 305,3 & 2959 \\
asservissement/optique & & \\
\hline
\end{tabular}

Source : Jahreswitschaftsbericht 2006. *) Données 2004

Outre la restructuration des noyaux industriels, les années 1990 ont permis la mise à niveau des infrastructures et des réseaux de communication. L'effort a également porté sur l'essor d'un secteur des services (commerce, transports, information, services financiers, tourisme), qui occupe dorénavant plus des deux tiers des actifs du Land. Mais les résultats en termes de croissance et de créations d'emplois restent insuffisants, et les fondations d'une économie 
tirée par des branches à forte intensité en R\&D et des services modernes restent fragiles, notamment face aux exigences liées à la globalisation des activités économiques. La structure économique du Brandebourg, caractérisée par la prédominance de petites et moyennes entreprises aux capacités financières trop précaires pour réaliser les investissements nécessaires, en particulier dans le domaine de la $R \& D$, reste un handicap. Surtout, les disparités régionales demeurent fortes au sein du Land.

Au regard d'un bilan économique très contrasté dix-sept ans après la chute du Mur, le Brandebourg tente à son tour d'impulser une nouvelle dynamique à son économie. Dès lors, la discussion a porté ces dernières années sur l'utilisation optimale des aides, entre tenants d'une concentration des efforts sur les régions périphériques et rurales les plus nécessiteuses, ou d'une réorientation sur la ceinture porteuse de croissance autour de Berlin.

\section{Le choix d'un renforcement des noyaux de croissance}

A la mi-2004, les grandes lignes de la politique économique du Land ont été redéfinies (dans l'esprit du $2^{\mathrm{e}}$ Pacte de solidarité 2005-2019) et se focalisent dorénavant sur trois piliers principaux : un soutien spécifique aux entreprises du Mittelstand (aides à la création et à l'implantation d'entreprises, soutien direct aux investissements) ; un recentrage des aides économiques sur les secteurs économiques porteurs ; une redéfinition de la politique d'aides à l'innovation (Landesinnovationskonzept 2006).

Au plan sectoriel, cette stratégie se traduit par la constitution de pôles autour de quelques branches à fort potentiel de croissance et d'innovation et par la concentration des aides sur quelques secteurs technologiques spécifiques (Branchenkompetenzfelder) : sciences de la vie/ biotechnologies, aéronautique, équipement automobile, technologies ferroviaires, médias/ information et communication, services/logistique, chimie/plasturgie/secteur pétrolier, énergie/ technologies énergétiques, agroalimentaire, production et transformation des métaux/mécatronique, optique, géoinformation, industrie du bois, industrie du papier et tourisme. Entreprises innovantes et organismes de recherche sont appelés à coopérer dans le cadre de réseaux, comme par exemple dans le secteur des microtechnologies et de la microélectronique à Francfort/Oder ou, au Sud de Berlin, de l'aéronautique : le pôle Berlin-Brandenburg Aerospace Allianz entend faire de la région le troisième site allemand de ce secteur.

Globalement, ces mesures de concentration des aides sur quelques «noyaux de croissance » bénéficient aux zones urbaines à fort potentiel de croissance économique ou scientifique, conformément à la stratégie du gouvernement du Land : «renforcer les points forts » (Stärken stärken). Géographiquement, quelques districts (Teltow-Fläming, Oberhavel, Dahme-Spreewald) et agglomérations (Brandebourg/Havel, Cottbus) parviennent à tirer les bénéfices d'une dynamique industrielle ou de services.

La capitale du Land, Potsdam, est ainsi devenue un centre réputé pour les activités de la high-tech allemande, qui se structure autour de l'industrie cinématographique et audiovisuelle (Potsdam est le site historique des studios de Babelsberg) : école supérieure du cinéma et de la télévision, High-Tech Center, studios d'enregistrement, entreprises performantes (production de DVD, films en 3D). A quelques exceptions près (Oberspreewald-Lausitz, par exemple), les régions jouxtant la capitale Berlin enregistrent le meilleur développement économique.

Par ailleurs, les mesures décidées dans le cadre de la politique de coopération Bund/ Länder (tâche commune « amélioration de la structure économique régionale ») tout comme 
les nouvelles orientations pour l'utilisation des fonds structurels européens définies dans l'objectif de renforcer la Stratégie de Lisbonne viennent conforter cette politique de concentration sur les points forts du Land.

\section{Le défi de l'élargissement}

Mais l'avenir de l'économie brandebourgeoise dépend aujourd'hui fortement de sa position centrale dans une Europe élargie, avec ses atouts et ses contraintes. Situé avant 2004 aux confins de l'UE 15 et des zones économiques les plus actives, le Brandebourg bénéficie aujourd'hui du déplacement du centre de gravité européen. Il s'agit de tirer profit de la position géographique du Land, centre de trafic et pont vers l'Europe orientale, grâce à des infrastructures de transport - ferroviaires, routières, fluviales - et des liaisons internationales (notamment l'axe autoroutier est-ouest A2) rénovées. Par ailleurs, le chantier du futur aéroport Berlin Brandenburg International, longtemps contesté, vient de débuter à l'automne 2006 pour pouvoir concentrer le trafic aérien et donner à la région les moyens de ses ambitions économiques. Dès 2011, il devrait pouvoir accueillir entre 22 et 25 millions de passagers par an.

Pourtant, une partie des effets de l'intégration s'est progressivement fait sentir dès avant l'entrée dans l'UE des pays d'Europe centrale et orientale. Le Brandebourg s'est préparé 10 années durant à cette échéance par une intensification de la coopération transfrontalière avec les voïvodies polonaises de Lubusz et de Poméranie occidentale : actions dans le cadre des programmes communautaires Interreg (soutien aux régions frontalières, membres de 3 Eurorégions), développement concerté de la région de l'Oder, programmes bilatéraux dans les domaines de la formation, de la lutte contre la criminalité, de l'environnement, instauration d'un dialogue entre partenaires sociaux, contacts entre partenaires économiques, coopération institutionnelle, programme du ministère de l'économie pour favoriser les investissements, etc.

L'élargissement a par ailleurs apporté des perspectives nouvelles en matière d'échanges extérieurs, clefs de la dynamique économique de la RFA et possible moteur de croissance pour le Land. Alors qu'en 2005, les exportations des entreprises brandebourgeoises s'élevaient à 6,55 milliards $€$ (contre 5,46 milliards $€$ en 2004), les seuls échanges vers les pays de l'UE se montaient à 4,57 milliards $€$ (contre 3,58 milliards $€$ en 2004). La Pologne représentait en 2005 le premier client des exportateurs brandebourgeois (858 millions $€$ ), suivie des Pays-Bas, des Etats-Unis, de l'Italie puis de la France. Quant à la Russie, si une part significative des exportations du Land s'y dirige (298 millions $€$ ), elle est surtout le premier importateur (pétrole et gaz naturel) avec un cumul de 3,81 milliards $€$ (sur un total d'importations de 8,46 milliards $€$ en 2005).

Les Länder est-allemands peuvent en effet faire valoir leur connaissance particulière des pays d'Europe centrale et orientale. Dans le même temps, les nouveaux membres bénéficient de la politique de cohésion européenne via la nouvelle programmation des fonds structurels qui soutiendront leur économie (voisine du Brandebourg, la Pologne a reçu 12,8 milliards $€$ entre 2004 et 2006 et peut espérer jusqu'à 63 milliards $€$ pour la période 2007-2013). Mais la faible densité industrielle, la prépondérance d'entreprises de petite taille en proie à des difficultés de financement, le manque de personnel qualifié dans certains secteurs, notamment en raison du différentiel des salaires, et des investissements notoirement insuffisants en R\&D sont autant de facteurs qui pénalisent sur les marchés extérieurs les entreprises est-allemandes par rapport à leurs homologues d'Allemagne occidentale. Et l'atout de la proximité 
géographique avec les nouveaux marchés d'Europe centrale, particulièrement pertinent pour le Brandebourg, risque de pâtir du ralentissement du développement des liaisons routières et ferroviaires, du ressort de communes et Länder sous contrainte budgétaire.

Pourtant, et en dépit des mesures transitoires (notamment la limitation de la libre circulation des travailleurs), l'élargissement inquiète : le taux de participation aux dernières élections européennes de 2004 s'est ainsi révélé extrêmement bas dans le Brandebourg (moins de $27 \%$ ). L'ouverture à l'Est renforce les craintes sur l'attractivité du Land, mise à mal ces dernières années par une série d'enquêtes comparatives sur la compétitivité des Länder (voir, par exemple, l'étude Cap Gemini). Dans un contexte de sous-emploi, c'est la question des coûts salariaux, avantage compétitif des nouveaux Etats membres, qui prédomine. Elle trouve par ailleurs son prolongement dans le débat sur une zone à bas niveau de salaires à l'Est, que récusent les autorités du Land qui refusent de s'engager dans une concurrence avec les pays d'Europe centrale et orientale sur l'emploi et les coûts salariaux.

L'agence de développement économique ZukunftsAgentur Brandenburg (ZAB), créée en 2001, s'emploie plutôt à établir un inventaire des atouts du Brandebourg comme site de production compétitif (308 entreprises étrangères s'y sont implantées) et alternative possible aux délocalisations d'entreprises allemandes: qualité d'infrastructures modernes et rénovées, surfaces d'activités disponibles et à des prix $/ \mathrm{m}^{2}$ limités, programmes multiples d'aide aux investisseurs (aides à l'innovation, à la formation, mesures fiscales, crédits à taux privilégié), proximité d'un grand marché (Berlin, marchés tchèque et polonais).

\section{Formation, recherche et innovation : un potentiel à valoriser}

Avant tout, le Brandebourg entend miser sur le niveau de qualification et de formation élevé de sa main d'œuvre et d'un environnement scientifique de qualité, résultat d'un processus mené durant les quinze années suivant l'unification. Le Land compte aujourd'hui 40000 étudiants dans ses 4 universités (Université de Postdam, niversité européenne Viadrina de Francfort sur Oder, Université technique de Cottbus, Université sur les médias de PostdamBabelsberg) et 5 Fachhochschulen (Potsdam, Brandenburg, Wildau, Eberswalde, Lausitz). Avec plus de 20000 étudiants et 3700 emplois scientifiques, Potsdam figure en tête des villes allemandes pour la part de l'emploi scientifique dans la population. A une plus large échelle, ces établissements viennent gonfler le vivier des 130000 étudiants berlinois : ils constituent ensemble un dispositif de formation d'une main d'œuvre hautement qualifiée, même si, il est vrai, les établissements ont subi entre 1999 et 2004 des restrictions de financement drastiques de la part de leur tutelle respective, et notamment du Land de Berlin.

A cette offre de formation s'ajoute le potentiel scientifique : instituts Max-Planck, instituts Fraunhofer, instituts WGL et centres Helmholtz, établissements de recherche du Bund, des centres de recherche réputés comme l'institut de recherche sur le climat (Potsdam) ou le centre de recherche sur les géosciences (Potsdam). La recherche publique joue un rôle essentiel dans le cas du Brandebourg, comme dans les autres Länder orientaux, où il s'agit de pallier l'insuffisance de l'activité de R\&D d'entreprises souvent trop petites. Par ailleurs, 20 centres de technologies facilitent la création d'entreprises technologiques en entretenant le contact avec les organismes de recherche et en proposant des services adaptés.

La réorientation de la politique économique du Land tend dès lors à focaliser ses efforts sur l'investissement dans la R\&D et les politiques d'innovation. Une palette d'instruments a 
été proposée dans le cadre de la stratégie Landesinnovationskonzept Brandenburg 2006 (LIK) adoptée en 2006: aides à la création d'entreprises innovantes et à l'implantation d'entreprises high-tech, transfert de technologie, définition d'objectifs communs BerlinBrandebourg, focalisation sur quelques secteurs-clés, réorientation des fonds FEDER. L'exemple du secteur des sciences de la vie, qui est le secteur au plus fort potentiel, fait de l'espace Berlin-Brandebourg un noyau de la recherche allemande à visibilité internationale (particulièrement représentés : le génie génétique, la bioinformatique, les biotechnologies vertes, l'immunologie, la cardiologie).

L'atout primordial de la "région-capitale » est qu'elle bénéficie d'un tissu scientifique très dense, d'hôpitaux universitaires renommés (la Charité à Berlin est le plus grand CHU d'Europe) et de 6 parcs biotechnologiques. Trois d'entre eux se situent dans le Brandebourg qui peut faire valoir, par rapport à Berlin, l'atout de surfaces disponibles répondant aux normes d'implantation des laboratoires de pointe, à des coûts comparativement moins élevés. Cette densité unique renforce la capacité de la région à constituer des réseaux entre organismes de recherche, partenaires économiques, universités et entreprises.

Dans le secteur des biotechnologies par exemple, la Biotechnologie-Region BerlinBrandenburg (170 entreprises, les deux tiers dans la capitale allemande, 3200 emplois), issue de la participation commune des deux Länder au concours fédéral Bio-Regio (1996), s'impose aujourd'hui comme le principal pôle allemand de R\&D dans les biotechnologies, notamment pour la biomédecine, les techniques médicales, la bioinformatique et les biotechnologies des plantes. La mise en réseau de la recherche pour accélérer les transferts de technologie (plus d'une douzaine de réseaux recensés dans la région pour ce seul secteur), l'aide à la création d'entreprise et au financement y sont coordonnées par l'agence BioTOP, commune aux Länder de Berlin et du Brandebourg et cofinancée par des entreprises privées des secteurs de la chimie et de la banque. Reste maintenant au Land à conforter le potentiel de croissance de la branche pour répondre à l'objectif fixé de devenir un des principaux clusters européens et mondiaux dans les sciences de la vie.

APRÈs la phase douloureuse des RestruCturations et des ajustements, partiellement inachevée, les autorités brandebourgeoises s'appliquent à transmettre l'image d'une région tournée vers l'avenir. Si le Brandebourg n'avait jusqu'à présent pas été aidé par sa localisation géographique à la périphérie de l'Europe, la situation peut évoluer dans une Europe à 27, et les autorités s'emploient à vanter ses atouts : atouts d'une économie qui poursuit sa transition avec des niveaux de salaires, de prix et de loyers modérés, et présente dans le même temps des infrastructures modernes et un haut niveau de qualification. Et le Land continue de promouvoir les synergies avec la capitale Berlin, même si la fusion politique est dorénavant jugée improbable à moyen terme. Malgré cela, le tissu industriel reste insuffisamment dense pour garantir une croissance durable. Partant de ce constat, les pouvoirs publics s'emploient à concentrer leurs efforts sur quelques pôles d'activité à fort potentiel. La dynamisation du Brandebourg passe également par la valorisation du potentiel scientifique et le passage à une économie basée sur la connaissance et l'innovation. C'est ainsi que le Brandebourg espère rompre la spirale vicieuse qui plane sur les Länder orientaux et créer sur place les conditions d'emploi d'une main d'œuvre qualifiée. 


\section{Indications bibliographiques}

CAP GEMINI, ERNST \& Young, Studie : Standortattraktivität. Die Unternehmerfreundlichkeit der Bundesländer im Vergleich. Kurzfassung, septembre 2002

LAND BRANDENBURG/MinisteriUm FÜR WirTSCHAFT, Jahreswirtschaftsbericht 2006, Potsdam (www. wirtschaft.brandenburg.de)

LANDESBETRIeB FÜr DATENVERARBeitung UND STATISTIK LAND BRANDENBURG, Statistisches Jahrbuch 2006, Potsdam, 2006

Life Sciences in der Hauptstadtregion Berlin-Brandenburg, Berlin, Berlin Partner GmbH, décembre 2006 (www.zwb-brandenburg.de)

Pautrat M.-H., «Pologne-RFA : les relations s'intensifient », Regards sur l'économie allemande, $\mathrm{n}^{\circ} 62$, juillet 2003

Statistik Berlin-BrandenBurg, Statistischer Bericht A VI- m 03/07. Arbeitsmarkt im Land Brandenburg, mars 2007

ZuKunfTSAgENTUR BRANDENBuRg, Der Standort Brandenburg : Eine Alternative zur Auslandsverlagerung - insbesondere für höherwertige Investitionen, 4e éd., janvier 2006 (www.zab-brandenburg.de)

StAATSKANZLEI BeRlin / STAATSKANZlei BRANDENBURG, Fortschrittsbericht über die Zusammenarbeit zwischen den Ländern Brandenburg und Berlin sowie die weitere Zusammenlegung von Behörden und Sonderbehörden, (http://www.stk.brandenburg.de/media/lbm1.a.4856.de/bb_fortschrittsbericht2006.pdf), Berlin, Potsdam, 21 novembre 2006 



\section{Index des cartes, tableaux et encadrés}

\section{CARTES ET IMAGES}

- Concentration des activités dans l'espace européen :

l'Allemagne est au cœur de la « banane bleue »...

- Emploi dans l'industrie manufacturière de haute

et moyenne-haute technologieen Europe ...

.

- Répartition territoriale des secteurs à forte croissance en Allemagne ...................................................... 13

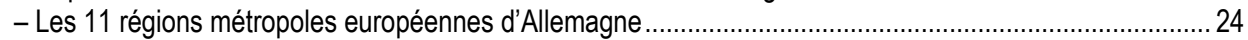

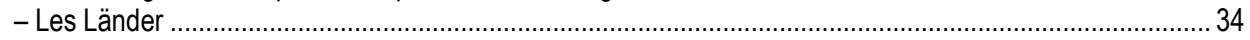

- Concentration régionale des secteurs-phares de l'industrie allemande .................................................... 37

- Régions allemandes : dynamique comparée et forces actuelles............................................................... 49

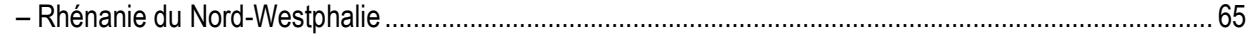

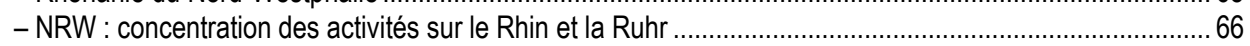

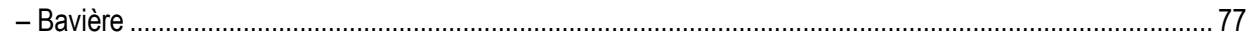

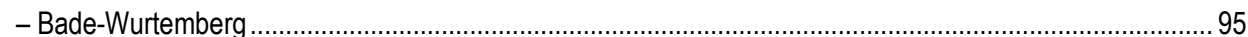

- Bade-Wurtemberg : 4 districts administratifs et un réseau de $12 \mathrm{CCl}$................................................. 100

- Benchmarking régional des pôles de compétitivité allemands en termes de potentiel............................. 101

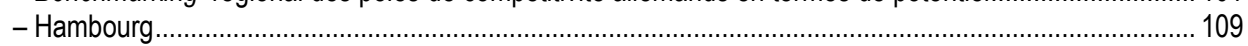

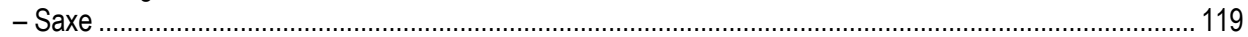

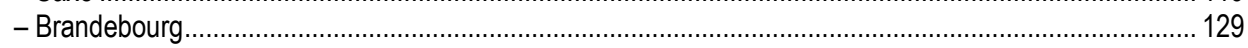

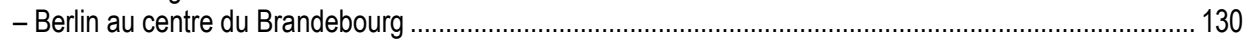

\section{TABLEAUX}

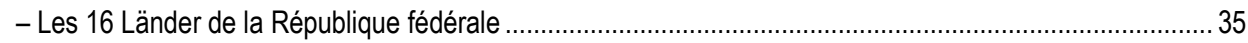

- Structure de l'économie des Länder (2005) - part respective des secteurs............................................. 36

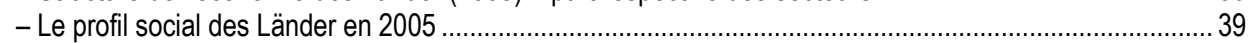

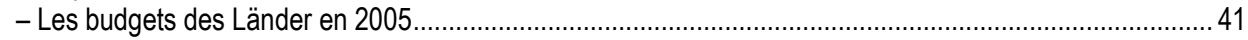

- Capacité financière des Länder avant/après le Länderfinanzausgleich - 2005 .......................................... 42

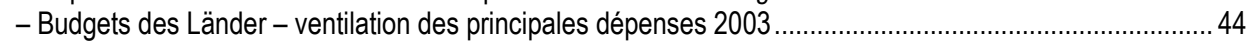

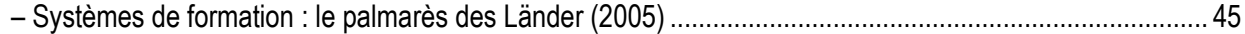

- Indicateurs économiques et sociaux de l'Allemagne de l'est - 1991-2006 ..............................................52

- Aspects financiers et économiques des nouveaux Länder en 2003 .......................................................57

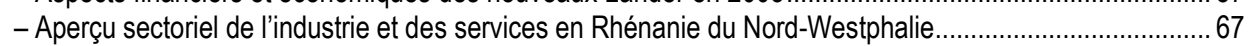

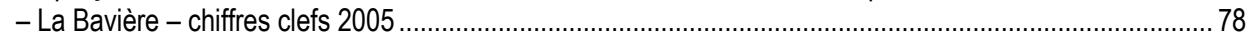

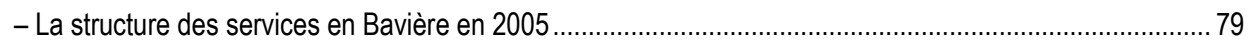

- Diversité de l'industrie bavaroise et son apport à l'industrie allemande - quelques exemples (2005)........ 82

- Production d'électricité par les compagnies publiques bavaroises 1955-2003 ........................................... 89

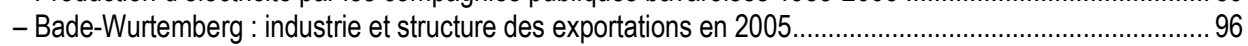

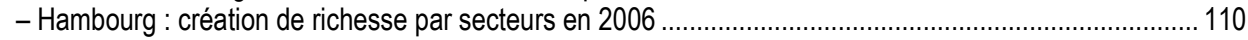

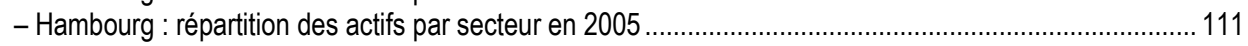

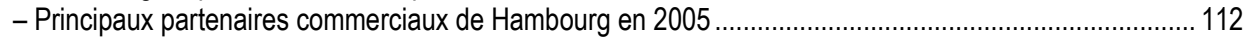

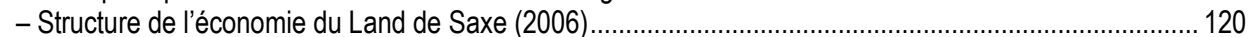

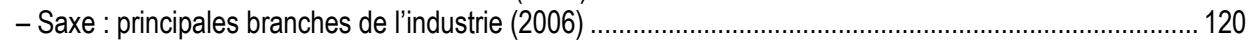

- Structure industrielle du Land de Brandebourg (industrie manufacturière - 2005) ................................. 132 


\section{ENCADRÉS}

- Le principe de subsidiarité au cœur du libéralisme organisé 15

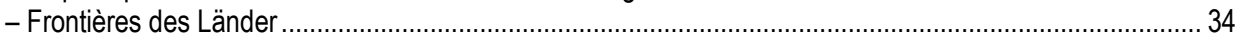

- Les Länder est-allemands n'ont plus rien de «l'ex-RDA » ..................................................................... 38

- Solidarpakt l et Solidarpakt II ................................................................................... 56

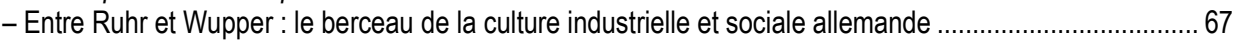

- Un exemple de mise en réseau des compétences dans le design : Zeche Zollverein ................................ 72

- Quand le football entretient lien social et culture de la compétitivité ........................................................... 75

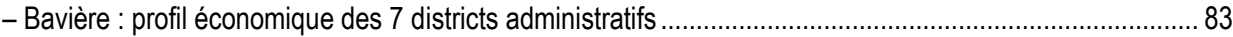

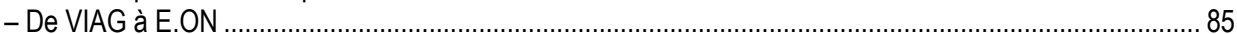

- Bavière : Le dispositif de l'ex-Offensive High-Tech au cœur de l'actuelle Cluster-Offensive....................... 92

- Un exemple de fonctionnement en partenariat : Hollister's MotorCycles ................................................. 98

- Le dispositif de transfert et de recherche appliquée du Bade-Wurtemberg.............................................. 105

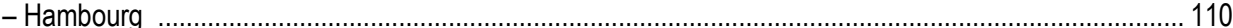

- POWER 8 préserve le site de production de Hambourg …………........................................................ 114

- Hambourg : la Hafencity, emblème de l'expansion de la ville ................................................................ 115

- Saxe : le renouveau de l'industrie automobile ....................................................................................... 121

REMERCIEMENTS POUR L'AIMABLE AUTORISATION DE REPRODUCTION DES CARTES ET IMAGES :

- à Deutsches Zentrum für Luft- und Raumfahrt (DLR) pour l'image satellite :

Concentration des activités dans l'espace européen : l'Allemagne est au cœur de la «banane bleue » (p.11)

- à Eurostat, Eurographics (C) Communautés européennes pour la carte :

Emploi dans l'industrie manufacturière de haute et moyenne-haute technologie en Europe (p. 12)

- à Prognos AG pour les cartes :

Répartition territoriale des secteurs à forte croissance en Allemagne (p. 13)

Régions allemandes : dynamique comparée et forces actuelles (p. 49)

Benchmarking régional des pôles de compétitivité allemands en termes de potentiel (p. 101)

- à Bundesamt für Bauwesen und Raumordnung pour la carte :

Les 11 régions métropoles européennes d'Allemagne (p. 24)

- à Baden-Württembergischer Industrie- und Handelskammertag pour la carte :

Bade-Wurtemberg : 4 districts administratifs et un réseau de $12 \mathrm{CCl}$ (p. 100). 


\section{Les auteurs}

\section{Isabelle BOURGEOIS}

Maître de conférences, Université de Cergy-Pontoise,

Chargée de recherches au CIRAC,

Rédactrice en chef de Regards sur l'économie allemande

\section{Markus GABEL}

Docteur en sciences économiques,

Chercheur associé au CIRAC

\section{Marie-Hélène PAUTRAT}

Ingénieur d'études au CIRAC jusqu'en 2006

\section{Michèle Weinachter}

Maître de conférences, Université de Cergy-Pontoise,

Chercheur associé au CIRAC 


\section{OUVRAgES PARUS AUX ÉDITIONS DU CIRAC}

\section{- dans la collection Travaux et documents du CIRAC :}

Leo KIBler, René Lasserre, Marie-Hélène PaUtRat (dir.), Modernisation des services publics et management social en France et en Allemagne.

Consolidation des budgets publics, mise en concurrence et dérégulation des services d'intérêt général à l'échelle européenne, évolution des attentes des usagers-clients au regard de la flexibilité et de la qualité des services rendus, nécessité de promouvoir à l'échelon des Etats et des régions un environnement public stimulant pour l'activité économique... Ces changements imposent une rénovation des modèles classiques d'organisation de l'action publique et la mise en œuvre de nouvelles formes de management des ressources humaines. Cet ouvrage rassemble les contributions de chercheurs et responsables français et allemands portant sur la gestion de l'emploi et des compétences, la représentation du personnel et les relations sociales dans la sphère publique. Il en ressort un tableau différencié qui met en évidence les conditions et les modalités très variables selon lesquelles ces grands enjeux de la modernisation de l'Etat sont abordés de part et d'autre du Rhin.

Juillet 2007, 144 p., ISBN 978-2-905518-34-0 (20 €).

\section{Isabelle BOURGEOIs (dir.), Le modèle social allemand en mutation.}

Le modèle social allemand est en crise. Depuis 2003, gouvernement et partenaires sociaux ont commencé à le réformer : révision du système d'indemnisation chômage, revalorisation de l'apport individuel à l'assurancemaladie ou aux régimes de retraite, nouvelle approche globale de la politique salariale pour concilier compétitivité des entreprises et solidarité collective. Mais l'Allemagne doit aussi et surtout refonder son contrat social et trouver un nouvel équilibre entre le marché et la solidarité, entre la promotion de l'activité et la redistribution, entre les droits et devoirs respectifs de l'individu et de la collectivité.

Les réformes en cours impliquent aussi un débat sur les valeurs qui fonderont les nouveaux choix de société allemands et assureront le maintien de la performance économique du pays dans le cadre d'une globalisation assumée et d'un engagement européen réaffirmé. C'est dans la perspective d'une Europe compétitive dans l'économie du savoir que l'Allemagne inscrit la refondation de son modèle économique et social.

Septembre 2005, 208 p., ISBN 2-905518-33-2 (25€).

Hervé Joly (dir.), Formation des élites en France et en Allemagne.

Si de nombreux travaux récents abordent les élites en général, seules quelques initiatives isolées analysent, sur des aspects particuliers, les élites françaises actuelles. A l'inverse, en Allemagne, après une longue période d'occultation liée à des associations historiques embarrassantes, les publications sociologiques sur les élites foisonnent aujourd'hui de manière spectaculaire. Cette profusion traduit cependant plus une proclamation d'intérêt pour un concept qu'une restitution massive de recherches empiriques nouvelles.

Publier des textes d'auteurs français et allemands sur ce thème constitue donc une démarche inédite, développée en trois temps dans cet ouvrage : étude des conditions dans lesquelles se sont construites les différences observées dans la formation des élites des deux pays, présentation des enquêtes récentes sur le recrutement des élites politiques, administratives et économiques ; analyse de la manière dont, en pratique, les différences constatées se répercutent sur les conditions de travail en commun dans les diverses entreprises mixtes constituées depuis quelques années.

Juillet 2005, 228 p., ISBN 2-925518-32-4 (20 €).

Patricia Coмmun (dir.), L'ordolibéralisme allemand. Aux sources de l'économie sociale de marché.

L'ordolibéralisme a retrouvé une actualité scientifique. Il est considéré aujourd'hui comme un précurseur de l'institutionnalisme et, plus largement, d'un retour à une économie culturelle. L'ordolibéralisme, comme toute pensée économique, repose en effet sur des postulats philosophiques et s'inscrit dans un cadre éthique et culturel. C'est cette dimension philosophique et culturelle de l'ordolibéralisme allemand qui est analysée dans cet ouvrage collectif par des germanistes, des économistes et des philosophes français, anglais et allemands. Le livre aborde également la question de l'application politique de cette pensée économique, en apportant des 
éclairages sur certains des principes mis en œuvre, ainsi que sur l'action politique et idéologique entreprise par les ordolibéraux eux-mêmes en RFA dans les années d'après-guerre.

Juin 2003, 274 p., ISBN 2-905518-31-6 (32 €).

Isabelle BOURGEOIS (dir.), Allemagne 2001. Regards sur une économie en mutation.

Confronté au double défi de l'unification et de la mondialisation, le «modèle rhénan »semblait s'être englué dans ses pesanteurs et ses rigidités. Pourtant, le gouvernement Schröder est parvenu à sortir l'Allemagne de sa torpeur. Une nouvelle dynamique collective de modernisation a saisi pouvoirs publics, acteurs économiques, partenaires sociaux. Elle se fonde sur une acceptation raisonnée du changement. Mais le défi que relève la société allemande dépasse l'horizon mental national : c'est avant tout un choix européen assumé...

Juin 2001, 274 p., ISBN 2-905518-30-8 (32 €).

Jacques PATEAU, Une étrange alchimie. La dimension interculturelle dans la coopération francoallemande.

A travers des centaines d'interviews réalisées dans des entreprises appartenant aux secteurs les plus variés, l'auteur nous fait découvrir non seulement les différences dans les manières d'organiser le travail, de gérer ou de communiquer, mais surtout l'impact concret des développements historiques qui ont façonné nos cultures. Ce livre recèle des outils précieux pour comprendre la cohérence et l'intelligence de chaque système.

5 édition, novembre 2005, 254 p., ISBN 2-905518-29-4 (32 €).

Rémi LALLEMENT, L'unification sans miracle. L'économie allemande en mutation (1990-1995).

L'unification révèle les limites du «modèle allemand»: elle absorbe une bonne part des ressources nationales, exacerbe les conflits de répartition et, surtout, bat en brèche sa compétitivité internationale. En réponse à ces chocs, l'Allemagne s'est engagée dans un vaste aggiornamento en de nombreux domaines : des pratiques managériales aux conventions collectives, en passant par la politique industrielle. L'unification n'est pas seulement une épreuve de vérité, mais bel et bien l'occasion de renforcer les atouts de l'économie allemande en les adaptant aux exigences nouvelles de la compétition mondiale.

Octobre 1995, 176 p., ISBN 2-905518-28-6 (18,29€).

- dans la collection Deutsch-französische Studien zur Industriegesellschaft aux éditions Campus (Francfort/New York) :

Leo KIBLer, René Lasserre, Marie-Hélène Pautrat (dir.), Öffentlicher Dienst und Personalmanagement. Zur Verwaltungsreform in Deutschland und Frankreich. 2006, 212 p., ISBN 3-593-38178-8.

Anne-Marie Le GloanneC (ed.), Mehr oder weniger Staat ? Die Rolle des Staates in Deutschland nach 1989. 2003, 260 p., ISBN 3-593-37245-2.

Philippe D'IRIBARNE, Ehre, Vertrag, Konsens. Unternehmensmanagement und Nationalkulturen. 2003, 260 p., ISBN 3-593-37245-2.

\section{- autres publications du CIRAC :}

Regards sur l'économie allemande - Bulletin économique du CIRAC

5 numéros par an (abonnement : $200 €$ ). ISSN 1156-8992

CIRAC-Forum-Bulletin d'information pour la coopération franco-allemande dans les sciences humaines et sociales

4 numéros par an. Diffusé gratuitement par courrier électronique et consultable sur le site du CIRAC

Liste complète des publications du CIRAC sur le site : www.cirac.u-cergy.f 
Composition : CIRAC

CIRAC, c/o Université de Cergy-Pontoise

33 Boulevard du Port - 95011 CERGY-PONTOISE CEDEX

Impression : Dupli-print

2 rue Descartes - Z.I. Sezac - 95330 DOMONT

Dépôt légal : juillet 2007

Imprimé en France 


\title{
ALLEMAGNE : \\ COMPÉTITIVITÉ ET DYNAMIQUES TERRITORIALES
}

\author{
Sous la direction d'Isabelle Bourgeois
}

La compétitivité de l'économie allemande ne repose pas uniquement sur la puissance, la spécialisation sectorielle et la tradition exportatrice de son industrie, mais également sur la qualité de ses infrastructures et l'excellence d'une gestion territoriale fondée sur l'autonomie régionale et le polycentrisme. Dans le cadre du fédéralisme, c'est au niveau des Länder que, pour l'essentiel, se conçoit, se décide et se construit la dynamique des territoires et que s'affirme ainsi la capacité de l'Allemagne à faire face aux mutations structurelles d'une économie hautement développée et largement ouverte sur le monde.

La vitalité et la solidité de l'économie allemande sont dans une large mesure la résultante de dynamiques territoriales plurielles, dont cet ouvrage montre, à travers une série de portraits de Länder significatifs, qu'elles sont elles-mêmes les fruits de politiques de développement économique régionales inscrites dans la durée. Ainsi de la Bavière rurale devenue site hightech, du Bade-Wurtemberg qui s'est constitué en écosystème de l'excellence industrielle, de la Rhénanie du Nord-Westphalie, ancien 'pays noir' reconverti dans le multimédia et la logistique intermodale, de la Saxe qui, délivrée de l'économie planifiée de l'ex-RDA, n'a de cesse de redevenir un site industriel de premier plan au centre de la nouvelle Europe.

Pourtant, ce modèle de gestion territoriale polycentrique est lui-même confronté à l'épreuve du changement : la globalisation des activités et, plus encore, l'accélération de l'intégration européenne, placent aujourd'hui l'Allemagne face à un défi de gouvernance. Comment préserver l'autonomie des Länder, garante d'une concurrence régulée des intérêts régionaux au sein d'un fédéralisme coopératif, alors que «Bruxelles », au nom des impératifs de la compétitivité globale, tend à piloter les politiques structurelles jusqu’à l'échelon local ? Comment préserver institutionnellement, dans ce processus d'intégration communautaire aux logiques souvent contradictoires, cette diversité régionale qui fonde la prospérité et l'unité plurielles de l'Allemagne?

René Lasserre

Directeur du CIRAC

Professeur à l'Université de Cergy-Pontoise

$25 € \mathrm{TTC}$

ISBN : 978-2-905518-35-4

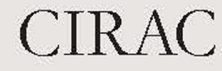

